ProQuest Number: 10131074

All rights reserved

INFORMATION TO ALL USERS

The quality of this reproduction is dependent upon the quality of the copy submitted.

In the unlikely event that the author did not send a complete manuscript and there are missing pages, these will be noted. Also, if material had to be removed, a note will indicate the deletion.

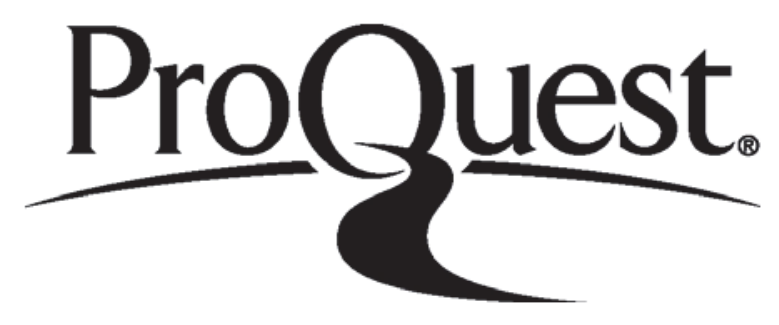

ProQuest 10131074

Published by ProQuest LLC (2017). Copyright of the Dissertation is held by the Author.

All rights reserved.

This work is protected against unauthorized copying under Title 17, United States Code Microform Edition (c) ProQuest LLC.

ProQuest LLC.

789 East Eisenhower Parkway

P.O. Box 1346

Ann Arbor, Ml $48106-1346$ 


\title{
Radio propagation in Frequency Selective Buildings
}

\author{
Marios Raspopoulos \\ Submitted for the Degree of \\ Doctor of Philosophy \\ from the \\ University of Surrey

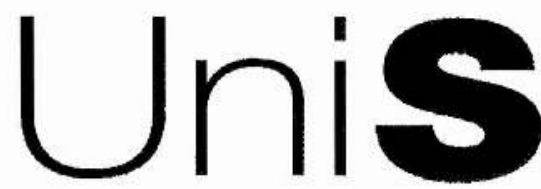 \\ Centre for Communication Systems Research \\ School of Electronics and Physical Sciences \\ University of Surrey \\ Guildford, Surrey GU2 7XH, UK
}

November 2007

(C) M. Raspopoulos 2007 


\section{Summary}

Predicting and improving Radio propagation conditions has been a major topic of research since wireless communications started to emerge. The aim is to increase signal coverage and reliability, meet the increased traffic demands and provide high quality signal to the higher levels of the OSI model. Moreover, the coexistence of various wireless networks in a wireless communication environment, operating on various frequency bands increases the need for a frequency selective solution for improving radio propagation conditions of the various networks. For this reason, this work proposes a novel way to improve and controllably manipulate radio propagation by transforming the building interfaces into frequency selective. Naturally, buildings can present some natural frequency selectivity. The web and void design of the individual blocks and their arrangement within a building wall/interface, creates a periodic structure, which exhibits frequency dependent transmission and reflection characteristics. This behaviour as well as the scattering behaviour of conventional periodic building structures have been studied through the RCWA method. However, since the internal structure and the parameters of the building interfaces are usually unknown, it is not currently very practical to utilise this natural frequency selectivity. This may change if an easy way is found to " $\mathrm{x}$-ray" the wall. Therefore, the novel way proposed, is to artificially transform the building interfaces into frequency selective ones, tuneable at a desired frequency through the deployment of Frequency Selective Surfaces (FSS).

FSS are planar periodic structures consisting of identical thin conducting elements, usually printed on dielectric substrates. They behave as spatial electromagnetic filters selectively reflecting or attenuating a desired frequency band. Investigation was focused on studying through CFDTD simulations and anechoic chamber measurements the behaviour of FSS when these are attached on conventional building materials. It was found that beyond a certain distance (one tenth of the wavelength) away from the wall, the frequency response of the FSS remains unchanged.

The potential benefits in signal coverage, interference reduction and capacity increase through a MIMO system have been studied through a custom written Hybrid Ray Tracing model, which incorporates the behaviour of frequency selective surfaces. It is to the author best knowledge that such a hybrid Ray Tracing model has never been proposed in open literature.

Key words: Radio Wave Propagation, Frequency Selective Surfaces, Passive Repeaters, Ray Tracing, Periodic Structures.

WWW: $\quad$ http://www.ee.surrey,ac.uk/CCSR/ 


\section{Acknowledgments}

I would like to express my sincere thanks to my supervisor Dr. Stavros Stavrou for his valuable and continuous guidance throughout the whole process of this research. I would also like to thank Prof. Barry Evans, head of CCSR, where I worked during 2004-2007. Special thanks go to my colleagues Ming Yang and Pete King for their help on various aspects of this research. They offered some enlightening insights on building periodicity and MIMO systems.

It would be unjust to omit my parents for their emotional and financial support throughout the whole period of my Ph.D. Special thanks to my friends, who offered a much needed distraction at some very hectic periods of this work. 


\section{Contents}

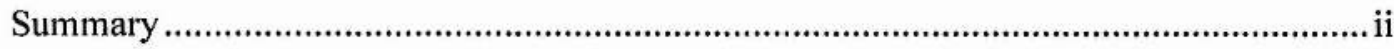

Acknowledgments .....................................................................................................iii

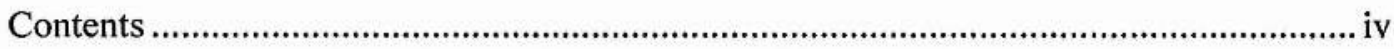

List of Figures ............................................................................................... viii

Glossary of Terms ….............................................................................................

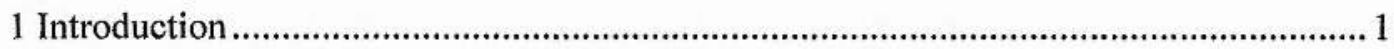

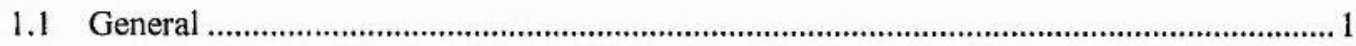

1.2 Aims and Objectives.............................................................................................

1.3 Structure of Thesis........................................................................................................

1.4 Achievements and contributions ...............................................................................

1.5 Other Contributions .....................................................................................................

2 Radio Wave Propagation Basics ..............................................................................

2.1 Maxwell's Equations .....................................................................................................

2.1.1 Plane wave solution and its properties....................................................................

2.2 Lossy media and constitutive parameters..................................................................... 10

2.3 Polarisation .................................................................................................................... 10

2.4 Radio Propagation Mechanisms ................................................................................11

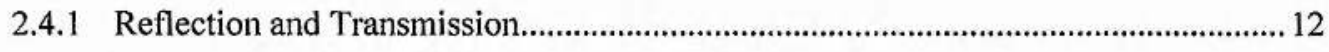

2.4.1.1 The Boundary Model........................................................................................ 13

2.4.1.2 Layer model (single slab model) ........................................................................ 16

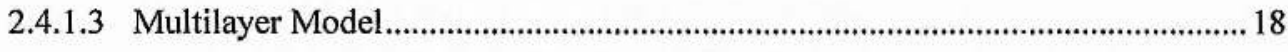

2.4.1.4 Ray Fixed Coordinate System ......................................................................20

2.4.1.5 Electric Field decomposition.........................................................................21

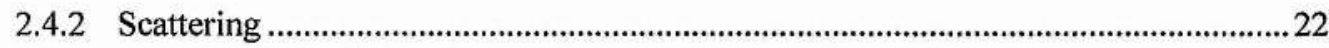

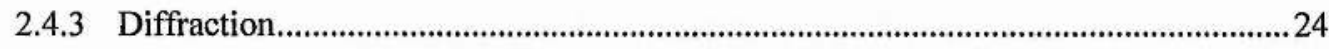

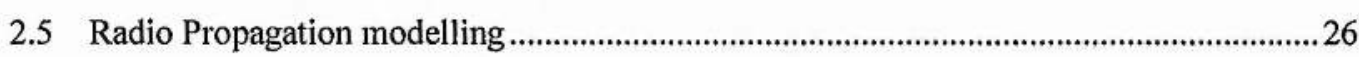

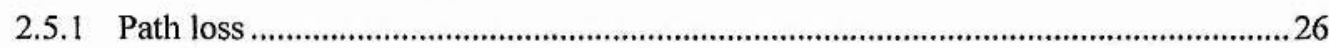

2.5.2 Empirical and Semi-Empirical Models.................................................................27

2.5.3 Deterministic or Site-Specific Models................................................................28 


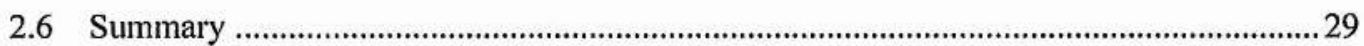

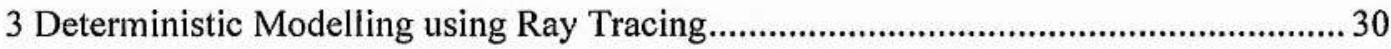

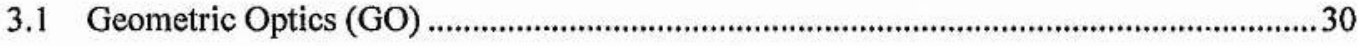

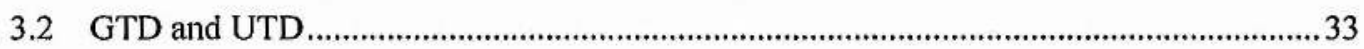

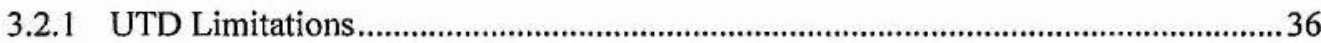

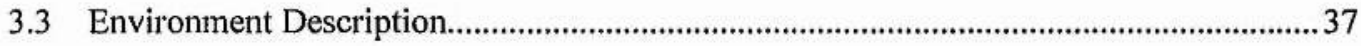

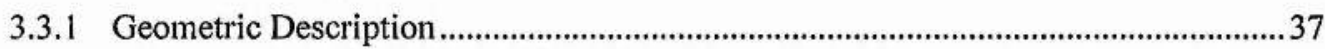

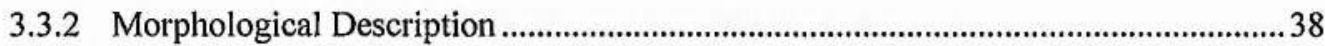

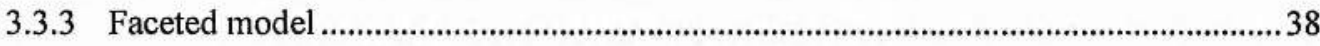

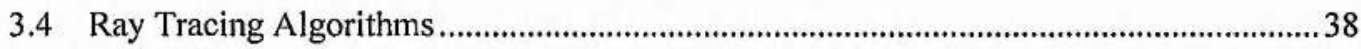

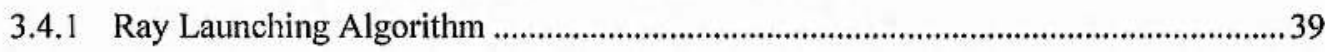

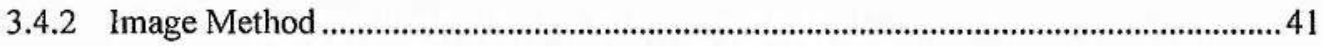

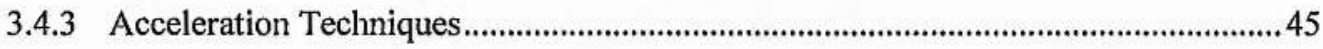

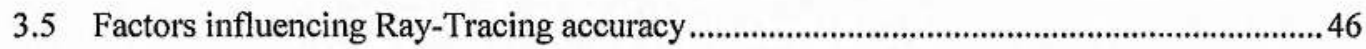

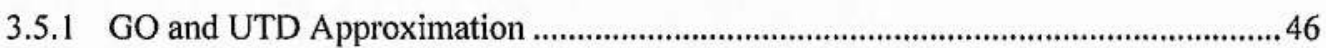

3.5.2 Algorithm Implementation and Propagation mechanism factors.................................47

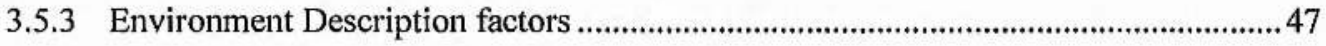

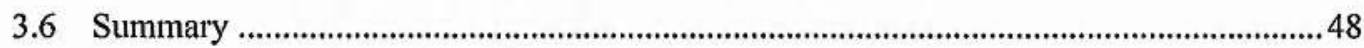

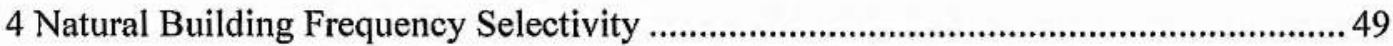

4.1 Rigorous Coupled Wave Analysis (RCWA) for periodic structures...................................50

4.1.1 Model Description .....................................................................................................5

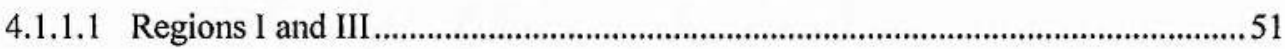

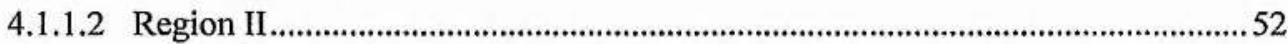

4.1.1.3 Truncation of Matrix Computation …….............................................................5

4.1.1.4 Boundary Conditions, Reflection and Transmission Coefficients ........................5

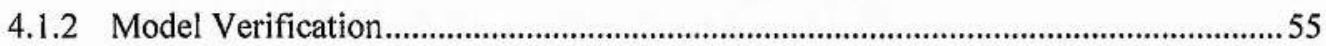

4.2 Concrete Masonry Block Structures.....................................................................................57

4.3 Reinforced Concrete Structures..................................................................................61

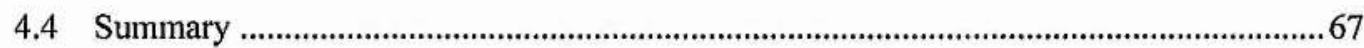

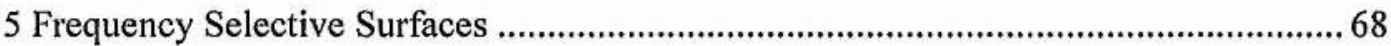

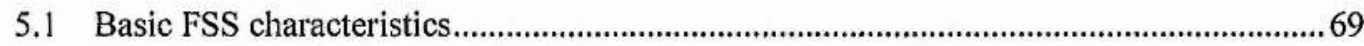

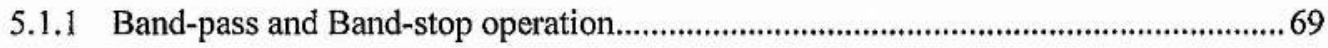

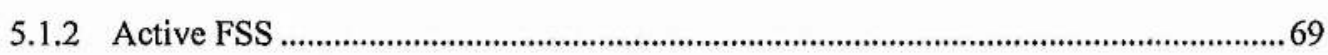

5.1.3 The supporting dielectric ……..................................................................................... 70 


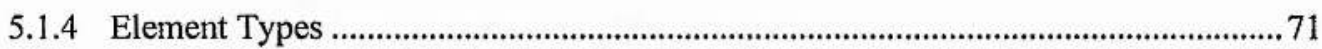

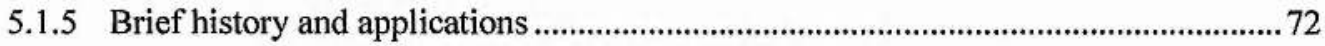

5.2 Techniques for analyzing Frequency Selective Surfaces ..................................................... 74

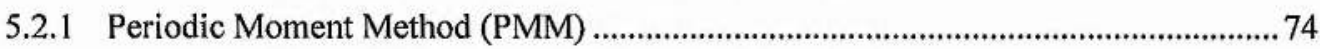

5.2.2 Finite Element method (FEM) …............................................................................... 74

5.2.3 Finite-Difference Time-Domain method (FDTD) ………............................................ 75

5.2.3.1 Conformal Finite-Difference Time-Domain Method (CFDTD) ...........................76

5.2.4 Spectral Domain Method (SDM) .................................................................................

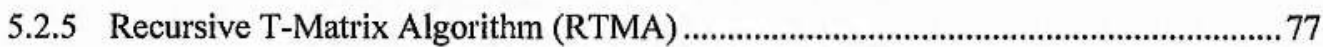

5.2.6 Equivalent Circuit Method (ECM) …................................................................... 77

5.3 Frequency Selective Surfaces on Building Materials ........................................................79

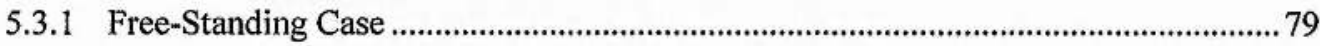

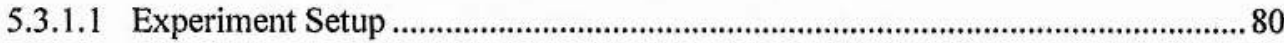

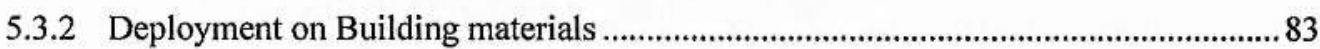

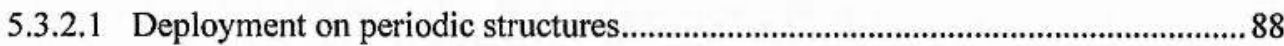

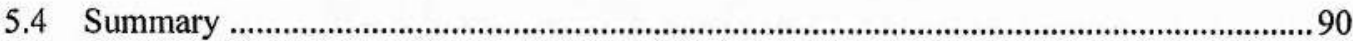

6 Modified Ray Tracing Model to account for FSS behaviour.......................................91

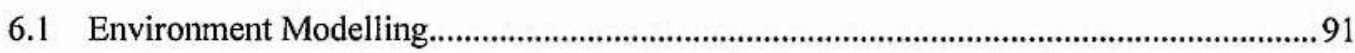

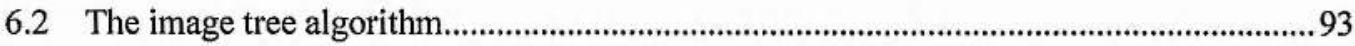

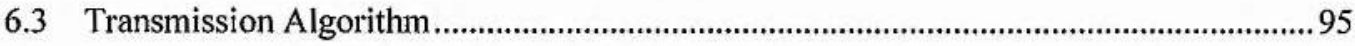

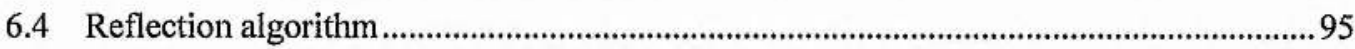

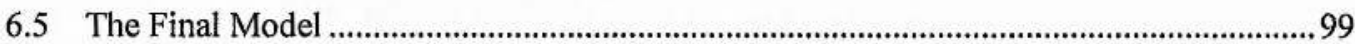

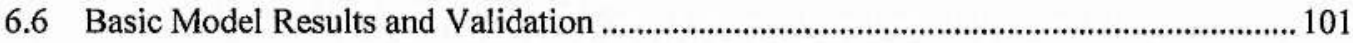

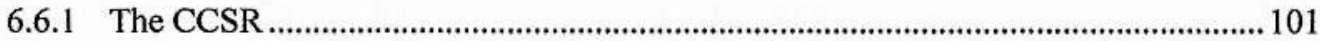

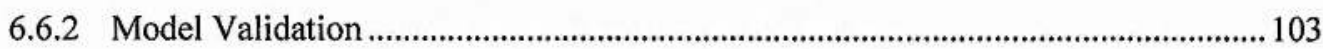

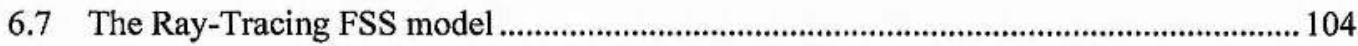

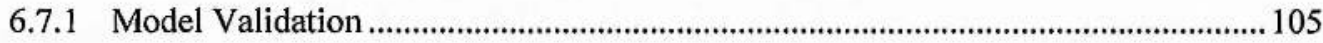

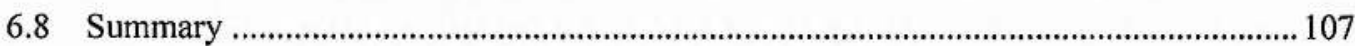

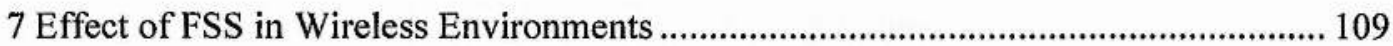

7.1 Initial Investigation-Feasibility Study ................................................................................. 110

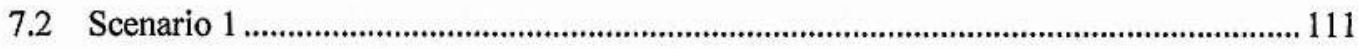

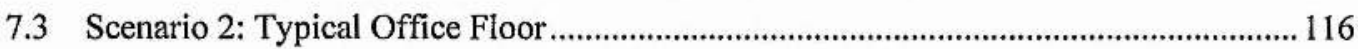

7.4 Scenario 3: Outdoor to Indoor-Operation of various wireless systems .............................119

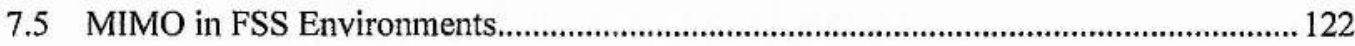

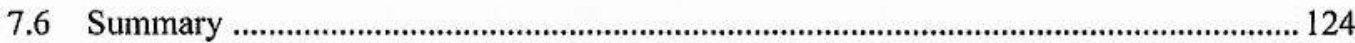




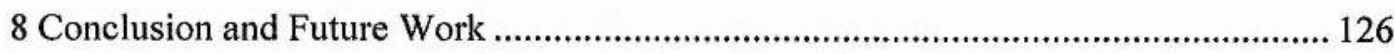

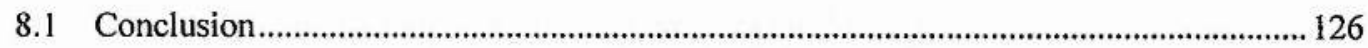

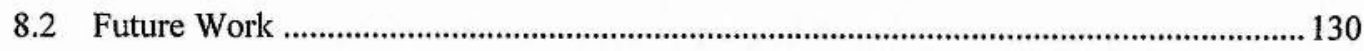

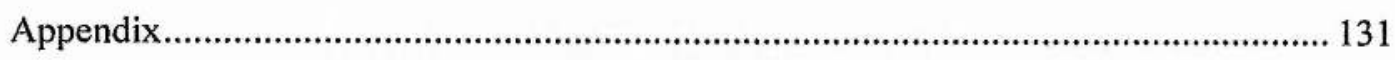

Appendix 1: EMCO MODEL 3115 Double Ridge Guide Antenna ........................................ 131

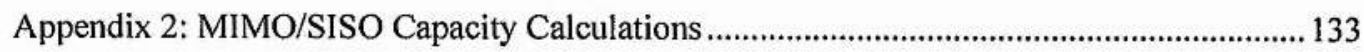

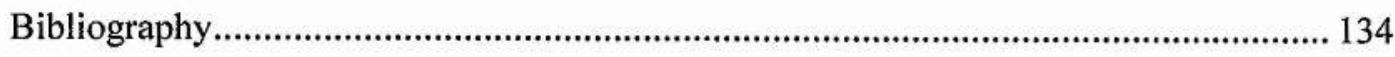




\section{List of Figures}

Figure 2-1: Plane wave 9

Figure 2-2: Polarisation states for waves propagating along the $\mathrm{z}$-axis ..........................................11

Figure 2-3: Reflection and Transmission form a plane interface .................................................. 12

Figure 2-4: Boundary Model for both polarisations for an incident plane wave............................. 14

Figure 2-5: Fresnel Reflection and Transmission coefficients for a plane wave incident on a half space dielectric medium with relative permittivity $\varepsilon_{2}=3$

Figure 2-6: (a) Single layer model (b) Difference in ray paths lengths for various rays emerging from the layer

Figure 2-7: Magnitude and Phase of the Transmission coefficient as a function of the internal to

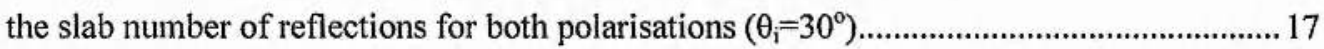

Figure 2-8: Boundary value solution for an n-layer dielectric wall............................................... 19

Figure 2-9: Three Dimensional Reflection and Transmission...........................................................2 20

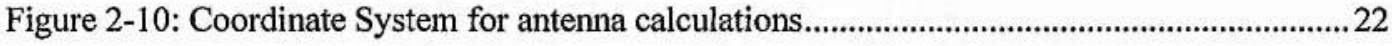

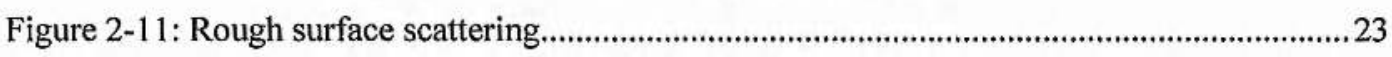

Figure 2-12: Rayleigh Criterion for surface roughness ...............................................................23

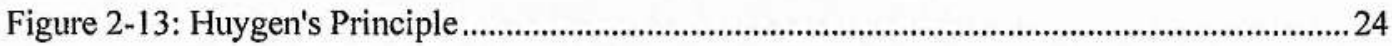

Figure 2-14: Huygen's Principle for knife-edge diffraction ............................................................25

Figure 3-1: Ray propagating through two successive wavefronts..................................................31

Figure 3-2: Generation of edge-diffracted rays ................................................................................ 33

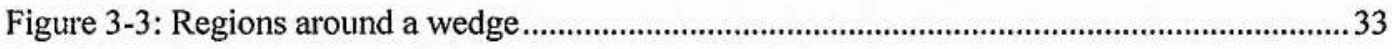

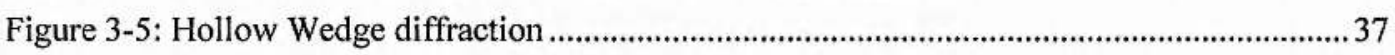

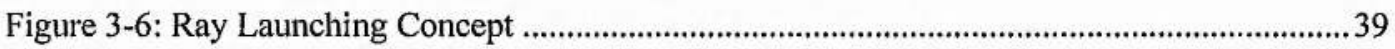

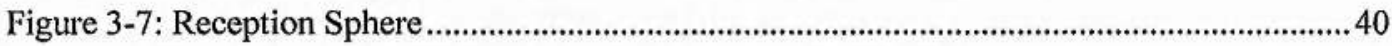

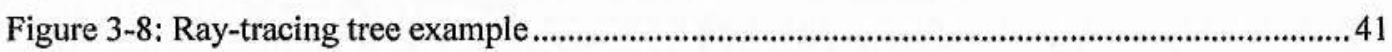

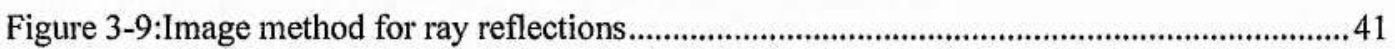

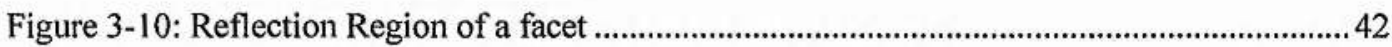

Figure 3-11: Image method for double reflections ..........................................................................4 43

Figure 3-12: (a) Image discarded by hiding (b) Facet 2 out of reflection space of Facet 1.............4 44

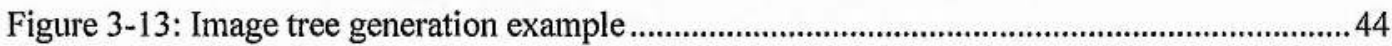

Figure 4-1: Geometry of 3D oblique incidence on a three-layer periodic structure.........................50

Figure 4-2: Convergence study of transmission loss versus the number of modes for the structure

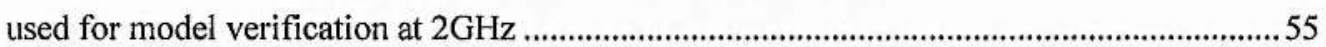

Figure 4-4: Masonry Block ...................................................................................................57 
Figure 4-5: Effect of neglecting the slot and cutout for an 8-inch masonry block at normal incidence.

Figure 4-6: Convergence Study for an 8-inch masonry Concrete block at $3 \mathrm{GHz}$.

Figure 4-8: Transmission loss of various propagating modes, $\left(\theta=37^{\circ}, \phi=0^{\circ}\right.$, TE pol.) for a masonry block wall

Figure 4-9: Transmission angles of various modes for various frequencies for masonry block wall

Figure 4-10: Transmitted and reflected modes for $\left(\boldsymbol{\theta}=37^{\circ}, \boldsymbol{\phi}=0^{\circ} \mathrm{TE}\right.$ pol. $)$ at $2 \mathrm{GHz} \ldots \ldots \ldots \ldots \ldots . . . . . . . . .61$

Figure 4-11: Reinforced Concrete

Figure 4-12: Convergence Study for a $20 \mathrm{~cm}$ thick reinforced concrete wall at $3 \mathrm{GHz}$ (steel bar periodicity $=15 \mathrm{~cm}$ )

Figure 4-13: Transmission/Reflection Coefficients for a 20cm-thick Reinforced Concrete wall $\left(\theta=37^{\circ}, \phi=0^{\circ}\right.$, TE pol. $)$.

Figure 4-14: Effect of steel rod periodicity (d) on the transmission loss of a $20 \mathrm{~cm}$-thick

Reinforced concrete $\left(\boldsymbol{\theta}=37^{\circ}, \boldsymbol{\phi}=0^{\circ}\right.$, TE pol. $)$......

Figure 4-15: Effect of a reinforced concrete wall thickness $(\mathrm{H})$ on the transmission loss $\left(\boldsymbol{\theta}=37^{\circ}\right.$,

$\phi=0^{\circ}, \mathrm{TE}$ pol., $\left.\mathrm{d}=15 \mathrm{~cm}\right)$.

Figure 4-16: Transmission loss of various propagating modes, $\left(\theta=37^{\circ}, \phi=0^{\circ} \mathrm{TE}\right.$ pol. $)$ for a

reinforced concrete wall

Figure 4-17: Transmission angles of various modes at various frequencies for a reinforced concrete wall.

Figure 4-18: Transmitted and reflected modes for $\left(\boldsymbol{\theta}=37^{\circ}, \boldsymbol{\phi}=0^{\circ} \mathrm{TE}\right.$ pol. $)$ at $2 \mathrm{GHz}$ from a reinforced concrete wall 66

Figure 5-1: Typical Frequency Selective Surface (Cross Dipole Design).

Figure 5-2: Equivalent Circuits of (a) a periodic surface of infinitely long rods and (b) a periodic surface of finite conducting elements.

Figure 5-3: Complementary arrays of Circular Loop FSS

Figure 5-4: Active Square Loop FSS with PIN diodes ( $d=7 \mathrm{~mm}, \mathrm{~g}=3.2 \mathrm{~mm}$, $\mathrm{w}=1 \mathrm{~mm}$ on FR4 dielectric with $\left.\varepsilon_{\mathrm{T}}=4.55\right)$

Figure 5-5: Effect of the dielectric thickness on a cross dipole FSS. (a) Dielectric only on one side

(b) dielectric on both sides of the elements .71

Figure 5-6: Unit cells of the basic FSS element geometries............................................................72

Figure 5-7: Use of FSS as a Cassegrain Sub-reflector ......................................................................73

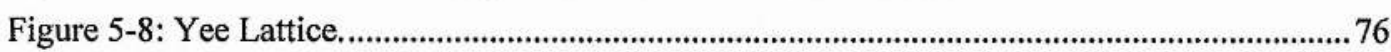

Figure 5-9: Equivalent Circuit Model of a square loop FSS ....................................................... 78 
Figure 5-10: Comparison between the Equivalent Circuit method (ECM) and the CFDTD method for a square loop FSS at normal incidence .79

Figure 5-11: Anechoic Chamber setup for FSS transmission loss measurements .......................... 80

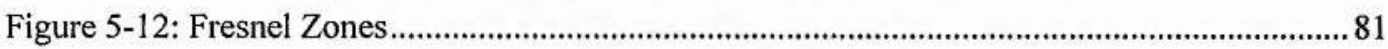

Figure 5-13: Transmission through a square-loop FSS under normal angle of incidence. .81

Figure 5-14 : Transmission (S21) and Reflection (S11) loss of the square loop FSS for various angles of incidence. (a) TE polarisation (b) TM polarisation................................................8 82

Figure 5-15: Relative permittivity of plaster and MDF (wooden) boards......................................8

Figure 5-16: Loss Tangent of plaster and MDF (wooden) boards .................................................. 84

Figure 5-17: Effect of wood and plaster when these are attached on the square loop FSS .............85

Figure 5-18: Effect of varying the air gap distance between the square loop FSS and a $9 \mathrm{~mm}$ wooden board at normal incidence

Figure 5-19: Simulation and measurement of the effect of the air gap between the square-loop

FSS and a 9mm-thick wooden (MDF) board on the tuning frequency.

Figure 5-20: Air gap effect for various MDF thicknesses on the square loop FSS response ..........87

Figure 5-21: Air gap effect for various $20 \mathrm{~mm}$-thick building materials .87

Figure 5-22: Air gap effect for various FSS designs at 2.4 and 5.2 GHz deployed on 20mm-thick MDF 88

Figure 5-23: Reinforced concrete geometry for FSS deployment ................................................. 88

Figure 5-24: Effect of a reinforced concrete wall on the square-loop FSS frequency response ....89

Figure 5-25: Effect of the air gap between a reinforced concrete wall and a square loop FSS on the tuning frequency. .89

Figure 6-1: Ray Tracing Algorithm Environment Description .................................................92

Figure 6-2: Image structure example ............................................................................................93

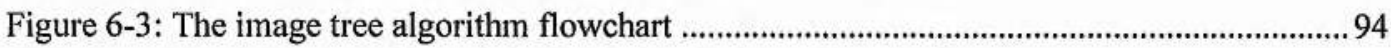

Figure 6-4 : Transmission Algorithm Flowchart ........................................................................96

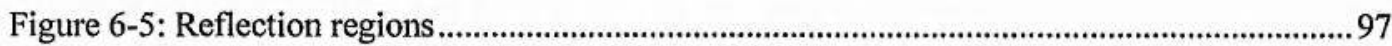

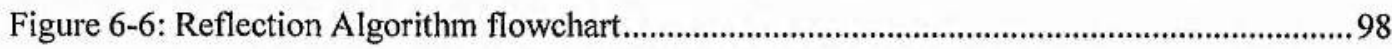

Figure 6-7: Diffraction effect. (a)Only reflection/refraction considered, (b) diffraction contribution, (c)reflection/refraction/diffraction considered ……….......................................99

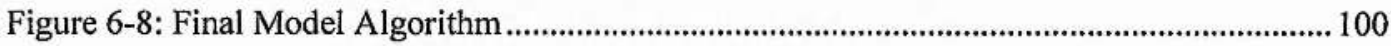

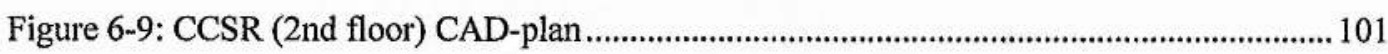

Figure 6-10: Environmental description of the $2^{\text {nd }}$ floor of the CCSR building.............................. 102

Figure 6-11: CCSR 2 $2^{\text {nd }}$ floor field prediction at $2.412 \mathrm{GHz}$.......................................................... 103

Figure 6-12: Comparison between measurements and modelling.................................................. 104

Figure 6-13: Anechoic Chamber Experiment Setup (internal view) .......................................... 105 
Figure 6-14: Transmission (S21) and Reflection (S11) coefficients of a square-loop FSS under various angles of incidence as suggested by the CFDTD method........................................... 106

Figure 6-15: Anechoic Chamber results for the 3 cases under investigation ................................107

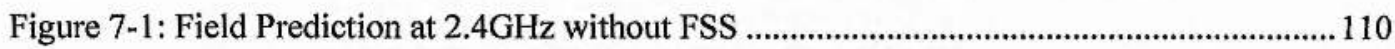

Figure 7-2: Field Prediction at $2.4 \mathrm{GHz}$ with FSS replacing the external walls only ....................110

Figure 7-3: Field prediction at $2.4 \mathrm{GHz}$ with FSS added on external and internal walls ...............111

Figure 7-5: Square loop FSS tuned at $2.4 \mathrm{GHz}$ deployed on building walls ................................ 113

Figure 7-6: Transmission loss for square loop FSS free standing case and the case where it is

placed $12.5 \mathrm{~mm}$ in front of plaster........................................................................................... 113

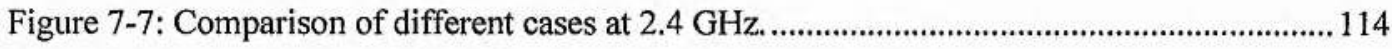

Figure 7-8: Effect of a $2.4 \mathrm{GHz}$ tuned FSS on a $5.2 \mathrm{GHz}$ wireless system (scenario 1).................115

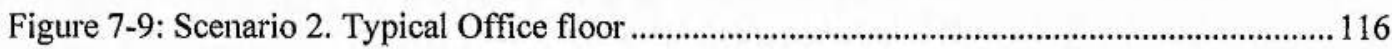

Figure 7-10: Comparison of different cases at $2.4 \mathrm{GHz}$ for scenario 2. The letters at the top of the

figure correspond to the points along the estimation route shown in Figure 7-9 .................117

Figure 7-11: The Effect of a $2.4 \mathrm{GHz}$ tuned FSS on a $5.2 \mathrm{GHz}$ wireless system (scenario 2).......118

Figure 7-12: Outdoor to Indoor and Indoor to Outdoor Scenario ................................................... 119

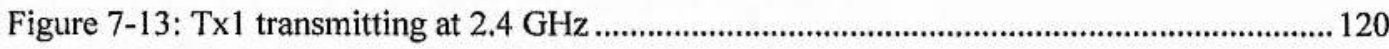

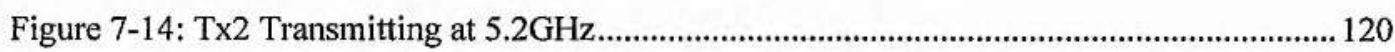

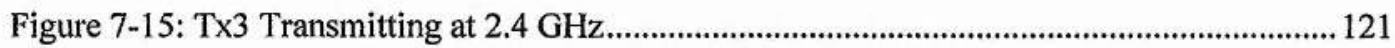

Figure 7-16: Cellular Base Station Transmitting at 900MHz....................................................... 121

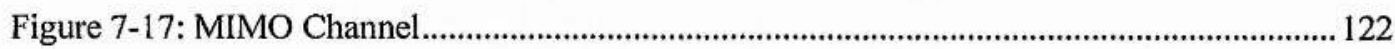

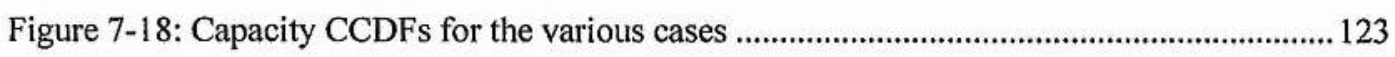

Figure 7-19: Capacity Multiplier CCDFs for the various cases .................................................. 124 


\section{Glossary of Terms}

\begin{tabular}{|c|c|}
\hline $\mathrm{ABC}$ & Absorbing Boundary Condition \\
\hline CFDTD & Conformal Finite Difference Time Domain \\
\hline $\mathrm{CW}$ & Coplanar Wave \\
\hline ECM & Equivalent Circuit Method \\
\hline $\mathrm{EM}$ & Electromagnetic \\
\hline FDTD & Finite Difference Time Domain \\
\hline FEM & Finite Element Method \\
\hline FSS & Frequency Selective Surface \\
\hline GO & Geometric Optics \\
\hline GTD & Geometrical Theory of Diffraction \\
\hline GSM & Global System for Mobile \\
\hline IE & Integral Equation \\
\hline LOS & Line of Sight \\
\hline Mbps & Megabits per second \\
\hline MDF & Medium Density Fibreboard \\
\hline MIMO & Multiple Input Multiple Output \\
\hline MTL & Modal Transmission Line \\
\hline NLOS & Non Line of Sight \\
\hline OFDM & Orthogonal Frequency Domain Multiplexing \\
\hline $\mathrm{PBC}$ & Periodic Boundary Condition \\
\hline PDE & Partial Differential Equation \\
\hline PER & Packet Error Rate \\
\hline PMM & Periodic Moment Method \\
\hline QoS & Quality of Service \\
\hline RCS & Radar Cross Section \\
\hline RCWA & Rigorous Coupled Wave Analysis \\
\hline RTMA & Recursive T-Matrix Analysis \\
\hline $\mathrm{Rx}$ & Receiver \\
\hline SBR & Shooting and Bouncing Ray \\
\hline SDM & Spectral Domain Method \\
\hline SISO & Single Input Single Output \\
\hline SNR & Signal to Noise Ratio \\
\hline STBC & Space Time Block Code \\
\hline
\end{tabular}


TE

TM

$\mathrm{Tx}$

UTD

WiMAX

WLAN
Transverse Electric

Transverse Magnetic

Transmitter

Uniform Theory of Diffraction

Worldwide Interoperability for Microwave Access

Wireless Local Area Network 


\section{Chapter 1}

\section{Introduction}

\subsection{General}

In recent years the wireless communication market has experienced an explosive growth. The first analogue wireless communication system, which utilised FM modulation, appeared in the 1980 s leading to the first digital cellular (2G) system in the early 1990s. The introduction of this system significantly improved the spectral efficiency through the use of the cellular concept which is based on the frequency reuse idea. Nowadays, third generation (3G) mobile communication systems are starting to evolve offering a range of voice, data and multimedia services. In addition to that, research is being currently carried out for fourth generation (4G) systems [1], which will provide an all-IP network that integrates the current services and provides new broadcast, cellular, cordless, WLAN and short-range communication.

In the highly competitive communication industry such networks need to be designed and implemented very quickly and at the lowest cost. Too much effort is put by researchers in order to improve the radio propagation conditions in such a way as to increase radio coverage and reliability, meet the increased traffic demands and provide a high-quality signal to the higher layers of the OSI model. Several techniques have been proposed over the years for improving radio propagation in indoor and outdoor communication. However, it is common practice that such methods are first of all simulated before they are put in place. Thus, developing an accurate propagation tool is extremely important in terms of system planning.

A large number of empirical and semi-empirical models have been proposed or deployed over the years in order to predict radio coverage for indoor and outdoor communications. However these models do not always provide accurate results in cases where the cell size is relatively small and the channel behaviour is unpredictable due to the frequently changing propagation clutter. This would mean that empirical or semi-empirical models, which apply only for scenarios which are very similar to the ones that the original measurements have been performed in [4], would fail to provide a sufficient prediction. In addition to that, and since microcellular and indoor communication systems continue to evolve very rapidly there is a growing demand for accurate propagation models. The deployment of high speed WLANs in modern office buildings has enhanced the need for this kind of models, where detailed radio 
coverage is necessary to assure complete coverage without interference between adjacent networks and effectively assure good Quality of Service (QoS) and efficient use of the available spectrum. Such models are called deterministic or site-specific since they take into account all the possible contributions arising from a vast number of propagated rays. This means that all the geometrical and electrical properties of a particular propagation environment are taken into consideration. Ray tracing is the dominant technique for developing deterministic (twodimensional or three dimensional) propagation models. This technique identifies the dominant ray paths reaching the receiver location and determines their field strength using electromagnetic theory.

It is possible that in modern office buildings two or more collocated (not necessarily in the same building) WLANs operate at the same time and on the same frequency channel. In such cases, what needs to be assured is that the interference between these networks is minimised. A way to achieve this is to deploy Frequency Selective Surfaces (FSS) on different interfaces. These surfaces can act as spatially deployed RF filters, providing isolation between different areas while at the same time can channel the signal to other areas. This leads to the novel idea of frequency selective buildings and the investigation of radio propagation in such frequency selective environments.

Prior to any FSS investigation it would be wise to study the "natural frequency building behaviour". Building interfaces can be non-homogeneous leading to the generation of nonspecular components after an interaction of the propagating wave with the respective interface. In fact, in many cases, building interfaces exhibit a periodic behaviour (i.e. reinforced concrete and masonry block walls with air-pockets ) which gives rise to the propagation of higher order modes (harmonics) under various non-specular directions and also present some frequency selectivity (or frequency tuning).

Various factors need to be considered when designing a Frequency Selective environment by using Frequency Selective Surfaces. These include polarisation issues, angular sensitivity and factors accounting the actual deployment of FSS on the building walls. It has been shown that when a periodic surface such an FSS is deployed directly on any dielectric medium, there is a shift of its resonance frequency which seems to depend on the dielectric properties of the medium that the surface is deployed on [65]. A way to overcome this problem is to increase the airgap between the FSS and the building material, so that the two (FSS and wall) behave as independent facets, affecting the propagation of waves that interact with them in an independent way as two separate processes. 


\subsection{Aims and Objectives}

The aim of this research was to propose novel techniques for improving radio propagation in indoor and outdoor wireless environments in such a way as to increase radio coverage and reduce interference from systems operating in the close vicinity of our system. The basic idea was to transform the buildings' interfaces into- frequency selective ones, which have special characteristics in terms of reflection and refraction of the propagating waves at the frequency of interest. This research investigates the use of Frequency Selective Surfaces in wireless environments, their modelling behaviour and the effect of periodic structures in an outdoor and/or indoor environment.

A sequential approach was used to carry out this investigation, where the following intermediate objectives were identified, corresponding to the different stages of the investigation.

- To study the radio wave propagation mechanisms in a wireless propagation channel and to carry out a comprehensive literature survey on the existing radio propagation models.

- To review radio propagation modelling and develop a deterministic ray-tracing model, looking into the fundamental limitations of existing algorithms and the main factors influencing its accuracy.

- To characterise the building natural behaviour when interacting with electromagnetic waves considering that the building walls exhibit an internal periodic behaviour by utilising the Rigorous Coupled Wave Analysis (RCWA).

- To theoretically and experimentally study the various factors governing the operation of Frequency Selective Surfaces and investigate the effect on their radio propagation characteristics when they are deployed on conventional homogeneous and nonhomogeneous (periodic) building materials.

- Modify the developed Ray-Tracing model to incorporate the FSS behaviour and verify it in a small scale controlled environment (i.e. anechoic chamber). Examine FSS deployment in indoor and outdoor wireless environments in terms of signal coverage, isolation, and wave guiding effects at the frequency of interest. 


\subsection{Structure of Thesis}

This thesis consists of 8 chapters:

Chapter 2 gives an introductive review of the basic electromagnetic wave propagation principles, covering the various radio wave propagation mechanisms such as path loss, reflection, refraction, diffraction and scattering. It also gives a brief introduction into the basic radio propagation modelling techniques.

Chapter 3 reviews the basic principles and algorithms of Ray Tracing, and the high frequency methods used to reduce the complexity of the electromagnetic problem to be solved (Geometrical Optics GO and its extensions: Geometrical theory of Diffraction GTD and Uniform Theory of Diffraction UTD).

Chapter 4 presents the theory and development of the RCWA method to study the "natural building frequency behaviour" when the building interfaces exhibit a periodic behaviour which can give rise to higher propagation modes and resonances.

Chapter 5 deals with the basic principles governing the operation and analysis of FSS. It also presents, through simulations and anechoic chamber measurements, the interaction of FSS with typical building materials. This interaction plays an important role in the design of frequency selective wireless environments through the use of Frequency Selective Surfaces.

Chapter 6 presents the implemented Ray-Tracing algorithm and compares its results with results obtained using a commercial simulator and measurements carried out in an indoor office environment (CCSR). It also verifies the applicability of the modified Ray Tracing model which incorporates the FSS behaviour through measurements carried out in a small-scale indoor environment constructed in an anechoic chamber.

Chapter 7 presents FSS Ray-Tracing simulations results for various indoor and outdoor scenarios that show that frequency selective surfaces can be utilised as passive repeaters to achieve various objectives regarding signal coverage and interference reduction. For these simulation results various factors governing the FSS propagation characteristics such as the angle of incidence, polarisation and the interaction of FSS with the building materials have been taken into account. Also an initial flavour of the capacity increase from utilising FSS in an indoor MIMO and SISO wireless environment is demonstrated.

Chapter 8 draws some conclusions on this particular research work and presents some future steps. 


\subsection{Achievements and contributions}

- Investigation of the effect of periodic structure of typical building walls on radio wave propagation, through a custom written RCWA method for predicting plane wave propagation through periodic building structures.

- Extensive theoretical and experimental investigation of the effect of deploying FSS on conventional homogeneous and periodic structures.

- Proposal of the "safe" air-gap distance that FSS can be deployed away from building materials so that their frequency response is not affected.

- Development and verification of a modified Ray-Tracing model to account for the deployment of FSS on building interfaces.

- Suggestion of the potential advantages from deploying FSS in indoor and outdoor wireless environments.

The results have been published in journals and one international conference.

\section{Journals/Transactions}

- M. Raspopoulos, F. A. Chaudhry, S. Stavrou, "Radio Propagation in Frequency Selective Buildings", Euro. Trans. Telecoms, Vol. 17, pp. 407-413, March 2006. (Invited Paper)

- M. Raspopoulos, S. Stavrou, "Frequency Selective Surfaces on Building Materials - Air gap Impact", IET Electronic Letters, Vol. 43, Issue 13, pp. 700-702, June 2007.

- M.Raspopoulos, S.Stavrou, "On the capacity of MIMO Systems in FSS environments", IET Electronic Letters, Accepted to be published.

- M. Raspopoulos, S. Stavrou, "Frequency Selective Buildings through Frequency Selective Surfaces", Submitted to IEEE Trans. Antennas Propag, Accepted, to be published.

\section{Conferences}

- F. A. Chaudhry, M. Raspopoulos, S. Stavrou, "Effect of Frequency Selective Surfaces on radio wave propagation in indoor environments", $11^{\text {th }}$ European Wireless Conf. 2005, Nicosia, Cyprus, Vol. 2, pp. 732-736, April 2005. (Best Paper Award)

\section{Book Contribution}

- M. Raspopoulos, a co-author in e-book, "Influence of the propagation channel on satellite communications - channel dynamic effects on mobile, fixed and optical multimedia applications", written as final deliverable for joint activity group on satellite propagation during EU IST FP6 SatNex project: Satellite Communications Network of Excellence. 


\subsection{Other Contributions}

Contributed in the following work and reports:

- "Optimizing radio tactical positions," a project sponsored by Defence Science and Technology Laboratory (DSTL), Ministry of Defence, UK, Mar. 2006.

- "WLAN deployment in complex aircraft environments", a project sponsored by RollsRoyce plc.

- "Investigating radio interference between MSS and ATC", a project sponsored by INMARSAT Ltd. 


\section{Chapter 2}

\section{Radio Wave Propagation Basics}

When considering antennas and radio wave propagation problems, some basic knowledge of the properties of electromagnetic waves, which travel either in free space or in any other uniform media, is required in order to establish the key parameters and relationships. Four types of propagation mechanisms exist that can describe the interaction of the electromagnetic waves with features of the environment. These are reflection, refraction (or transmission), scattering and diffraction and they all affect the amplitude, direction and phase of the propagating waves. This chapter gives a brief introduction into these principles, starting from the most fundamental electromagnetic wave laws also known as Maxwell's Equations.

\subsection{Maxwell's Equations}

In 1865, Maxwell combined the theoretical concepts described by a set of basic laws during the $19^{\text {th }}$ century by many scientists; Faraday, Ampere, Gauss and others, into a consistent set of vector equations. These four fundamental equations specify the relationships between the variations of the vector electric field $\boldsymbol{E}$ (Volts/metre) and the vector magnetic field $\boldsymbol{H}$ (Amperes/metre) at any point in space at any time. These equations can be expressed in either differential or integral form as follows [2][3],

Differential Form Integral Form

$$
\begin{aligned}
& \nabla \times E=-\frac{\partial \mathrm{B}}{\partial \mathrm{t}} \\
& \oint_{C} E \cdot d l=-\int_{S} \frac{\partial B}{\partial t} \cdot d A \\
& \nabla \times H=\frac{\partial \mathrm{D}}{\partial \mathrm{t}}+\mathrm{J} \\
& \oint_{C} H \cdot d l=\int_{S} \frac{\partial D}{\partial t} \cdot d A+\int_{S} J \cdot d A \\
& D=\rho \\
& \nabla \cdot B=0 \\
& \oint_{S} D \cdot d A=q=-\int_{V} \rho \cdot d V \\
& \oint_{S} B \cdot d A=0
\end{aligned}
$$


where $\mathbf{E}$ is the electric field $(\mathrm{V} / \mathrm{m}), \mathbf{H}$ the magnetic field $(\mathrm{A} / \mathrm{m}), \mathbf{B}$ the magnetic flux density (weber $/ \mathrm{metre}$ or tesla), $\mathrm{J}$ the current density $\left(\mathrm{A} / \mathrm{m}^{2}\right), \rho$ the electric charge density (coulomb $\left./ \mathrm{m}^{3}\right), \mathbf{D}$ the electric displacement field (coulomb $/ \mathrm{m}^{2}$ ), $\mathbf{d A}$ the differential vector element of surface area A with very small magnitude and direction normal to surface $S, \mathbf{d V}$ the differential element volume $\mathrm{V}$ enclosed by $\mathrm{S}$ and $\mathbf{d l}$ is the differential vector element of path length tangential to contour $\mathrm{C}$ enclosed by surface S. Also $\boldsymbol{\nabla} \cdot$ is the divergence operator and $\boldsymbol{\nabla} \times$ is the curl operator. The above equations can be read as follows [4]:

Eq. (2.1) An electric field is produced by a time-varying magnetic field

Eq. (2.2) A magnetic field is produced by a time-varying electric field or by a current

Eq. (2.3) Electric field lines either start or end on charges, or are continuous.

Eq. (2.4) Magnetic field lines are continuous

\subsubsection{Plane wave solution and its properties}

Several solutions of the aforementioned Maxwell's Equations exist which can represent a field that can be actually produced in practice. These can all be represented as a sum of constant frequency waves also known as plane waves. The general behaviour of a wave as a function of time can be expressed as a superposition of waves at different frequencies through Fourier transform. Therefore, a convenient and sufficient solution is to examine the characteristics of a wave at a single frequency; also known as time-harmonic or monochromatic wave [3]. In a rectangular coordinate system, it can be shown that the plane wave solution satisfies [2]:

$$
\left(\nabla^{2}-\gamma^{2}\right) E_{x, y . z}^{i}=0
$$

$E_{x, y, z}^{i}$ is the scalar component of the electric field in any direction (x,y or $\mathrm{z}$ ) and $\gamma$ is the propagation constant which satisfies the following equation,

$$
\gamma=\sqrt{j \omega \mu(\sigma+j \omega \varepsilon)}=a \pm j \beta
$$

where $\boldsymbol{\alpha}$ and $\boldsymbol{\beta}$ are the attenuation constant $(\mathrm{Np} / \mathrm{m})$ and phase constant $(\mathrm{rad} / \mathrm{m})$ respectively. The terms $\mu, \sigma$ and $\varepsilon$ are the propagating medium constitutive parameters at the frequency of operation (see next section). The choice of the conjugate solution of $\gamma$ depends on the phase variation along the direction of propagation; + for increasing phase and - for decreasing phase. For the rest of this thesis propagation constant will be assumed to be $\gamma=a+j \beta$.

The plane wave solution can be found by using a separation of variables method [2]. This solution represents a uniform plane wave, propagating along the z-axis; also known as poynting vector, as shown in Figure 2-1. A uniform plane wave is the one that the field is not a function of the coordinates that form the equiphase and equiamplitude plane [2]. For such a plane wave the E- 
field and $\mathrm{H}$-field are perpendicular to each other and are both perpendicular to the poynting vector which describes the magnitude and direction of the power flow carried by the wave. Both fields are in phase at any point in space or time. The term plane wave arises from the fact that the wavefront (a surface of constant phase) forms a plane parallel to the $x y$ plane.

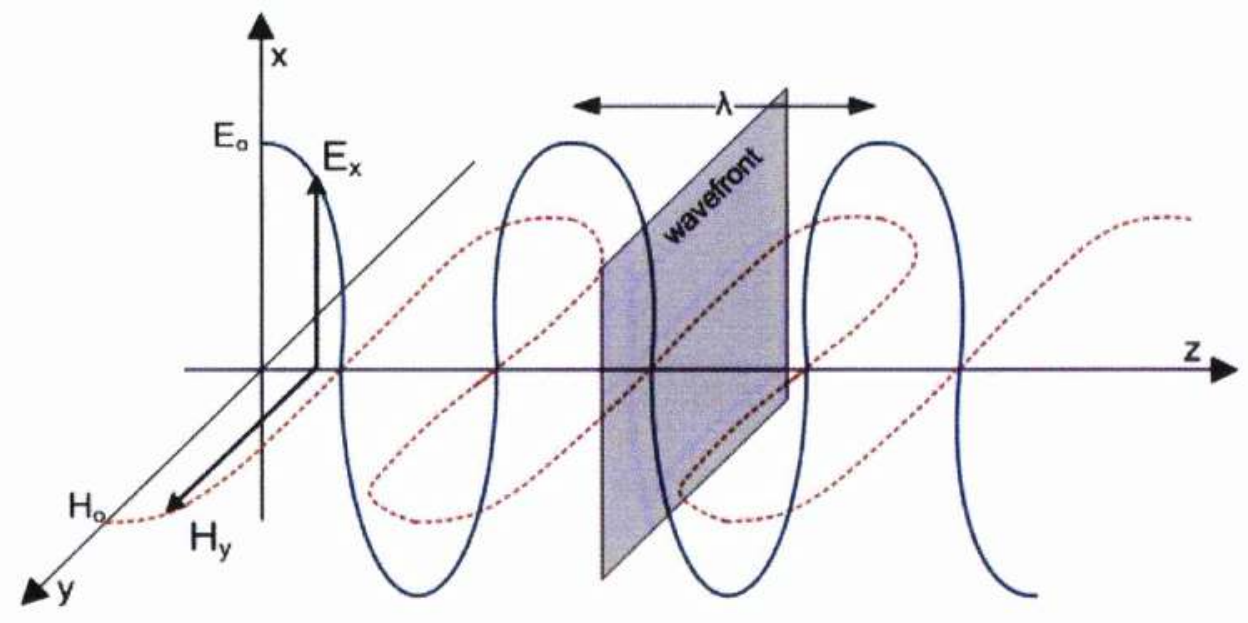

Figure 2-1: Plane wave

The electric field can be expressed using a complex expression,

$$
\vec{E}_{x}=E_{o} e^{-\gamma z} \hat{x}=E_{o} e^{-a z} e^{-j \beta z} \hat{x}
$$

where $E_{0}$ the electric field amplitude and $\hat{x}$ is a unit vector that describes the alignment of the electric field, relative to the direction of propagation, known as polarisation of the wave. For this particular example the wave is linearly polarised since the electric field vector has a single direction parallel to the $\mathrm{x}$-axis. Similarly the magnetic field can be expressed as:

$$
\vec{H}_{y}=H_{o} e^{-\gamma z} \hat{y}=H_{o} e^{-a z} e^{-j \beta z} \hat{y}
$$

The wave impedance for the propagating medium can be found as,

$$
Z=\frac{|\vec{E}|}{|\vec{H}|}=\frac{E_{o}}{H_{o}}=\sqrt{\frac{\mu}{\varepsilon}}
$$

It is of special interest to calculate the wave impedance in free space since it will be used in the calculation of the electric field in free space.

$$
Z_{o}=\sqrt{\frac{\mu_{o}}{\varepsilon_{o}}} \cong \sqrt{4 \pi \times 10^{-7} \times \frac{36 \pi}{10^{-9}}}=120 \pi \approx 377 \Omega
$$




\subsection{Lossy media and constitutive parameters}

When a wave interacts with a lossy medium, its energy diminishes with distance travelled through the medium. This necessitates the morphological specification of the medium properties by means of a set of unambiguous parameters. These parameters are called electrical or constitutive parameters. These parameters are: the electrical permittivity $\varepsilon(\mathrm{F} / \mathrm{m})$, the permeability $\boldsymbol{\mu}(\mathrm{H} / \mathrm{m})$ and the conductivity $\boldsymbol{\sigma}(\mathrm{S} / \mathrm{m})$. A very close, if not exact, knowledge of these parameters is essential to accurately model a radio propagation environment. Permittivity and permeability are normally expressed relative to the free space values as,

$$
\begin{aligned}
\varepsilon & =\varepsilon_{r} \varepsilon_{o} \\
\mu & =\mu_{r} \mu_{o}
\end{aligned}
$$

where $\varepsilon_{r}$ is the relative permittivity, $\mu_{r}$ is the relative permeability with $\varepsilon_{o}=8.854 \times 10^{-12} \mathrm{~F} / \mathrm{m}$ and $\mu_{o}=4 \pi \times 10^{-7} \mathrm{H} / \mathrm{m}$ to be their respective free space values. It becomes essential when dealing with radio propagation modelling to define the complex value of the relative permittivity. This is calculated as,

$$
\varepsilon_{\text {complex }}=\varepsilon_{r}(1-j \tan \delta)
$$

where $\tan \delta$ is the loss tangent defined as:

$$
\tan \delta=\frac{\sigma}{2 \pi f \varepsilon_{r} \varepsilon_{o}}
$$

The constitutive parameters can also be used to classify the material as good conductor or as good dielectric (insulator). When $(\sigma / \omega \varepsilon)^{2} \gg 1$ the material is classified as good insulator whereas when $(\sigma / \omega \varepsilon)^{2} \ll 1$ the material is classified as good dielectric. $\omega=2 \pi f$ is the angular frequency.

\subsection{Polarisation}

Polarisation is defined as the alignment of the electric field, relative to the direction of propagation. Referring back to Figure 2-1 the electric field is parallel to the $\mathrm{x}$-axis which means that the wave is $x$-polarised or vertically polarised. In the same way if the electric field was parallel to the y-axis, then the wave would be y-polarised or horizontally polarised. In these two cases the electric field has a single direction along the direction of propagation and the waves are described as linearly polarised. If two plane waves of equal magnitude are combined with $90^{\circ}$ phase difference the resulting wave will be circularly polarised. If the two components are of unequal amplitudes then the result is an elliptically polarised wave. Typical examples of waves 
propagating along the z-axis are shown in Figure 2-2. All these states can be represented by a compound electric field $\vec{E} E$ composed of x- and y-linearly polarised plane waves with amplitudes $\vec{E}_{x} \mathrm{E}$ and $\vec{E}_{y} \mathrm{E}$ as [4]:

$$
\vec{E}=\vec{E}_{x} \hat{x}+\vec{E}_{y} \hat{y}
$$
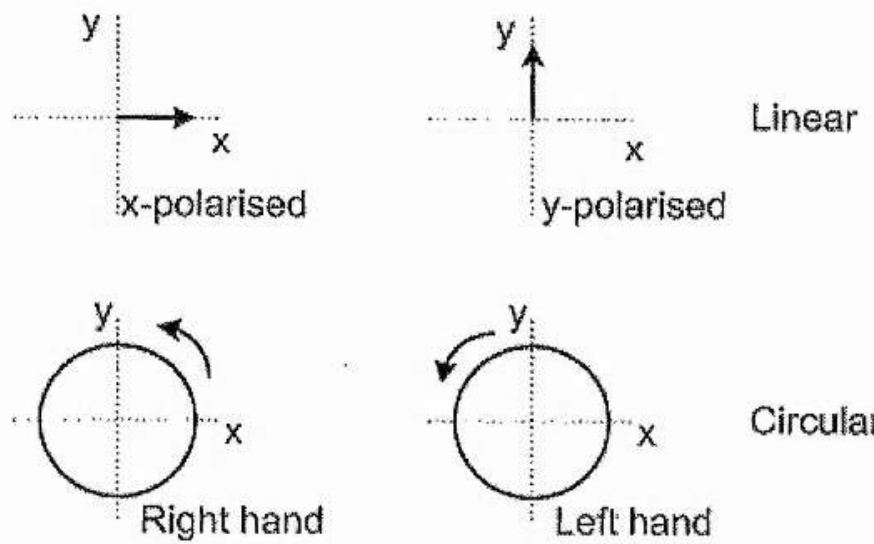

\section{Circular}

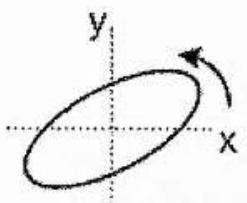

Right hand

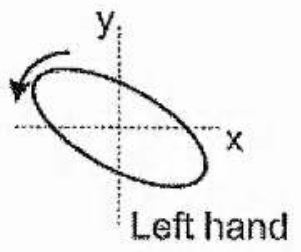

\section{Eliptical}

Figure 2-2: Polarisation states for waves propagating along the z-axis

\subsection{Radio Propagation Mechanisms}

Whenever a propagating wave impinges on an obstruction (or boundary) with different material parameters than the propagating medium, its amplitude, phase, direction and polarisation will change. The understanding of these mechanisms is of major importance in the implementation of a deterministic radio propagation prediction model.

At any obstruction, the electric and magnetic field quantities must satisfy certain boundary conditions [3]. That is, the tangential components of the electric and magnetic field must be continuous across the boundary. Mathematically, this can be expressed as,

$$
\begin{aligned}
& \hat{n} \times \vec{E}_{1}=\hat{n} \times \vec{E}_{2} \\
& \hat{n} \times \vec{H}_{1}=\hat{n} \times \vec{H}_{2}
\end{aligned}
$$


where $\hat{n}$ is the unit vector normal to the interface and $\left(\vec{E}_{1}, \vec{H}_{1}\right)$ and $\left(\vec{E}_{2}, \vec{H}_{2}\right)$ are the fields in mediums with $\varepsilon_{1}$ and $\varepsilon_{2}$ respectively. These boundary conditions define the effects of reflection and refraction (most commonly known as transmission).

\subsubsection{Reflection and Transmission}

Whenever a propagating wave impinges on an obstruction with different material parameters than the propagation medium, having also greater dimensions than the wavelength, reflection and transmission (through the material) will occur. For these propagation mechanisms, the basic rule that the wave follows is called Snell's Law of reflection and refraction.

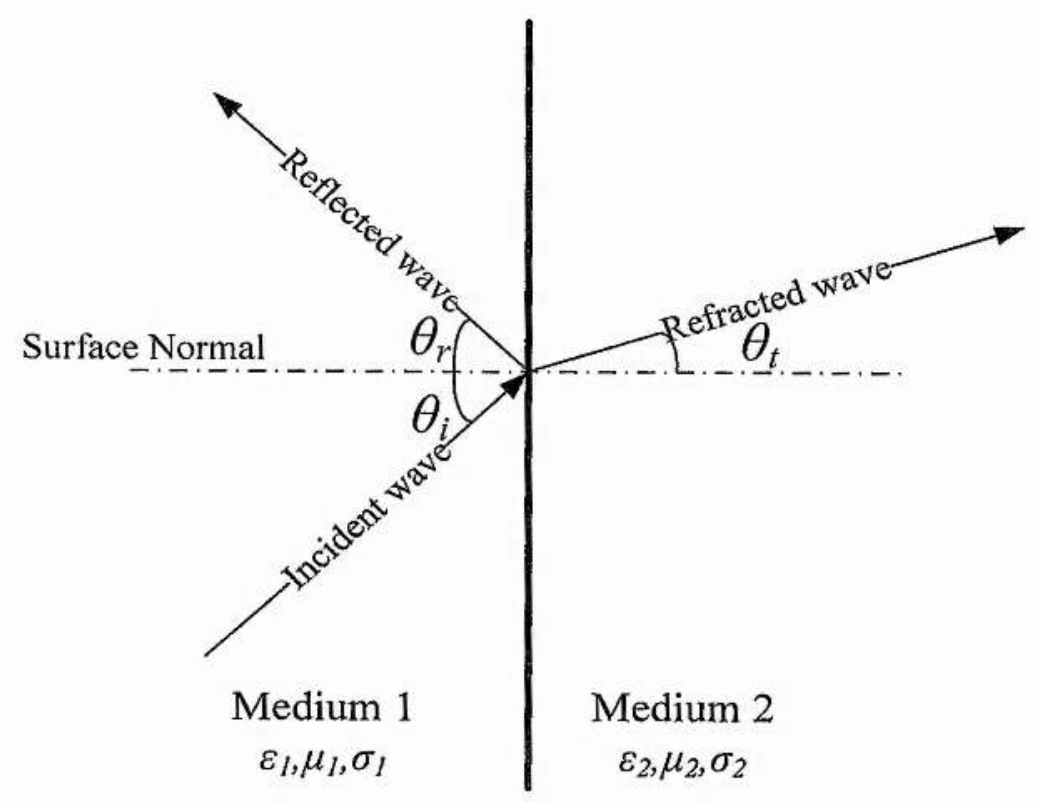

Figure 2-3: Reflection and Transmission form a plane interface

With reference to Figure 2-3, the Snell's Law of reflection states that the angle of the reflected field is equal to the angle of the incident field to the interface. That is:

$$
\theta_{i}=\theta_{r}
$$

The Snell's Law of refraction states that the refracted angle is a function of the incidence angle and the materials of the two media:

$$
\frac{\sin \left(\theta_{i}\right)}{\sin \left(\theta_{t}\right)}=\frac{n_{2}}{n_{1}}=n_{21}
$$

Where $n_{1}$ and $n_{2}$ are the refractive indices for the two propagating media. The refractive index is defined as: 


$$
n=\sqrt{\varepsilon_{r} \mu_{r}}
$$

Snell's laws mentioned above describe only the kinematic properties of the reflected and transmitted waves. In order to account for their dynamic properties, such as their amplitude, phase and polarisation the Fresnel Reflection and Transmission Coefficients need to be considered ( $\mathrm{R}$ and $T$ respectively). Using these coefficients the following equations apply,

$$
E_{r}=R E_{i} \text { and } E_{t}=T E_{i}
$$

where $E_{i}$ is the incident electric field, $E_{r}$ is the reflected electric field and $E_{t}$ is the transmitted electric field. There exist three mechanisms for calculating the reflection and transmission coefficients; the Boundary model [4], the Layer model [5] [6] and the Multi-layer model [8].

\subsubsection{The Boundary Model}

This model assumes that the two media are semi-infinite; medium 1 and medium 2 infinitely extent in the $-x$ and $+x$ direction respectively as shown in Figure 2-4. In order to calculate the reflected and the transmitted field, the Fresnel Coefficients of reflection and refraction can be used. These coefficients depend on the constitutive parameters of the materials, the angle of incidence and the polarisation of the incident wave. There are two possible polarisations of the incident plane wave that must be considered separately. One polarisation has the electric field vector perpendicular to the plane of incidence (perpendicular polarisation), sometimes called horizontal or transverse electric (TE) polarisation. The other has the electric field vector parallel to the plane of incidence (parallel polarisation), sometimes called vertical or transverse magnetic (TM) polarisation. The Fresnel reflection and transmission coefficients are different for cases when the incident plane wave is parallel or perpendicularly polarised.

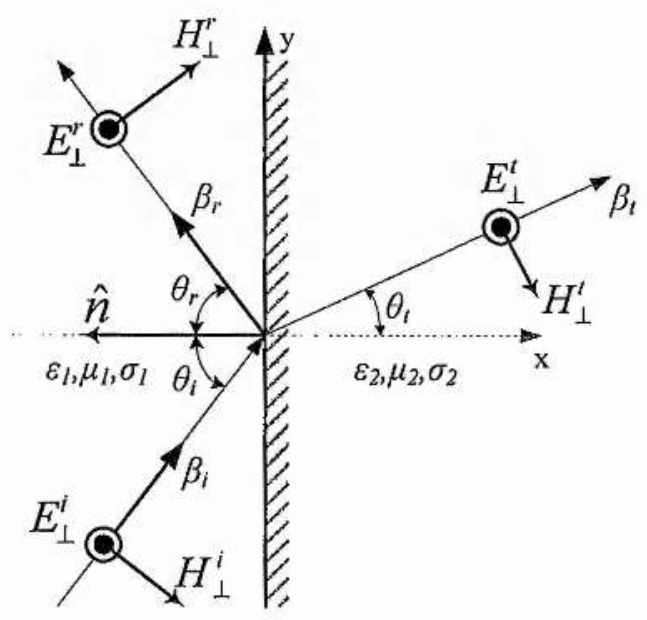

(a) Perpendicular polarisation 


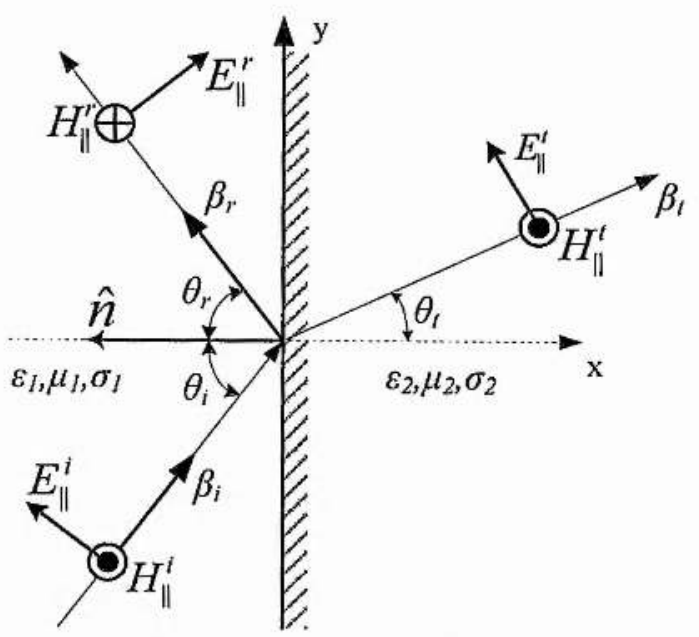

(b) Parallel polarisation

Figure 2-4: Boundary Model for both polarisations for an incident plane wave

For lossless media satisfying $(\sigma / \omega \varepsilon)^{2} \ll 1$, the Fresnel reflection coefficients can be found by [2]:

$$
\begin{gathered}
R_{\perp}=\frac{\sqrt{\frac{\mu_{2}}{\varepsilon_{2}}} \cos \left(\theta_{i}\right)-\sqrt{\frac{\mu_{1}}{\varepsilon_{1}}} \cos \left(\theta_{t}\right)}{\sqrt{\frac{\mu_{2}}{\varepsilon_{2}}} \cos \left(\theta_{i}\right)+\sqrt{\frac{\mu_{1}}{\varepsilon_{1}}} \cos \left(\theta_{t}\right)} \\
T_{\perp}=\frac{2 \sqrt{\frac{\mu_{1}}{\varepsilon_{1}}} \cos \left(\theta_{i}\right)}{\sqrt{\frac{\mu_{2}}{\varepsilon_{2}}} \cos \left(\theta_{i}\right)+\sqrt{\frac{\mu_{1}}{\varepsilon_{1}}} \cos \left(\theta_{t}\right)} \\
R_{\|}=\frac{-\sqrt{\frac{\mu_{1}}{\varepsilon_{1}}} \cos \left(\theta_{i}\right)+\sqrt{\frac{\mu_{2}}{\varepsilon_{2}}} \cos \left(\theta_{t}\right)}{\sqrt{\frac{\mu_{1}}{\varepsilon_{1}}} \cos \left(\theta_{i}\right)+\sqrt{\frac{\mu_{2}}{\varepsilon_{2}}} \cos \left(\theta_{t}\right)} \\
T_{\|}=\frac{2 \sqrt{\frac{\mu_{2}}{\varepsilon_{2}}} \cos \left(\theta_{i}\right)}{\sqrt{\frac{\mu_{1}}{\varepsilon_{1}}} \cos \left(\theta_{i}\right)+\sqrt{\frac{\mu_{2}}{\varepsilon_{2}}} \cos \left(\theta_{t}\right)}
\end{gathered}
$$


Since most dielectric materials have $\mu_{1}=\mu_{2}=\mu_{o}$ (excluding ferromagnetic ones) and using equations 2.19 and 2.20 , the Fresnel equations $2.22-2.25$ can be written as a function of $\theta_{i}$ :

$$
\begin{gathered}
R_{\perp}=\frac{\cos \left(\theta_{i}\right)-\sqrt{\frac{\varepsilon_{2}}{\varepsilon_{1}}} \sqrt{1-\frac{\varepsilon_{1}}{\varepsilon_{2}} \sin ^{2}\left(\theta_{i}\right)}}{\cos \left(\theta_{i}\right)+\sqrt{\frac{\varepsilon_{2}}{\varepsilon_{1}}} \sqrt{1-\frac{\varepsilon_{1}}{\varepsilon_{2}} \sin ^{2}\left(\theta_{i}\right)}} \\
T_{\perp}=\frac{2 \cos \left(\theta_{i}\right)}{\cos \left(\theta_{i}\right)+\sqrt{\frac{\varepsilon_{2}}{\varepsilon_{1}}} \sqrt{1-\frac{\varepsilon_{1}}{\varepsilon_{2}} \sin ^{2}\left(\theta_{i}\right)}} \\
R_{\|}=\frac{-\cos \left(\theta_{i}\right)+\sqrt{\frac{\varepsilon_{1}}{\varepsilon_{2}}} \sqrt{1-\frac{\varepsilon_{1}}{\varepsilon_{2}} \sin ^{2}\left(\theta_{i}\right)}}{\cos \left(\theta_{i}\right)+\sqrt{\frac{\varepsilon_{1}}{\varepsilon_{2}}} \sqrt{1-\frac{\varepsilon_{1}}{\varepsilon_{2}} \sin ^{2}\left(\theta_{i}\right)} \cos \left(\theta_{i}\right)} \\
\cos \left(\theta_{i}\right)+\sqrt{\frac{\varepsilon_{1}}{\varepsilon_{2}}} \sqrt{1-\frac{\varepsilon_{1}}{\varepsilon_{2}} \sin ^{2}\left(\theta_{i}\right)}
\end{gathered}
$$

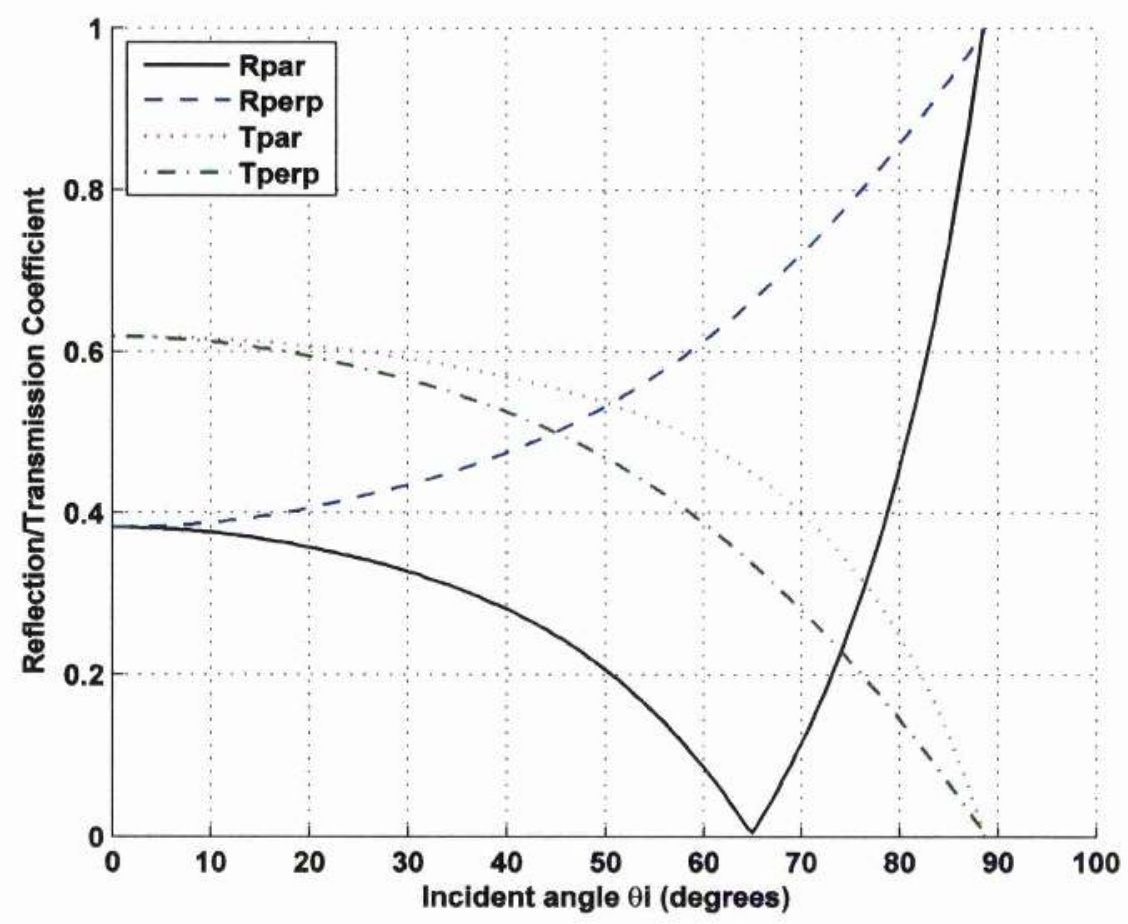

Figure 2-5: Fresnel Reflection and Transmission coefficients for a plane wave incident on a half space dielectric medium with relative permittivity $\varepsilon_{2}=3$

Figure 2-5 shows the magnitude of the Fresnel coefficients for a wave propagating in free space and impinging onto a dielectric half-space with relative permittivity $(\varepsilon=3)$ for both polarisations. 
It can be observed that total reflection occurs at $\theta=90^{\circ}$ for both polarisations. This situation is known as grazing incidence and is the case where the incident wave is tangential to the interface. Zero reflection occurs for parallel polarisation at one incident angle, which is known as 'Brewster's angle' and can be calculated from:

$$
\theta_{B}=\tan ^{-1}\left(\sqrt{\frac{\varepsilon_{2}}{\varepsilon_{1}}}\right)
$$

As already mentioned all the above formulation (2.22-2.29) apply for lossless media. However, the same formulation can be used for lossy media, where $(\sigma / \omega \varepsilon)^{2} \gg 1$, if $\varepsilon$ is replaced by its complex value $\varepsilon_{\text {complex }}$ of equation (2.13).

\subsubsection{Layer model (single slab model)}

The layer model provides more realistic results than the boundary one because it can take into consideration the thickness of the plane and is very well suited for deterministic radio propagation modelling. The single layer model (air-medium-air) is going to be presented here for simplicity.

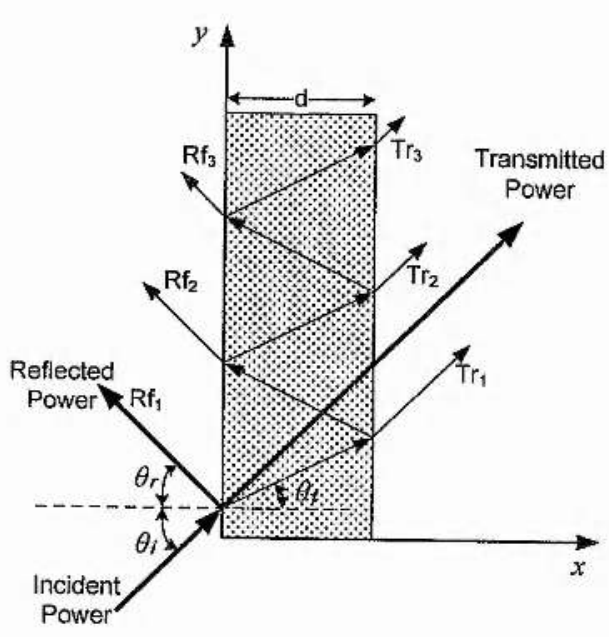

$2.6 \mathrm{a}$

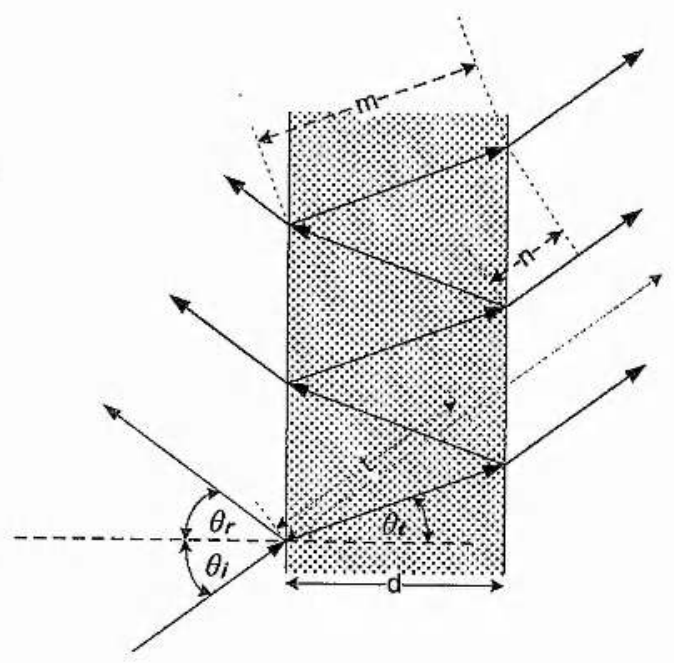

$2.6 \mathrm{~b}$

Figure 2-6: (a) Single layer model (b) Difference in ray paths lengths for various rays emerging from the layer

As it can be observed from Figure 2-6, multiple interactions exist between the incident ray and the two interfaces of the obstruction. In order to develop a solution for reflection and transmission coefficients the following definitions are made [6]: $R_{f}$ and $R_{2}$ are the interior and 
exterior to the layer reflection coefficients (based on the boundary model in section 2.4.1.1). Similarly $T_{\text {ext }}$ and $T_{i m t}$ are the exterior and interior transmission coefficients. Also $P_{d}$ is the phase delay of the field in a single crossing of the layer and $P_{a}$ is the phase difference between adjacent rays leaving the layer. These two can be calculated as:

$$
\begin{gathered}
P_{d}=e^{-\gamma r m} \\
P_{a}=e^{-\gamma 2 m \sin \left(\theta_{t}\right) \sin \left(\theta_{t}\right)}
\end{gathered}
$$

Where $\gamma^{\prime}$ and $\gamma$ are the propagation constants (Eq. 2.6) in the dielectric medium and in free space respectively while, $m$ is the distance covered by each ray for a single crossing inside the layer. Assuming an infinite dielectric slab (projects to infinity along the $+y$ and $-y$ axis as shown in Figure 2-6) the total transmission coefficient considering the internal reflections inside the slab can be written as:

$$
\begin{gathered}
T=T_{1}+T_{2}+T r_{3}+\cdots+T r_{n} \\
T=T_{1} T_{2} P_{a}+T_{1} T_{2} P_{a}^{3} P_{d} R_{2}^{2}+T_{1} T_{2} P_{a}^{5} P_{d}^{2} R_{2}^{4}+\cdots+T_{1} T_{2} P_{a}^{2(n-1)+1} P_{d}^{n-1} R_{2}^{2 n} \\
T=\frac{T_{1} T_{2} \sum_{i=1}^{n}\left(P_{a}^{2 i} P_{d}^{i-1} R_{2}^{2 i}\right)}{P_{a} R_{2}^{2}}
\end{gathered}
$$
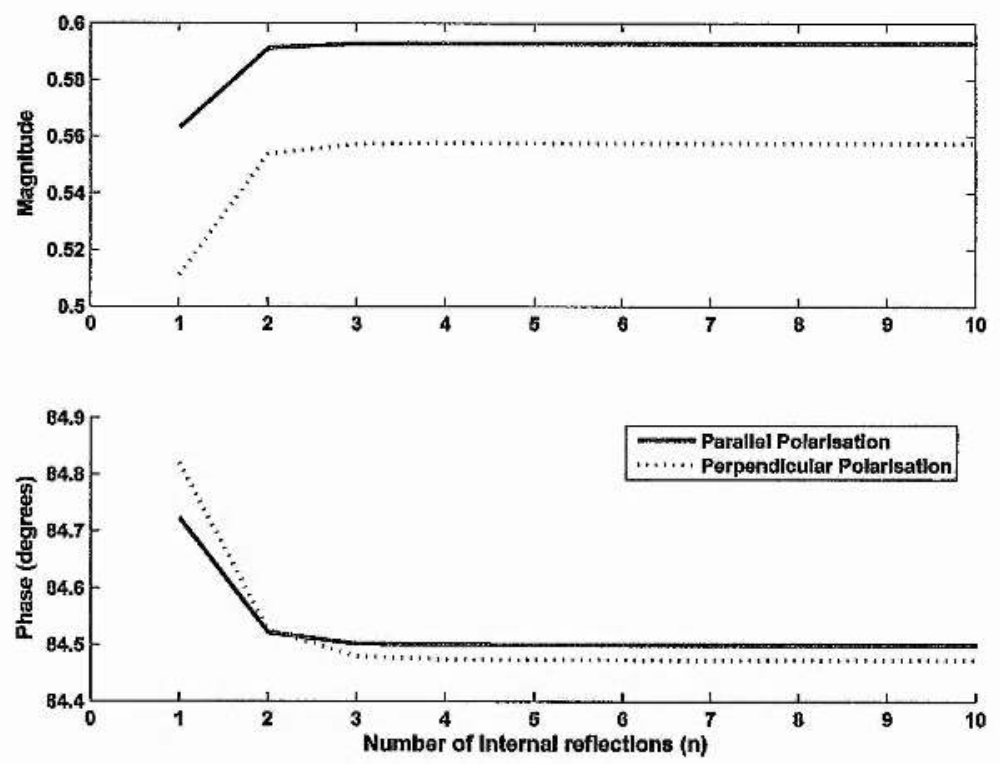

Figure 2-7: Magnitude and Phase of the Transmission coefficient as a function of the internal to the slab number of reflections for both polarisations $\left(\theta_{i}=30^{\circ}\right)$ 
Solving equation (2.33) for both perpendicular and parallel polarisations (using eqs. 2.26 - 2.29), it is observed that the complex transmission coefficients converge to a fixed value for large number of internal reflections $n$. This is illustrated in Figure 2-7. This happens because contributions from high order reflections inside the slab diminish and do not affect the overall sum. By recognising the geometrical series in (eq. 2.33), for $n$ going to infinity, and replacing $R^{\prime}=R_{2}=-R 1$ and $T=1-R$, it can be proven that $[5]$ :

$$
T=\frac{\left(1-R^{\prime 2}\right) e^{-j\left(\delta-K_{o} d\right)}}{1-R^{\prime 2} e^{-j 2 \delta}}
$$

Similarly the overall reflection coefficient can be proven to be,

$$
R=\frac{1-e^{-j 2 \delta}}{1-R^{\prime 2} e^{-j 2 \delta}} R^{\prime}
$$

where $\delta=\frac{2 \pi d}{\lambda} \sqrt{\varepsilon_{\text {complex }}-\sin ^{2}\left(\theta_{i}\right)}, k_{o}=2 \pi / \lambda, \lambda$ is the free space wavelength, and $\varepsilon_{\text {complex }}$ is the complex relative permittivity given by (eq.2.13) and $d$ is the thickness. $R^{\prime}$ is either replaced with $R_{\perp}$ (eq 2.26) or $R_{\|}$(eq 2.28) to account for perpendicular or parallel polarisations respectively.

\subsubsection{Multilayer Model}

It is sometimes the case that the transverse walls/interfaces consist of more than one layer of dielectric mediums (e.g. double glazed windows). In these cases a multi-layer model needs to be utilised. Such a model is briefly presented here but much more information and more detailed formulations can be found in [7] and [8]. Figure 2-8 shows a multilayer dielectric structure of $n$ layers, illustrating the forward and reverse propagating waves ( $c_{i}$ and $b_{i}$ respectively). By utilising the wave-chain-matrix theory [9] the solution to this problem takes the following form,

$$
\left[\begin{array}{l}
c_{1} \\
b_{1}
\end{array}\right]=\prod_{i=1}^{n} \frac{1}{T_{i} T_{n+1}}\left[\begin{array}{cc}
e^{j \gamma_{i} d_{i}} & R_{i} e^{-j \gamma_{i} d_{i}} \\
R_{i} e^{j \gamma_{i} d_{i}} & e^{-j \gamma_{i} d_{i}}
\end{array}\right]\left[\begin{array}{cc}
1 & R_{n+1} \\
R_{n+1} & 1
\end{array}\right]\left[\begin{array}{l}
c_{n+1} \\
b_{n+1}
\end{array}\right]
$$

where $d_{i}(\mathrm{~m})$ is the thickness and $\gamma_{i}$ is the propagation constant of the $i^{\text {th }}$ layer (see eq. 2.6). The Fresnel reflection and transmission coefficients of the $i^{\text {th }}$ layer $\left(R_{i}\right.$ and $\left.T_{i}\right)$ are derived from the layers' wave impedances $Z_{i}$ as:

$$
R_{i}=\frac{Z_{i}-Z_{i-1}}{Z_{i}+Z_{i-1}} \text { and } T_{i}=1-R_{i}
$$




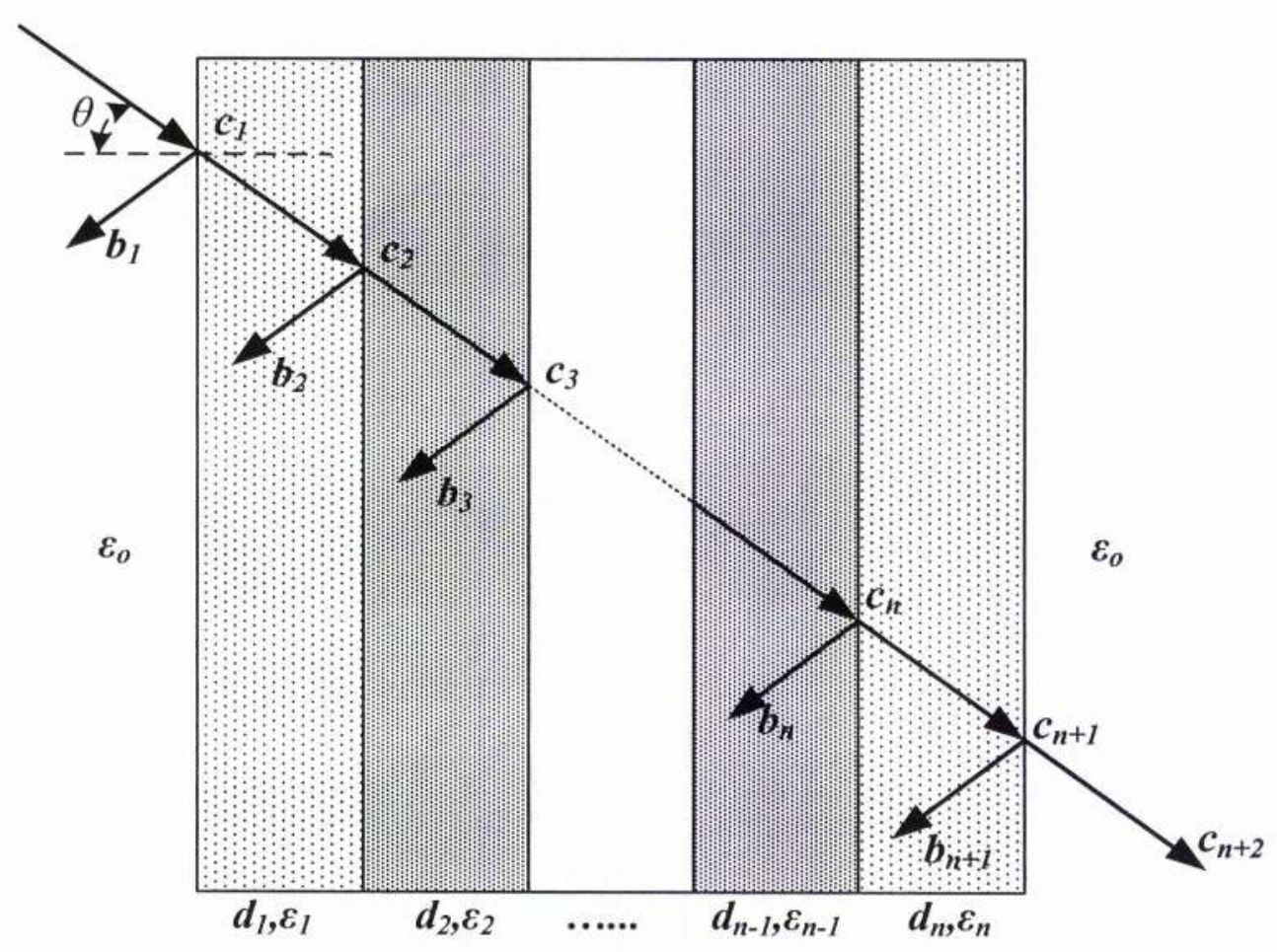

Figure 2-8: Boundary value solution for an n-layer dielectric wall

To account for the incident wave polarisation $Z_{i}$ is either defined for parallel or perpendicular polarisation as,

$$
\begin{aligned}
Z_{i}^{\text {perp }} & =\frac{\cos \theta}{\sqrt{\varepsilon_{r i}-\sin ^{2} \theta}} \\
Z_{i}^{\text {par }} & =\frac{\sqrt{\varepsilon_{r i}-\sin ^{2} \theta}}{\varepsilon_{r i} \cos \theta}
\end{aligned}
$$

where $\theta$ is the angle of incidence and $\varepsilon_{r i}$ is the complex relative permittivity (eq. 2.13) of the $i^{\text {th }}$ layer.

After carrying out the matrix multiplications in (eq. 2.36), it can be shown that,

$$
\left[\begin{array}{l}
c_{1} \\
b_{1}
\end{array}\right]=\left[\begin{array}{ll}
A_{11} & A_{12} \\
A_{21} & A_{21}
\end{array}\right]\left[\begin{array}{l}
c_{n+2} \\
b_{n+2}
\end{array}\right]
$$

The total reflection and transmission coefficients are then given by

$$
\begin{gathered}
R=\frac{b_{1}}{c_{1}}=\frac{A_{21}}{A_{11}} \\
T=\frac{c_{n+2}}{c_{1}}=\frac{1}{A_{11}}
\end{gathered}
$$




\subsubsection{Ray Fixed Coordinate System}

The formulation for reflection and transmission presented so far are valid for linear polarisations. For circular or elliptical polarisation the incident plane wave can be represented as a vector sum of two waves having orthogonal linear polarisations. Therefore the reflected wave will also consist of two orthogonal waves with perpendicular and parallel polarisation and their fields can be obtained as follows [4],

$$
\left[\begin{array}{l}
E_{\|}^{r} \\
E_{\perp}^{r}
\end{array}\right]=\left[\begin{array}{cc}
R_{\|} & 0 \\
0 & R_{\perp}
\end{array}\right]\left[\begin{array}{l}
E_{\| 1}^{i} \\
E_{\perp}^{i}
\end{array}\right]
$$

where $E_{r \|}$ and $E_{r \perp}$ are the reflected field components for parallel and perpendicular polarisation. Likewise $E_{i \|}$ and $E_{i \perp}$ are the incident field components for parallel and perpendicular polarisation respectively. Similarly the total transmitted field is given by,

$$
\left[\begin{array}{l}
E_{\| !}^{t} \\
E_{\perp}^{t}
\end{array}\right]=\left[\begin{array}{cc}
T_{\|} & 0 \\
0 & T_{\perp}
\end{array}\right]\left[\begin{array}{l}
E_{\| !}^{i} \\
E_{\perp}^{i}
\end{array}\right]
$$

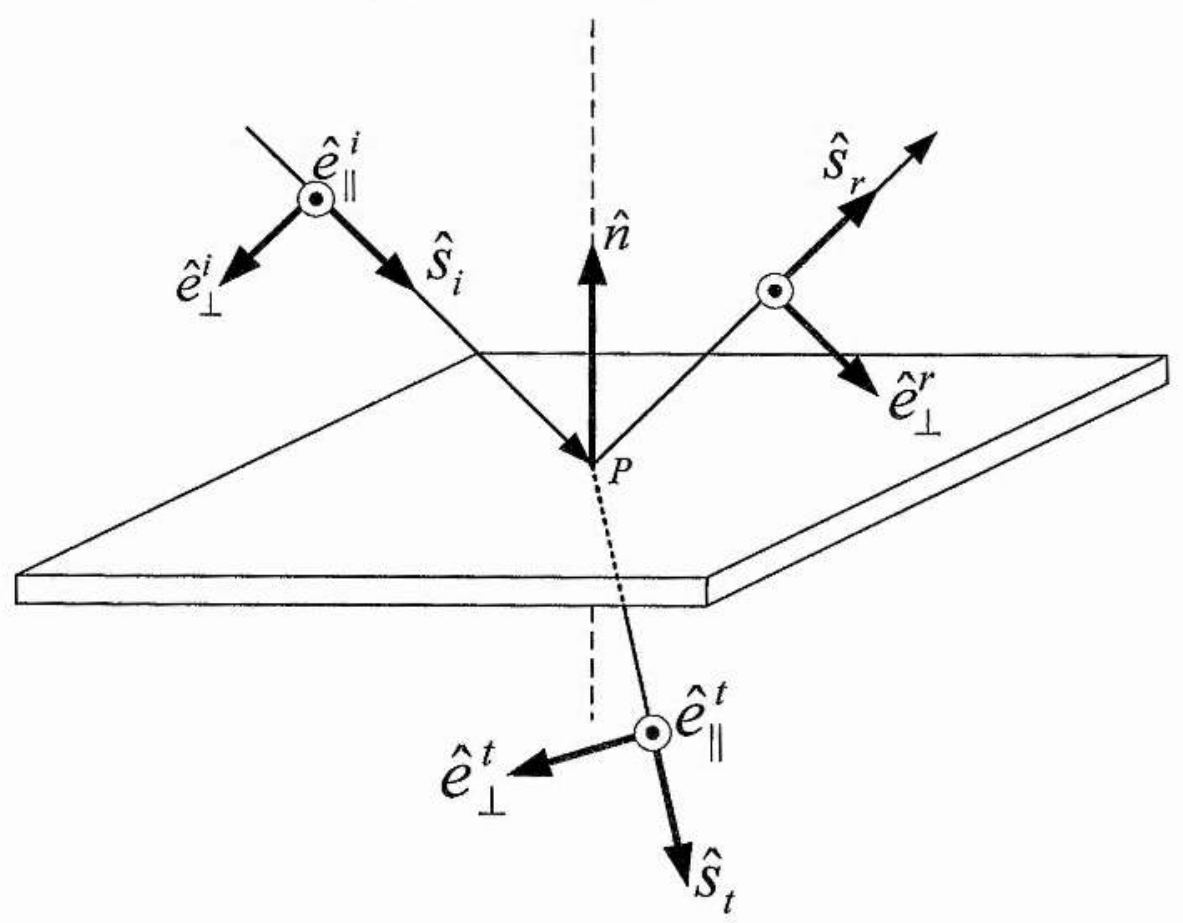

Figure 2-9: Three Dimensional Reflection and Transmission

The system presented here is the local ray-fixed coordinate system since it is inextricably linked with the ray directions $\hat{s}_{i}, \hat{s}_{r}$, and $\hat{n}$ which are all unit vectors as shown in Figure 2-9. This, can be written as follows [9] [10], 


$$
\begin{gathered}
\hat{e}_{\|}^{i}=\frac{\hat{s}_{i} \times\left(\hat{n} \times \hat{s}_{i}\right)}{\left|\hat{s}_{i} \times\left(\hat{n} \times \hat{s}_{i}\right)\right|}, \quad \hat{e}_{\perp}^{i}\left(\hat{n} \cdot \hat{s}_{i}\right)=\hat{n} \times \hat{e}_{\|}^{i} \\
\hat{e}_{\|}^{r}=\frac{\hat{s}_{r} \times\left(\hat{n} \times \hat{s}_{r}\right)}{\left|\hat{s}_{r} \times\left(\hat{n} \times \hat{s}_{r}\right)\right|}, \hat{e}_{\perp}^{r}\left(\hat{n} \cdot \hat{s}_{r}\right)=\hat{n} \times \hat{e}_{\|}^{r} \\
\hat{e}_{\|}^{t}=\frac{\hat{s}_{t} \times\left(\hat{n} \times \hat{s}_{t}\right)}{\left|\hat{s}_{t} \times\left(\hat{n} \times \hat{s}_{t}\right)\right|}, \hat{e}_{\perp}^{t}\left(\hat{n} \cdot \hat{s}_{t}\right)=\hat{n} \times \hat{e}_{\|}^{t} \\
E_{\|}^{i}=\hat{e}_{\|}^{i} E^{i}, E_{\perp}^{i}=\hat{e}_{\perp}^{i} E^{i} \\
E^{r}=\hat{e}_{\|}^{r} E_{\|}^{r}+\hat{e}_{\perp}^{r} E_{\perp}^{r}=\hat{e}_{\|}^{r} R_{\|} \hat{e}_{\|}^{i} E^{i}+\hat{e}_{\perp}^{r} R_{\perp} \hat{e}_{\perp}^{i} E^{i}=\overline{\bar{R}} E^{i}
\end{gathered}
$$

where $\overline{\bar{R}}$ is the dyadic reflection coefficient given by:

$$
\overline{\bar{R}}=\hat{e}_{\|}^{r} \hat{e}_{\|}^{i} R_{\|}+\hat{e}_{\perp}^{r} \hat{e}_{\perp}^{i} R_{\perp}
$$

Similarly, it can be proven that,

$$
\overline{\bar{T}}=\hat{e}_{\| \mid}^{r} \hat{e}_{\|}^{i} T_{\|}+\hat{e}_{\perp}^{r} \hat{e}_{\perp}^{i} T_{\perp}
$$

where $R_{\|}, R_{\perp}, T_{\|}$and $T_{\perp}$ can be obtained using any of the aforementioned models (boundary, single or multi-layer).

In the 3D Cartesian system the reflected and transmitted electric fields at point $\mathrm{P}$ can be calculated as follows:

$$
\begin{aligned}
& \overrightarrow{\vec{E}}_{P}^{r}(x, y, z)=\overline{\bar{R}} \overrightarrow{\vec{E}}_{P}^{i}(x, y, z) \\
& \overrightarrow{\vec{E}}_{P}^{t}(x, y, z)=\overline{\bar{T}} \overrightarrow{\vec{E}}_{P}^{i}(x, y, z)
\end{aligned}
$$

Now it becomes obvious from equations 2.52 and 2.53 that the incident electric field needs to be decomposed into $x, y$ and $z$ components.

\subsubsection{Electric Field decomposition}

In order to decompose the incident electric field into $x, y$ and $z$ components the antenna radiation pattern need to be taken into account. The incident electric field can be then written as [11],

$$
\vec{E}(x, y, z)=E_{o} P(\theta) \frac{e^{-j k r}}{\sqrt{4 \pi} r} \hat{\theta}
$$

where

$$
E_{o}=\sqrt{2 Z_{o} P_{o} G_{T}}
$$


$Z_{o}$ is the free space wave impedance, $P_{o}$ is the transmitted power, and $G_{T}$ is the transmitter gain. Also $r$ is the distance travelled by the wave and $P(\theta)$ is the antenna radiation pattern. For a vertical dipole this is $P(\theta)=\sin (\theta) . \hat{\theta}$ is a unit vector which can be determined by [11],

$$
\left[\begin{array}{l}
\hat{r} \\
\hat{\theta} \\
\hat{\varphi}
\end{array}\right]=\left[\begin{array}{ccc}
\sin (\theta) \cos (\varphi) & \sin (\theta) \sin (\varphi) & \cos (\theta) \\
\cos (\theta) \cos (\varphi) & \cos (\theta) \sin (\varphi) & -\sin (\theta) \\
-\sin (\varphi) & \cos (\varphi) & 0
\end{array}\right]\left[\begin{array}{l}
\hat{x} \\
\hat{y} \\
\hat{z}
\end{array}\right]
$$

where $\theta$ and $\varphi$ are the elevation and azimuth angles respectively in the antenna radiation pattern as shown in Figure 2-10 (the antenna is located in the centre of the coordinate system).

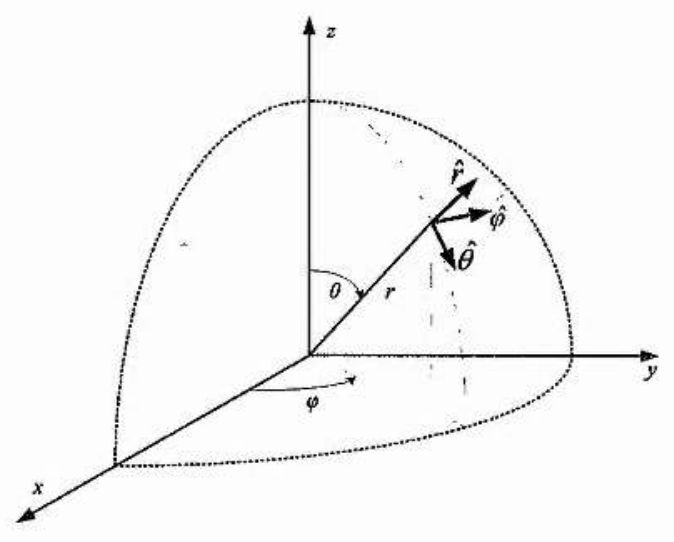

Figure 2-10: Coordinate System for antenna calculations

\subsubsection{Scattering}

The reflection mechanism discussed so far, assumed that the surface is smooth (specular reflection). In cases where the surface becomes rougher, the incident ray will be scattered from a large number of positions on the surface, which means that too many rays will follow arbitrary directions. This broadens the scattered energy as shown in Figure 2-11, which effectively means that the energy in the specular direction is reduced. The degree of scattering depends on the incidence angle and on the roughness of the surface, compared to the wavelength of transmission. The surface can be considered smooth when the phase difference between different waves reflected from the surfaces are less than $90^{\circ}$. This can be written as,

$$
\Delta \phi=\frac{4 \pi \Delta h \cos \theta_{i}}{\lambda}<90^{\circ}
$$

which leads to the Rayleigh criterion that states that a smooth surface is the one that $\Delta h$ is

$$
\Delta h<\frac{\lambda}{8 \cos \theta_{i}}
$$



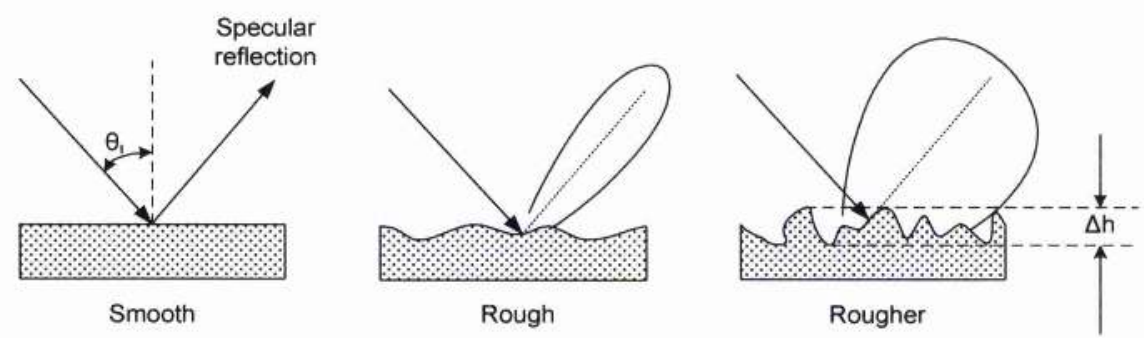

Figure 2-11: Rough surface scattering

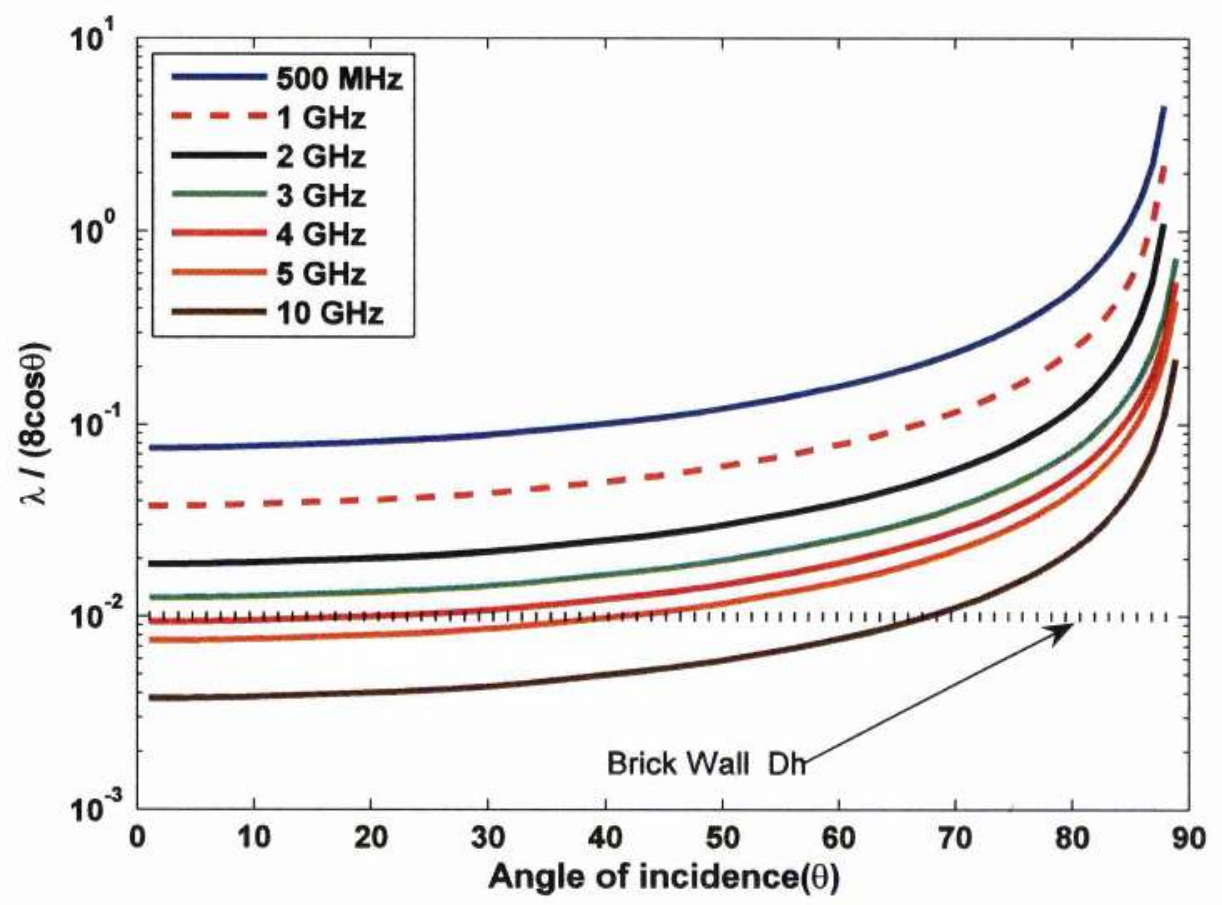

Figure 2-12: Rayleigh Criterion for surface roughness

Figure 2-12 illustrates an implementation of the Rayleigh criterion (eq. 2-58). It is observed that any surface is susceptible to scattering for smaller angles of incidence and/or for higher frequencies. A typical UK brick (CCSR external wall) wall has $\Delta h=1 \mathrm{~cm}$ (obtained by measuring the step that the brick makes with the dried mortar). As shown in the figure, up to 4 $\mathrm{GHz}$, the surface can be considered smooth, but for higher frequencies and small angles of incidence the surface becomes rough. This means that WLAN 802.11a and WiMAX systems would suffer scattering from typical UK brick Walls.

To account for scattering, the specular reflection coefficient $R$ needs to be multiplied by a roughness factor $f\left(\sigma_{s}\right)$, which is less than unity and depends exponentially on the standard deviation of the surface height $\sigma_{s}$ and the angle of incidence $\theta_{i}$. This is given by 


$$
f\left(\sigma_{s}\right)=e^{-\frac{1}{2}\left(\frac{4 \sigma_{s} \cos \theta_{i}}{\lambda}\right)}
$$

However, this factor multiplication accounts only for the field reduction along the specular direction and does not consider the scattered components. The rest of the energy that is not reflected to the specular direction is carried by non-specular propagating modes which begin to propagate at higher frequencies. It is often the case that significant amount of the propagating energy is carried by a non-specular reflection. In such cases, conventional Ray Tracing techniques (based on Geometric Optics) would fail to provide accurate predictions. Surface roughness cannot be modelled using Ray-Tracing techniques since one of the basic GO assumptions is that surfaces are large compared to the wavelength. For such cases, Ray Tracing can be enhanced by full-wave electromagnetic techniques to account for rough surface scattering.

\subsubsection{Diffraction}

During the discussion of the reflection and transmission propagation mechanisms it has been assumed that there is an infinitely sharp transition between the shadow and the illumination regions. If this is the case then no energy propagates into the shadow regions. However, in practice transitions are never infinitely sharp and as a consequence of this some energy does propagate into the shadow region.

This propagation mechanism is called Diffraction and can be described by Huygen's principle [4], illustrated in Figure 2-13, which states that:

- Every point at the advancing wavefront of the primary wave can be considered as a source of secondary spherical wavelets which have the same frequency and velocity as the primary wave.

- The position of the wavefront at any later time is the envelope of all such wavelets.

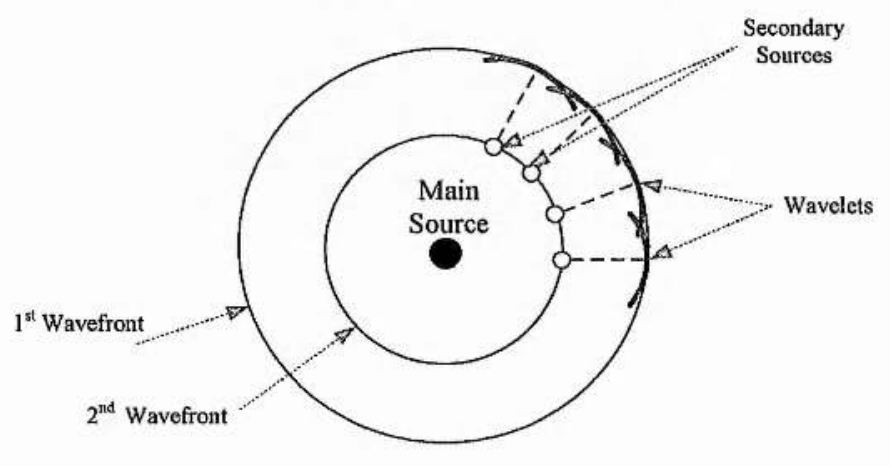

Figure 2-13: Huygen's Principle

Huygen's principle can be used to predict diffraction of a plane wave over an absorbing plane, or knife edge. Figure 2-14 shows how the diffracted field propagates into the shadow region 
when applying Huygens's principle to a plane wave incident on a knife-edge. The incident plane wavefronts impinge on the edge from the left and become curved by the edge, so that wave propagates into the shadowed region. Mathematically, the contributions from an infinite number of secondary sources in the region above the edge are summed with respect to their amplitude and phase in order to calculate the diffracted field.

Knife-edge diffraction can be also considered in terms of the Fresnel Zone obstruction around the direct ray. Fresnel zones are formed by a number of concentric ellipsoids around the direct path and can be thought of as containing the propagated energy in the wave. The $n^{\text {th }}$ Fresnel zone is the region inside an ellipsoid defined by the locus of points where the distance is larger than the direct path between transmitter and receiver by $n$ half-wavelength [4]. Contributions within the first zone are all in phase, so any obstructions, which do not enter this zone, will have little effect on the received signal [13]. When $60 \%$ of the first Fresnel zone is kept clear of obstructions, then the total path attenuation will be practically the same as in the unobstructed case. Details on the Fresnel zone concept, the single knife-edge diffraction approach and its extension to the multiple knife-edge approaches, can be found in [12], [13].

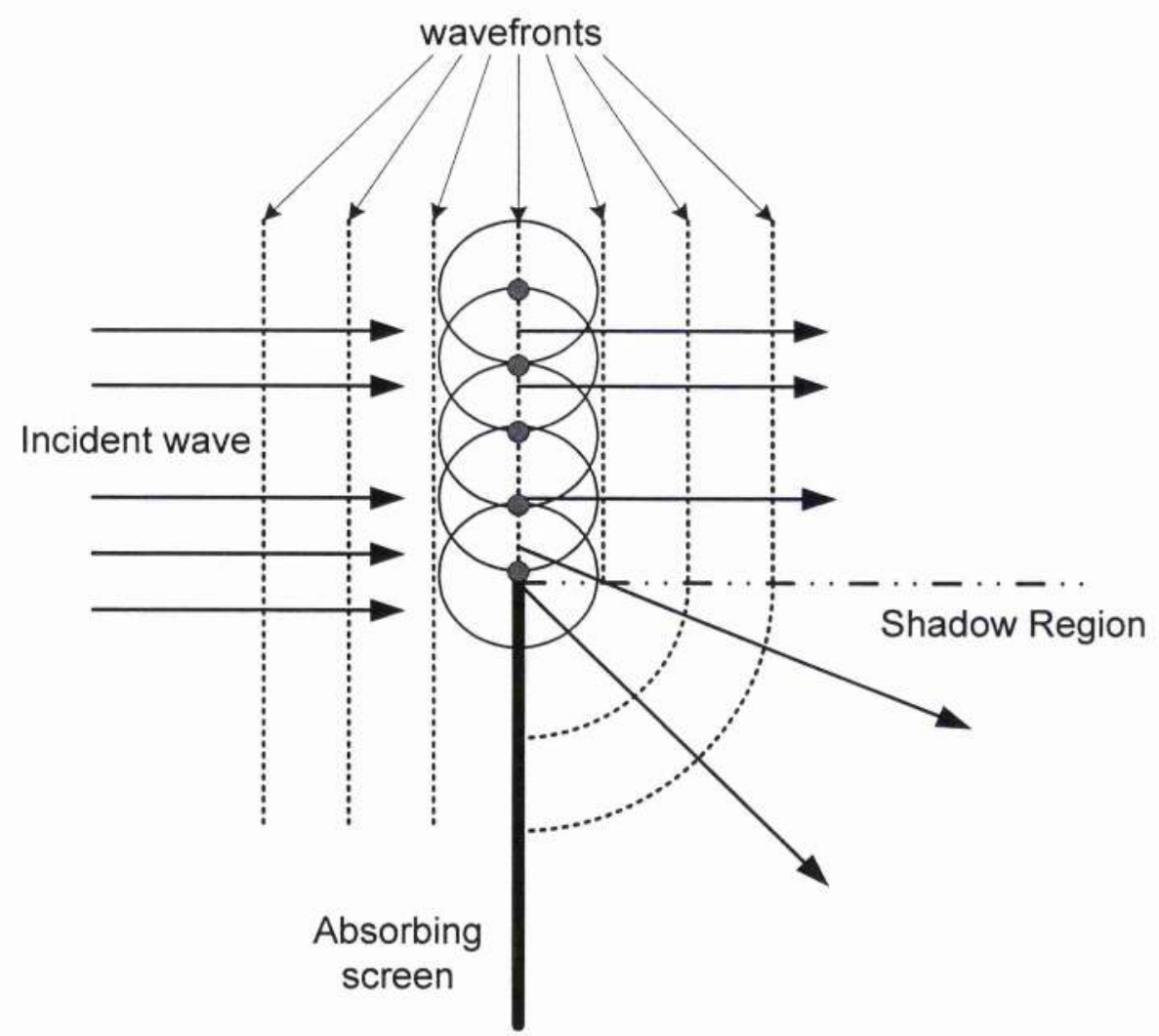

Figure 2-14: Huygen's Principle for knife-edge diffraction 
It is usually the case in deterministic radio propagation modelling, that it is necessary to model the exact shape and the morphological description of the obstructions. This can be done using the Geometrical Theory of Diffraction (GTD) and its extension the Uniform Theory of Diffraction. Details and formulation of these approaches will be presented later on this thesis. (see section 3.2)

\subsection{Radio Propagation modelling}

Radio propagation modelling is a well established technique for analysing radio coverage in a wireless scenario by predicting the received field (or power) at all the possible receiver locations. Propagation models are classified into three categories based on the way they calculate path loss or the received signal strength. These are empirical, semi-empirical and deterministic (or site-specific) models. This section presents these three categories and describes their potential advantages and disadvantages.

\subsubsection{Path loss}

Path loss is an essential information for determining the radio coverage of a transmitting antenna (Base Station or access point) [32]. It is defined as the ratio of the received power $P_{r}$ to the transmitted power $P_{t}$, usually expressed in $\mathrm{dB}[3]$.

$$
L(d B)=10 \log \left(\frac{P_{r}}{P_{t}}\right)
$$

In free space, where there is a Line of Sight (LOS) between the transmitting and receiving antennas, the path loss is considered to follow an inverse square law with respect to the distance travelled by the waves [14]. It also depends on the frequency of transmission. Therefore the free space loss can be written as:

$$
L_{\text {free }}(d B)=10 \log \left(\frac{G_{t} G_{r} \lambda^{2}}{(4 \pi)^{2} R^{2}}\right)
$$

Where $G_{t}$ and $G_{r}$ are the gains of the transmitter and the receiver antennas respectively, $\lambda$ is the wavelength and $R$ is the distance away from the transmitter. For $G_{t}$ and $G_{r}$ equal to unity the above equation can also be written as [4],

$$
L_{\text {free }}(d B)=32.4+20 \log R_{K m}+20 \log F_{M H z}
$$

which clearly shows that by doubling either the distance or the frequency, the free space loss increases by $6 \mathrm{~dB}$ (inverse square law). 


\subsubsection{Empirical and Semi-Empirical Models}

Empirical Models are described by a set of equations fitted to the results obtained from extensive number of path loss measurements [4][11]. They are simple and efficient (fast) to use since the estimation is usually calculated from this set of equations. They are relatively accurate for environments which have the same characteristics as the one where the measurements were originally performed but error standard deviation tends to be large. The input parameters for such models are usually qualitative and not so specific. Their main disadvantage is that they are not applicable for different environments without modifications and may be considered as nonapplicable for indoor environments [32] due to their inability to provide accurate results for disparate scenarios (in other words they are hard to generalise). They do not provide any physical insight into the propagation mechanisms discussed in section 2.4 and this could lead to wrong predictions when the environmental clutter becomes increasingly complex (indoor environments).

Semi-Empirical Models are based on equations derived from partly the application of deterministic methods [11]. These equations are based on the characteristics surrounding the transmitter and the environmental characteristics of a particular scenario. They require more detailed information about the environment than empirical models but less than deterministic ones. Again, as for empirical models, it is hard to use these kinds of models for different scenarios.

Several Empirical and Semi-Empirical Models have been proposed in literature. These models are more or less environment and frequency range specific. Some of the better known ones are:

- Okumura-Hata Model [15]. This is a fully empirical model, which is based on an extensive series of measurements carried out in Tokyo city between $150 \mathrm{MHz}-1.5 \mathrm{GHz}$. The predictions are made through a series of graphs, the most important of which have been approximated by Hata [16]. These model can be used for open, urban and suburban area predictions.

- Ibrahim and Parsons Model [17] is intended as a first step in quantifying urban propagation loss. It is based upon measurements made around London, UK.

- Allsebrook and Parsons Model [18] is a semi-empirical model for suburban predictions. It is based on measurements made in three British cities (Bradford, Bath and Birmingham) at 86,167 and $441 \mathrm{MHz}$.

- Ikegami Model [19] is a semi-deterministic model which attempts to produce deterministic predictions at specific points. It uses a detailed description of the environment and calculates only reflections from the surrounding clutter with the 
reflection loss being constant at a fixed value. Diffraction is calculated using single edge approximation.

- Walfish-Bertoni Model [20] is a semi-deterministic model which can be used to predict multiple diffraction over building rooftops.

- Cost-231-Hata model [21] is an extension of the Okumura-Hata Model to cover the 1500$2000 \mathrm{MHz}$ band.

- Lee Model [22] is an empirical power law model with path loss exponents derived from measurements at $900 \mathrm{MHz}$. It can be also applied for other frequencies if correction factors are used.

All the above models deal with outdoor propagation predictions. In fact, empirical and semiempirical models exist for indoor or outdoor-indoor propagation. COST231 LOS and NLOS [4], [21] models can be used for predicting indoor radio propagation in floors and between floors, and ITU-R P.1238-2 can be used for predicting indoor radio communication in the frequency range $900 \mathrm{MHz}-100 \mathrm{GHz}$. Nevertheless, for increased accuracy, deterministic or site-specific models should be employed.

\subsubsection{Deterministic or Site-Specific Models}

Deterministic or Site-specific Models are based on the application of well-known electromagnetic techniques and numerical methods (such as Ray Tracing) to a site-specific environmental description which is obtained from the particular environment (building) database [11] or the finite-difference-time-domain (FDTD) method. The main advantage of these models is that they are generic for arbitrary environments. For a given environmental description they use electromagnetic theory to estimate the field strength at every possible receiver location. They provide the ability to the engineer to define the two-dimensional or three-dimensional geometry of the environment by means of facets specifying the location of the buildings' walls, the electrical properties of these walls (constitutive parameters such as permittivity and conductivity), the transmitter location, the antenna patterns and polarisations and the frequency of transmission. Considering all these properties of deterministic models, it can be concluded that they are very well suited for indoor environments since they can provide relatively highly accurate predictions. The main drawback of this way of modelling is the large computational overhead required which effectively means extremely high computational power is required (sufficient memory and fast processing). 


\subsection{Summary}

The basic electromagnetic theory which establishes the required key parameters and equations for determining the electric field through radio propagation modelling has been presented in this chapter. This has included the fundamental Maxwell's equations, their plane wave solution and the various radio propagation mechanisms which define the interaction of radio waves with various environment obstructions. Parameters, such as polarisation and constitutive parameters which are essential into the calculation of the various radio propagating mechanisms have also been presented.

This chapter briefly presented radio propagation modelling by classifying the various approaches into three categories; empirical, semi-empirical and deterministic modelling. The potential advantages and disadvantages of these three categories have been outlined. It has been identified that when accuracy is preferred over simulation time and computational load deterministic modelling is the preferred category. The next chapter presents in detail this deterministic modelling approach through the use of Ray Tracing and Geometric Optics (GO). 


\section{Chapter 3}

\section{Deterministic Modelling using Ray Tracing}

Ray Tracing is the dominant deterministic technique used to predict propagation effects in mobile and personal communication environments. It is based on Geometrical Optics (GO), which is an easily applied approximate method for predicting a high-frequency electromagnetic field. Ray Tracing is used to identify all the possible ray paths between the transmitter and possible receiver locations and uses high-frequency electromagnetic theory to calculate the amplitude, phase, delay and polarisation of each ray. Two main algorithms exist for the implementation of Ray-Tracing; the ray launching and the image method.

This chapter presents the basic principles and algorithms of Ray Tracing, and the high frequency methods used to reduce the complexity of the electromagnetic problem to be solved (Geometrical Optics GO and its extensions: Geometrical theory of Diffraction GTD and Uniform Theory of Diffraction UTD).

\subsection{Geometric Optics (GO)}

As already mentioned, Geometric Optics represent a simple way for predicting approximately the electric field at any possible receiver location. It assumes an infinite frequency for the propagating signal in such as way as to consider all the propagating energy to be contained inside very thin tubes, called rays [24]. Using this theory the reflected and transmitted fields can be determined. However, this theory is subject to the following assumptions [4]:

- Waves are locally plane to the points of interaction

- Wavelength is small compared to the distance between the source and the first interactions along each ray path and the distance between individual interactions.

- Surfaces are large compared to the wavelength of transmission

- Curvature of Surfaces is small compared to the wavelength.

In GO the electric field is given by [11],

$$
E(s)=E(0) \sqrt{\frac{\rho_{1} \rho_{2}}{\left(\rho_{1}+s\right)\left(\rho_{2}+s\right)}} \mathrm{e}^{-\mathrm{jks}}
$$


which is the $\mathrm{GO}$ electric field at a point, at a distance $s$ away from the reference point $s=0 . E(0)$ is the electric field at the reference point. $k$ is the wavenumber of the medium (free space). $\rho_{1}, \rho_{2}$ are the principal radii of curvature of the wavefront associated with the ray at the reference point which depends on the caustic lines as shown in Figure 3-1. The field at a caustic line is infinite, in principle, because an infinite number of rays pass through it. It is apparent that (3.1) becomes infinite when $\mathrm{s}=-\rho_{1}$ or $\mathrm{s}=-\rho_{2}$. Energy is spread over a bigger area across the second wavefront and hence, for the conservation of energy theorem to apply, the field strength across the second wavefront can be calculated from (3.1).

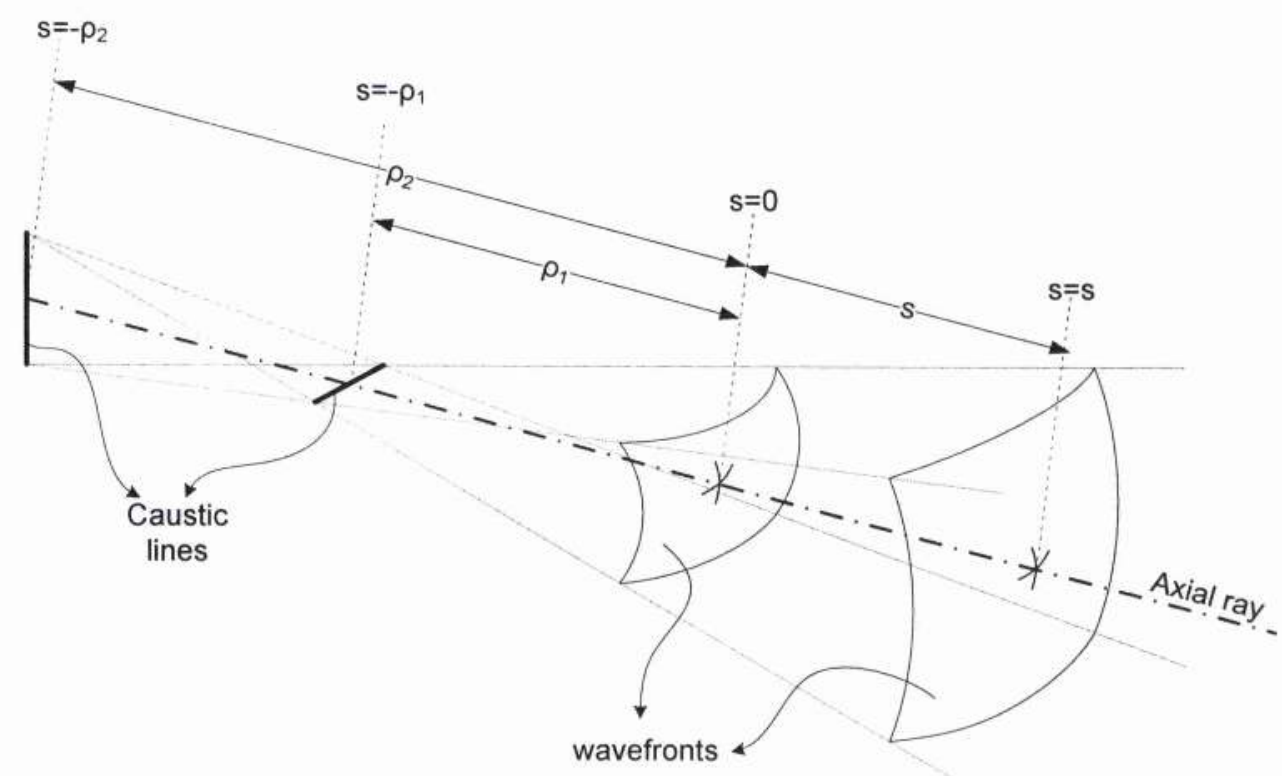

Figure 3-1: Ray propagating through two successive wavefronts

For spherical wavefronts both caustics degenerate at the same point (caustic or focal point), hence $\rho_{1}=\rho_{2}=\rho_{o}$ Therefore equation (3.1) becomes,

$$
E(s)=E(0) \frac{\rho_{o}}{\left(\rho_{o}+s\right)} e^{-j k s}
$$

, where $\rho_{o}$, is the radius of curvature in any direction. When the caustic (focal) point is taken as reference then equation (3.2) becomes,

$$
E(s)=E(0) \frac{1}{s} e^{-j k s}
$$

Similar formulation exists for cylindrical waves where one of the caustic lines approaches infinity [11]. 
In GO the free-space far-field electric field of a spherical wave at distance $r$ can be then approximated as,

$$
E(s)=E_{o} \frac{e^{-j k r}}{\sqrt{4 \pi} r}
$$

where $E_{o}$ is the transmitted electric field given by $E_{o}=2 Z_{o} P_{o} G_{T}$ (eq. 2.55). When the 3D antenna radiation pattern needs to be included equation (2.54) should be utilised.

The first step of GO is to calculate all the important ray paths (based on predefined criteria) between the transmitter and all the possible receiver locations, which are consistent with Snell's Laws of Reflection and Transmission. This step usually requires high computational costs in order to identify all the possible rays. For these reason only the rays that have the highest impact on the overall result are considered; these are usually the rays that suffer less interactions between the transmitter and the field points. Once the most important ray paths have been identified, electromagnetic theory is used to calculate the Fresnel's coefficients at each interaction point as if incident waves were plane and boundaries were plane and infinite [4]. Thirdly, the amplitude of each ray path should be corrected to account for the wavefront curvature from the source and the curvature of the boundaries. Finally all ray paths are summed up with regard to phase and amplitude. The following equation is used to formulate this procedure.

$$
E=\sum_{i} E_{o} A_{i}\left[\prod_{j=1}^{N_{R i}} R_{i, j} \prod_{m=1}^{N_{T i}} T_{i, m}\right] e^{-j k r_{i}}
$$

where $N_{R i}, N_{T i}$ are the number of reflections and transmissions of each ray path i respectively. $R_{i, j}$ and $T_{i, m}$ are the corresponding reflection and transmission coefficients. $\mathrm{r}_{\mathrm{i}}$ is the total path length and the factor $A_{i}$ accounts for the wavefront curvature as well as the curvature of the reflection and transmission boundaries (i.e. walls) also known as spreading factor. $E_{o}$ is the reference field at the transmitter. Finally the exponential term corresponds to the phase difference due to propagation delay of each ray. It needs to be noted that for grazing incidence (i.e. 900 degrees) the reflected component grazes the surfaces and GO will fail to provide a sufficient solution for the Reflection coefficient and therefore this case needs to be treated separately [10].

By utilising the Geometric Optics theory it becomes impossible to predict diffraction over obstructions because it ignores that energy does propagate into the shadowing region as seen in section 2.4.3. For this reason the method has included to the Geometrical Theory of Diffraction (GTD) in order to include diffraction. 


\subsection{GTD and UTD}

Geometrical Theory of Diffraction (GTD) which was developed by Keller [25], is based on Fermat's principle which is used to predict the existence of any diffracted rays. This theory is of particular importance in many cases and scenarios where diffraction might be the dominant propagation mechanism. It overcomes the GO inability to calculate the diffracted fields in the shadowed region. According to Keller, a ray obliquely incident upon the edge of an obstruction at an angle $\beta$ to the edge produces a cone (Keller cone) of diffracted rays having a semi-angle $\beta$. This is illustrated in Figure 3-2. When $\beta=90^{\circ}$ the cone becomes a disc.

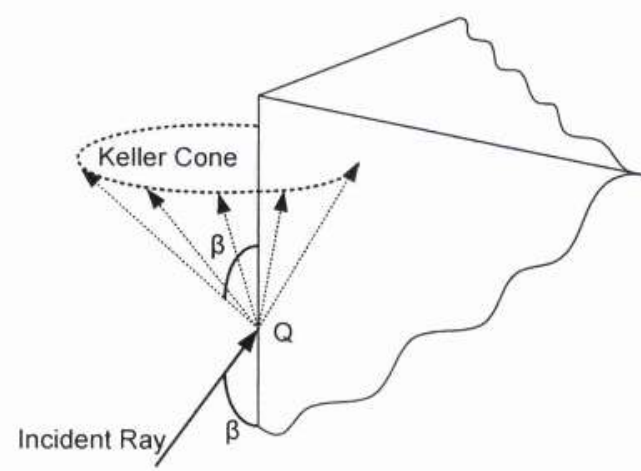

Figure 3-2: Generation of edge-diffracted rays

Now consider the situation in Figure 3-3. In Region 1 the field is the sum of direct and reflected rays, plus diffracted rays of negligible amplitude. In Region 2 there is no reflection, whereas in Region 3, which is the shadowed region, there is only a diffracted field. The diffraction coefficients calculated by GTD are discontinuous around the transition boundaries leading to incorrect field predictions close to those boundaries. In order to solve the problem of these discontinuities and predict the field correctly close to the transition boundaries, the original formulation of GTD was extended to the Uniform Theory of Diffraction (UTD). The extended theory yields continuous coefficients and is valid for all points in space.

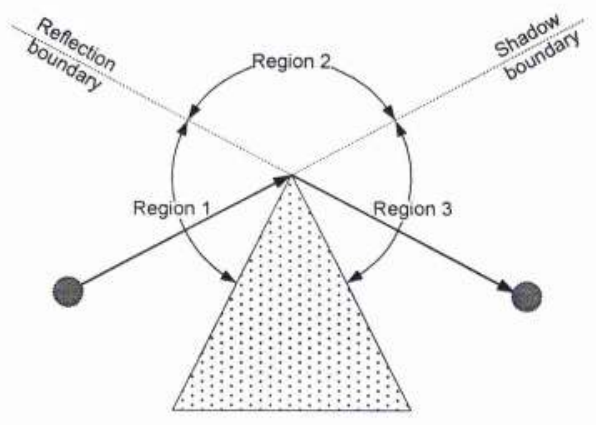

Figure 3-3: Regions around a wedge 
In 1974 Kouyoumjian and Pathak [26] used an asymptotic analysis (asymptotic solution of the Maxwell Equations) and found that by multiplying the diffraction coefficients by a transition function the diffracted fields remain bounded across the shadow boundaries. This transition function approaches zero as the diffraction coefficients approach singularity at the shadow boundaries eliminating the singularities. Their solution reduces to known asymptotic solutions for the wedge, and it was found to yield the first two or three terms in the asymptotic expansion of the diffracted fields.

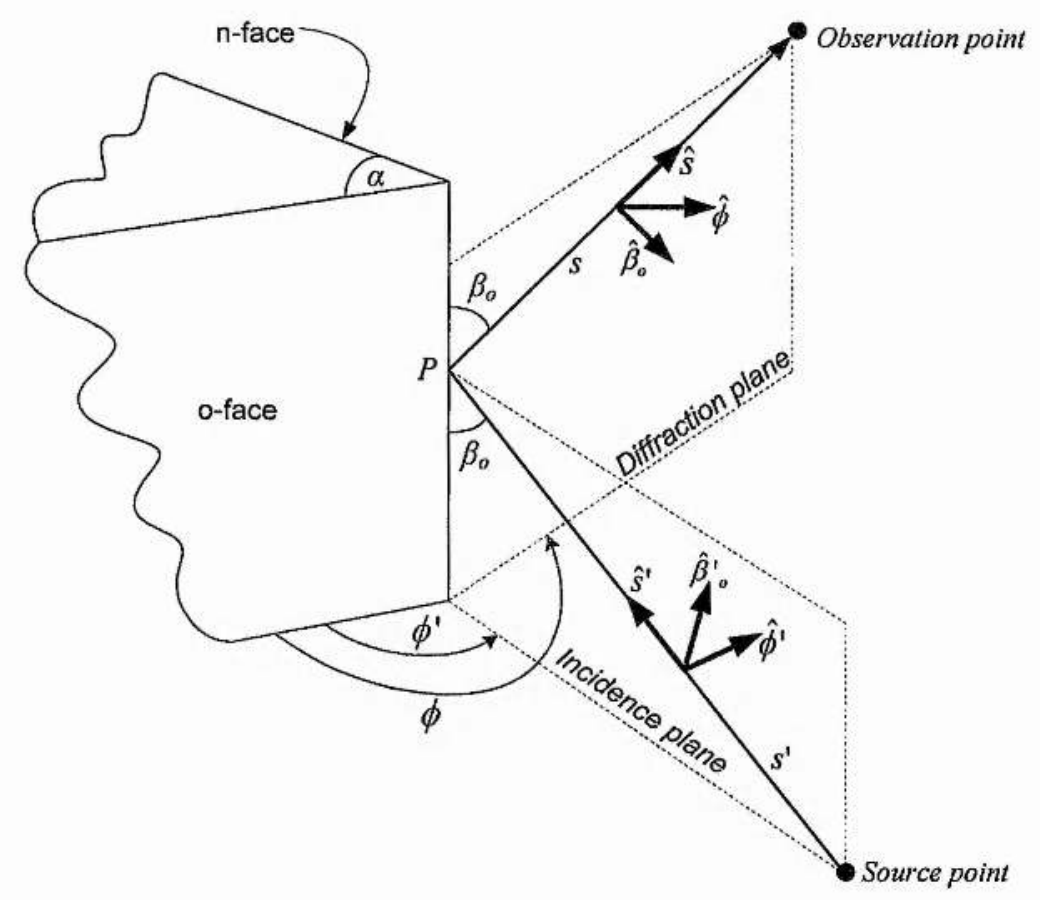

Figure 3-4: Three dimensional wedge diffraction Geometry

Figure 3-4 illustrates the three dimensional geometry and parameters used for calculating UTD coefficients. For any random observation point the diffracted field is given by,

$$
E_{d}=D \cdot A \cdot E_{P} e^{-j k s}
$$

where $E_{P}$ is the electric field at point $\mathrm{P}$ prior the diffraction, $k$ is the wavenumber, $s$ is the distance between the point $\mathrm{P}$ and the observation point, $A$ is the spreading factor describing the field variation along the diffracted ray given by:

$$
A=\left\{\begin{array}{l}
\frac{1}{\sqrt{s}} \text { for plane and cylindrical waves } \\
\sqrt{\frac{s^{\prime}}{s^{\prime}\left(s^{\prime}+s\right)}} \text { for spherical waves }
\end{array}\right.
$$


$D$ is the dyadic diffraction coefficient obtained by [10],

$$
D=-\hat{\beta}^{\prime}{ }_{o} \hat{\beta}_{o} D_{s}-\hat{\phi}_{o}^{\prime} \hat{\phi}_{o} D_{h}
$$

where the vectors are components of the edge coordinate system which is introduced for simplicity. These are defined as,

$$
\begin{array}{cc}
\hat{\phi}^{\prime}=\frac{-\hat{e} \times \hat{s}^{\prime}}{\left|\hat{e} \times \hat{s}^{\prime}\right|} \quad \hat{\beta}_{o}^{\prime}=\hat{\phi}^{\prime} \times \hat{s}^{\prime} \\
\hat{\phi}=\frac{-\hat{e} \times \hat{s}}{|\hat{e} \times \hat{s}|} \quad \hat{\beta}_{o}=\hat{\phi} \times \hat{s}
\end{array}
$$

$\hat{s}^{\prime}$ and $\hat{s}$ are unit vectors in the direction of the incident and diffracted ray respectively.

$D_{s}$ and $D_{h}$ are the soft and hard diffraction coefficients defined as,

$$
\begin{gathered}
D_{s, h}=D_{1}+D_{2}+R_{s, h}^{n} D_{3}+R_{s, h}^{o} D_{4} \\
D_{1}=\frac{-e^{-j \pi / 4}}{2 n \sqrt{2 \pi k} \sin \beta_{o}} \cot \left[\frac{\pi+\left(\phi-\phi^{\prime}\right)}{2 n}\right] F\left[k L a^{+}\left(\phi-\phi^{\prime}\right)\right] \\
D_{2}=\frac{-e^{-j \pi / 4}}{2 n \sqrt{2 \pi k} \sin \beta_{o}} \cot \left[\frac{\pi-\left(\phi-\phi^{\prime}\right)}{2 n}\right] F\left[k L a^{-}\left(\phi-\phi^{\prime}\right)\right] \\
D_{3}=\frac{-e^{-j \pi / 4}}{2 n \sqrt{2 \pi k} \sin \beta_{o}} \cot \left[\frac{\pi+\left(\phi+\phi^{\prime}\right)}{2 n}\right] F\left[k L a^{+}\left(\phi+\phi^{\prime}\right)\right] \\
D_{4}=\frac{-e^{-j \pi / 4}}{2 n \sqrt{2 \pi k} \sin \beta_{o}} \cot \left[\frac{\pi-\left(\phi+\phi^{\prime}\right)}{2 n}\right] F\left[k L a^{-}\left(\phi+\phi^{\prime}\right)\right]
\end{gathered}
$$

where $n=(2 \pi-a) / a$ is the wedge factor with $a$ being the wedge interior angle

$\beta_{o}$ is the incident (or the diffracted ray) and the edge and

$F(x)$ is the transition integral given by:

$$
\begin{gathered}
F(x)=2 j \sqrt{x} e^{j x} \int_{\sqrt{x}}^{\infty} e^{-j \tau^{2} d \tau} \\
a^{ \pm}\left(\phi, \phi^{\prime}\right)=2 \cos ^{2}\left[\frac{2 \pi n N^{ \pm}-\left(\phi \pm \phi^{\prime}\right)}{2}\right]
\end{gathered}
$$

$N^{ \pm}$is the integer that most nearly satisfies,

$$
2 \pi n N^{+}-\left(\phi \pm \phi^{\prime}\right)=\pi \text { or } 2 \pi n N^{-}-\left(\phi \pm \phi^{\prime}\right)=-\pi
$$




$$
L= \begin{cases}\frac{s^{\prime} s}{s^{\prime}+s} \sin ^{2} \beta_{o} & \text { for spherical wave incidence } \\ \frac{r^{\prime} r}{r^{\prime}+r} & \text { for cylindrical wave incidence } \\ s \sin ^{2} \beta_{o} & \text { for plane wave incidence }\end{cases}
$$

where the cylindrical wave of radius $r^{\prime}$ is normaly incident on the edge, and $r$ is the perpendicular distance of the field point from the edge.

$R_{s, h}^{o}$ and $R_{s, h}^{n}$ are the reflection coefficients for perpendicular and parallel polarisation for the oface with an incident angle $\phi^{\prime}$ and for the $\mathrm{n}$-face for an incident angle $n \pi-\phi$ respectively. They can be calculated using the boundary Fresnel coefficients in equations (2.26) and (2.28)

When diffraction coefficients are calculated they can be included in the calculation of the total electric field. Hence equation (3.5) becomes,

$$
E=\sum_{i} E_{o} A_{i}\left[\prod_{j=1}^{N_{R i}} R_{i, j} \prod_{m=1}^{N_{T i}} T_{i, m} \prod_{n=1}^{N_{D i}} D_{i, n}\right] \mathrm{e}^{-j \mathrm{kr}_{\mathrm{i}}}
$$

where, $N_{D i}$ diffractions of each ray path i respectively and $D_{i_{i} n}$ are the corresponding diffraction coefficients. $A_{i}$ is the spreading factor that accounts for the wavefront curvature as well as the curvature of the reflection and transmission boundaries. This formulation applies for paths that only one diffraction is encountered. For multiple-diffraction, the spreading factor of the individual diffracted components will change and requires special treatment [27].

\subsubsection{UTD Limitations}

Although the UTD provides a more accurate solution compared to the GTD by eliminating the singularities of the latter across the Incident Shadow boundary (ISB) and the Reflection Shadow Boundary (RSB), it still suffers from inaccuracies due to the fact that his asymptotic solution was derived for perfectly conducting infinite wedges. Wedges are usually formed by lossy dielectric interfaces which cannot be considered as infinite. Heuristic solutions have been derived by several authors [6][28][29] to handle lossy and finite-size wedges.

Also, UTD becomes inaccurate for lossy wedges in the deep shadow regions due to the fact that a transmitted ray exists across the wedge from another shadow boundary called the Transmission Shadow Boundary. Therefore, UTD can be considered as incomplete since it rectifies field singularities across the ISB and RSB but not TSB which is significant, since transmission is significant for hollow wedges as shown in Figure 3-5. The authors of [30] have developed and proposed an improved formulation of the diffraction coefficient in which appear two additional terms weighted by the transmission coefficients of each dielectric interface. 


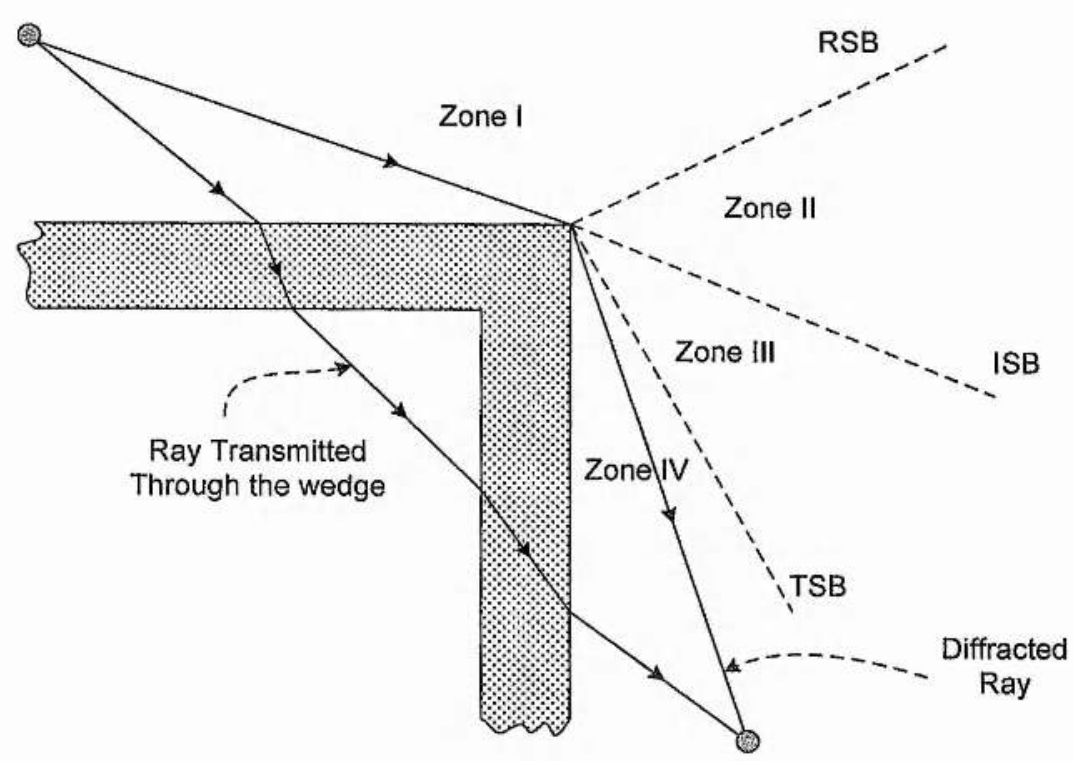

Figure 3-5: Hollow Wedge diffraction

\subsection{Environment Description}

The first step in a Ray Tracing Technique is to geometrically and morphologically represent the particular environment. Every deterministic propagation model requires geometric and morphological description of the environment. The more details that are included in the environment description the more accurate the model's predictions are.

\subsubsection{Geometric Description}

The geometric description of the environment is a description of geometry of the scenario under investigation in terms of the location and size of the obstructions (walls, windows, doors etc). It should be noted here that the more complex the geometric description is, the more computational overhead and more time will be required. Therefore, it is usually the case that depth of detail is traded for computation and time which means that only the most important features of the environment are described such as building and terrain data, where as small and mobile objects tend to be ignored. In the developed Ray Tracing model, objects which are large compared to the wavelength (like doors, windows etc) are taken into account (see Figure 6-1). The most common form of representing the objects in the environment is by using plane facets (polygonal plane facets). 


\subsubsection{Morphological Description}

The morphological description aims to describe the materials used in the particular scenario. For each geometrically described facet, the building material properties are defined since they significantly affect the reflection and transmission coefficients. These properties can be obtained by measurements or by simply providing the constitutive parameters of each material; these are:

- Relative permittivity $\varepsilon_{\mathrm{r}}$

" Relative permeability $\mu_{\mathrm{r}}$

- Conductivity $\sigma$

The above parameters are used to characterise smooth surfaces. In cases where the surface is characterised as rough, according to the Rayleigh criterion in (equation 2-58), the surface roughness parameter $\Delta \mathrm{h}$ should be specified as well [11].

\subsubsection{Faceted model}

Both the geometric and morphological descriptions will form the database for the model which can be stored in a separate file and loaded upon execution. This data might be either in raster or vector form. In the raster form the environment is subdivided into cells and each cell includes the corresponding information. In vector form the environment information is associated with geometric entities (lines or polygons). In case of deterministic modelling this information must be in a suitable vector form and if not, it should be transformed into one. All the data should be stored into a database structure using a facet model [11]. This means that every wall is represented by a polygon-shape (usually four-sided) facet which includes its geometric description (Cartesian coordinates of its vertices), its morphological description (constitutive parameters) and any other parameters that might be useful during the execution of the algorithm (e.g. if the facet contains other 'children' facets such as doors or windows which are defined as patches).

\subsection{Ray Tracing Algorithms}

The ray-tracing algorithms that have been proposed for high frequency propagation prediction mainly fall into two categories [11]. These are:

- Direct algorithms known as Ray Launching (or Shooting and Bouncing Rays Method SBR) and

- Inverse algorithms known as Image methods 


\subsubsection{Ray Launching Algorithm}

Ray launching, which can also be referred as Shooting and Bouncing Rays Method (SBR) is considered to be a direct (or forward) algorithm since it is considered to follow the ray's actual path from the transmitter to the receiver location. Actually, for a given transmitter location a large number of rays is transmitted in all directions which only exist if they satisfy a number of criteria.

These criteria are:

- The ray exists only if its field strength has not fallen below the receiver's sensitivity

- The ray exists only if it has encountered a number of interactions (reflections, refractions, diffractions) that is less than a user predefined threshold.

- A ray exists only if it can be received by a certain receiver location. In other words if the ray escapes the scene then it is discarded.

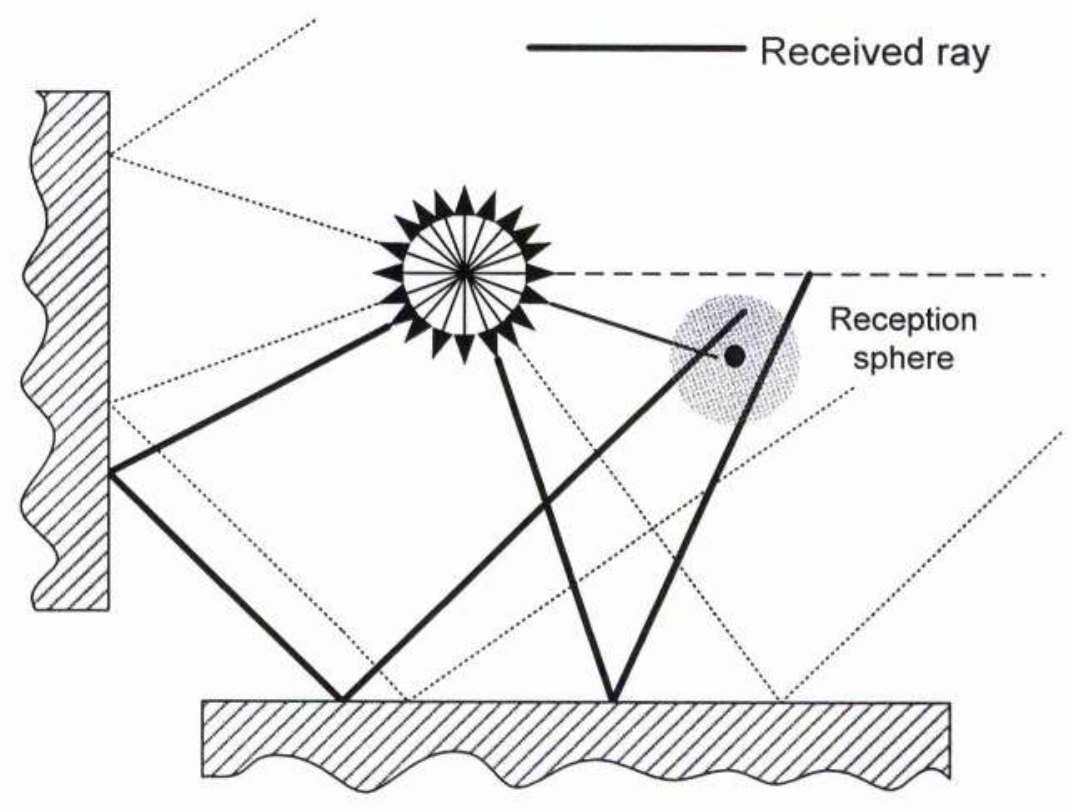

Figure 3-6: Ray Launching Concept

Every ray is a ray cone which occupies the same solid angle and as the tube advances its cross section increases. In this way the ray cones overlap and cover the whole spherical wavefront at the receiving location [32].

If we consider the receiver as a single point then the probability of rays leaving the transmitter to reach that point would be considerably small. For this reason the receiver is considered as a sphere (reception sphere), as shown in Figure 3-7, so as to increase the probability for capturing more rays. When a ray intersects the sphere, it is considered to be received and it 
contributes to the overall field strength of that point. The radius of the sphere is given by [11] [31]:

$$
R=\frac{\theta d}{\sqrt{3}}
$$

The ability to vary the radius of the reception sphere according to the receiver distance from the transmitter (d), accounts for the divergence of the rays from the transmitter and ensures the uniqueness of the captured rays. From simple geometry, one can observe that at distance $\mathrm{d}$ from the transmitter, adjacent rays are separated by distance $2 \theta d / \sqrt{3}$. Therefore the radius of the reception sphere is set to be one-half of that distance [31].

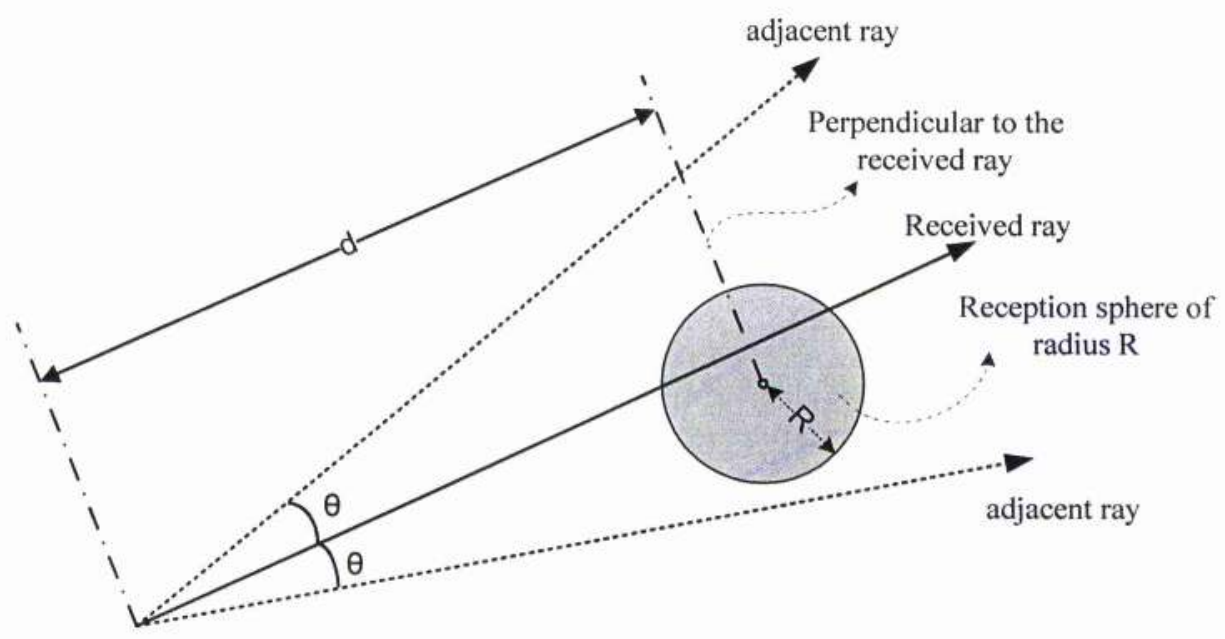

Figure 3-7: Reception Sphere

As the rays propagate they encounter a number of obstructions. When an object is hit, reflection, refraction, diffraction or scattering will occur depending on the geometry and morphology of the obstacle and the ray will follow different directions with different amplitude, phase and polarisation as described in section 2.4. This process continues for every ray as long as the aforementioned criteria are satisfied.

In indoor environments the number of ray traces increases significantly taking up CPU power and time. A convenient way is to arrange and store the whole propagation process in a "ray-tracing tree" [11]. In this tree, the branches correspond to emitted (launched) rays and the tree knots represent interactions with the environment obstacles (facets). An example is shown in Figure 3-8. 

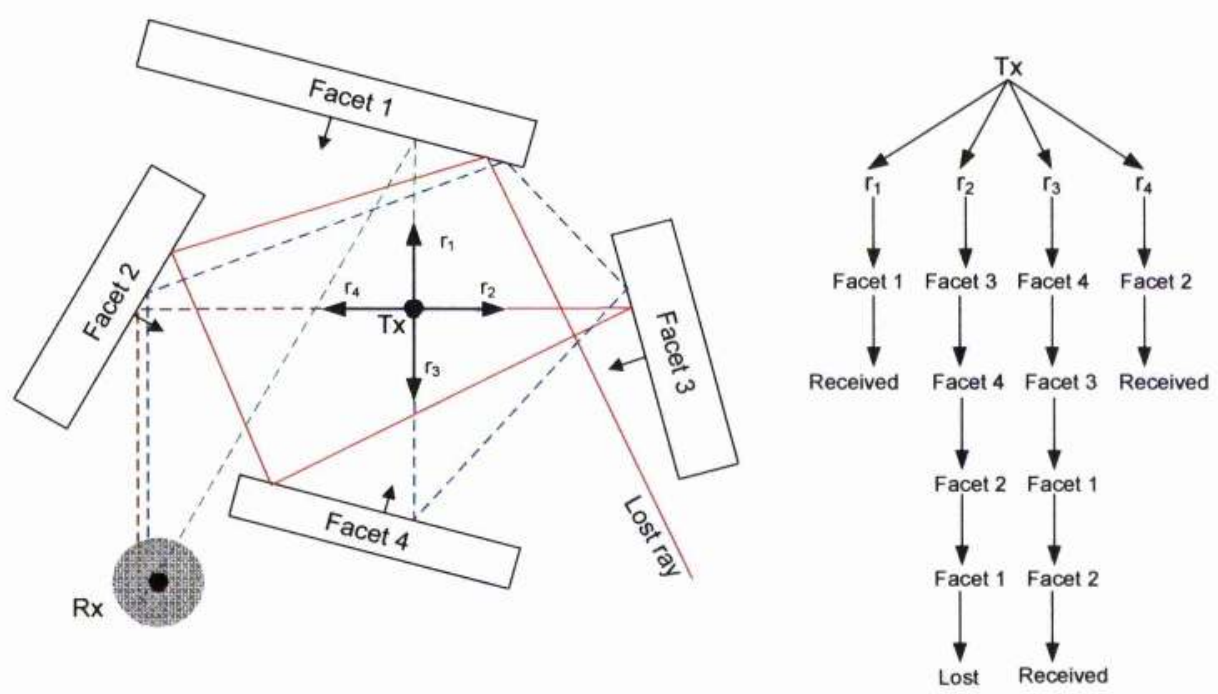

Figure 3-8: Ray-tracing tree example

The example above illustrates how the ray-tracing tree is constructed by considering reflections only. However in a practical situation, at every node of each ray branch the ray is decomposed into two children rays; the reflected and transmitted one; in order to consider refraction as well. Finally the total field strength at every receiver location can be obtained by summing up all the individual contributions.

\subsubsection{Image Method}

Compared to Ray-Launching, which is a direct method, the Image Method is referred as an inverse method. Instead of following the ray propagation path between the transmitter and the field points, this method tries to solve an inverse propagation problem. It is based on the image theory, where, for a given source point $(T x)$ and a facet, the reflected rays in the facet can be considered as being directly radiated from a virtual source called the image source (Im) which is symmetrical to the source with respect to the facet. This image theory is illustrated in Figure 3-9.

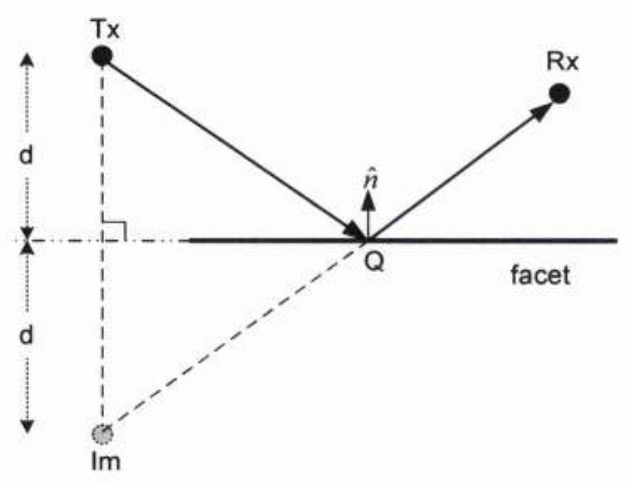

Figure 3-9:Image method for ray reflections 
Referring to the figure above, for a given receiver location $\mathrm{Rx}$, the point of interaction with the facet $\mathrm{Q}$ can be easily calculated as the intersection of the line Im-Rx and the facet. The facet can be either described in $2 \mathrm{D}$ as a line or in $3 \mathrm{D}$ as a polygon.

If we consider a scenario where only first order reflections are considered in an environment of $N$ facets then the maximum number of rays that can reach the receiver location is equal to $N$. However this is almost never the case in practical scenarios because of the following reasons [11]:

- Only observers that are located inside the reflection region of a given facet can receive reflections from it, as shown in Figure 3-10. In other words, when the point of intersection between the image source and the receiver location lies inside the facet.

- The reflected ray or the incident ray may be hidden by another facet.

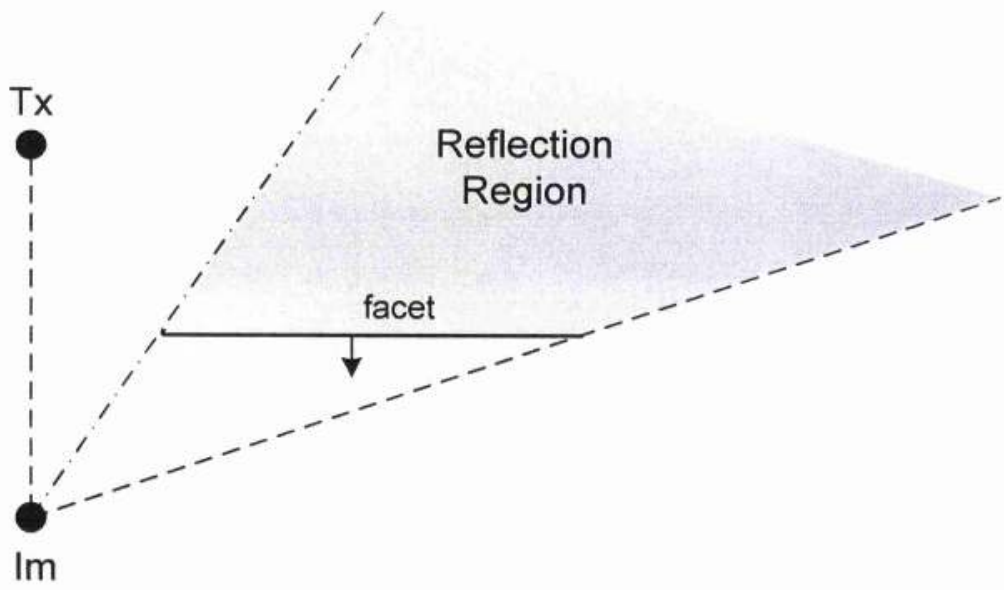

Figure 3-10: Reflection Region of a facet

Considering the same example as above, the $N$ first order images are considered as the transmitting sources for double-reflected rays. The images of the first order images are called second order images. The number of these images is $N(N-1)$ since the facets from which the parent first order images were derived are not considered. The case of double reflection is illustrated in Figure 3-11. 


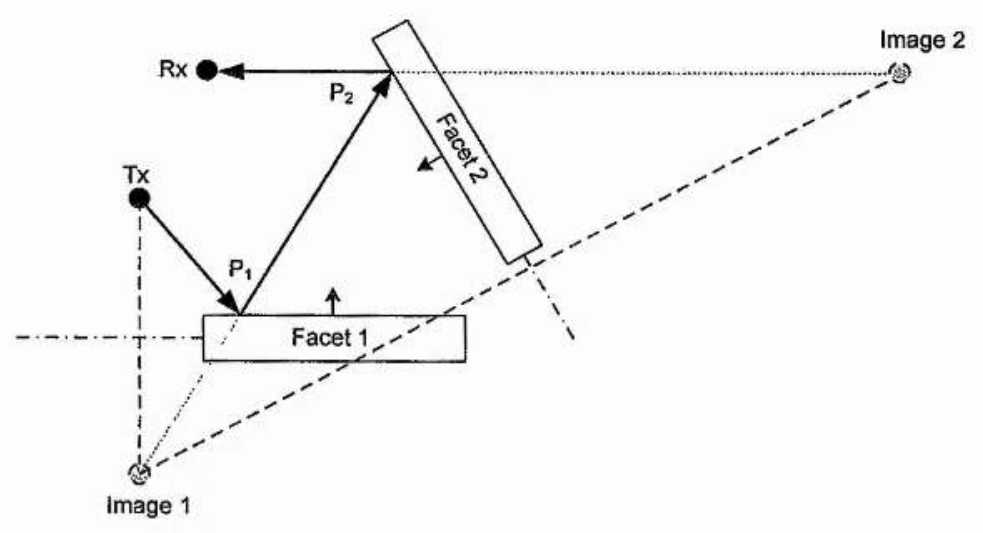

Figure 3-11: Image method for double reflections

For the above example the image of $T x$ due to Facet 1 is first determined (Image 1 ). Then the image of Image 1 due to Facet 2 is determined (Image 2). By connecting the $R x$ and Image 2 we can find the reflection point $P_{2}$. Now by connecting $P_{2}$ and $T x, P_{1}$ is determined. This process demonstrates why this method is referred as "backward" method since in order to find the reflection points one starts from the receiver and by using the image sources goes back to the transmitter. For double reflections to exist the following three conditions should be satisfied [11]:

1. The observation points should lie inside the reflection region of the second facet.

2. The second reflection point $P_{2}$ should be inside the reflection region of the first facet

3. None of the three paths $\left(T x-P_{1}, P_{1}-P_{2}, P_{2}-R x\right)$ should be hidden by any other facets.

The same procedure followed here for double reflections, is followed for multiple reflections. For $K$ reflections then the number of $K^{\text {th }}$-order images will be $N(N-1)^{K-1}$.

These images are arranged in a tree graph called the images tree, in a similar way as the ray-tracing tree in the ray-launching method. The first branching contains the $N$ first order images and for each branch $\mathrm{N}-1$ second order images exist. The $(\mathrm{N}-1)$-branching continues to account for multiple reflections.

However, not all of these images exist in practice. Therefore in order to reduce computation and save time, these rays should be discarded. Images are discarded if they satisfy the following criteria:

1. When a facet (Facet 2 ) is completely hidden by another facet (Face 1), as shown in Figure 3-12a, then its image can be discarded and therefore is not considered for further reflections (all its 'children' images are discarded as well).

2. If a facet (Facet 2) is completely outside the reflection region of another facet (Facet 1), as shown in Figure 3-12b, then its image can be discarded as well. By 
inspection we can see that this criterion fulfils the first one therefore for the purpose of simplicity the developed algorithm will use only this criterion.

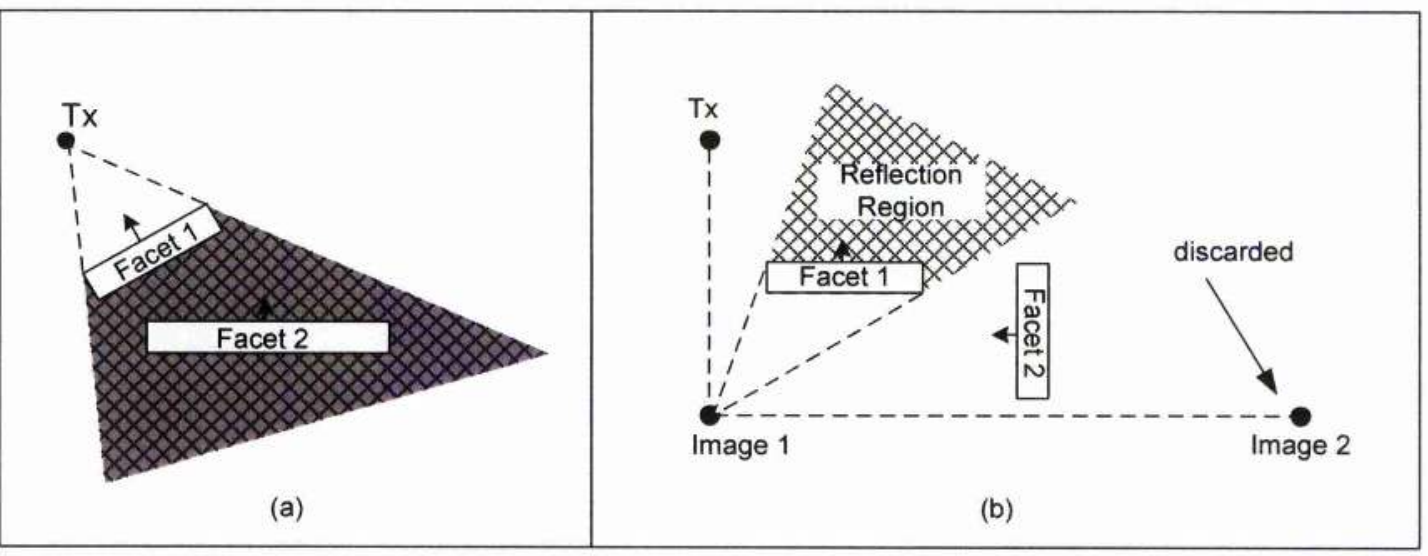

Figure 3-12: (a) Image discarded by hiding (b) Facet 2 out of reflection space of Facet 1

Using the above criteria the image tree can therefore be simplified by removing the images that do not exist in practice. The following figure illustrates an example of how the image tree is generated:

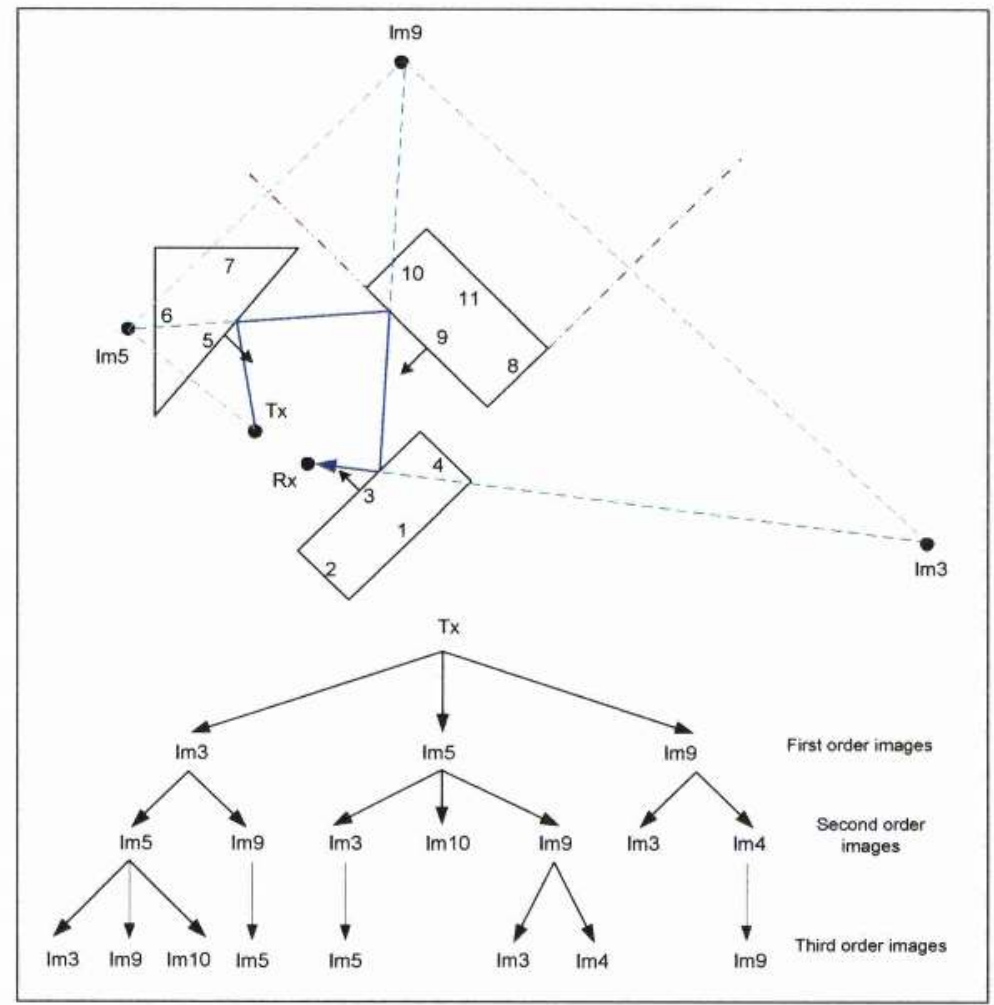

Figure 3-13: Image tree generation example 
Considering the example in Figure 3-13, for a given receiver position the tree is searched in a reverse manner. Referring to the receiver position shown in the example:

- The algorithm starts by considering one bottom level image ( $\operatorname{Im} 3)$ and determines the reflection point on the respective facet (facet 3 ). If the reflection point lies outside the facet limits, no valid path with the last interaction been reflection from this facet exists; thus the path is rejected and the next $3^{\text {rd }}$ order image is examined.

- If the reflection point lies inside the facet limits, a shadowing test is performed. If the path segment is shadowed by any other facet, the path is rejected and the next $3^{\text {rd }}$ order image is examined.

- If the path segment is not shadowed, the algorithm follows the branch to the previous level image. That is the case for the example, so the $2^{\text {nd }}$ order image Im9 is now considered.

- For the new image the same tests are performed. If any of these fail, the algorithm moves again to the next bottom level image. Otherwise the algorithm moves another level up and so on, until it reaches the transmitter. In that case the corresponding ray path is valid.

The image method is accurate for indoor propagation prediction but it becomes computationally inefficient if the number of facets included in the environment description is high, and the number of reflections considered is high as well [33]. This increases the amount of computation required and consequently the time to produce results. For these reason special acceleration techniques are most commonly used to increase the efficiency of this method.

\subsubsection{Acceleration Techniques}

Ray-Tracing acceleration algorithms are used to improve the efficiency of simulations with respect to computational power and time required to produce results, when the environmental descriptions of the scenarios under test become very complex. There are several ways to achieve acceleration which are classified into four categories.

1. Reducing the cost of intersecting a ray with the primitives used in the model. For this reason the faceted model discussed earlier is used, since the polygonal shaped polygon is a very simple primitive and hence the intersection test cost is reduced.

2. Reducing the total number of ray-facet intersection tests. Since shadowing tests must be carried out several times in the propagation model the total number of the required intersection tests increases dramatically. For this reason the number of shadowing tests 
can be reduced by discarding facets (or images if the image method is used as discussed in section 3.4.2) and therefore the number of ray-facet intersection tests is reduced.

3. Reduce the number of rays which are intersected with the environment. This technique which is more suitable for the Ray-Launching method aims to reduce the number of rays which are intersected with the environment by setting up a threshold related to the contribution of the ray to the overall result. If the contribution is less than the threshold then the ray is discarded.

4. Replacing individual rays with more general entities. The basic idea of such a technique is to trace many rays simultaneously. The problem of this technique is that it is not compatible with Geometrical Theory of Diffraction (GTD).

Some of the most common acceleration techniques are:

- The Binary Space Partitioning (BSP) Algorithm

- The Space Volumetric Partitioning (SVP) Algorithm

- The Angular Z-Buffer (AZB) Algorithm

- The Illumination Zones algorithm

Detailed information regarding these algorithms can be found in [11] [32] and [34].

\subsection{Factors influencing Ray-Tracing accuracy}

\subsubsection{GO and UTD Approximation}

As already mentioned Ray-Tracing is based on GO optics which is subject to some assumptions (see section 3.1), which yield to approximations in the calculation of the electric field. GO provide fairly good results for electrically large objects. However for scatterers having dimensions approaching the wavelength of operation Ray tracing will not provide accurate field predictions. This is also the reason why Ray-Tracing is usually referred as a high frequency approximation method [35]. This approximation might cause Ray-Tracing to fail, most probably for indoor environments where the internal clutter might consist of objects with small dimensions. To overcome this problem, the authors of [36] have proposed a hybrid Ray-Tracing FDTD (Finite Difference Time Domain) method to provide accurate results for small objects. The problem is that FDTD is computationally inefficient for large computational domains. In [36], FDTD was applied for small portions of the entire domain and near-to-near or near-to-far field transformation techniques were used to combine it with Ray-Tracing. 
In addition to the GO approximation, the original GTD and UTD diffraction coefficients presented by the authors of [25] and [26] are established for perfectly conducting wedges. However extensions of the UTD for wedges of finite conductivity have been introduced for a limited range of applications [37][38].

\subsubsection{Algorithm Implementation and Propagation mechanism factors}

Some inaccuracy factors arise from the algorithm implementation. For example, image method imposes large computation overhead when the environment clutter becomes increasing dense, thus practically not allowing the consideration of large number of rays or propagation mechanisms (reflections, refractions, diffractions). In [39], the relationship between the received power and different ray combinations and interactions are presented. In addition, the RayLaunching method may suffer from multiple receptions of unnecessary rays if the reception sphere becomes larger than its recommended size.

\subsubsection{Environment Description factors}

One of the most common sources of errors or inaccuracies in Ray-Tracing is due to the luck of sufficient environment description (both geometric and morphological). It is usually the case that only the most important features of the environment are described, neglecting small obstructions such as furniture, computers and small metallic obstructions which might have significant effect on radio propagation in indoor environments. Also, for outdoor environments, features that are difficult to geometrically describe, like trees and other moving objects such as cars are not considered to avoid computational overhead. These, in addition to rough surfaces which are usually approximated as smooth, give rise to scattering which might have significant effect on radio propagation prediction accuracy.

Apart from the obstructions geometric description, the morphological descriptions of their materials plays an important role on the accuracy of ray-tracing predictions. Since detailed morphological description of the environment's facets is not usually available standard materials are often assumed which is more likely that they electrically differ from the ones actually used in the building [52].

Finally, another important factor that significantly affects radio propagation modelling accuracy is that the environment interfaces are made of non-homogeneous materials. It is usually the case that building interfaces present some kind of periodicity which gives rise to a frequency selectivity and scattering. This natural building frequency selective behaviour is going to be presented in the next chapter. 


\subsection{Summary}

Deterministic techniques, especially through Ray Tracing, are the dominant techniques in predicting radio propagation in wireless environments. This chapter has dealt in detail with Ray Tracing, explaining its origins and evolution as well as the fundamental algorithms used.

Ray tracing is based on GO which is used to identify all the possible Rays from the transmitter to the receivers by utilising the previously presented Snell's Laws. However GO failed to predict the diffracted field into the shadow region therefore the Geometrical Theory of Diffraction has been proposed as its immediate solution. GTD however, presented discontinuities on the shadow boundary and hence its extension; the Uniform theory of Diffraction (UTD) has been proposed. UTD utilises a transition function that eliminates this discontinuity and correctly predicts the diffracted field on the shadow boundary.

The ray-tracing algorithms that have been proposed for high frequency propagation prediction mainly fall into two categories. These are the direct method (shooting and bouncing rays SBR) and the inverse method (image method). Each method has its advantages and disadvantages over the other, but they both have one common disadvantage; they can be both computationally inefficient and time-consuming. Therefore, special acceleration techniques exists that aim to minimise this computation.

Various factors exists that might affect radio propagation prediction through ray tracing. These include the fact that Ray Tracing is based on approximate methods (Geometric optics) which are subject to various limitations and assumptions. Also factors that relate to the algorithm implementation and the number and type of propagation mechanisms considered by the simulation may give rise to inaccuracies. Finally, but not least the level of detail that the environment description defines will produce some errors, since it is common practice that only the most important parts of the environment are defined in order to achieve lower computation load and time. Also all the walls are assumed to be homogeneous which is not usually the case, since most interfaces in a building environment are made of non-homogeneous materials. More specifically most walls have a periodic internal structure which gives rise to frequency dependent characteristics and propagation of higher modes at non-specular directions. This natural periodic behaviour of building interfaces is presented in the next chapter. 


\section{Chapter 4}

\section{Natural Building Frequency Selectivity}

The radio propagation theory and formulation presented so far was mainly concerned with smooth homogeneous wall structures, which are modelled as half space materials or slab and layered models assuming that transmission and reflection by building walls have a substantial specular component. However, in some cases, building interfaces are not homogeneous but can present some periodic variations that give rise to reflections in non-specular directions. The web and void design of the individual blocks and their arrangement within a building wall/interface creates a periodic structure, which exhibits frequency dependent transmission and reflection characteristics [40]. In their work, the authors of [40] have derived reflection and transmission characteristics of a typical concrete block wall for TE polarization. They have found out that at $900 \mathrm{MHz}$ only specular component exist but for frequencies above $1.2 \mathrm{GHz}$ they have observed the generation of non-specular components due to the excitation of first and second harmonics.

In addition to brick walls, reinforced concrete walls have significant impact on radio wave propagation in wireless environments. Such walls are typically made of a metallic mesh embedded in a thick layer of concrete. This mesh consists of straight bars joined perpendicularly to a give a planar periodic array of square grid for structural reinforcement. The authors of [41] and [42] have used electromagnetic (EM) methods (FEM and FDTD respectively) to study the reflections and transmission characteristics from such structures. They have observed resonances to occur in the reflection and transmission characteristics which depend on the mesh geometry, the wall thickness and the electrical properties of the materials used.

For higher frequencies of operation the standard ray tracing presented so far would be insufficient to provide accurate results for building interfaces that exhibit periodic variations. Higher order harmonics should be incorporated into prediction algorithms to account for this periodic behaviour. As already mentioned, hard EM methods such as FEM [41] and FDTD [42] can be used for this purpose. However, such methods currently make the analysis complicated, time-consuming and hard to follow large problems or areas of study. Holloway in his paper [43], has suggested a method of homogenization of the periodic structure in order to determine the effective material properties of composite structures. He assumes that a periodic wall has an equivalent N-layer homogeneous medium which can be easily solved for reflection and transmission coefficients using the multilayer model presented in section 2.4.1.3. However this 
method is only valid where the wavelength is larger or similar to the periodicity of the structure. Savov [44] has proposed a 2D Modal Transmission Line (2D-MTL) method to analyse both reinforced concrete and concrete block walls. His work shows that MTL is a simple, accurate and computationally efficient method to study the transmission and reflection characteristics of a periodic wall structure. However, only the TE polarised incident plane wave has been investigated in 2D incidence. Yang [45] has extended Savov's model for the more general 3D case. The formulation presented in [46] has been utilised to develop an RCWA model in order to illustrate the natural frequency selectivity that typical buildings might exhibit. This chapter presents simulation results from typical concrete masonry blocks as well as typical reinforced concrete walls. The occurrence of resonances in the reflection and transmission characteristics and the generation of non-specular components are presented.

\subsection{Rigorous Coupled Wave Analysis (RCWA) for periodic structures}

\subsubsection{Model Description}

The Rigorous Coupled-Wave Analysis (RCWA) was first proposed by Moharam and Gaylord in [47] for the analysis of planar grating diffraction. It is based on a state-space representation of the Maxwell differential equations. For the analysis of periodic building structures they suggested the utilisation of this method to calculate reflection and transmission characteristics as well as the scattering behaviour of the various propagating modes. For simplicity, a three-layer periodic structure, representing periodic concrete block is shown in Figure 4-1

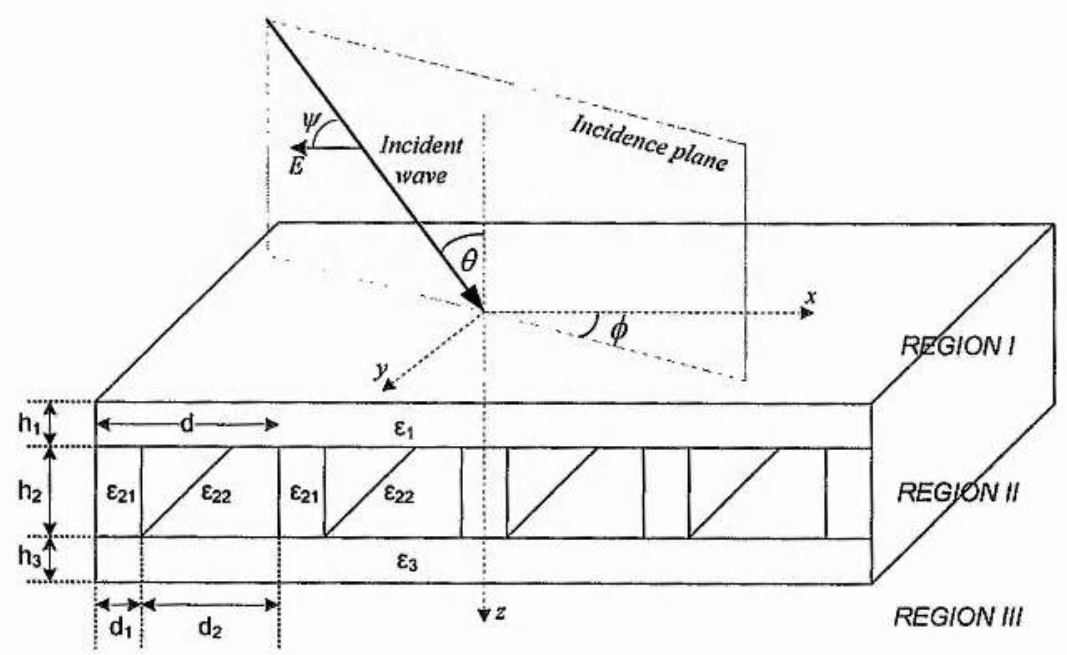

Figure 4-1: Geometry of 3D oblique incidence on a three-layer periodic structure 
The above figure represents a periodic slab, sandwiched between two homogeneous layers with dielectric constants $\varepsilon_{l}$ and $\varepsilon_{3}$ respectively. This layer, consists of two alternating homogeneous bars with dielectric constants $\varepsilon_{2 l}$ and $\varepsilon_{22}$, and widths $d_{1}$ and $d_{2}$ therefore exhibiting a permittivity $\varepsilon(x)=\varepsilon(x+d)$ along the $\mathrm{x}$-axis with periodicity $d=d_{1}+d_{2}$. For an $L$ number of layers $(L=3$ in this example), the overall structure thickness is $H_{L}=\sum h_{l}$ where $h_{l}$ is the thickness of the $l$ th layer. Region I and III (free space for this case) are the incident and exit regions where as Region II is the multilayer structure region. $\theta$ and $\phi$ are the elevation and azimuth angles of incidence for a linearly polarized time-harmonic plane wave obliquely incident on the structure. $\psi$ is the angle formed between the electric field vector and the incidence plane denoting the polarization of the incident wave ( $\psi=0^{\circ}$ for TM and $\psi=90^{\circ}$ for TE polarization).

Following the formulation presented in[47]-[49] an RCWA model has been developed to simulate structures which exhibit single periodicity (only along the $\mathrm{x}$-axis) such as concrete masonry block and reinforced concrete walls

\subsubsection{Regions I and III}

The incident electric field in Region I is given by:

$$
\vec{E}_{i n c}=\hat{u} e^{-j \vec{k}_{I}(x \hat{x}+y \hat{y}+z \hat{z})}
$$

, where $\hat{u}$ is the incident wave electric field vector given by:

$$
\hat{u}=(\cos \psi \cos \theta \cos \phi-\sin \psi \sin \phi) \hat{x}+(\cos \psi \cos \theta \sin \phi-\sin \psi \cos \phi) \hat{y}-(\cos \psi \sin \theta) \hat{z}
$$

$\vec{k}_{l}$ is the wave vector given by:

$$
\vec{k}_{I}=k_{o} n_{I}(\sin \theta \cos \phi \hat{x}+\sin \theta \sin \phi \hat{y}+\cos \theta \hat{z})
$$

$n_{I}$ is the Region I refractive index and $k_{o}=2 \pi / \lambda$ is the free space wave number. According to [49] the electric field in regions I and III are expressed as:

$$
\begin{aligned}
& \vec{E}_{l}=\vec{E}_{i n c}+\sum_{i} R_{i} e^{-j\left(k_{x i} x+k_{y} y-k_{I, z i} z\right)} \\
& \vec{E}_{I I}=\sum_{i} T_{i} e^{-j\left\{k_{x i} x+k_{y} y-k_{I I, z i}\left(z-H_{3}\right)\right\}}
\end{aligned}
$$

, where $R_{i}$ and $T_{i}$ are the electric field amplitudes of the $i$ th reflected wave in region I and the $i$ th transmitted wave in region III respectively, and,

$$
\begin{gathered}
k_{x i}=k_{o} n_{I} \sin \theta \cos \phi-\frac{i 2 \pi}{d} \text { for }(i=\cdots-2,-1,0,+1,+2 \cdots) \\
k_{y}=k_{o} n_{I} \sin \theta \sin \phi
\end{gathered}
$$




$$
k_{l, z i}=\left\{\begin{array}{l}
\sqrt{\left(k_{o} n_{l}\right)^{2}-k_{x i}^{2}-k_{y}^{2}} \text { for }\left(k_{x i}^{2}-k_{y}^{2}\right)<\left(k_{o} n_{l}\right)^{2} \\
-j \sqrt{k_{x i}^{2}+k_{y}^{2}-\left(k_{o} n_{l}\right)^{2}} \text { for }\left(k_{x i}^{2}-k_{y}^{2}\right)>\left(k_{o} n_{l}\right)^{2}
\end{array}\right.
$$

Equation 4.8 calculates $k_{l, z i}$ in regions $l=\mathrm{I}$ and $l=\mathrm{III}$ for a propagating mode (real positive) or an evanescent mode (negative imaginary).

The corresponding magnetic fields can be obtained using:

$$
\vec{H}=\left(\frac{j}{\omega \mu}\right) \nabla \times \vec{E}
$$

, where $\omega$ is the angular frequency and $\mu$ is the permeability of the region. In order to calculate the direction of the various reflected and transmitted propagating modes $(i)$ the following equations apply:

$$
\begin{gathered}
\varphi_{i}=\tan ^{-1}\left(\frac{k_{y}}{k_{x i}}\right) \\
\vartheta_{i}=\tan ^{-1}\left[\frac{\sqrt{k_{x i}^{2}+k_{y}^{2}}}{\operatorname{Re}\left(k_{l, z i}\right)}\right]
\end{gathered}
$$

, where $\varphi_{i}$ and $\vartheta_{i}$ represent the inclination of the $i$ th mode with respect to the $x$ axis and $z$ axis respectively.

\subsubsection{Region II}

In Region II, the electric and magnetic field in each $l$ th layer can be expressed as a series of space harmonics as:

$$
\begin{gathered}
\vec{E}_{l}=\sum_{i}\left(S_{l x i}(z) x+S_{l y i}(z) y+S_{l z i}(z) z\right) e^{-j\left(k_{x i} x+k_{y} y\right)} \\
\vec{H}_{l}=-j \sqrt{\frac{\varepsilon_{o}}{\mu_{o}}} \sum_{i}\left(U_{l x i}(z) x+U_{l y i}(z) y+U_{l z i}(z) z\right) e^{-j\left(k_{x i} x+k_{y} y\right)}
\end{gathered}
$$

, where $S_{l i}(z)$ and $U_{l i}(z)$ are the space harmonic field amplitudes which are used so that the Maxwell equations in the $l$ th layer are satisfied

$$
\nabla \times \vec{E}_{l}=-j \omega \mu_{o} \vec{H}_{l} \text { and } \nabla \times \vec{H}_{l}=j \omega \varepsilon_{o} \varepsilon_{l}(x) \vec{E}_{l}
$$

, where $\varepsilon_{l}(x)$ is the $l$ th layer relative permittivity. It is a periodic function expandable in a Fourier series for a periodic layer as:

$$
\varepsilon_{l}(x)=\sum_{n} \varepsilon_{l, n} e^{j 2 \pi n x / d} \quad \text { and } \quad \varepsilon_{l, n}=\frac{1}{d} \int_{-d / 2}^{d / 2} \varepsilon_{l}(x) e^{-j 2 \pi n x / d} d x
$$


Similarly the inverse permittivity is defined as:

$$
\varepsilon_{l}^{-1}(x)=\sum_{n} a_{l, n} e^{j 2 \pi n x / d} a_{l, n}=\frac{1}{d} \int_{-d / 2}^{d / 2} \varepsilon_{l}^{-1}(x) e^{-j 2 \pi n x / d} d x
$$

For the geometry shown in Figure 4-1 the coefficients of the Fourier series of the relative permittivity are obtained by:

$$
\varepsilon_{l, n}=\left\{\begin{array}{c}
\frac{\left(\varepsilon_{21} d_{1}+\varepsilon_{22} d_{2}\right)}{d} \text { for } n=0 \\
\frac{\left(\varepsilon_{21}-\varepsilon_{22}\right) \sin \left(\frac{\pi n d_{1}}{d}\right)}{\pi n} \text { for } n \neq 0
\end{array}\right.
$$

Substituting Eq. (4.12) and (4.13) into (4.14), and eliminating the z-axis components, results in an infinite set of $1^{\text {st }}$ order coupled wave equations, which can be written in matrix form by truncating infinite sums in order to numerically solve the following set of coupled differential equations:

$$
\frac{d}{d x}\left[\begin{array}{l}
S_{l, y} \\
S_{l, x} \\
U_{l, y} \\
U_{l, x}
\end{array}\right]=\left[\begin{array}{llrl}
0 & 0 & P_{l, 11} & P_{l, 12} \\
0 & 0 & P_{l, 21} & P_{l, 22} \\
Q_{l, 11} & Q_{l, 12} & 0 & 0 \\
Q_{l, 21} & Q_{l, 22} & 0 & 0
\end{array}\right]\left[\begin{array}{l}
S_{l, y} \\
S_{l, x} \\
U_{l, y} \\
U_{l, x}
\end{array}\right]
$$

,where for $I$ being an identity matrix,

$$
\begin{gathered}
P_{l}=\left[\begin{array}{ll}
P_{l, 11} & P_{l, 12} \\
P_{l, 21} & P_{l, 22}
\end{array}\right]=\left[\begin{array}{cc}
k_{y} \varepsilon_{l}^{-1} k_{x} & I-k_{y} \varepsilon_{l}^{-1} k_{y} \\
k_{x} \varepsilon_{l}{ }^{-1} k_{x}-I & -k_{x} \varepsilon_{l}^{-1} k_{y}
\end{array}\right] \\
Q_{l}=\left[\begin{array}{ll}
Q_{l, 11} & Q_{l, 12} \\
Q_{l, 21} & Q_{l, 22}
\end{array}\right]=\left[\begin{array}{cc}
k_{x} k_{y} & a_{l}^{-1}-k_{y}^{2} \\
k_{x}^{2}-\varepsilon_{l} & k_{x} k_{y}
\end{array}\right]
\end{gathered}
$$

$k_{x}$ and $k_{y}$ are diagonal matrices with elements $k_{y} / k_{o}$ and $k_{x i} / k_{o}$ respectively. $\varepsilon_{l}$ and $a_{l}$ are matrices formed by permittivity and inverse permittivity $\varepsilon_{l, i p}=\varepsilon_{l, i-p}$ and $a_{l, i p}=a_{l, i-p}$, (where $p=i-n$ ) respectively. $l, k_{x}, k_{y}, \varepsilon_{l}$ and $a_{l}$ are $N \times N$ matrices where $N$ is the number of the space harmonics retained in the field. Also, the $4 N \times 4 N$ matrix in equation (4.17) can be further reduced to two $N \times N$ matrices as:

$$
\begin{gathered}
\frac{d^{2} U_{l, x}}{d z^{\prime 2}}=\left[k_{y}^{2}+A\right]\left[U_{l, x}\right] \\
\frac{d^{2} S_{l, x}}{d z^{\prime 2}}=\left[k_{y}^{2}+B a_{l}^{-1}\right]\left[S_{l, x}\right]
\end{gathered}
$$

, where $A=k_{x}^{2}-\varepsilon_{l}$ and $B=k_{x} \varepsilon_{l}^{-1} k_{x}-I$. This reduces the computational time of the eigenvalue problem by a factor of 8 [49].Also, $z^{\prime}=k_{o} z$. When the layer $l$ is homogeneous (e.g. layer 1 and 3 in Figure 4-1), $\varepsilon_{l}$ and $a_{l}$ become diagonal matrices with elements $\varepsilon_{l}$ and $1 / \varepsilon_{l}$ respectively. 


\subsubsection{Truncation of Matrix Computation}

The matrices involved must be truncated to finite ones when carrying out the numerical computations. The truncated finite matrices should allow for accurate calculation of reflected and transmitted waves and to be tractable for numerical calculation.

Regarding the truncation process, it is generally accepted that more modes are needed for TM than TE polarisation [50]. A periodic layer with larger dimensions compared to the wavelength, and larger differences between the dielectric constants of the materials forming the periodic layer, needs a larger number of modes. Also, a metallic periodic layer needs a larger number of modes than a dielectric periodic layer. Therefore, a study of convergence is suggested to be performed for each particular case, to decide on the required number of modes to be retained in the computation.

\subsubsection{Boundary Conditions, Reflection and Transmission Coefficients}

In order to calculate the reflection and transmission coefficients in Regions I and III, the tangential electric and magnetic fields are matched to each boundary. The field equations at each boundary can be solved simultaneously for the TE and TM components of the reflected field in Region I $\left(R_{s i}, R_{p i}\right)$ and the transmitted field in Region III $\left(T_{s i}, T_{p i}\right)$. The standard transmittance matrix approach for propagating the field matching is known to produce numerical instability because of the presence of evanescent fields, even in the case of simple uniform multilayer systems. These evanescent fields which are associated with total internal reflection or losses, result in "ill-conditioned" transmittance matrices. The inversion of such an "ill-conditioned" matrix will result in a matrix with numerically large diagonal elements that cannot be represented with sufficient numerical accuracy because of the finite precision (truncation error) of digital computers. This numerical inaccuracy will produce numerical instability in the calculated transmitted and reflected fields. Moharam and Gaylord have proposed an enhanced transmittance approach [48] to overcome this instability. In this approach, the matrix to be inverted is written as a product of two matrices and with proper manipulation the numerical instability is eliminated. This approach [48] has been used in the developed RCWA model to provide efficient and stable numerical calculation for the reflection and transmission characteristics in periodic structures.

After computing $R_{s i}, R_{p i}$ and $T_{s i}, T_{p i}$ the reflection and transmission coefficients can be computed using:

$$
\begin{aligned}
& R_{i}=\left|R_{s i}\right|^{2} \operatorname{Re}\left(\frac{k_{I, z i}}{k_{I} \cos \theta}\right)+\left|R_{p i}\right|^{2} \operatorname{Re}\left(\frac{k_{l, z i}}{n_{I}^{2} k_{I} \cos \theta}\right) \\
& T_{i}=\left|T_{s i}\right|^{2} \operatorname{Re}\left(\frac{k_{I I l, z i}}{k_{I} \cos \theta}\right)+\left|T_{p i}\right|^{2} \operatorname{Re}\left(\frac{k_{I I l, z i}}{n_{I I}^{2} k_{I} \cos \theta}\right)
\end{aligned}
$$




\subsubsection{Model Verification}

In order to verify the validity of the developed RCWA model, results produced for a simple single periodic structure have been compared with results obtained from a commercial EM simulator; the Microwave Studio by CST. The parameters used in this case are: $h_{1}=h_{3}=$ $13.5 \mathrm{~mm}, h_{2}=126 \mathrm{~mm}, d_{1}=40 \mathrm{~mm}, d_{2}=110 \mathrm{~mm}, d=150 \mathrm{~mm}, \varepsilon_{1}=\varepsilon_{3}=\varepsilon_{21}=3+$ $j 0.03$ and $\varepsilon_{22}=1$. Reflection and Transmission coefficients for a frequency range between $0.5-2$ $\mathrm{GHz}$ have been simulated for two angles of incidence $\left(\theta=0^{\circ}, \phi=0^{\circ}\right.$ and $\left.\theta=45^{\circ}, \phi=0^{\circ}\right)$ for TE polarization $\left(\psi=90^{\circ}\right)$. Prior to that, a convergence study was carried out to decide the required number of modes to be used in the calculation of the reflection and transmission coefficients. Results in Figure 4-2 suggest that more than 15 modes need to be considered for transmission coefficient convergence. Figure 4-3 demonstrates a perfect agreement between the results obtained with the 1D-RCWA model and Microwave Studio. This comparison has provided the first indication of the frequency selectivity of periodic structures. The first step into this investigation was to study commonly used building periodic structures. One of them is the masonry block.

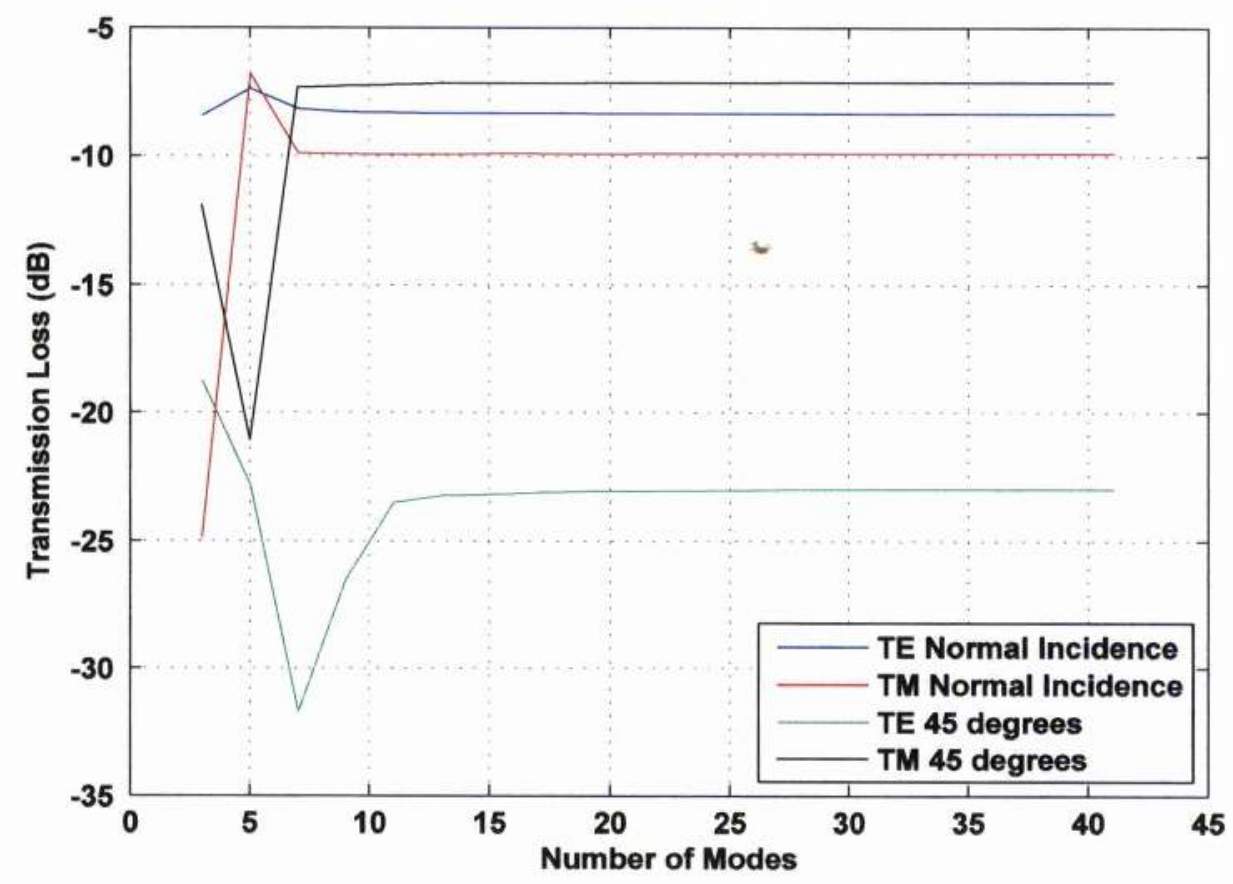

Figure 4-2: Convergence study of transmission loss versus the number of modes for the structure used for model verification at $2 \mathrm{GHz}$ 


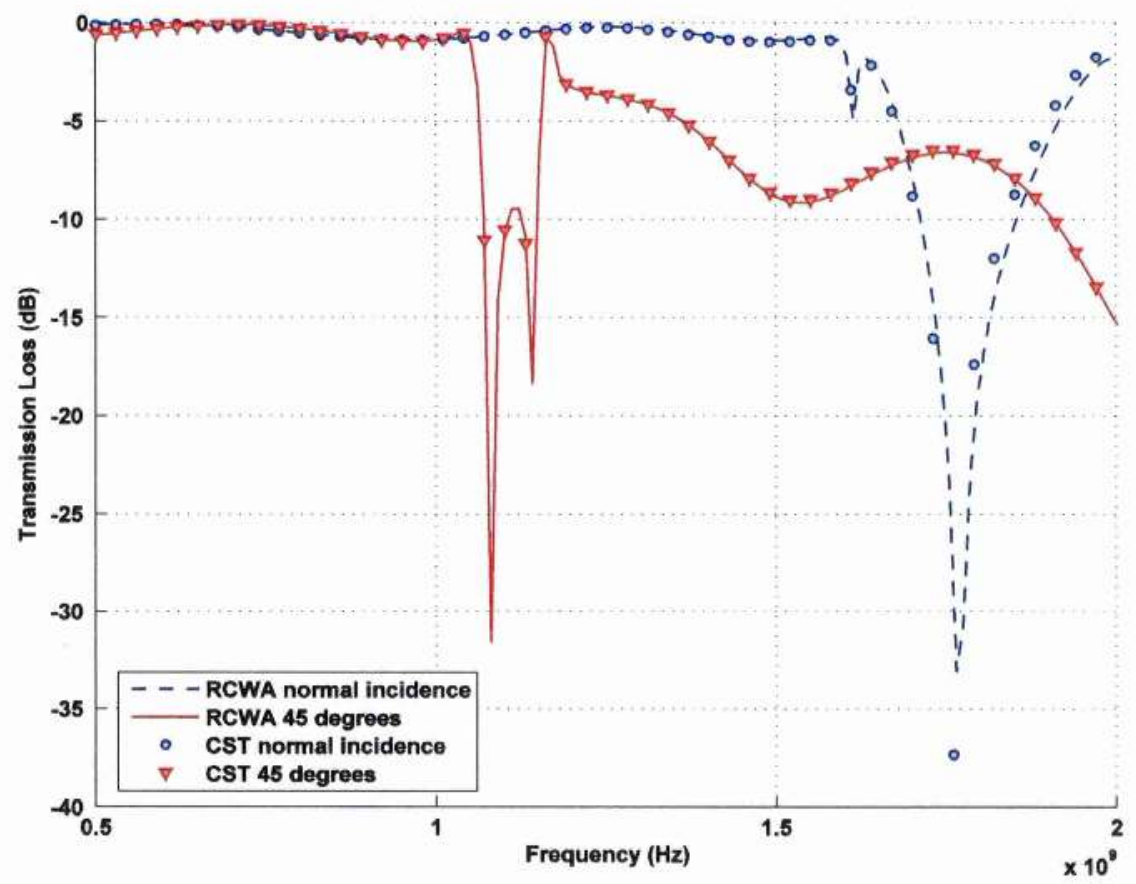

(a)

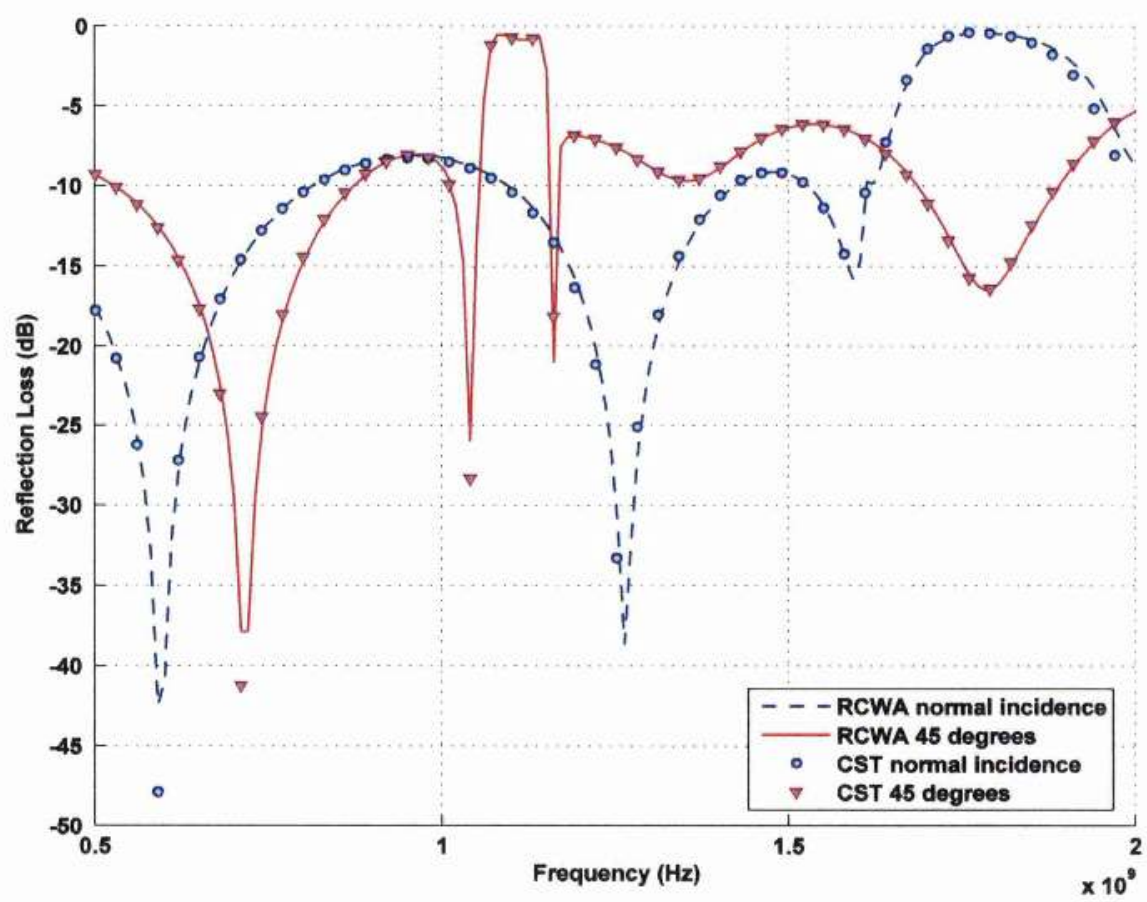

(b)

Figure 4-3: Transmission/Reflection Coefficients obtained with 1D-RCWA model and CST MWS $\left(\theta=0^{\circ}, \phi=0^{\circ}\right.$ and $\theta=45^{\circ}, \phi=0^{\circ}$, TE pol.) 


\subsection{Concrete Masonry Block Structures}

Masonry blocks include holes (air pockets), set towards either end of the block, a central thin rectangular slot and a cutout on one end usually used for window frame setting. A typical masonry block is shown in Figure 4-4. The thin rectangular slot and the cutout have been neglected for simplicity. Figure 4-5 demonstrates through CST simulations, the minor effect that this simplification has on the transmission loss through an 8-inch masonry block wall at normal incidence. This structure falls into the category of single periodicity structures since it is periodic only in the $x$-axis direction.

Standardisation includes five sizes of the above masonry block. These are shown in Table 4-1. According to BS 6073-2:1981 the cross sectional area of the cavity of a masonry block should not be less than $25 \%$ of the overall block cross sectional area. The values shown in Table 4-1 refer to masonry blocks with $40 \%$ cavity.

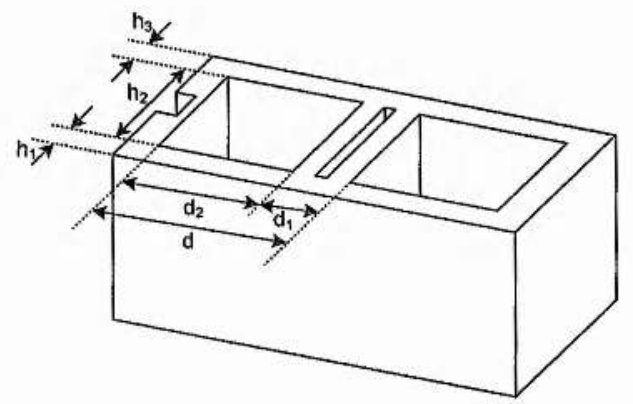

Figure 4-4: Masonry Block

\begin{tabular}{ccccccc}
\hline Type & $\mathbf{h 1}(\mathbf{m m})$ & $\mathbf{h 2}(\mathbf{m m})$ & $\mathbf{h 3}(\mathbf{m m})$ & $\mathbf{d}(\mathbf{m m})$ & $\mathbf{d 1}(\mathbf{m m})$ & $\mathbf{d 2}(\mathbf{m m})$ \\
\hline 4 inch & 21 & 43 & 21 & 198 & 43 & 155 \\
6 inch & 29 & 85 & 29 & 198 & 58 & 140 \\
8 inch & 34 & 126 & 34 & 198 & 70 & 128 \\
10 inch & 39 & 168 & 39 & 198 & 76 & 122 \\
12 inch & 41 & 213 & 41 & 198 & 82 & 116
\end{tabular}

Table 4-1: Typical sizes of concrete masonry blocks

The first question that someone would ask is: does this structure periodicity cause significant change on the propagation characteristics of an impinging propagating wave? This question is going to be answered through various simulations carried out for an 8-inch masonry block interface. The dielectric constant for concrete was set to $\varepsilon_{1}=\varepsilon_{2}=\varepsilon_{21}=7-0.3 j$ where as the cavity was left empty, hence $\varepsilon_{22}=1$. All the simulations have been carried out using a fixed 
complex dielectric permittivity, assuming that the material dispersion in the constitutive parameters for a frequency range of $0.5-3 \mathrm{GHz}$ has min effect on the results. 21 modes have been considered into the calculation of the reflection and transmission coefficients as suggested by the convergence study shown in Figure 4-6.

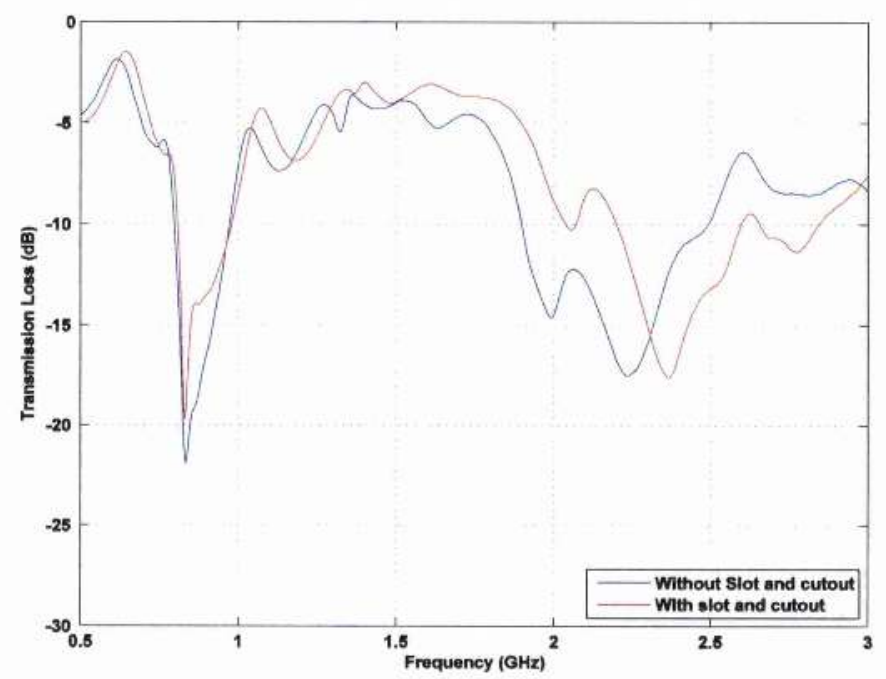

Figure 4-5: Effect of neglecting the slot and cutout for an 8-inch masonry block at normal incidence.

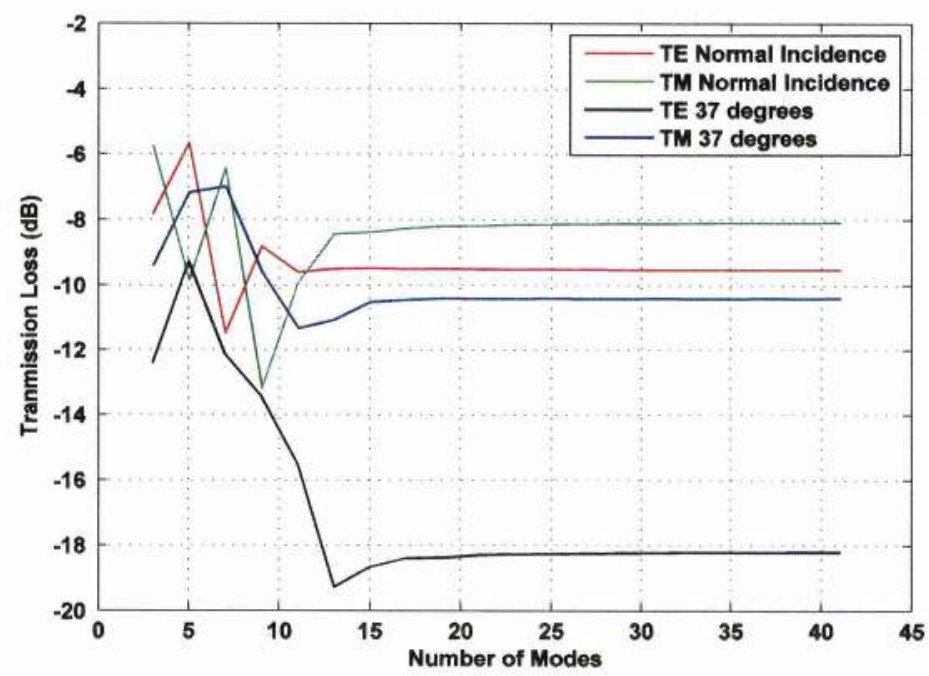

Figure 4-6: Convergence Study for an 8-inch masonry Concrete block at $3 \mathrm{GHz}$

Figure 4-7 illustrates a comparison between the reflection/transmission characteristics of a periodic concrete masonry block wall and a homogeneous concrete wall for TE polarization under an oblique angle of incidence $\left(\theta=37^{\circ}, \phi=0^{\circ}\right)$. It is obvious that strong resonances arise when the structure becomes periodic. For this particular example the effect is more significant for the transmission characteristics, where resonances up to $-40 \mathrm{~dB}$ occur. In some cases, masonry 
blocks are also filled with mortar for higher strength and insulation. The dielectric constant of mature mortar is $\varepsilon_{22}=5.2-j 1.2$ [51]. Figure 4-7 also shows the effect of filling the air pockets with mortar. It is observed that unlike the case where the pockets are left empty, the frequency response is not changed significantly compared to the homogeneous brick. This happens because the dielectric constant of mortar is similar to brick.

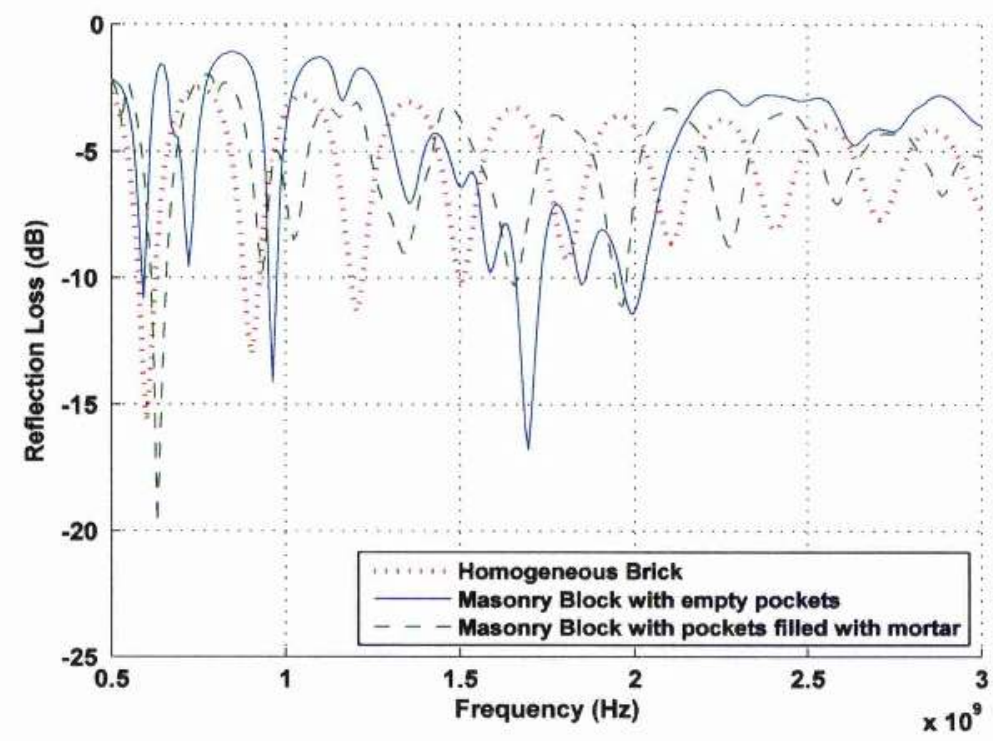

(a)

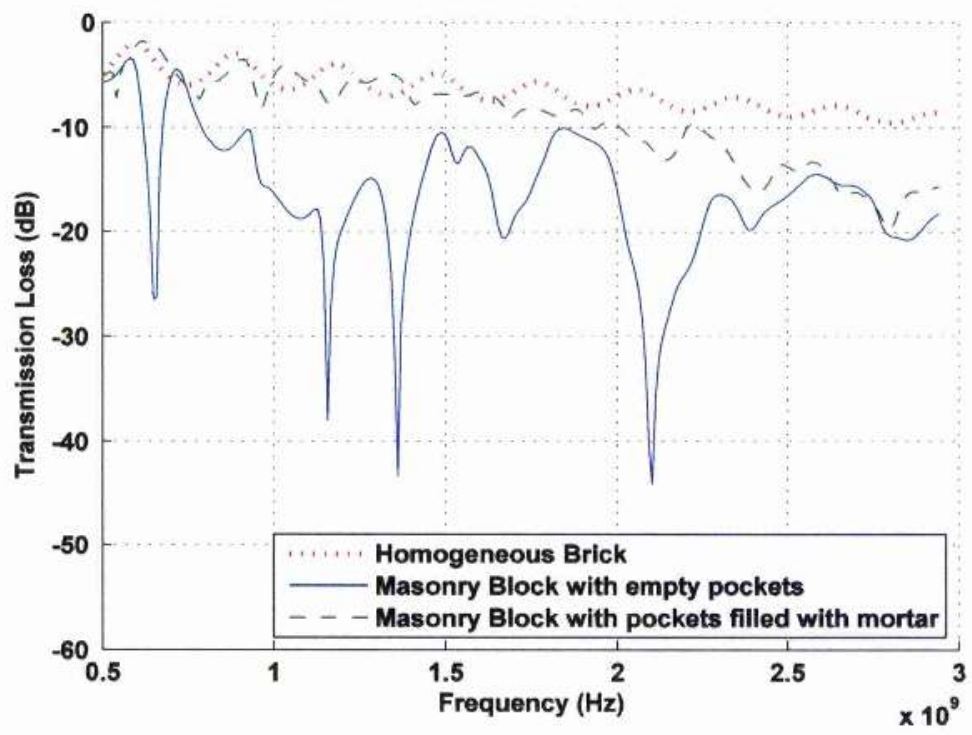

(b)

Figure 4-7: Transmission/Reflection Coefficients for an 8-inch masonry block $\left(\theta=37^{\circ}, \phi=0^{\circ}\right.$, TE pol. $)$ 
Following the findings of other researchers [41], [42], when the frequency increases, higher modes will begin to propagate in the air regions in either side of a periodic structure.

Figure 4-8 illustrates the transmission loss of various propagating modes as a function of frequency. For frequencies below $950 \mathrm{MHz}$ only the specular component propagates (mode 0 ). At $950 \mathrm{MHz}$ mode -1 is excited where as mode -2 and -3 start to propagate at $1.9 \mathrm{GHz}$ and $2.85 \mathrm{GHz}$ respectively. It is also observed that, for this angle of incidence, mode -1 carries more power than the specular component, for frequencies up to $2.46 \mathrm{GHz}$. Propagation of mode 0 is always along the specular direction $\left(37^{\circ}\right)$ where as higher order modes propagate in different directions. This is shown in Figure 4-9 which shows the transmitted angles of various modes for various frequencies.

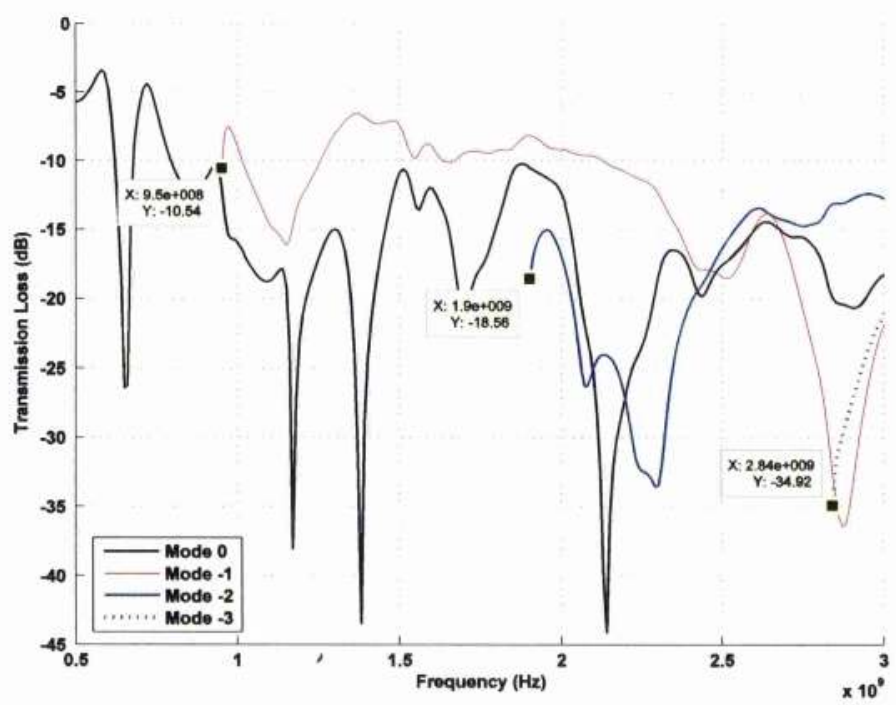

Figure 4-8: Transmission loss of various propagating modes, $\left(\theta=37^{\circ}, \phi=0^{\circ}, \mathrm{TE}\right.$ pol.) for a masonry block wall

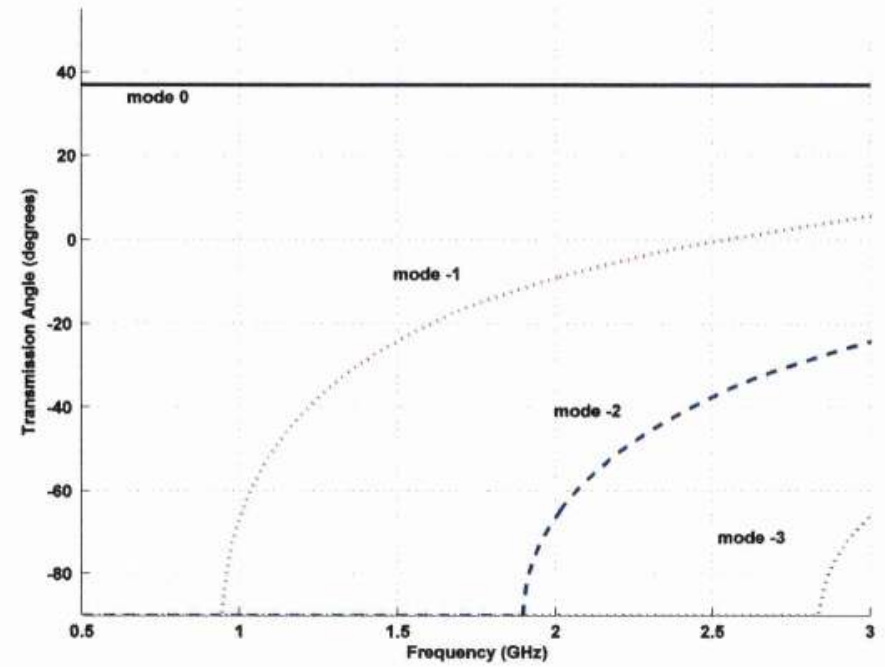

Figure 4-9: Transmission angles of various modes for various frequencies for masonry block wall 
Figure 4-10 shows the reflected and transmitted waves for each mode for $\left(\theta=37^{\circ}, \phi=\right.$ $0^{\circ}$ ) at $2 \mathrm{GHz}$. The thick black line is the incident wave. It is clear, that the higher order modes have significant impact on the radio propagation since they give rise to scattering. It is interesting that the dominant transmitted-through component is not along its specular direction (i.e mode 0 ) but along the direction of mode -1 .

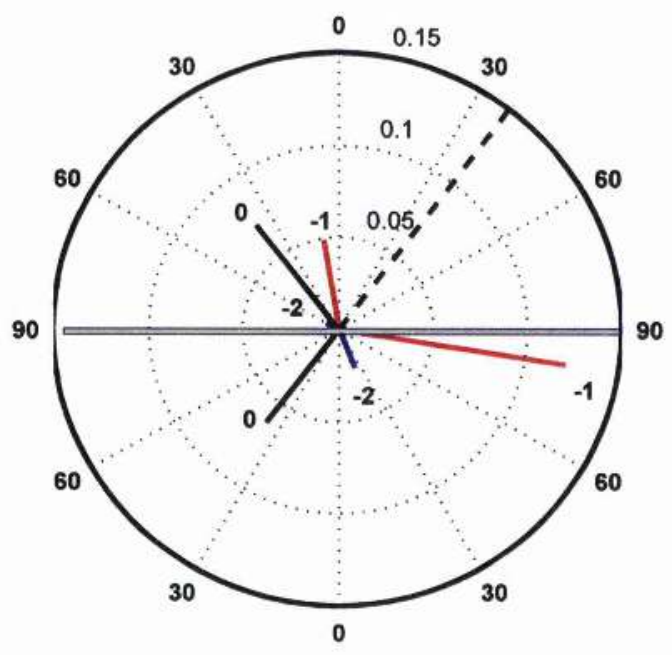

Figure 4-10: Transmitted and reflected modes for $\left(\theta=37^{\circ}, \phi=0^{\circ}\right.$ TE pol. $)$ at $2 \mathrm{GHz}$

\subsection{Reinforced Concrete Structures}

Reinforced concrete is used in various civil engineering applications such as the construction of structural frames, foundations, retaining walls, highways and bridges. It is a composite material consisting of steel reinforcing bars embedded in concrete, which takes advantage of the high compressive strength of concrete and the ability of steel to resist high tensile stresses. Since the steel bars which are embedded into the concrete usually follow a periodic pattern, similar frequency selective and scattering behaviour observed in masonry block structures walls is expected to be observed in reinforced concrete as well. They are normally designed according to recommendation given in British Standards (BS 5400, BS 8007 and BS 8110 ) based on the requirements in strength and tension. Figure 4-11 shows a typical concrete reinforcement with vertical steel bars. These bars present an $x$-axis periodicity which can be easily simulated with the developed RCWA model. For the model geometry shown in Figure 4-1 to apply, the steel bars circumference has been approximated with squares with side equal to the diameter of the steel bar. For the purpose of this investigation a reinforced concrete wall with thinkness $H=200 \mathrm{~mm}$, reinforced with $20 \mathrm{~mm}$ (diameter) steel bars placed vertically in an $\mathrm{x}$-axis 
periodic pattern with periodicity $d=15 \mathrm{~cm}$ is considered. Therefore, the RCWA model parameters are: $h_{2}=20 \mathrm{~mm}, h_{1}=h_{3}=\left(H-h_{2}\right), d_{1}=h_{2}, d_{2}=d-d_{1}$. The concrete dielectric permittivity was set to $\varepsilon_{1}=\varepsilon_{3}=\varepsilon_{22}=7+j 0.3$ and the one for steel to $\varepsilon_{22}=$ $-j \sigma / 2 \pi f \varepsilon_{o}$ where the $\sigma$ is the conductivity $\left(\sigma=1.11 \times 10^{6} \mathrm{~S} / \mathrm{m}\right) f$ is the frequency and $\varepsilon_{o}$ is the free space permittivity. 41 modes have been considered into the calculation of the reflection and transmission coefficients as suggested by the convergence study shown in Figure 4-12

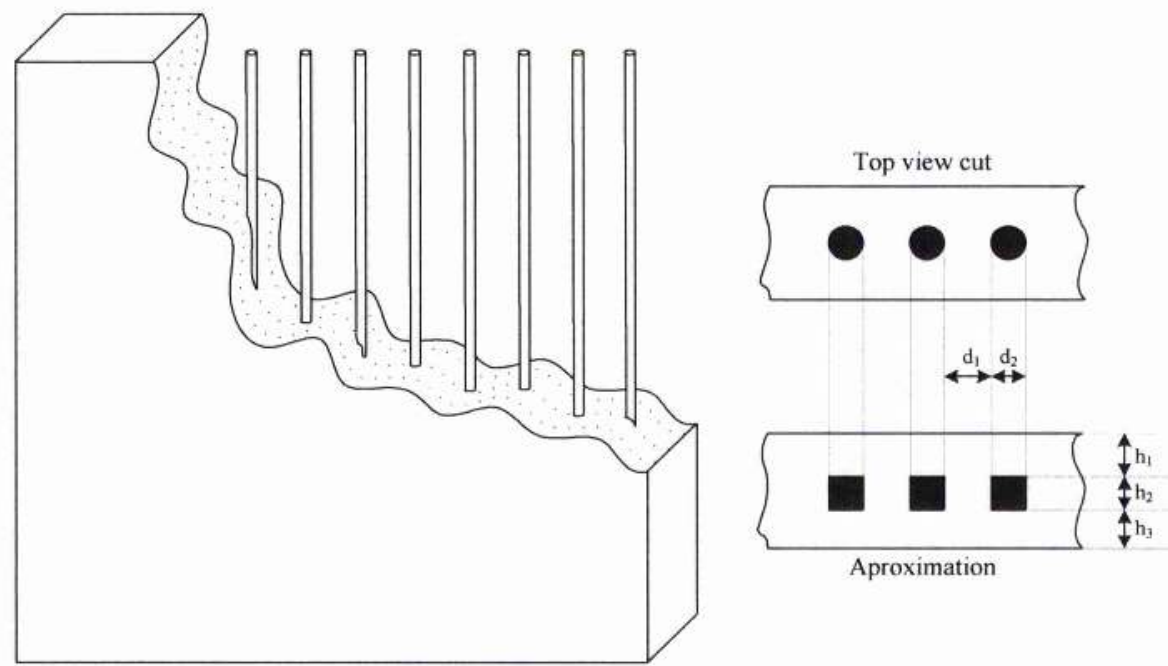

Figure 4-11: Reinforced Concrete

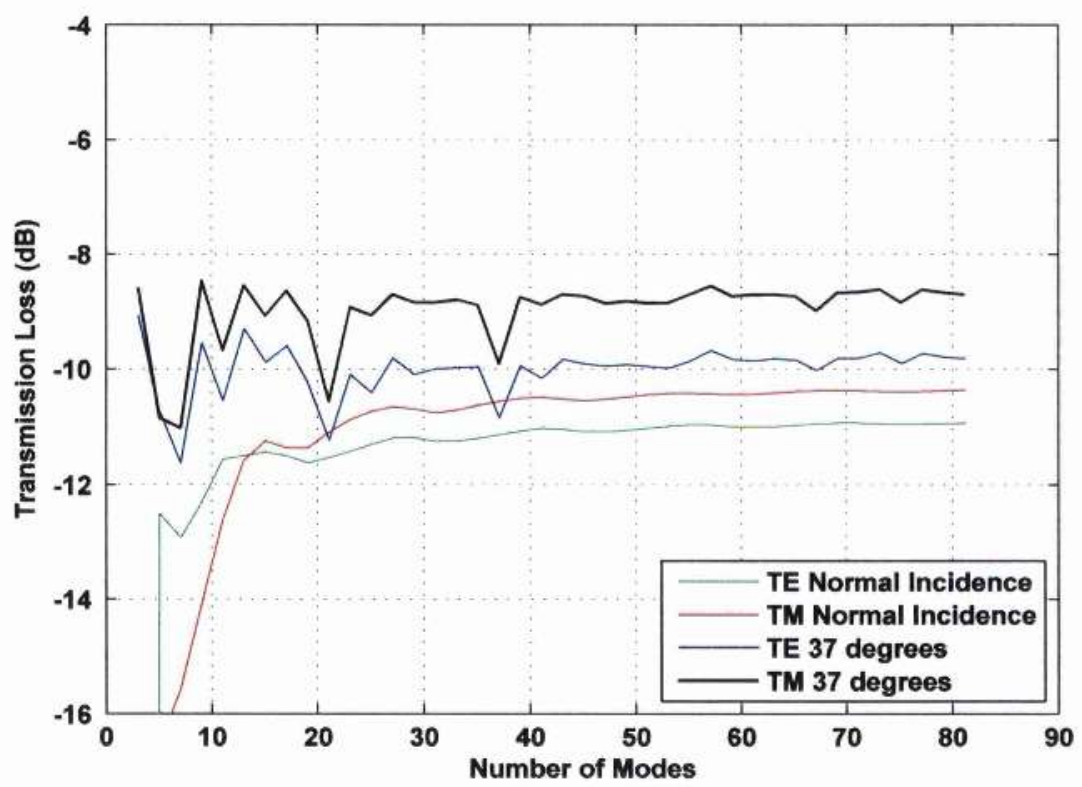

Figure 4-12: Convergence Study for a $20 \mathrm{~cm}$ thick reinforced concrete wall at $3 \mathrm{GHz}$ (steel bar periodicity $=15 \mathrm{~cm}$ ) 


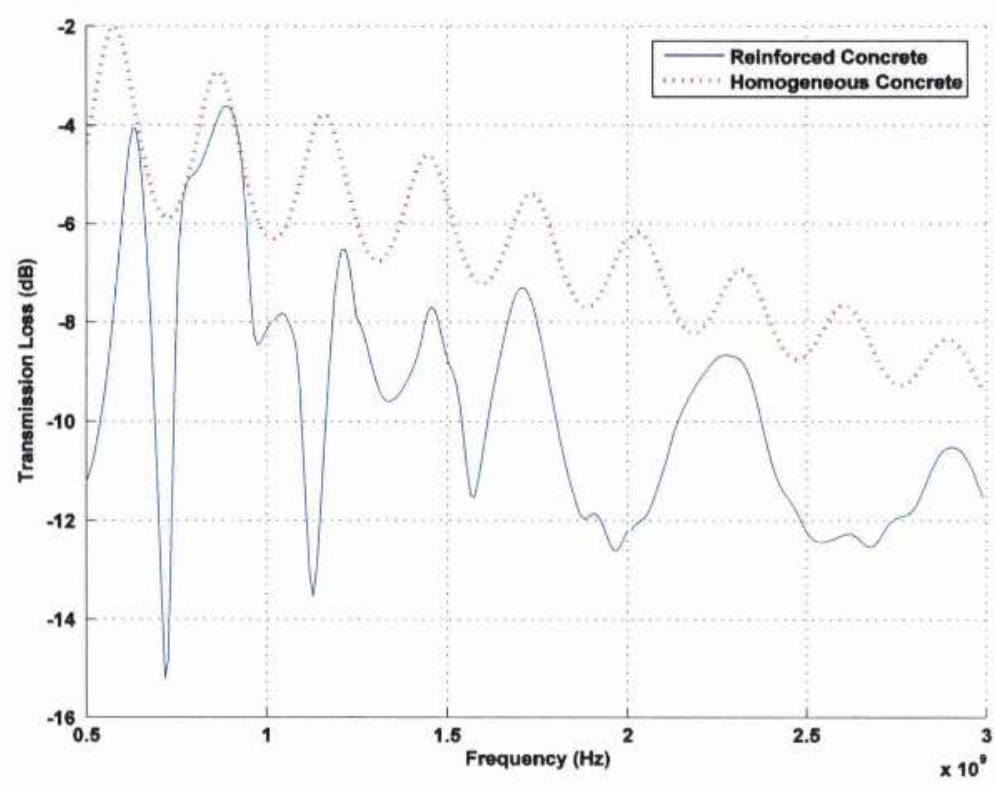

(a)

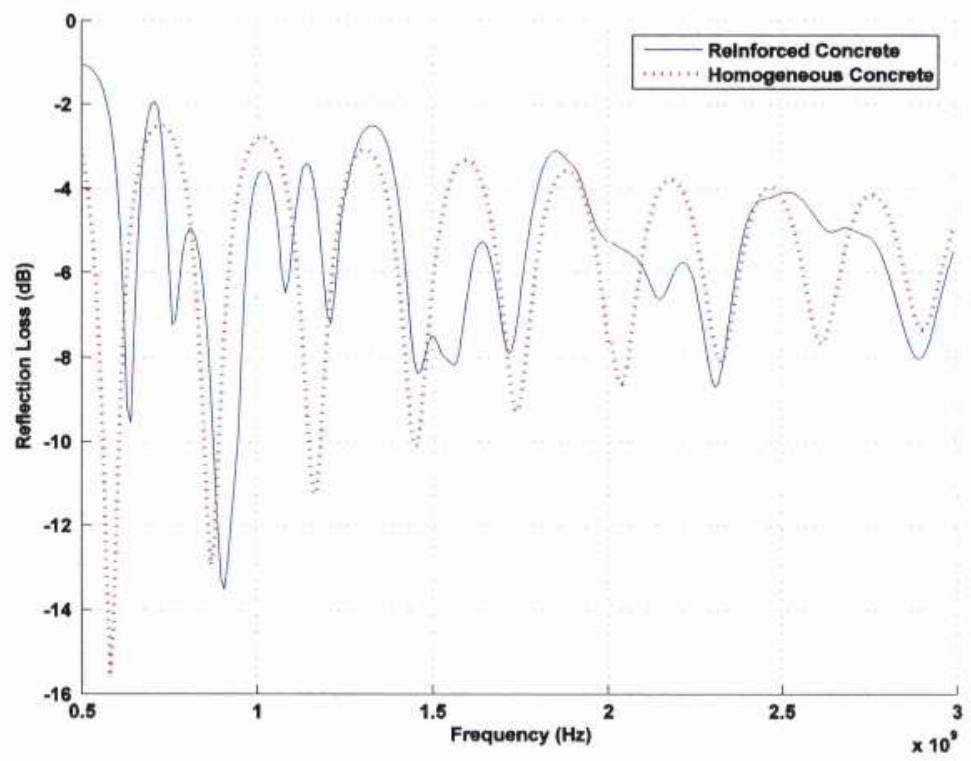

(b)

Figure 4-13: Transmission/Reflection Coefficients for a $20 \mathrm{~cm}$-thick Reinforced Concrete wall $\left(\theta=37^{\circ}\right.$,

$$
\phi=0^{\circ} \text {, TE pol.) }
$$

Figure 4-13 shows a comparison between the reflection/transmission characteristics of a $20 \mathrm{~cm}$ reinforced concrete wall and a homogeneous concrete wall for TE polarization under an oblique angle of incidence $\left(\theta=37^{\circ}, \phi=0^{\circ}\right)$. Similarly to the masonry case, resonances arise when the structure becomes periodic. This effect is again more significant on the transmission characteristics. 


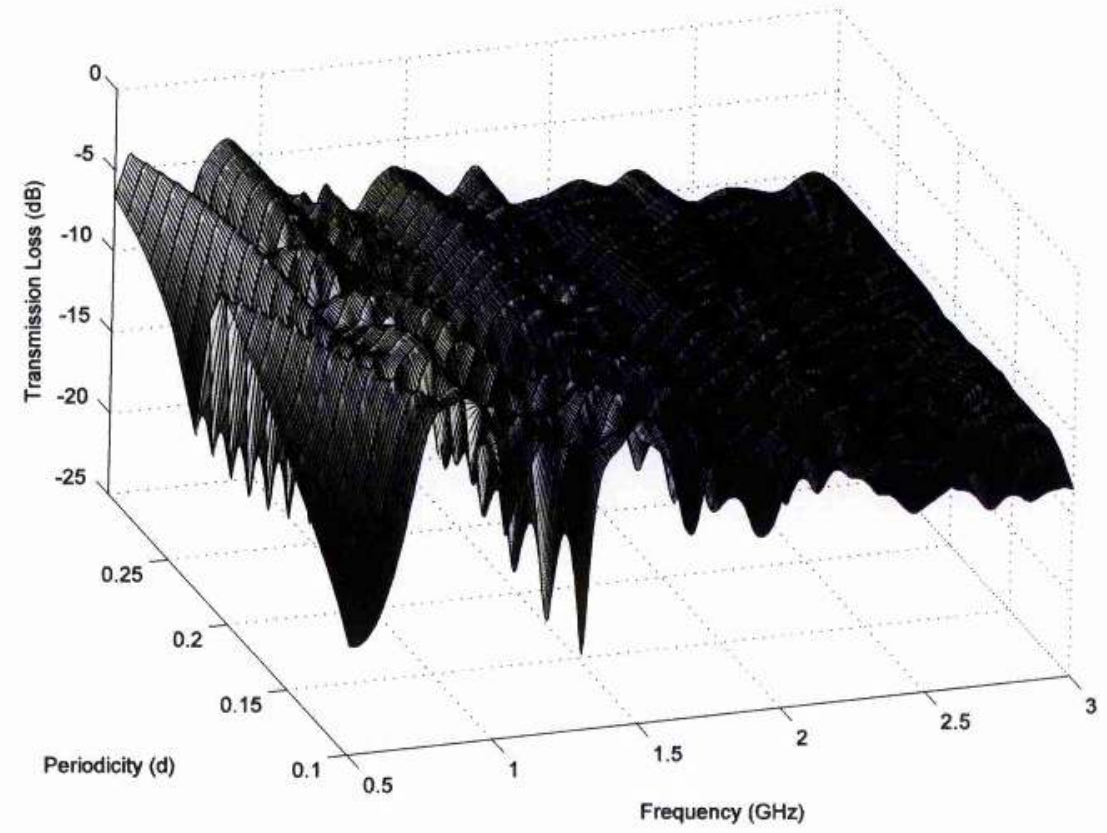

Figure 4-14: Effect of steel rod periodicity (d) on the transmission loss of a $20 \mathrm{~cm}$-thick Reinforced concrete $\left(\theta=37^{\circ}, \phi=0^{\circ}\right.$, TE pol. $)$

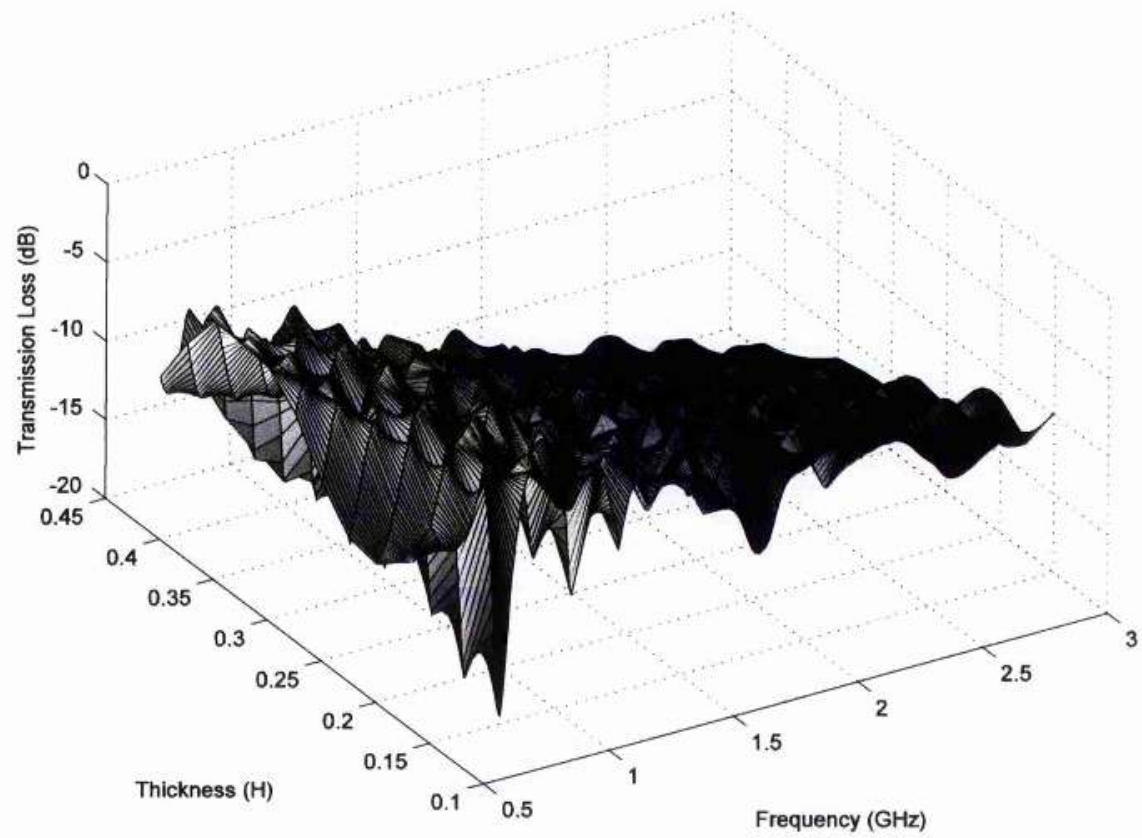

Figure 4-15: Effect of a reinforced concrete wall thickness $(\mathrm{H})$ on the transmission loss $\left(\theta=37^{\circ}, \phi=0^{\circ}\right.$, TE pol., $d=15 \mathrm{~cm}$ ) 
Figure 4-14 illustrates the effect of varying the periodicity of the steel bars for a $20 \mathrm{~cm}$ thick reinforced concrete wall on the transmission loss frequency response. It is observed that the resonances are slightly shifted in frequency making the interface to present different frequency selectivity on the incident waves for various periodicities. Similar behaviour is observed in Figure 4-15 which illustrates the effect of varying the reinforced concrete wall thickness for a fixed periodicity $(d=15 \mathrm{~cm})$.

As in the case of masonry block walls, as the frequency increases, higher modes can begin to propagate in the air regions in either side of a periodic structure. Figure 4-16 illustrates the transmission loss of various propagating modes as a function of frequency. For frequencies below $1.25 \mathrm{GHz}$ only the specular component propagates (mode 0 ). At $1.25 \mathrm{GHz}$ mode -1 is excited where as mode -2 starts to propagate at $2.5 \mathrm{GHz}$. Figure 4-17 demonstrates the angle of departure of these modes at various frequencies as soon as they start propagating. The minus sign on the angles means that the propagating mode propagates in the opposite $\mathrm{x}$-direction compared to the specular one. At $2 \mathrm{GHz}$ the mode -1 carries significant power and it propagates in a very different direction from the specular transmitted mode. This is shown in Figure 4-17. It can be also observed that at this frequency most of the reflected energy propagates along the specular reflection direction with mode -1 carries insignificant contributions.

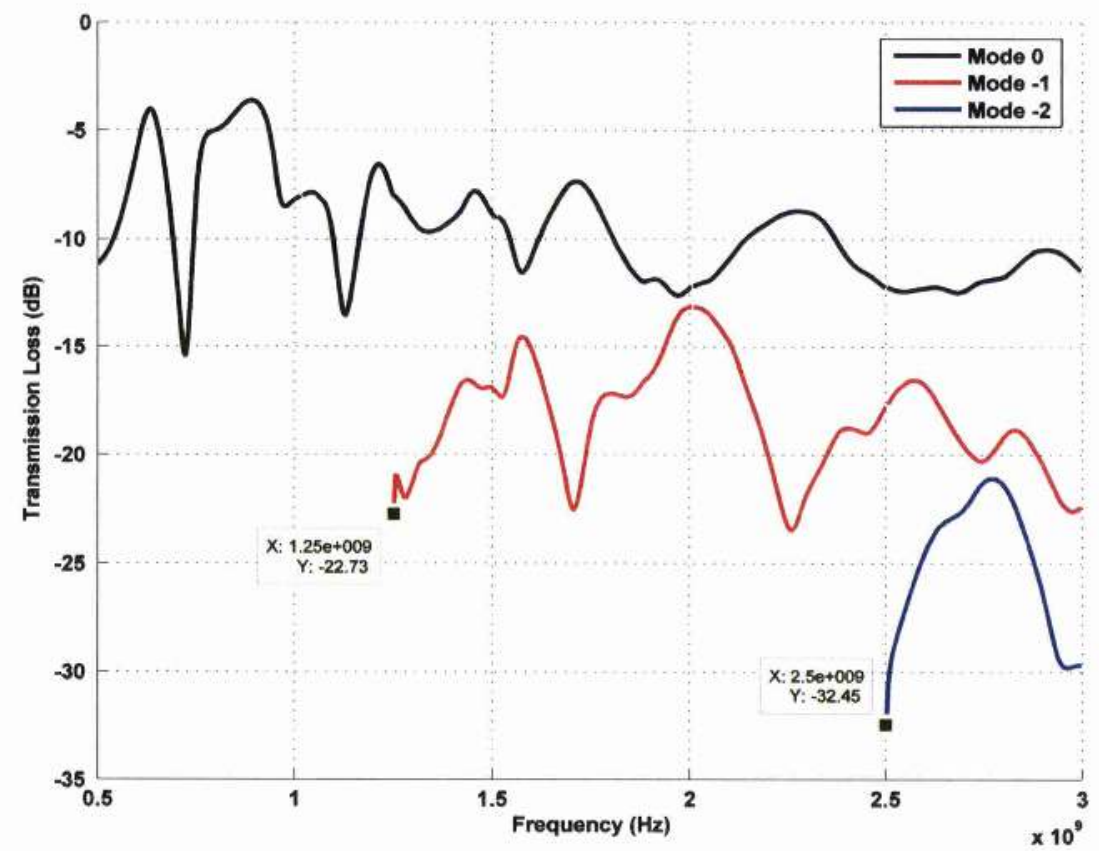

Figure 4-16: Transmission loss of various propagating modes, $\left(\theta=37^{\circ}, \phi=0^{\circ} \mathrm{TE}\right.$ pol. $)$ for a reinforced concrete wall 


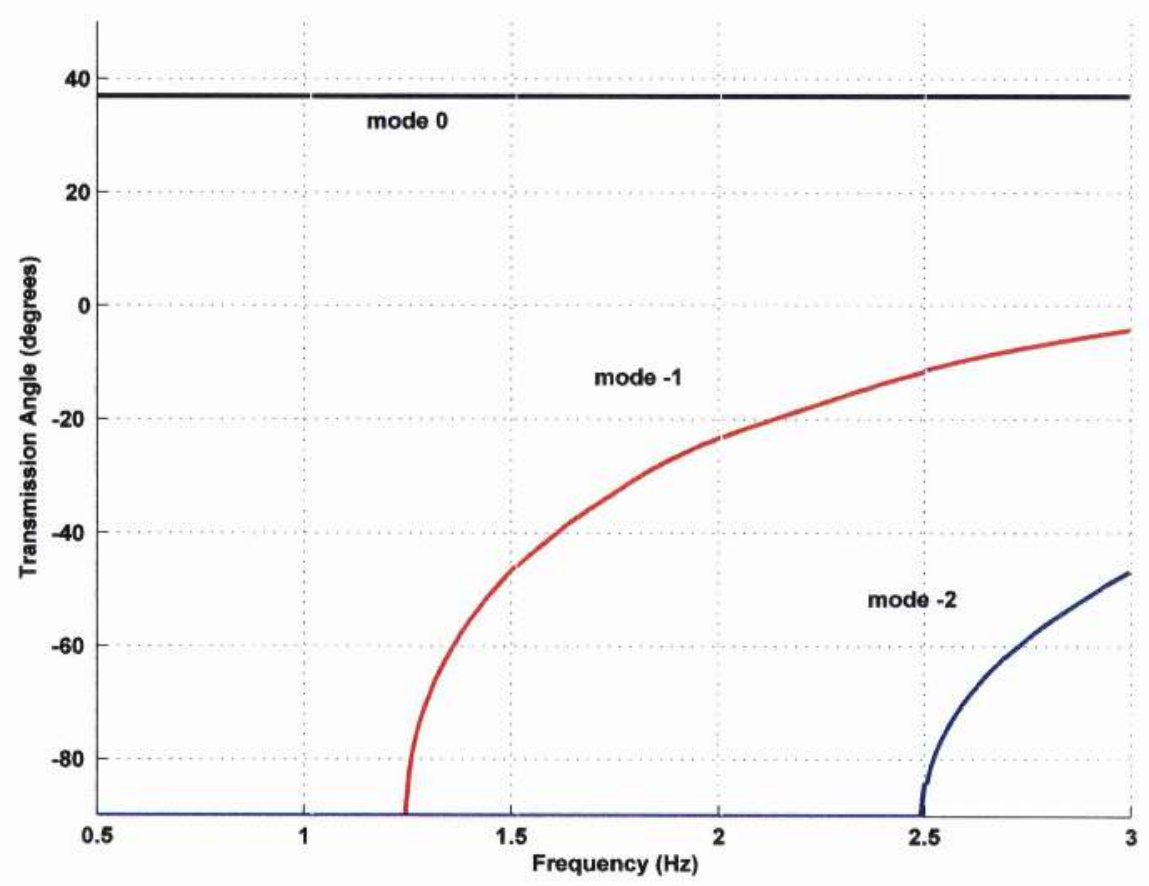

Figure 4-17: Transmission angles of various modes at various frequencies for a reinforced concrete wall

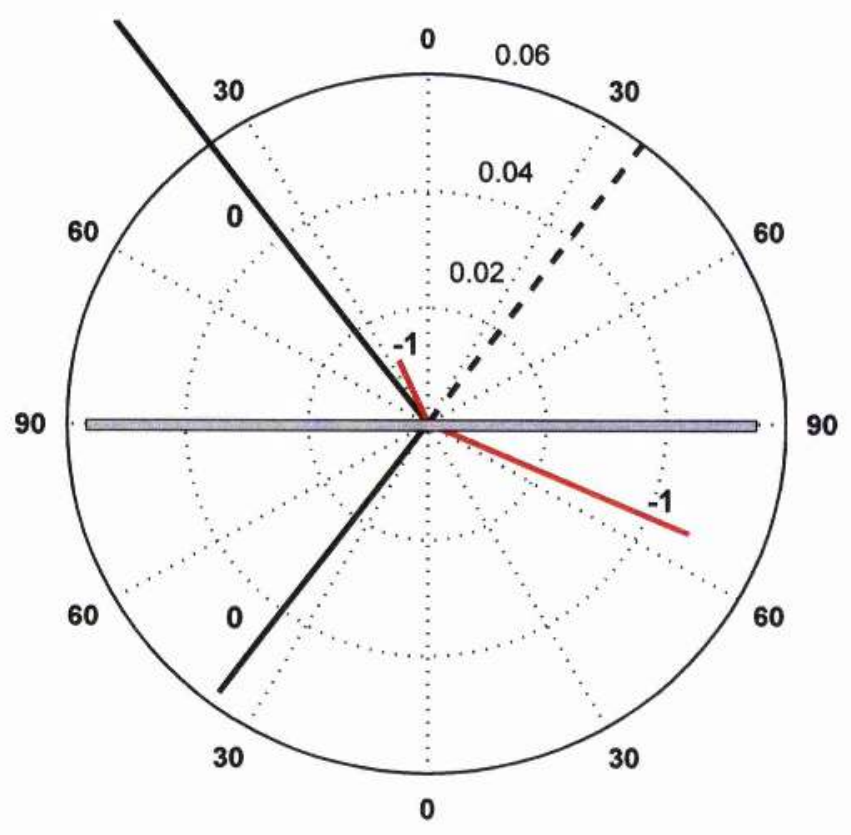

Figure 4-18: Transmitted and reflected modes for $\left(\theta=37^{\circ}, \phi=0^{\circ} \mathrm{TE}\right.$ pol. $)$ at $2 \mathrm{GHz}$ from a reinforced concrete wall 


\subsection{Summary}

The natural building behavior has been examined in this chapter through the use of a custom-written RCWA model. This model has been verified, by comparing it with results obtained from a commercial EM simulator; the CST Microwave Studio and has been used to examine the frequency selectivity and scattering behaviour of typical periodic structures such as the masonry block and reinforced concrete walls. It has been observed that such structures exhibit resonances in their transmission and reflection characteristics which seem to depend on the angle of incidence, the periodicity of the structure, the wall thickness and the constitutive parameters of the walls' materials. They also give rise to the propagation of various higher order modes (harmonics) in various angles of departure, which sometimes can carry more energy than the specular ones.

Low correlation scattering behaviour can be useful in single-input multiple-output (SIMO) systems, in which the presence of various copies of the transmitted signal arriving at the receiver with various phases and amplitudes is beneficial. Low correlation between the received rays enables this kind of systems to achieve high capacities. However, the utilisation of this scattering behaviour is beyond the scope of this research.

The frequency selectivity exhibited by building structures has raised the idea of transforming the building into a frequency selective one, where signals of specific frequencies are not allowed to enter and/or escape the building. However, since the internal structure and parameters of building periodic interfaces are usually unknown, it would be more or less impossible to utilise this natural frequency selectivity. A way to easily transform building interfaces into frequency selective, tuneable at a desired frequency range, is to deploy surfaces on those interfaces which have frequency selective characteristics. These surfaces are called frequency selective surfaces whose principles and deployment on building walls are studied in the next chapter. 


\section{Chapter 5}

\section{Frequency Selective Surfaces}

Frequency Selective Surfaces (FSS) are planar periodic structures, which consist of identical thin conducting elements, usually printed on dielectric substrates (see Figure 5-1) for support. They behave as electromagnetic filters (passive or active), selectively reflecting or attenuating a desired frequency band. This selectivity emerges from the periodicity that the conducting elements have between each other. This can be readily understood by inspecting the equivalent circuit of a simple periodic structure. Figure 5-2 presents the equivalent circuits of a periodic surface of infinitely long rods and a periodic surface of finite conducting elements [56]. The infinitely long rods result in an inductive circuit which tunes only at DC, where as the gaps in the finite elements result in series capacitors, which together with the inductors form a series $L C$ circuit exhibiting a resonant frequency $f_{o}$.

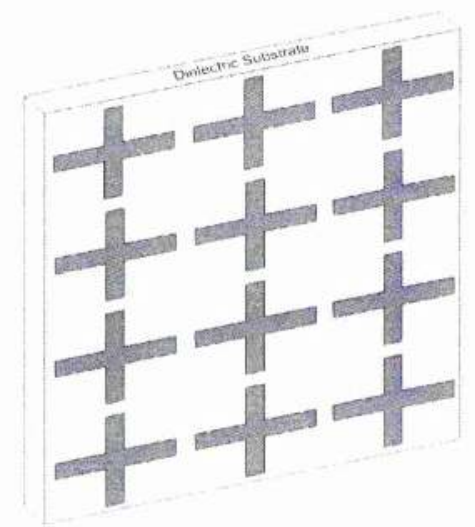

Figure 5-1: Typical Frequency Selective Surface (Cross Dipole Design)

This chapter deals with the basic principles governing the operation and analysis of FSS. It also presents, through simulations and anechoic chamber measurements the interaction of FSS with typical building materials. This interaction plays an important role in the design of frequency selective wireless environments through the use of Frequency Selective Surfaces. 


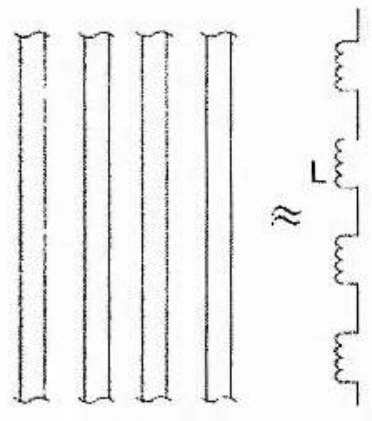

(a)

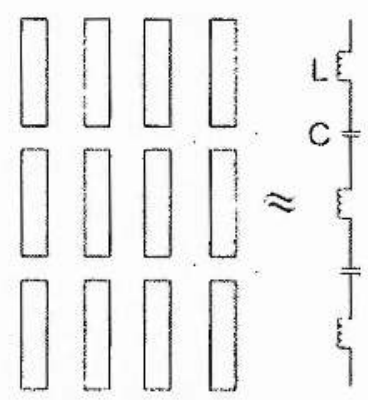

(b)

Figure 5-2: Equivalent Circuits of (a) a periodic surface of infinitely long rods and (b) a periodic surface of finite conducting elements

\subsection{Basic FSS characteristics}

\subsubsection{Band-pass and Band-stop operation}

FSS can act as band-pass and band-stop filters. Figure 5-3 presents a typical example of a FSS that has this behaviour. In this example, the band-stop FSS is made of an array of circular loops where as the band-pass is made of an array of slot circular loops. This is a simple case of the general Babinet Principle of complementary arrays [57]. When the two FSS arrays are placed on top of each other, a complete perfectly conducting plane is obtained. Based on this principle, if the conducting plane is perfectly conducting and infinitely thin, then the reflection coefficient of the one array equals to the transmission coefficient of the other. It has to be noted that, for dielectric thicknesses greater than $\lambda / 4$ this principle does not stand.

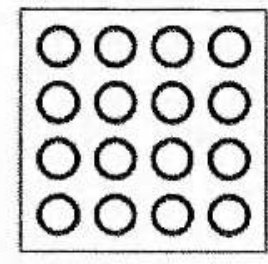

Band-Stop

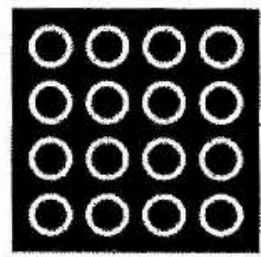

Band-Pass

Figure 5-3: Complementary arrays of Circular Loop FSS

\subsubsection{Active FSS}

So far the discussion was about passive FSS. However, active FSS have also been proposed in literature [58]. In this case the frequency properties can be varied by either using semiconductor devices (i.e. PIN diodes) placed into the elements [59]-[60], or by printing the elements on substrates whose dielectric properties can be varied [61]. A typical example could be 
the switching of a totally reflecting surface (at a particular frequency) to totally transparent one. Figure 5-4 shows an active square loop FSS [62]. When the diodes are forward biased (ON) a resistive load is added between the half squares forcing the structure to act as a normal square loop FSS. On the other hand, when the diodes are reversed biased (OFF), a gap is inserted between the half squares, which acts as a capacitive component in series, changing the FSS resonant frequency. The CFDTD (Conformal Finite Difference Time Domain) simulation results verify that by switching ON and OFF the diodes the FSS becomes totally reflecting or totally transparent at $13 \mathrm{GHz}$ respectively. The other way to make the FSS active is to make the supporting substrate active. As it will be discussed later on, the dielectric properties of the substrate have significant impact on the tuning frequency of the FSS. The authors of [63] have suggested ferrite substrate, whose permeability changes with DC magnetic bias. Also, the authors of [64] have proposed a substrate whose effective permittivity changes by exchanging the dielectric liquids within it.
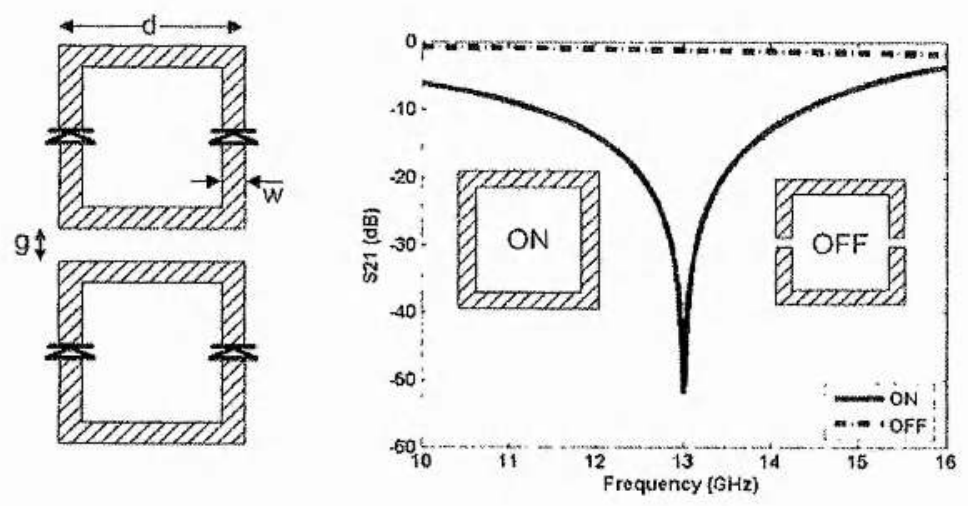

Figure 5-4: Active Square Loop FSS with PIN diodes $(d=7 \mathrm{~mm}, g=3.2 \mathrm{~mm}, w=1 \mathrm{~mm}$ on FR4 dielectric with $\varepsilon_{r}=4.55$ )

\subsubsection{The supporting dielectric}

As already mentioned, a FSS includes a dielectric substrate, which is used for mechanical support. This substrate has significant effect on the transmission and reflection response of the FSS [65]. Luebbers and Munk [66] have investigated the effect of cladding an array of rectangular slots in dielectric slabs. They have noted a shift of the resonant frequency by approximately the square root of the dielectric constant. They havebrb

also noted that the surface waves can be 'trapped' into the substrate giving rising to additional resonances. The authors of [67]-[69] have investigated the decrease of the resonant frequency by increasing the dielectric substrate thickness. Their results suggest that there exists a rapid decrease of the resonant frequency as the dielectric thickness is increased from zero to $\lambda / 8$. They also noted that the resonant frequency converges to an asymptotic value which differs 
amongst the different authors. Figure 5-5 presents a CFDTD simulation of the substrate $\left(\varepsilon_{\mathrm{r}}=2.775\right)$ thickness as a function of the resonant frequency of a cross-dipole FSS. The result verifies that there is a rapid change of the resonant frequency for thicknesses up to $\lambda / 8$. The resonant frequency seems to converge for thicknesses beyond $\lambda / 4$ to a value equal to $\frac{f_{o}}{\sqrt{\left(\varepsilon_{r}+1\right) / 2}}$ where $f_{o}$ is the resonant frequency of the FSS without the substrate. Likewise when the cross-dipole element is enclosed in two dielectric substrates, the resonant frequency decays rapidly up to $\lambda / 8$ and converges to $\frac{f_{o}}{\sqrt{\varepsilon_{r}}}$ for thickness beyond $\lambda / 4$ as Luebbers and Munk suggest in [66].

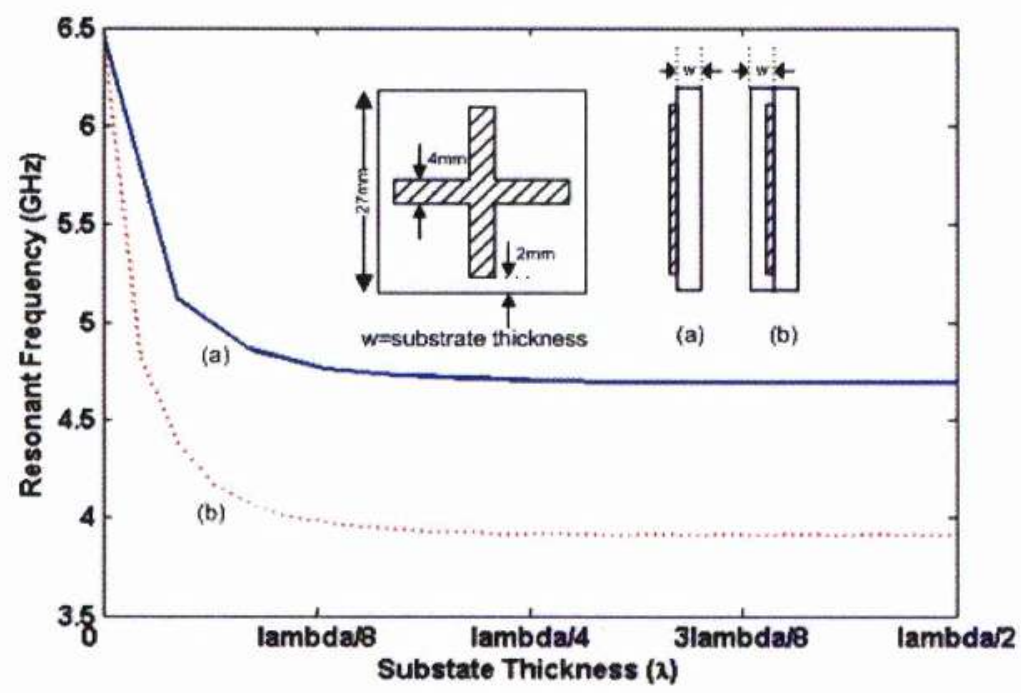

Figure 5-5: Effect of the dielectric thickness on a cross dipole FSS. (a) Dielectric only on one side (b) dielectric on both sides of the elements

\subsubsection{Element Types}

Another important factor that needs to be considered when designing a FSS is the selection of the element geometry. Some element shapes have a more broadband response than others; some are more sensitive to the angle of incidence, whereas some are more suited for cross-polarised waves. Figure 5-6 presents a basic selection of the most commonly studied element geometries. Table 5-1 presented by $\mathrm{Wu}$ in [70] presents a typical comparison between these different elements. 


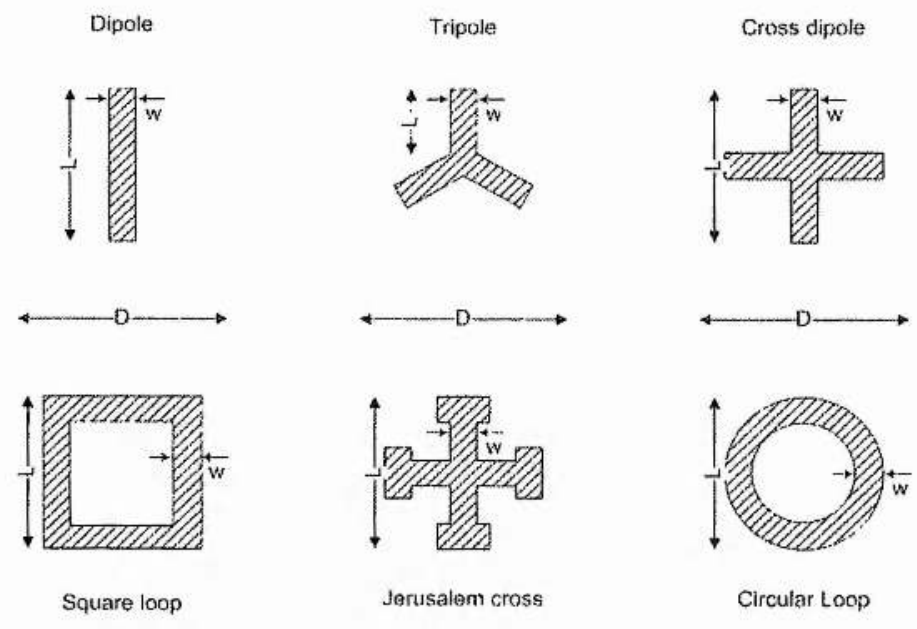

Figure 5-6: Unit cells of the basic FSS element geometries

\begin{tabular}{|c|c|c|c|c|}
\hline $\begin{array}{c}\text { Element } \\
\text { Type }\end{array}$ & $\begin{array}{c}\text { Angular } \\
\text { Insensitivity }\end{array}$ & $\begin{array}{c}\text { Cross- } \\
\text { Polarization }\end{array}$ & $\begin{array}{c}\text { Larger } \\
\text { Bandwidth }\end{array}$ & $\begin{array}{c}\text { Small Band } \\
\text { Separation }\end{array}$ \\
\hline Jerusalem Cross & 2 & 3 & 2 & 2 \\
\hline Rings & 1 & 2 & 1 & 1 \\
\hline Tripole & 3 & 3 & 3 & 2 \\
\hline Cross dipole & 3 & 3 & 3 & 3 \\
\hline Square Loop & 1 & 1 & 1 & 1 \\
\hline Dipole & 4 & 1 & 4 & 1 \\
\hline
\end{tabular}

Ratings: $1=$ best, 2 =second best, etc.

Table 5-1: Performance of various element geometries

\subsubsection{Brief history and applications}

FSS applications have been proposed in literature including but not limited to the modification of the characteristics of antenna systems. For example FSS sub-reflectors can be used in dual-reflector antenna configurations. These sub-reflectors are mainly used to enhance the multi-frequency capabilities of such antenna systems. Figure 5-7 shows the Cassegrain Antenna System [71] [72] where an FSS sub-reflector is placed in front of the main reflector. This subreflector is transparent at a frequency $f_{1}$ and opaque at a frequency $f_{2}$. This enables the use of two horn antennas; one antenna placed at the focal point of the main reflector transmitting $f_{l}$ and the other one placed at the Cassegrain focal point transmitting $f_{2}$. The net result is that two frequencies $\left(f_{1}\right.$ and $\left.f_{2}\right)$ can be accommodated at the main-reflector. 
FSS are exploited also in military applications. A typical example is the radome in the nose of an air-fighter. In this case the radome has to ensure a total reflection of all frequencies expect of the operation frequency band of the radar.

FSS have also been used as polarization transformers in antenna systems that rotate the polarization of a linearly polarized wave or convert the linear polarization into circular [73][74][75]. For example if a linearly polarized wave is incident on the FSS, it can be decomposed into vertical and horizontal components where the one is delayed and the other one is advanced in phase. If the phase difference between the two components is $90^{\circ}$ then the two components will recombine into a circularly polarized wave.

Another application includes antennas that are mounted on a mast. The entire assembly is covered with FSS which behave as band-stop filters in order to deflect out of band signal and allow desired signals to go through. This ensures that low Radar Cross Section (RCS) is obtained.

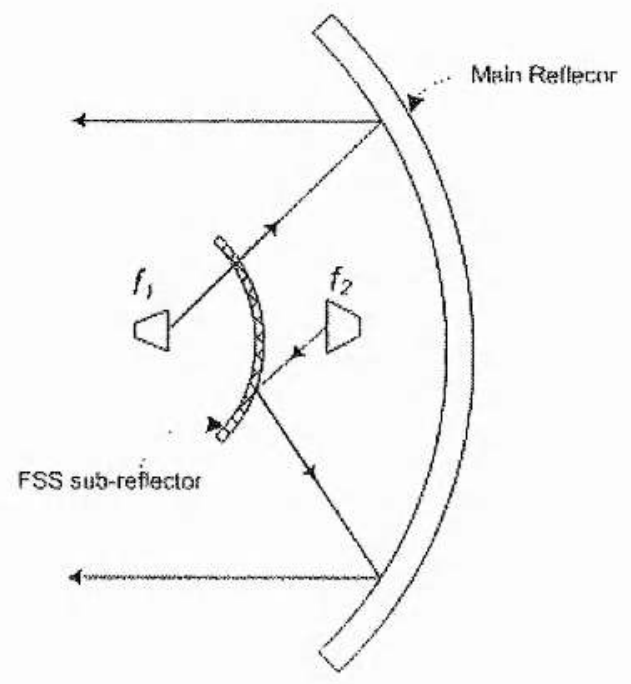

Figure 5-7: Use of FSS as a Cassegrain Sub-reflector

Apart from applications in antenna design, FSS have found use in other areas. An example is their application in circuit analog absorbers [56], [76]. The reflectivity/bandwidth of conventional microwave absorbers, such us the Salisbury Screen, can be enhanced by adding a reactive component on their resistive layer. By incorporating a FSS into the layered structure of the absorber this reactive impedance component can be achieved. This ensures that larger bandwidths can be achieved especially if more FSS layers are added. 


\subsection{Techniques for analyzing Frequency Selective Surfaces}

Many approaches have been proposed in literature for analyzing and designing frequency selective surfaces [77]. Some of the more important are, the Periodic Moment Method (PMM) [56],[78] the Finite Element Method (FEM) [79][80], the finite-difference time-domain (FDTD) method [81][82] and its conformal version (CFDTD) [83] [84], the Spectral Domain Method (SDM) [85] [86], the recursive T-matrix Algorithm (RTMA) [87] [88], and the equivalent circuit method (ECM) [89].

\subsubsection{Periodic Moment Method (PMM)}

The Periodic Moment Method was developed by Ben Munk [56] and his colleagues [78] in Ohio State University and serves as one of the more powerful numerical analysis techniques in FSS. In this method, the mutual impedance between the array elements and an arbitrary selected element are evaluated using the plane wave expansion. Using the induced voltages (by an incident plane wave) on the reference element, the terminal current on this element can be derived using the mutual impedance approach. Assuming that we know the shape of this current, then the current on the whole structure can be known as well and the scattered field can be easily calculated. However, the mutual impedance approach has been considered as inaccurate. The problem arises from the fact that in general we do not know very well the current distributions along this element. In order to overcome this problem the elements are split into mathematical segments, which are so small so that the current distribution on each segment is not so significant. More details and complete formulation for this method can be found in [56] and [78].

\subsubsection{Finite Element method (FEM)}

The Finite Element Method is a frequency domain computational method for electromagnetic simulations. However, it was originated from the need of solving civil and mechanical engineering problems. It combines the geometrical adaptability and the material generality for solving arbitrary and very complex shapes of any composition. The latter is of significant importance in electromagnetics since many applications require simulation of nonmetallic/composite structures. Mathematically, is used for finding approximate solutions to partial differential (PDE) equations and integral equations (IE). The computational domain is broken down into finite elements of simple shapes. Suitable interpolation polynomials (also known as basis or shape functions) are used to approximate the unknown function within each element. These shape functions can be node-based or edge-based. In the node-based elements, the form of the shape function at each element is controlled by the function values at its nodes. Since in electromagnetic simulations, the vector electric and magnetic fields need to be represented, node- 
based elements impose serious problems. Firstly, spurious modes are observed when modelling cavity structures. Node-based functions impose continuity in all three spatial components. On the other hand edge-based functions guarantee continuity only along the tangential component. This feature of the edge-based functions mimics the behaviour of field components along discontinuous boundaries and they are extensively used to solve complex electromagnetic problems. More information on FEM and its application in FSS can be found in [79] and [80].

\subsubsection{Finite-Difference Time-Domain method (FDTD)}

The FDTD method was firstly introduced by Yee [92] in 1966. Since then, it has been widely used in the analysis of electromagnetic scattering, radiation and propagation problems. This method does not rely on the solution of the traditional complex frequency domain techniques. Yee has replaced the original Maxwell equations with a set of finite difference equations. These equations are solved in a "leapfrog" manner, i.e. the electric field equations are solved in one instance in time where as the magnetic field ones are solved in the next instance in time. This is done because if we examine the Maxwell equations, we can observe that the change in the E-field in time is depended on the change of the $\mathrm{H}$-field across the space (curl). This results in the basic FDTD time-stepping relation, the updated value of the E-field in time, depends on the stored value of the E-field and the numerical curl of the local distribution of the $\mathrm{H}$-field in space. Similarly the H-field is time-stepped as well; the updated value of the H-field depends on the stored value of the $\mathrm{H}$-field and the numerical curl of the local distribution of the E-field in space. In order to account for multidimensional computations of the field, the computational domain is subdivided by using an orthogonal mesh in the Cartesian coordinate system. The vector components of the $\mathrm{E}$ and $\mathrm{H}$-fields are arranged on these unit cells in such a way so that an $\mathrm{E}$-field vector component always lies between two $\mathrm{H}$-field vector components, and vice versa. This scheme is known as Yee Lattice and is shown in Figure 5-8. The E-components are in the middle of the edges and the H-components are in the centres of the faces.

Since the FDTD method requires computation of the field components for all the points in the computation domain, this domain needs to be made finite in order to minimise the computer memory requirements of the simulation. This is achieved by adding artificial radiation or absorbing boundaries (ABC) into the computation domain [93]. For the case that the structures of interest have periodicity in one or two dimensions, such as Frequency Selective Surfaces, periodic boundary conditions $(\mathrm{PBC})$ should be used. For such cases the periodic structures are truncated into single-period structures with $\mathrm{PBC}$ in one or two dimensions [82]. The other sides of the computational domain must be truncated with $\mathrm{ABC}$ to avoid reflections. 


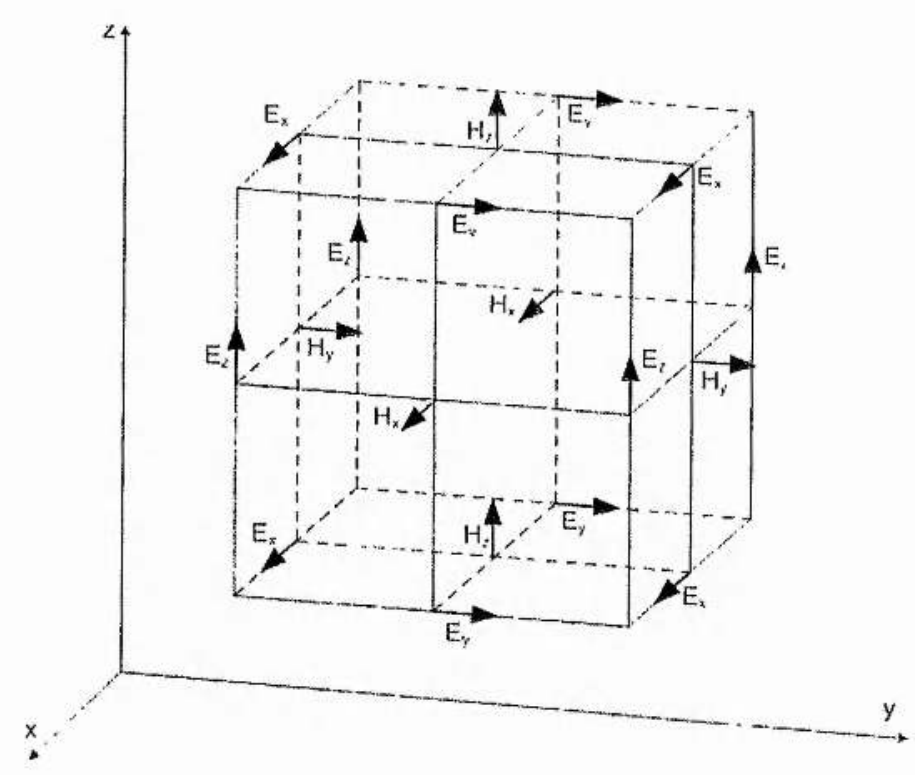

Figure 5-8: Yee Lattice.

\subsubsection{Conformal Finite-Difference Time-Domain Method (CFDTD)}

As already mentioned the original Yee-Lattice is based on the subdivision of the computational domain using an orthogonal mesh in the Cartesian coordinate system. The problem that arises from this is that it does not produce accurate results for curved edges or surfaces unless the mesh is made very fine to mitigate the error due to the stair-casing approximation. This however, would require more and heavier computation. The CFDTD techniques offer an alternative to fine discretization by using a more complex mesh generation to better describe curved surfaces without resorting to stair-casing. More information on the CFDTD method can be found in [83], [84].

\subsubsection{Spectral Domain Method (SDM)}

The basic idea of the Spectral Domain Method is to transform the partial differential equations (PDE) into the spectral domain. It uses Fourier Transform to formulate the tangential components of spatial domain scattered field from scatterers in the reference unit cells, as the Fourier integral of the tangential spectral domain scattered field. In this domain the PDEs can be reduced by one or more dimensions into an ordinary differential equation which can be easily solved using elementary functions. By using the Floquet theorem, these tangential components of the scattered field from the FSS are obtained by converting the Fourier integral into a summation of Floquet modes. One of the most important disadvantages of the SDM is that it cannot handle normal incidence. This happens because the tangential incident electric field vanishes at normal 
incidence. More information and complete formulation of the SDM and its application for FSS can be found in [85], [86].

\subsubsection{Recursive T-Matrix Algorithm (RTMA)}

The RTMA method [87], [88] does not require an absorbing boundary condition (ABC) like the conventional Finite-Difference techniques discussed above. Also, the discretization of the space is replaced by harmonic expansions of the fields and hence the number of unknown is significantly reduced. These methods are based on the tessellation of electrically large objects into small sub-scatterers whose individual transition matrices ( $\mathrm{T}$-matrices) can be represented by using low-order harmonic expansions. Once this is done, a recursive formula is used to sum up the effects of all the sub-scatterers to compute the fields.

\subsubsection{Equivalent Circuit Method (ECM)}

Despite the fact that Equivalent Circuit Method (ECM) can only be used for linear polarisation, and it is limited to certain element shapes, it is often a preferred modelling technique due to its implementation simplicity and quick evaluation. ECM is usually used as the initial tool in FSS design followed by any of the aforementioned electromagnetic simulation methods, to fine-tune the initial design or to simulate more complex structures. This method relies on the mapping of the FSS to its equivalent circuit which comprises of inductive and capacitive components. A typical example for a square loop FSS [89] is shown in Figure 5-9. The square loop FSS is broken down into vertical and horizontal strips. For TE incident wave the vertical strips contribute to the inductive component $\left(\mathrm{X}_{\mathrm{L}} / \mathrm{Z}_{\mathrm{O}}\right)$ and the horizontal strips contribute to the capacitive component $\left(\mathrm{B}_{\mathrm{c}} / \mathrm{Z}_{\mathrm{o}}\right)$. The capacitive component (Eq. 5.1) is multiplied by the effective permittivity $\left(\varepsilon_{\text {eff }}\right)$ of the dielectric substrate which supports the elements. According to [56] and [89], if one side of the FSS is backed by a dielectric substrate which has a thickness greater than $\lambda / 4$, the dielectric substrate will have $\varepsilon_{e f f}=\left(\varepsilon_{r}+1\right) / 2$. Equations 5.1-5.7 can be used for the calculation of the reflection and transmission coefficients for a square loop FSS. In these equations $\lambda$ represents the wavelength in air at the operating frequency and $\theta$ is the angle of incidence. It has to be noted that this approximation is only valid if $p(\sin \theta+1) / \lambda<1$ [90]. The square loop FSS formulation presented here was initially presented by Langley in [89] using the waveguide theory in [90]. However the initial formulation suffered from problems with varying angles of incidence. Sung in his paper [91] has added the $\sec (\theta)$ term in equations 5.1 and 5.2 to consider the effects of the varying angle of incidence. His simulation results had a very good agreement with measurements up an angle of 60 degrees. 


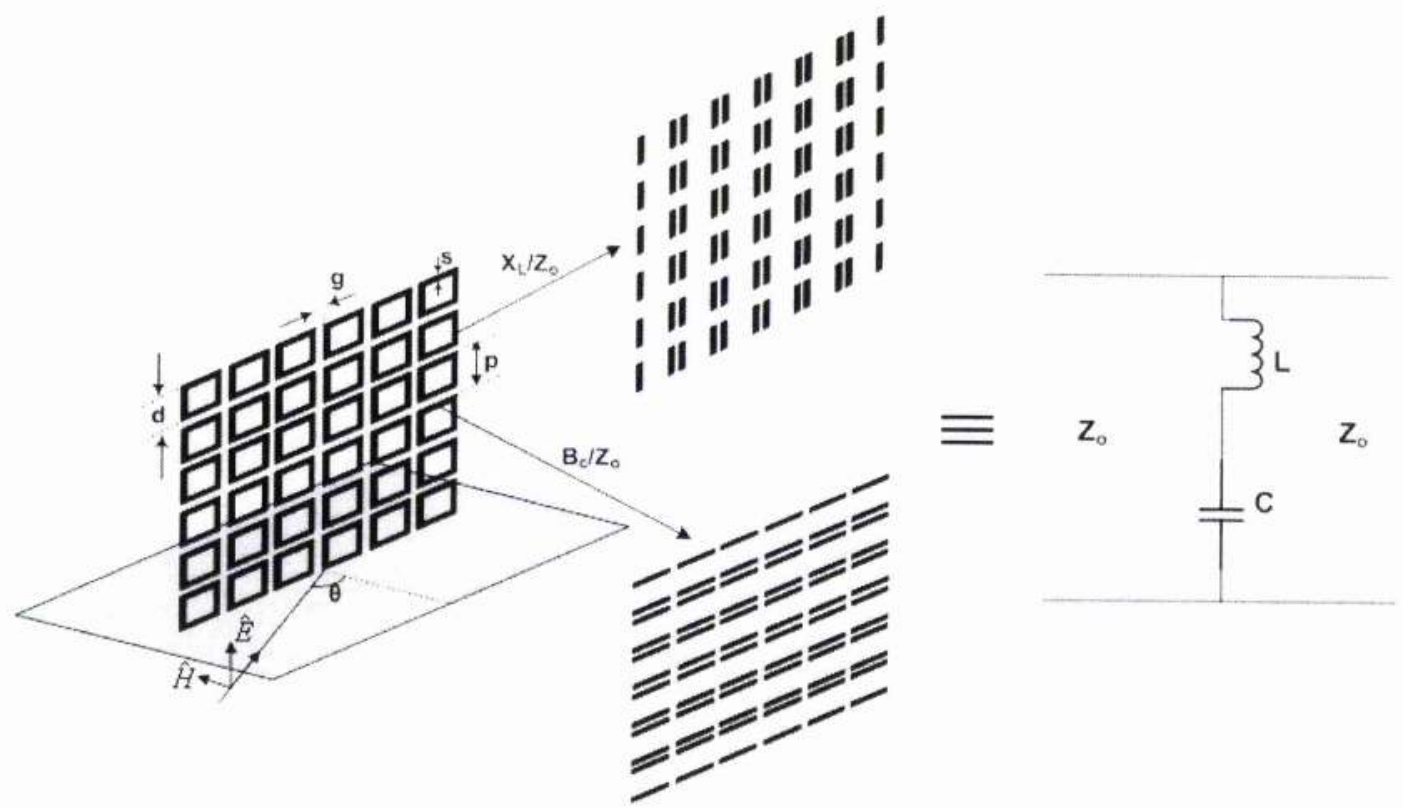

Figure 5-9: Equivalent Circuit Model of a square loop FSS

$$
\begin{gathered}
\frac{X_{L}}{Z_{o}}=\omega L=\frac{d}{p} \sec (\theta) F(p, 2 s, \lambda, \theta) \\
\frac{B_{C}}{Z_{o}}=\omega C=4 \frac{d}{p} \sec (\theta) F(p, g, \lambda, \theta) \varepsilon_{\text {eff }} \\
F(p, w, \lambda, \theta)=\frac{p}{\lambda} \cos \theta\left[\ln \left(\operatorname{cosec} \frac{\pi w}{2 p}\right)+G(p, w, \lambda, \theta)\right] \\
G(p, w, \lambda, \theta)=\frac{0.5\left(1-\beta^{2}\right)\left[\left(1-\frac{\beta^{2}}{4}\right)\left(A_{+}+A_{-}\right)+4 \beta^{2} A_{+} A_{-}\right]}{\left(1+\frac{\beta^{2}}{4}\right)+\beta^{2}\left(1+\frac{\beta^{2}}{2}-\frac{\beta^{4}}{8}\right)\left(A_{+}+A_{-}\right)+2 \beta^{6} A_{+} A_{-}} \\
A_{ \pm}=\frac{1}{\sqrt{\left[1 \pm \frac{2 p \sin \theta}{\lambda}-\left(\frac{p \cos \theta}{\lambda}\right)^{2}\right]}-1} \\
\beta=\sin \left(\frac{\pi w}{2 p}\right) \\
R=\frac{Z_{n}-1}{Z_{n}+1} \text { and } T=1-|R|^{2} \\
Z_{n}=j \times\left(\frac{X_{L}}{Z_{o}}-\frac{1}{\frac{B_{C}}{Z_{o}}}\right)
\end{gathered}
$$




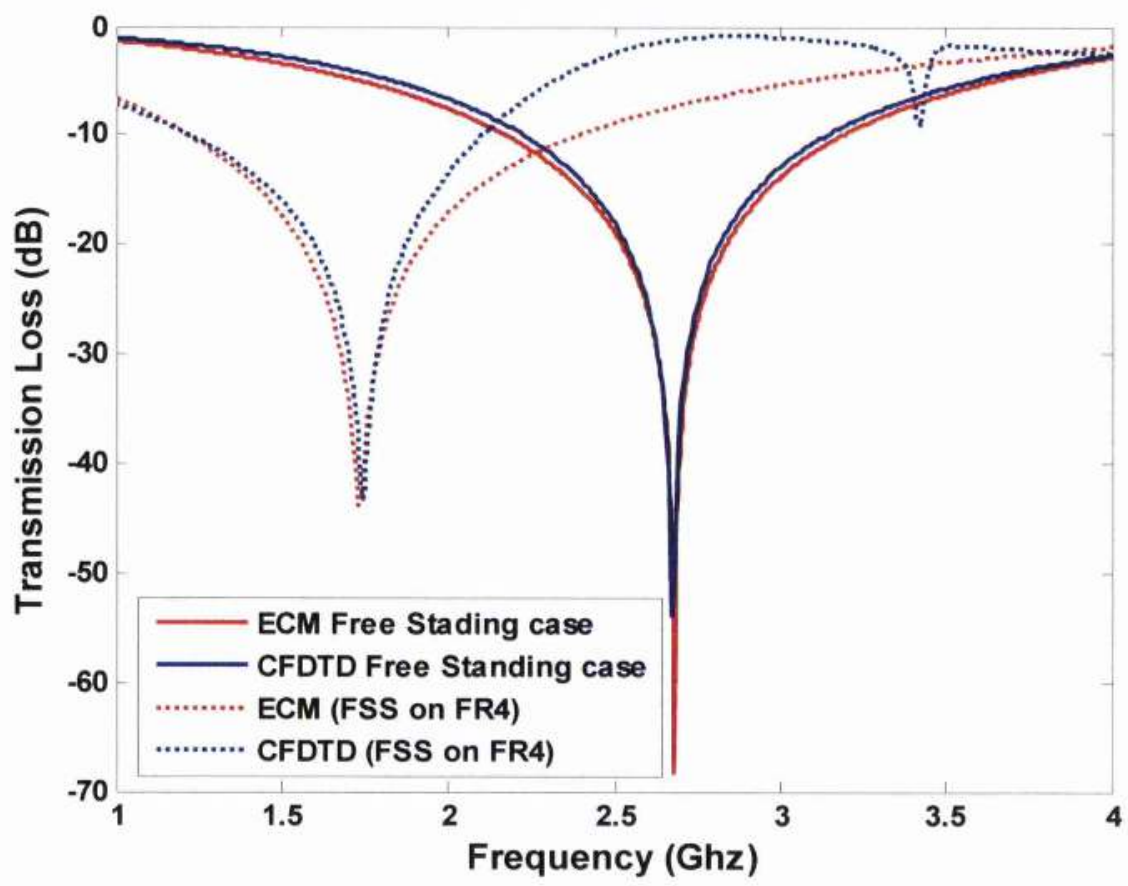

Figure 5-10: Comparison between the Equivalent Circuit method (ECM) and the CFDTD method for a square loop FSS at normal incidence

Figure 5-10 demonstrates a comparison between ECM and the CFDTD method under normal angle of incidence. Both the free standing case (FSS are assumed to be on the air) and the case where the square loop FSS is supported with FR4 material $\left(\varepsilon_{\mathrm{r}}=4.55, \tan \delta=0.0175\right)$ which has thickness greater than $\lambda / 4$ are presented. As already mentioned in Section 5.1.3 when the supporting dielectric has thickness greater than $\lambda / 4$ the FSS tuning frequency converges to to a value equal to $\frac{f_{o}}{\sqrt{\left(\varepsilon_{r}+1\right) / 2}}$ where $f_{o}$ is the resonant frequency of the free standing case. This is verified once more in Figure 5-10. For the free standing case the square loop FSS tunes at $2.7 \mathrm{GHz}$ where as for the case where the FSS is backed with FR4 material it tunes at $1.65 \mathrm{GHz}$.

\subsection{Frequency Selective Surfaces on Building Materials}

\subsubsection{Free-Standing Case}

For the purpose of this investigation a square-loop FSS was designed and fabricated on FR4 dielectric $\left(\varepsilon_{\mathrm{r}}=4.55, \tan \delta=0.0175\right)$. In this work the square loop design was selected mainly because of its superiority with regards to its angular insensitivity over the other element shapes [70] (see Table 5-1). The simulations were performed using the CFDTD method. For simulation 
purposes, the FR4 effective dielectric constant was set to 2.775 as suggested by $\varepsilon_{\text {eff }}=\left(1+\varepsilon_{\mathrm{T}}\right) / 2$ [83][96].

\subsubsection{Experiment Setup}

To characterize the FSS response, measurements were carried out in the anechoic chamber of the Centre for Communication Systems Research in the University of Surrey. DoubleRidged Waveguide Horn antennas (see Appendix 1) have been used for transmission and reception and a Vector Network Analyzer (Rohde and Schwarz VNA ZVCE 20KHz-8GHz) was used to sweep the frequency between 1-4 GHz. The measurement setup is shown in Figure 5-11. It consists of a wooden board, covered with aluminium foil and absorbing material, which has a $72 \mathrm{~cm} \times 72 \mathrm{~cm}$ hole in the middle for FSS placement.

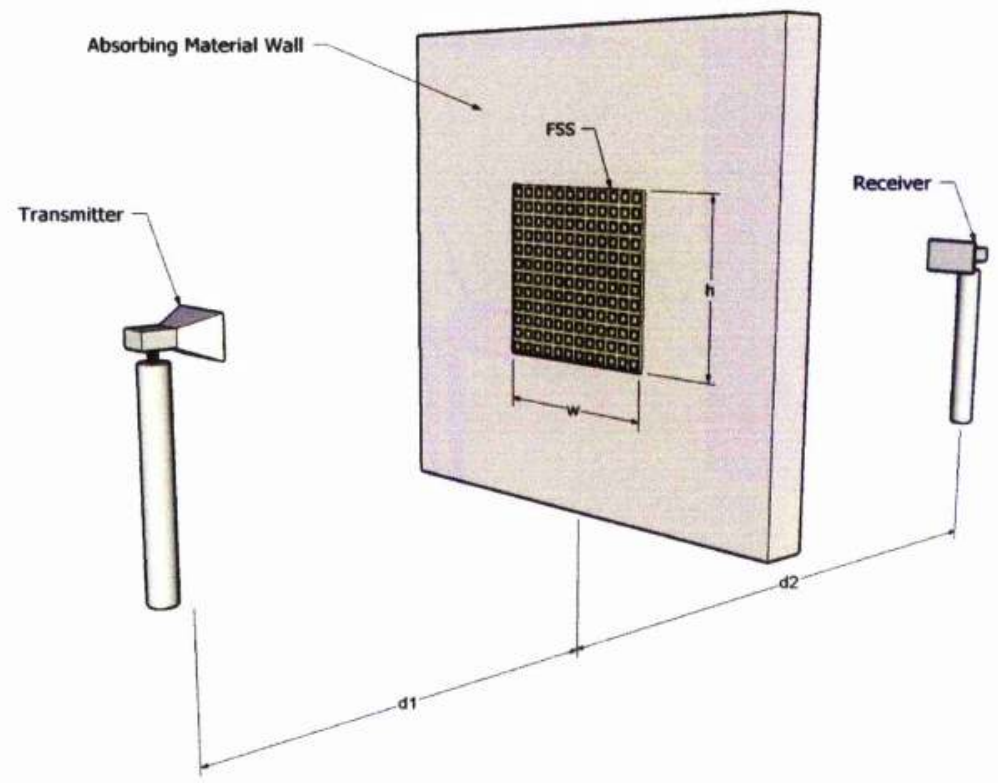

Figure 5-11: Anechoic Chamber setup for FSS transmission loss measurements

It was ensured that the FSS lies in the far field region of the two antennas. The minimum antenna far field distance is given by,

$$
d_{\text {min,FarField }}=2 D^{2} / \lambda
$$

where $D$ is the diameter of the antenna or of the smallest sphere which completely encloses the antenna. The horn antennas used have $D=0.22 \mathrm{~m}$, hence the minimum distance that these antennas can be placed away from the FSS structure, for a frequency range $1-4 \mathrm{GHz}$ is $d_{\text {min,FarField }}=$ $d_{1}=d_{2}=1.3 \mathrm{~m}$. In order to ensure that all the contributions arriving at the receiving antenna are all in phase, the $60 \%$ of the $1^{\text {st }}$ Fresnel Zone should be kept unobstructed [4]. This is done by 
ensuring that : $h / 2 \geq r_{0.6}$ and $w / 2 \geq r_{0.6}$ where $r_{n}$ is the radius of the $n$th Fresnel Zone as shown in Figure 5-12 and given by [4]:

$$
r_{n}=\sqrt{\frac{n \lambda d_{1} d_{2}}{d_{1}+d_{2}}}
$$

Where $r_{n}$ is the radius of the $n$th Fresnel Zone as shown in Figure 5-12.

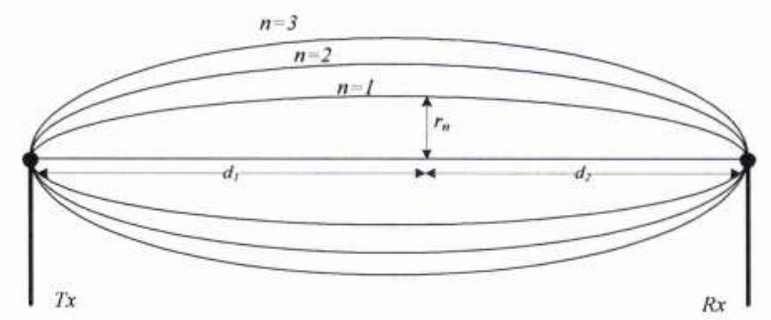

Figure 5-12: Fresnel Zones

For this setup $r_{0.6}=0.34 \mathrm{~m}$ which satisfies the aforementioned conditions.

It has to be noted that all the results presented in this chapter have been time-gated with a $5 \mathrm{~ns}$ timing window to eliminate any possible unwanted contributions (e.g. diffractions around the edges of the wooden board).

Figure 5-13 presents the simulated and measured transmission characteristics of the square-loop FSS under normal angle of incidence. The measurement result is in close agreement with the simulated result which verifies the validity of the CFDTD method used.

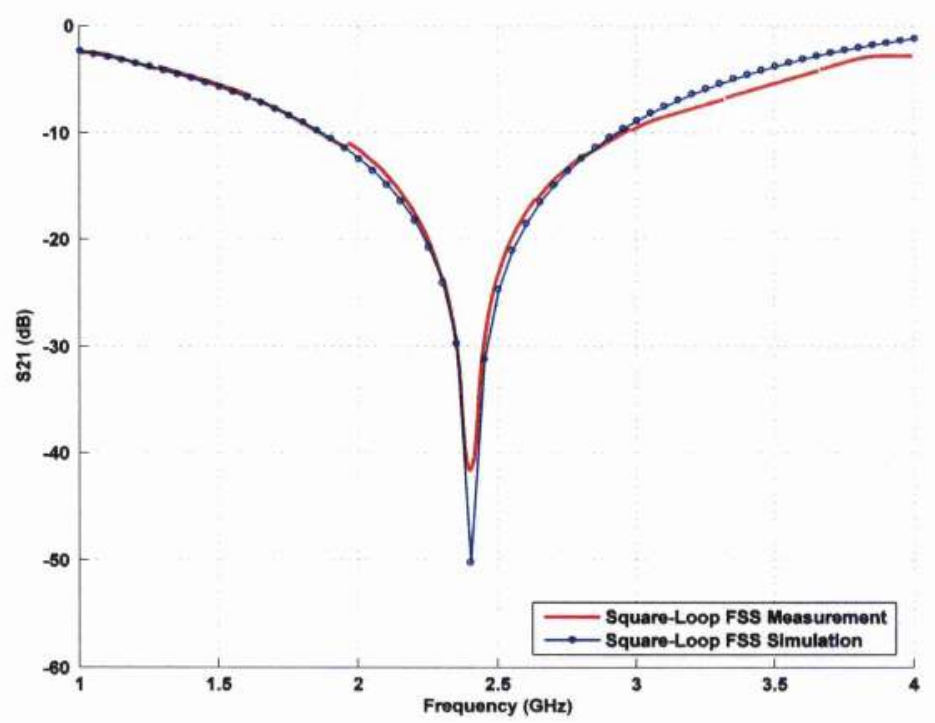

Figure 5-13: Transmission through a square-loop FSS under normal angle of incidence 
Figure 5-14 presents CFDTD simulation results of the transmission (S21) and reflection (S11) characteristics of the square-loop FSS, under four angles of incidence $\left(0^{\circ}, 18^{\circ}, 36^{\circ}\right.$ and $\left.54^{\circ}\right)$, for TE and TM polarisations respectively. It is clear that as the angle of incidence is varied, the transmission and reflection characteristics will vary as well. Thus, when designing a FSS for a particular scenario, this angular sensitivity has to be taken into account in order to correctly model the appropriated radio wave interaction in the modelled environment.
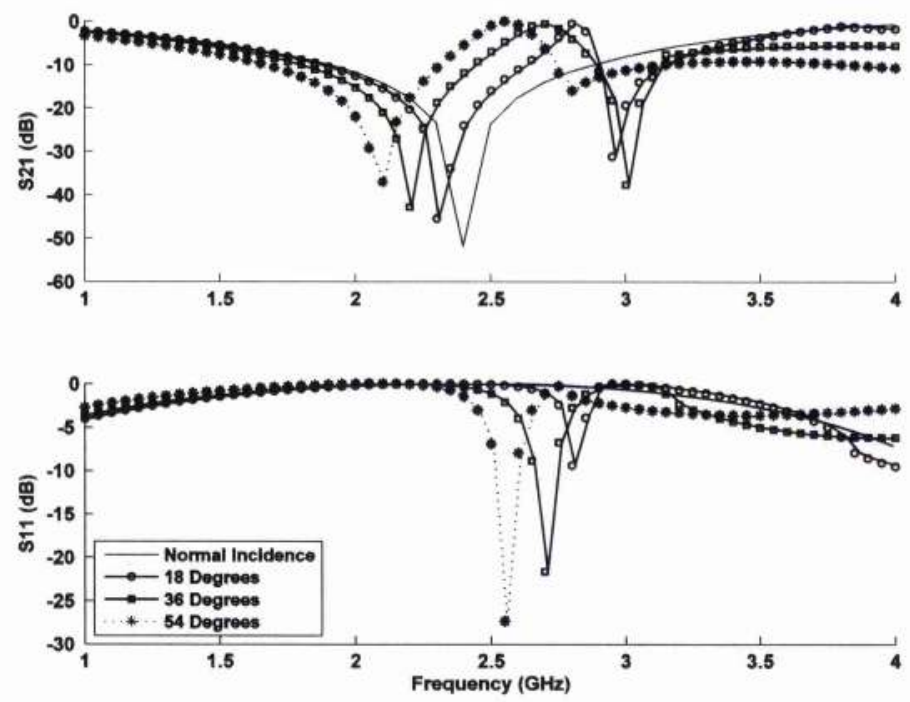

(a)
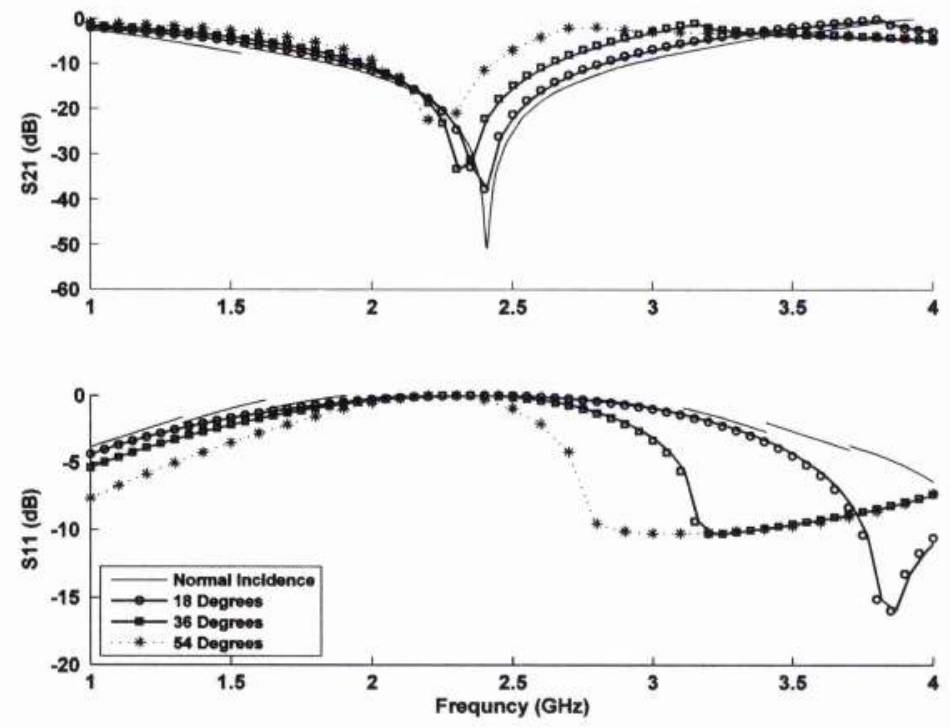

(b)

Figure 5-14: Transmission (S21) and Reflection (S11) loss of the square loop FSS for various angles of incidence. (a) TE polarisation (b) TM polarisation 


\subsubsection{Deployment on Building materials}

So far the discussion was concerned with the FSS free standing case. For the application suggested, the FSS need to be deployed on building materials. Literature reports that when a FSS is placed on a dielectric medium, its standalone frequency response will change [65], [96]. Therefore, the interaction of building walls and FSS has to be investigated when considering this kind of application. The first step into this investigation was to measure the constitutive parameters of typical homogeneous building materials used for this study.

The real and imaginary parts of the complex dielectric constant $\varepsilon_{r}=\varepsilon^{\prime}-j \varepsilon^{\prime \prime}$ have been calculated through the use of a multiple pass technique [97] where, an insertion transfer function $H(j \omega)$ that accounts for multiple reflections inside the dielectric slab is required. This function is defined as the ratio of the frequency domain signals in the presence $V_{\text {mat }}(j \omega)$ and absence $V_{\text {free }}(j \omega)$ of the materials under test.

$$
H(j \omega)=\frac{V_{\text {mat }}(j \omega)}{V_{\text {free }}(j \omega)}
$$

The insertion transfer function is linked to the transmission coefficient $S 21$ as,

$$
S_{21} e^{j \beta_{o} d}=H(j \omega)
$$

where $\beta_{o}=2 \pi f / c$ and $d$ is the thickness of the dielectric slab. The S21 parameter which can be directly measured in the anechoic chamber using the setup shown in Figure 5-11 can be cast into the following equation:

$$
\left(x+\frac{1}{x}\right) \sinh (x P)+2 \cosh (x P)-\frac{2}{S_{21}(j \omega)}=0
$$

where $x=\sqrt{\varepsilon_{r}}, S_{21}(j \omega)=H(j \omega) e^{-j \omega \tau_{o}}, \tau_{o}=c / d$ and $P=j \beta_{o} d$. This equation is solved numerically for the complex dielectric constant $\varepsilon_{r}=\varepsilon^{\prime}-j \varepsilon^{\prime \prime}$ using two dimensional search algorithms.

For the purpose of this research two materials have been characterised in terms of their electrical properties; a 9mm plaster board and a 9mm MDF (wooden) board. Figure 5-15 and Figure 5-16 show the obtained relative permittivity and loss tangent respectively for these two materials as a function of frequency.

To obtain an initial indication of the effect on the FSS response when the latter are deployed on building materials, the square loop FSS presented in section 5.3.1 was attached on these two materials. 


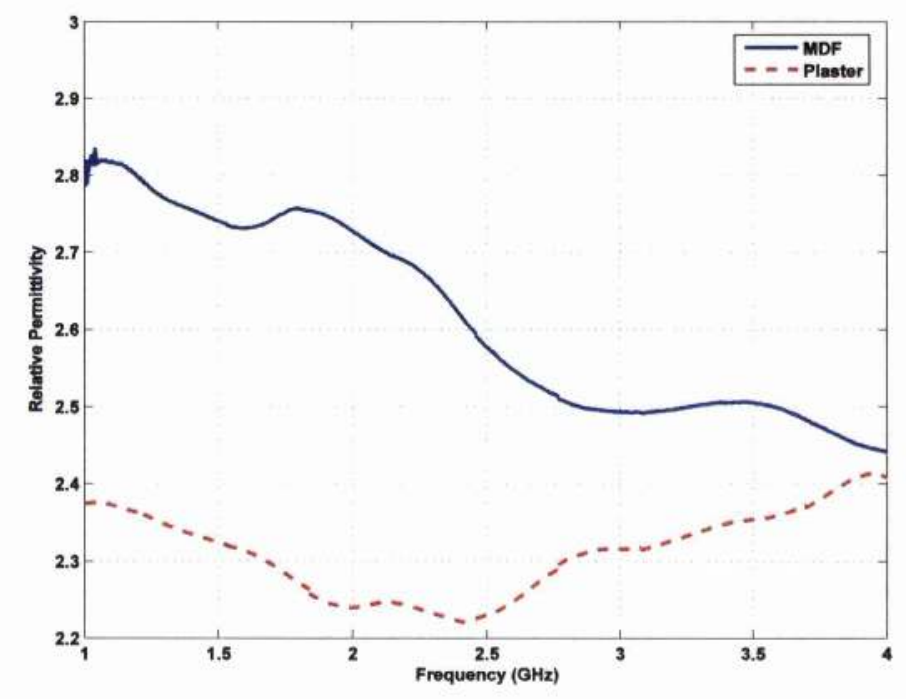

Figure 5-15: Relative permittivity of plaster and MDF (wooden) boards

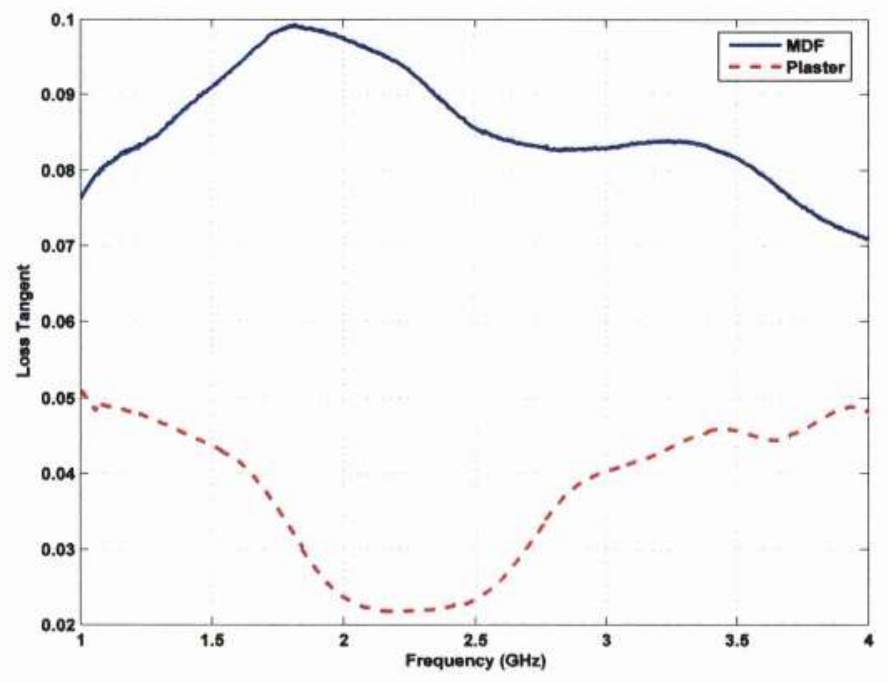

Figure 5-16: Loss Tangent of plaster and MDF (wooden) boards

Figure 5-17 presents through both CFDTD simulations and measurements the effect of a 9mmthick plaster board and a 9mm-thick MDF board on the frequency response of the square loop FSS. The results indicate a decrease on the tuning frequency of the FSS when the latter is attached onto the building material. This is due to the fact that low-order evanescent Floquet modes, which decay exponentially with distance away from the elements, still have significant amplitudes at the boundary, hence modifying their relative amplitudes and the energy stored close to the array (FSS) and thus affecting the resonant frequency. Similar behaviour has been observed in [65] where the effect thickness of the supporting dielectric layer has been investigated (see section 5.1.3) 


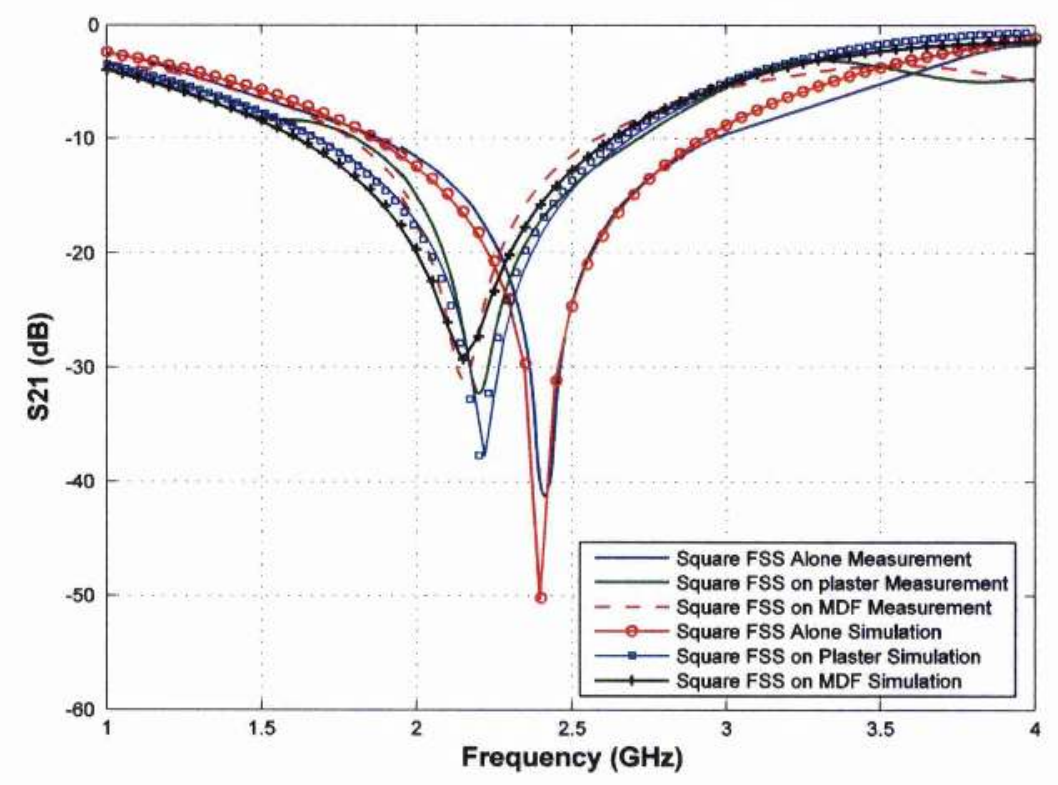

Figure 5-17: Effect of wood and plaster when these are attached on the square loop FSS

To further investigate this effect, simulations and measurements have been performed by varying the distance between the FSS and the material.

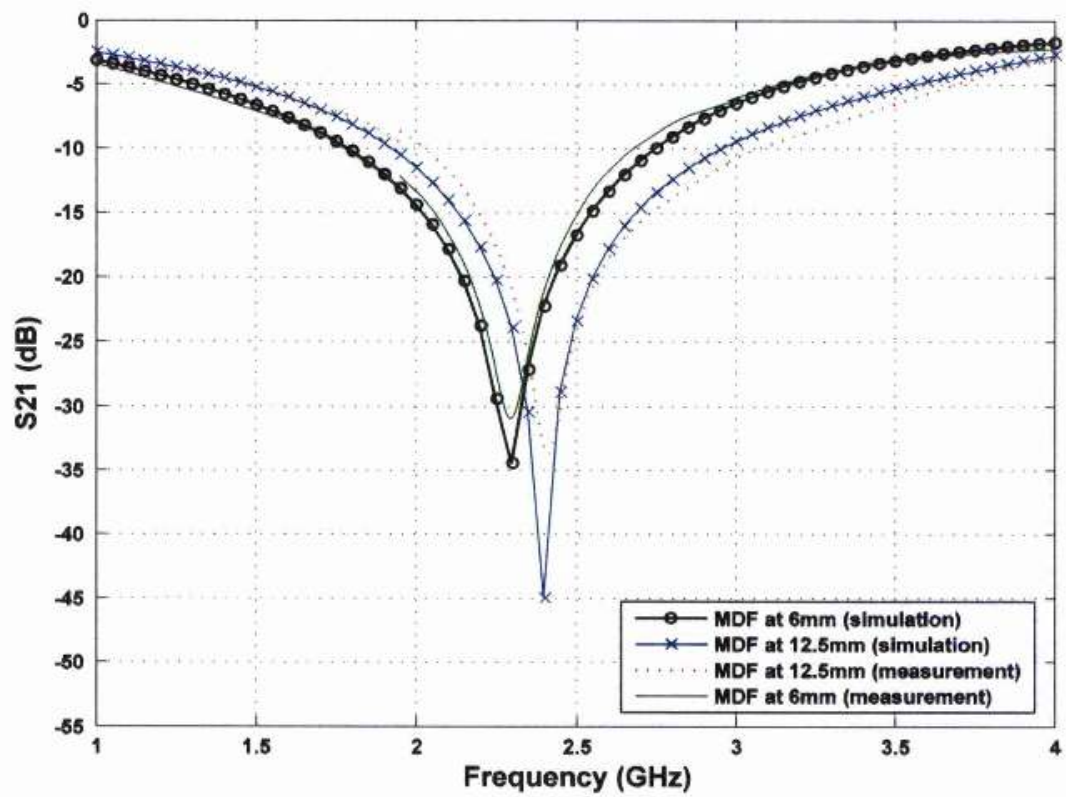

Figure 5-18: Effect of varying the air gap distance between the square loop FSS and a $9 \mathrm{~mm}$ wooden board at normal incidence 
Figure 5-18 presents simulations and measurement results after varying the air gap between the FSS and a wooden building wall. It is observed that by moving the FSS away from the building interface, the frequency response approaches the free standing case. The first finding was that when the FSS was placed at $12.5 \mathrm{~mm}$ away from the wooden board, the tuning frequency of the FSS has reached its free-standing value. To further study this effect, simulations and measurements have been carried out so as to obtain a "break-point" air gap, beyond which the FSS frequency response is not affected.

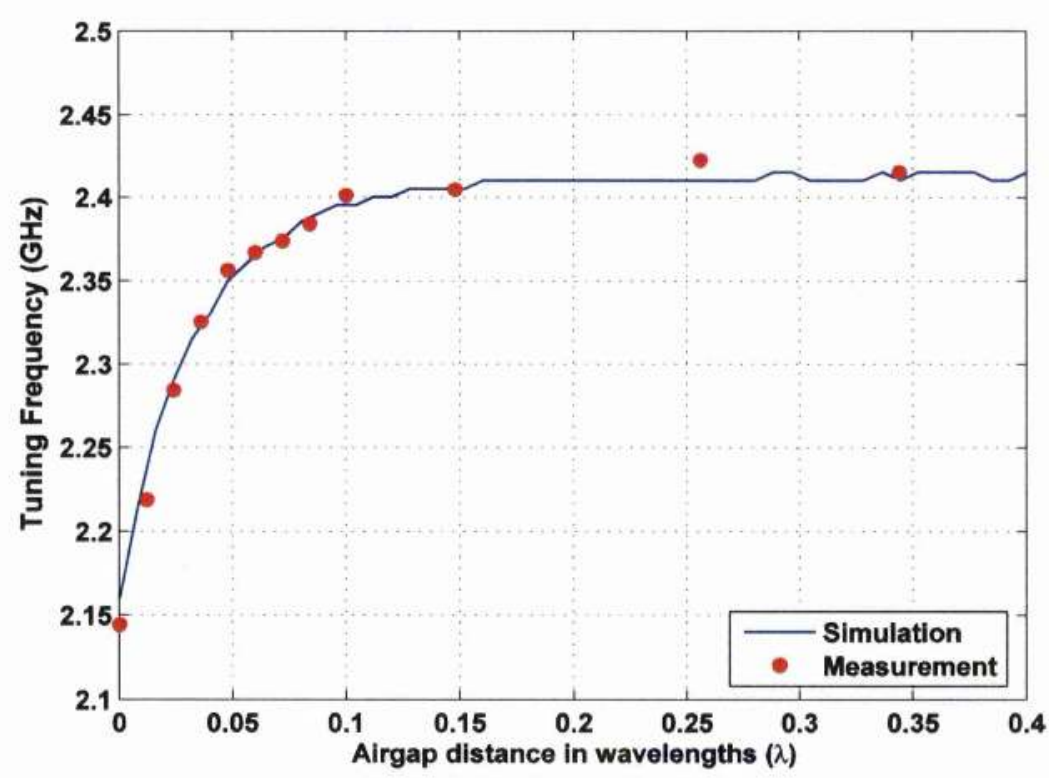

Figure 5-19: Simulation and measurement of the effect of the air gap between the square-loop FSS and a $9 \mathrm{~mm}$-thick wooden (MDF) board on the tuning frequency.

Figure 5-19 indicates that the FSS tuning frequency increases rapidly as the air-gap increases from 0 to $\lambda / 20$ and converges to the free-standing value for air-gaps beyond $\lambda / 10$, eliminating any detuning effects. Further investigation has been carried out through CFDTD simulations to study this air-gap relevance to the frequency, the building material electrical parameters and thickness as well as the FSS design [94].

Figures 5-20 to 5-22 present CFDTD simulation results obtained by changing the thickness and electrical properties of the building wall, the FSS element shape and FSS standalone frequency. These results suggest that these parameters do not have any effect on the "safe-breakpoint" (N10) distance that the FSS can be deployed away from the building wall, so as its freestanding FSS response is not affected. 


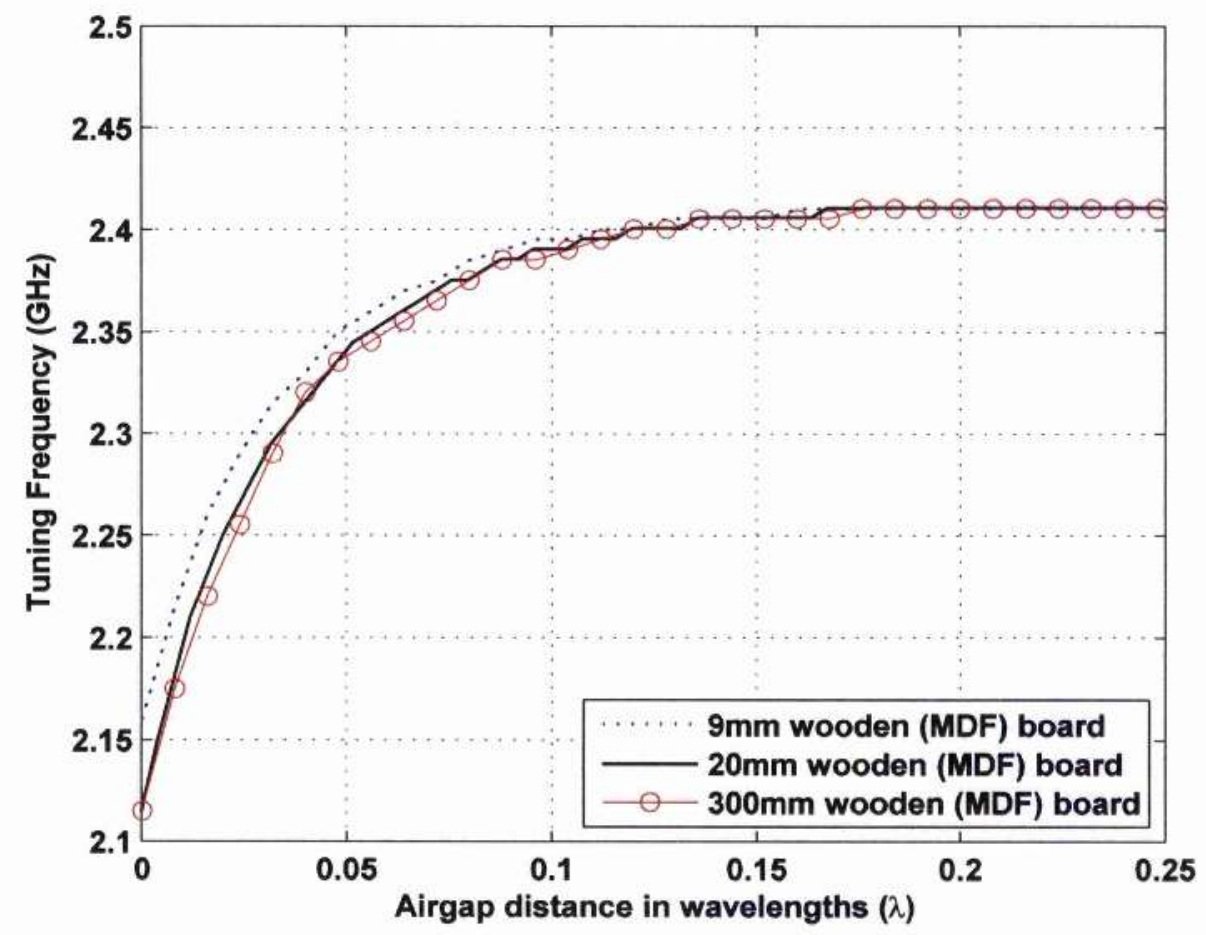

Figure 5-20: Air gap effect for various MDF thicknesses on the square loop FSS response

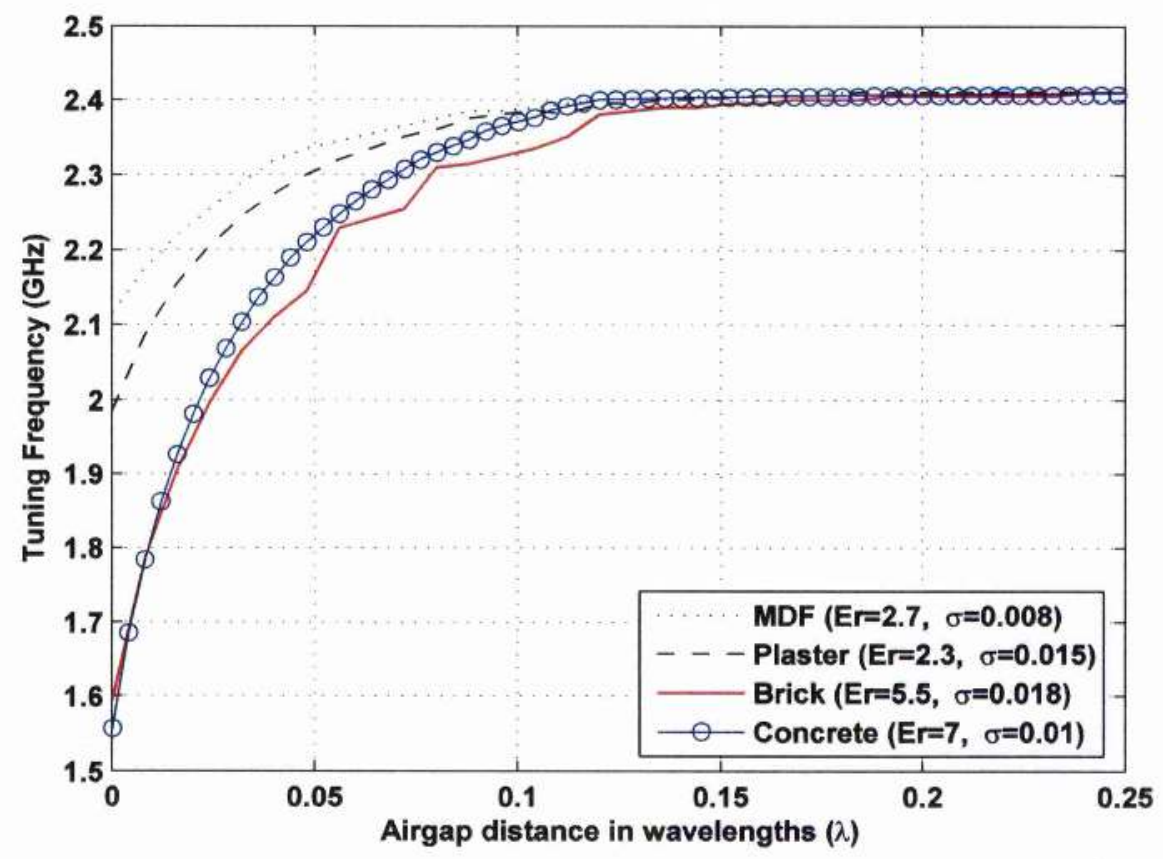

Figure 5-21: Air gap effect for various $20 \mathrm{~mm}$-thick building materials 


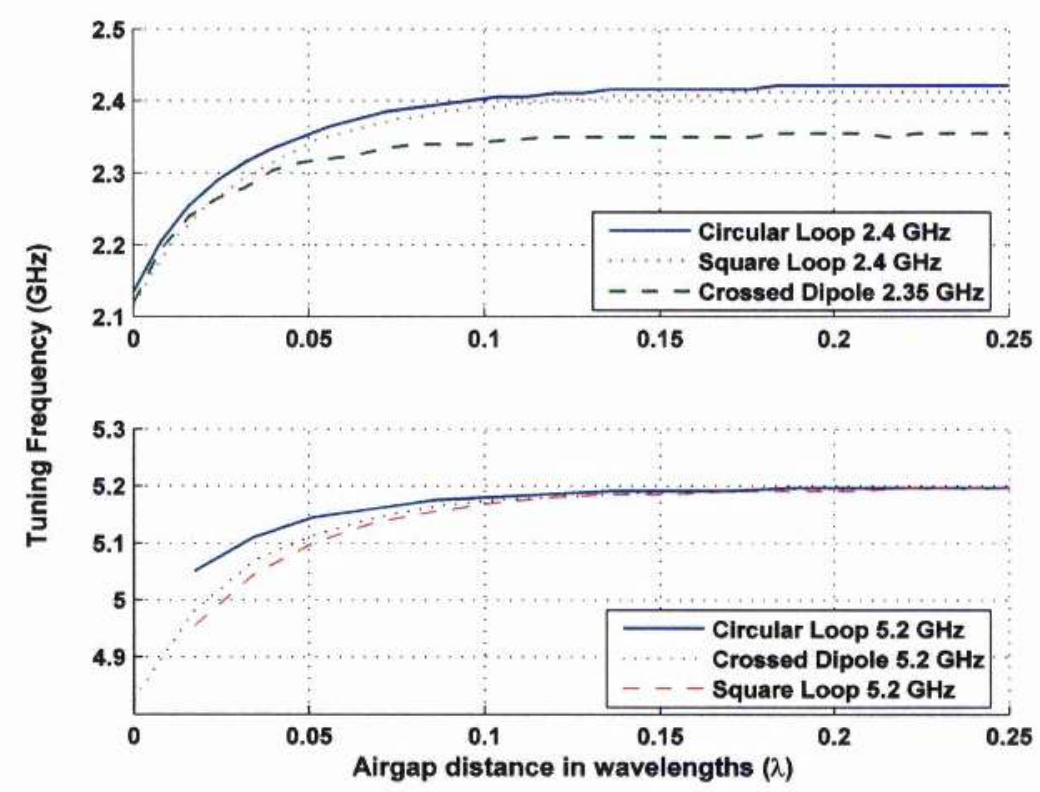

Figure 5-22: Air gap effect for various FSS designs at 2.4 and $5.2 \mathrm{GHz}$ deployed on 20mm-thick MDF

\subsubsection{Deployment on periodic structures}

The investigation so far was concerned with the deployment of FSS on homogeneous building materials. It would be interesting to study whether the safe break-point distance examined earlier for homogeneous structures applies for building walls which their internal structure exhibits a certain periodic pattern. For this reason, CST Microwave Studio simulations have been carried out for the square loop FSS used in the previous investigation, deployed on a reinforced concrete wall, the geometrical parameters of which are shown in Figure 5-23. The concrete permittivity was set to $\varepsilon=7-j 0.3$.

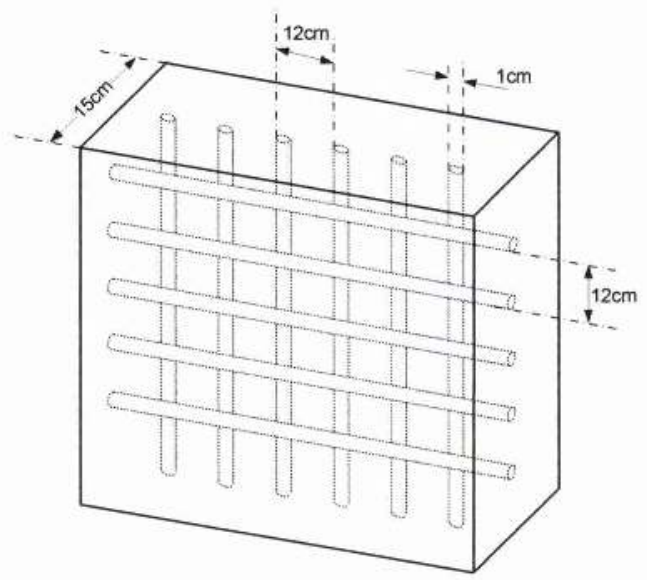

Figure 5-23: Reinforced concrete geometry for FSS deployment 


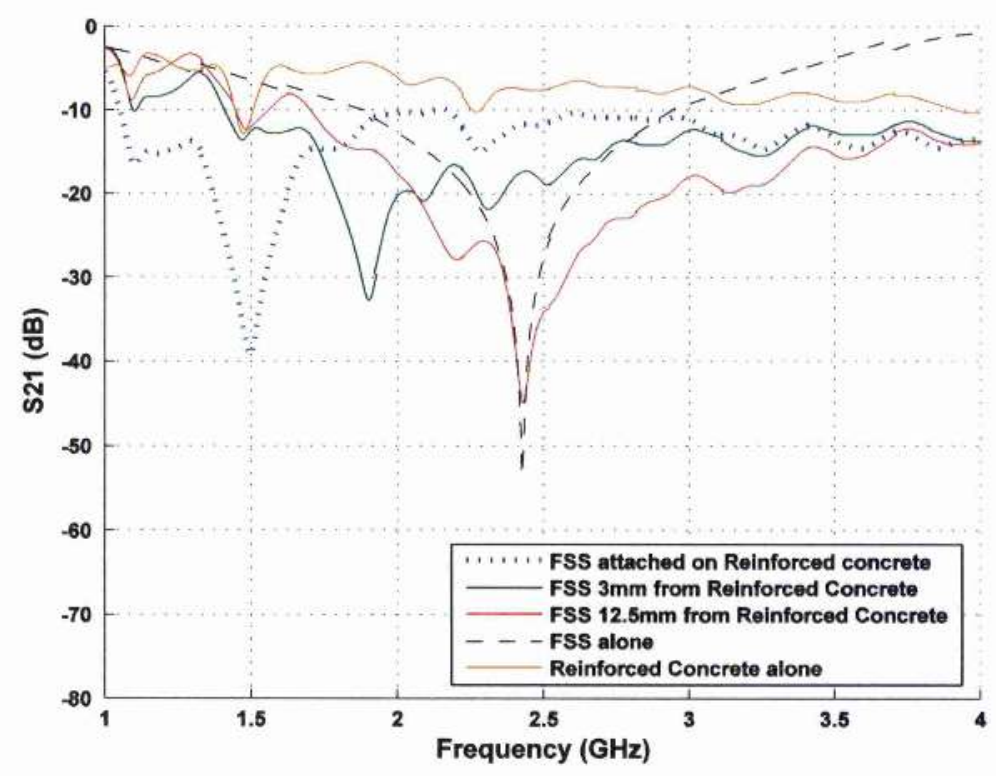

Figure 5-24: Effect of a reinforced concrete wall on the square-loop FSS frequency response

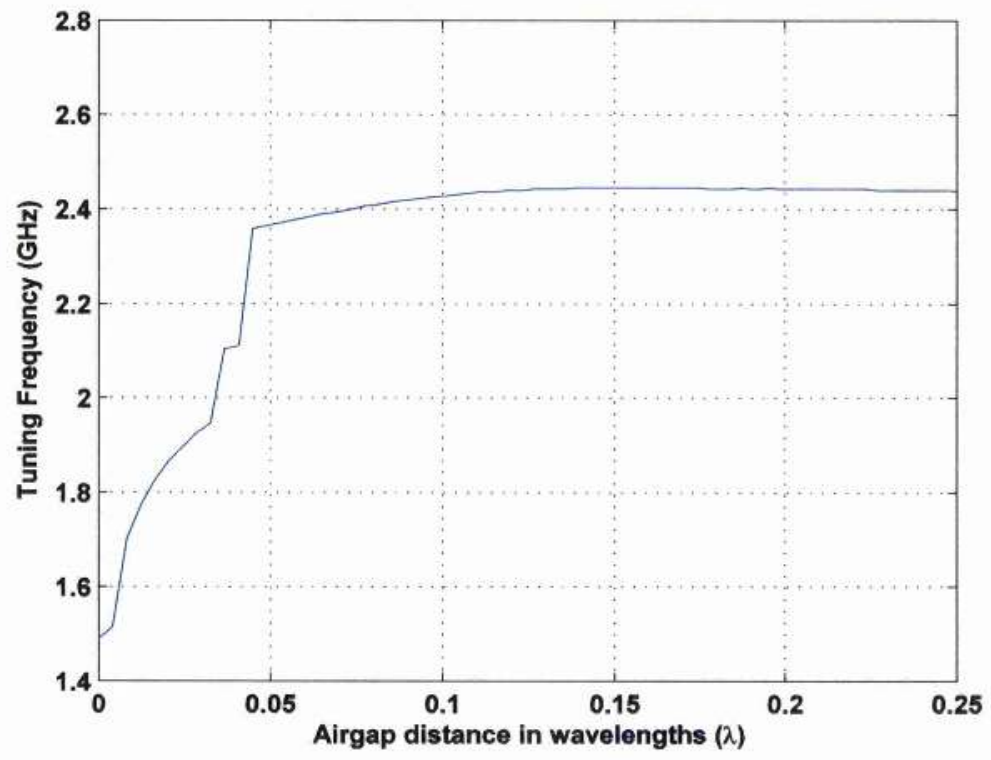

Figure 5-25: Effect of the air gap between a reinforced concrete wall and a square loop FSS on the tuning frequency

Figure 5-24 and Figure 5-25 demonstrate that like in the homogeneous building materials case, a periodic structure has similar effect on the frequency response of the FSS. The same safe "break point" air gap $(\lambda / 10)$ obtained for the homogeneous structures applies for periodic ones as well. 


\subsection{Summary}

The basic principles governing the operation and analysis of FSS have been presented in this chapter. This has included a brief overview of the FSS basic characteristics, the various types of element shapes, the role and effect of the supporting dielectric substrate, some typical applications of FSS reported in literature, as well as a brief talk about the most commonly used FSS analysis techniques. Practical considerations regarding the deployment of FSS in wireless environments, such as the angle of incidence, and polarisation of the incident wave have been demonstrated.

Extensive investigation has been carried out through both CFDTD simulations and anechoic chamber measurements to study the effect of deploying frequency selective surfaces on conventional building materials. It was observed that once the FSS are attached on building walls detuning effects take place, which depend on the electrical parameters and dimensions of the supporting walls. For the proposed FSS application in wireless environments, it would be currently difficult, if not impossible, to precisely know the wall characteristics. In addition, it is usually the case in indoor and outdoor environments that walls have different morphological and geometrical properties. This would necessitate fabrication of various FSS designs so as the overall structure (FSS-wall) be tuneable to the desired frequency considering also the different effects of the different supporting walls. Therefore the investigation was concentrated on finding a "breakpoint" air-gap, beyond which the FSS response is not affected by the presence of any building wall. It was observed that for air-gaps greater than $\lambda / 10$, the FSS standalone tuning frequency is not affected by any homogeneous or periodic structure that exists behind it [94]. This air-gap appears to be dependent only on the wavelength of transmission and is not affected by the FSS design and the morphological/geometrical properties of the building walls. This would effectively give the flexibility to the designer to fabricate a single FSS design and safely deploy it on any building wall without worrying about any possible detuning effects.

It is common practice that prior to the implementation of any wireless system, a radio prediction tool is used to predict radio coverage in order to optimise the design. For the proposed application such a tool needs to incorporate the FSS behaviour as well. The next chapter presents a deterministic 3D Ray Tracing model which incorporates this FSS behaviour. 


\section{Chapter 6}

\section{Modified Ray Tracing Model to account for FSS behaviour}

To study the effect of FSS when these are deployed in wireless communication environments, a Ray Tracing model has been developed in MATLAB and modified to incorporate the FSS frequency characteristics. As previously discussed, Ray-Tracing algorithms are classified either as direct (ray-Launching) or inverse (Image method). Image method imposes high computation due to the increased number of shadowing tests required. However, since using the image method, the entire computation domain of receivers can be handled on the same time using a matrix and since MATLAB offers the ability and flexibility to easily manipulate large matrices, this method has been used for the implementation of the propagation model. To improve performance, some image-method-specific acceleration techniques have been utilised. Since, in this model, the geometry of each ray is examined in 3D space, both the azimuth and elevation angles of arrival at the receiver or any other interaction point are available, and hence, the model is also capable of using 3D antenna radiation pattern. The FSS behaviour has been incorporated into the model as a form of angle of incidence dependent reflection and transmission coefficients obtained using CFDTD analysis.

This chapter presents the implemented Ray-Tracing algorithm and compares its results with and measurements carried out in an indoor office environment. It also verifies the applicability of the modified FSS-Ray Tracing model through measurements carried out in a small-scale indoor environment constructed in an anechoic chamber [98].

\subsection{Environment Modelling}

The first step, during the implementation of the algorithm was to develop an unambiguous way for defining the environment. For this reason an environment database was developed which allows the definition of the transmitter location and the geometrical and morphological description of the environment facets. The MATLAB structure was utilised for this purpose, which allows the organisation of the data in a hierarchical manner for easier manipulation. The 
variable Building was defined as the root of the structure, which contains all the other information as illustrated in the figure below:

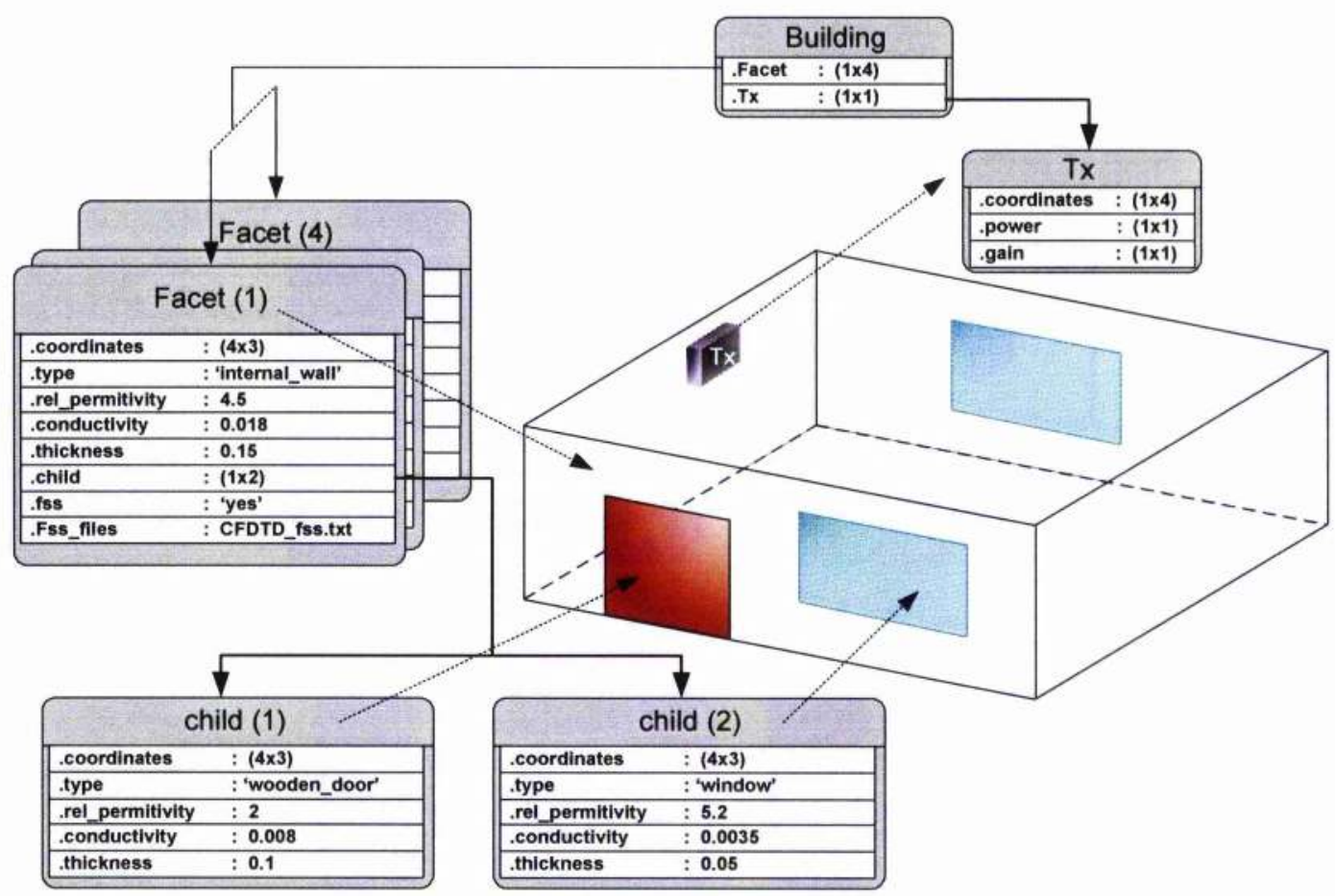

Figure 6-1: Ray Tracing Algorithm Environment Description

The structure Building is thought to logically contain two other structures; the first one is the transmitter (structure $\mathrm{Tx}$ ) and the second one is the Facet structure. The $\mathrm{Tx}$ structure contains three properties; the 3D Cartesian coordinates (.coordinates) of the transmitter's location, its transmitting power (.power) and its gain (.gain). The Facet structure contains all the defined facets inside the environment. Each Facet is a structure itself containing eight properties; the 3D Cartesian coordinates of the facet's vertices (.coordinates), the facet's type (.type), relative permittivity (.rel_permitivity), conductivity (.conductivity) and thickness (.thickness), a flag that indicates whether an FSS is attached to it, a string that contains the file name of the CFDTD reflection and transmission coefficients of the deployed FSS. The .child property provides a link to other facets that are contained within the current facet (e.g. doors or windows). By using this child relationship we avoid the case of defining new facets for which the image to be calculated will be the same as the parent image. This saves computation load and time.

Based on this scene modelling, the environmental description of a given indoor scenario can be saved into a separate file and loaded upon execution. 


\subsection{The image tree algorithm}

Once the environmental description of a given indoor scenario is loaded the first step of the algorithm is to construct an image tree, with as many levels of images as needed to compute the field strength to the desired level of accuracy [99]. Basic computer graphic techniques based on 3D transformations such as translation, rotation and reflection matrices have been utilised to compute the image point of a source with respect to an arbitrary placed and arbitrary shaped facet [100]. In order to discard the images that do not exist in practice, shadowing tests are performed during the calculation of the image tree. According to Figure 3-12(b) when a facet is completely outside the reflection region of a source (or image) point, then the image that corresponds to that facet can be discarded. Implementing this condition has showed that the number of images was reduced by approximately $25-40 \%$ according to the geometry of the modelled environment, considerably reducing the computation overhead and time to produce results. The image tree is calculated and stored in a two dimensional array images $(a, b)$ where a represents the order of each image and $\mathbf{b}$ is a counter for every image. Every element of the array is a structure with a number of properties as shown in the example in Figure 6-2 which shows a $3^{\text {rd }}$ order image:

\begin{tabular}{|lc|}
\hline \multicolumn{2}{|c|}{ images(3,17) } \\
\hline .coordinates & $:(4 \times 3)$ \\
\hline .ref_facet & $: 3$ \\
\hline .parent_source_index 1 & $: 2$ \\
\hline .parent_source_index 2 & $: 3$ \\
\hline .exists & $: 1$ \\
\hline
\end{tabular}

Figure 6-2: Image structure example

The following properties are defined:

- coordinates which identifies the 3D Cartesian coordinates of the image

- . exists states whether the image actually exists or not ( $1=$ exists, $0=$ does not exist). 、

- .ref_facet specifies the facet that the image was calculated from.

- parent_source_index1 and parent_source_index2 which identify the indices of the parent image in the image tree.

The algorithm for the calculation of the image tree is shown in Figure 6-3: 


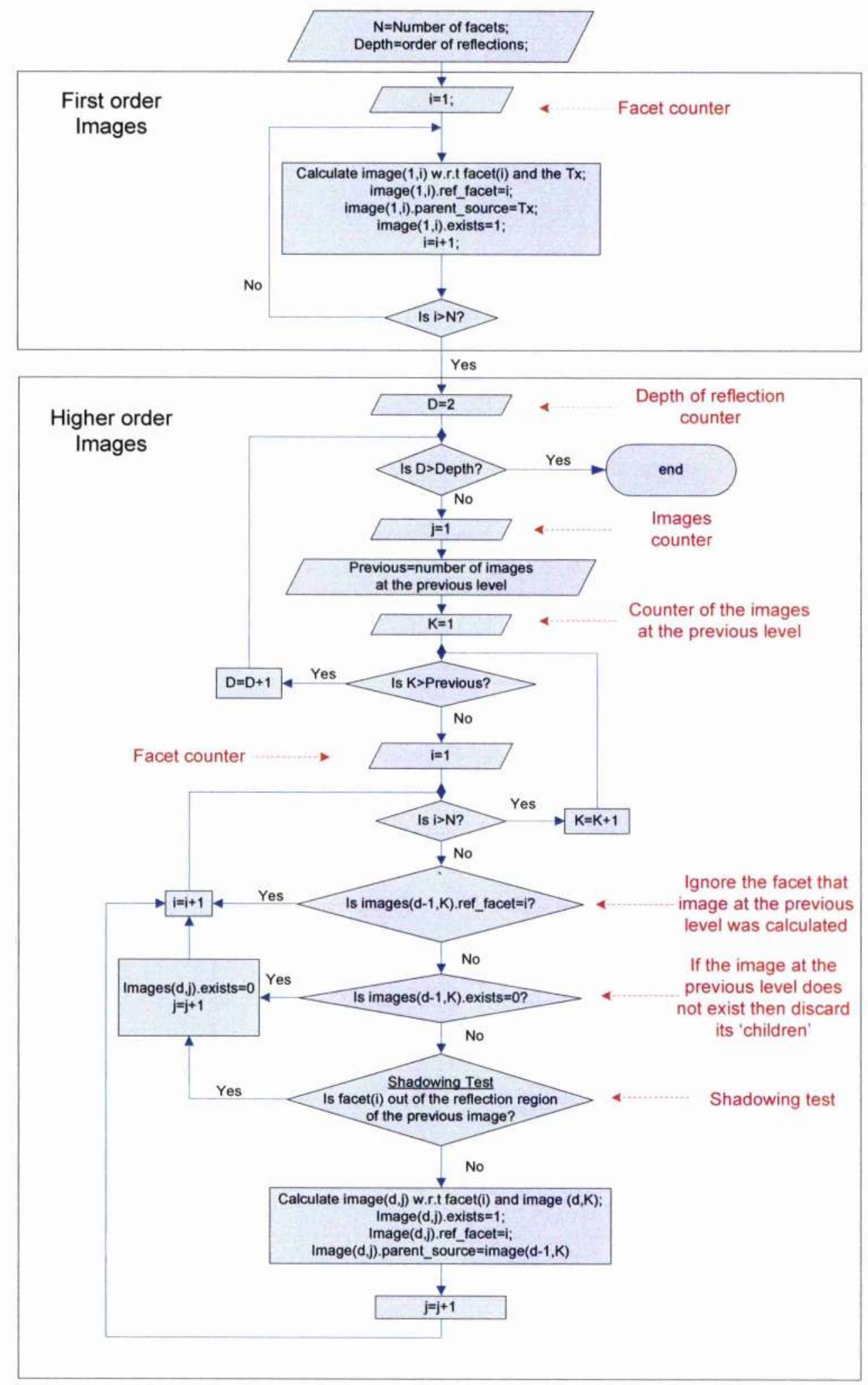

Figure 6-3: The image tree algorithm flowchart 


\subsection{Transmission Algorithm}

The transmission algorithm is a standalone algorithm that calculates only the refraction losses that a ray encounters as if refraction was the only propagation mechanism. For every observation point on the prediction plane (grid), which is defined by three 3D Cartesian coordinate matrices $(x, y$ and $z$ ), a ray is traced back to the transmitter and the transmission coefficients associated with every facet the ray encounters on its way back to the transmitter are calculated using equations (2-45) to (2-51). This results into a matrix that contains the transmission coefficients for every point in the grid as if the facet was the only one present in the scene. Since polarisation and the antenna pattern are taken into consideration three transmission coefficient matrices are calculated which correspond to $x, y$ and $z$ direction respectively. The same procedure is followed for every facet in the environment and all the transmission coefficient matrices are multiplied together so that the contribution of transmission losses through all the facets in the scene for all the grid points is calculated. For Line of Sight $(\mathrm{LoS})$ receiving points the transmission coefficients are equal to unity. For the calculation of the transmission coefficients the angle of incidence is required. This is calculated by finding the dot product between the unit ray vector and the facet unit normal vector.

Once the transmission coefficient matrices are calculated then these are multiplied by the spreading factor and phase delay matrices (obtained by calculating the overall distance between the transmitter and all the possible receiver location in the prediction plane). Finally, the result is multiplied by the transmitted electric field in order to obtain the electric field for every point in the grid taking into consideration transmission losses only. The flowchart of the transmission algorithm is shown in Figure 6-4.

\subsection{Reflection algorithm}

One of the most complex and time consuming part of the propagation model is the algorithm that calculates the electric field due to reflections on the scene's facets and the transmission losses that are encountered when at least one reflection occurs. Once all the realisable images have been determined using the image tree algorithm, a reflection algorithm has been implemented to calculate the reflection coefficients of all possible interactions for the given scenario. Starting from the image at the deepest level of the image tree, the ray is traced back to its parent in the image tree, and then the parent to its parent and so on until the transmitter is reached. If the path exists, then the reflection coefficients associated with all the possible reflections that the ray encounters on its way to the transmitter are calculated. On its way to the transmitter the ray may encounter a number of facets which will attenuate the electric field of the 
observation point under test. Any grid points that are outside the reflection region of any image have reflection coefficients equal to zero.

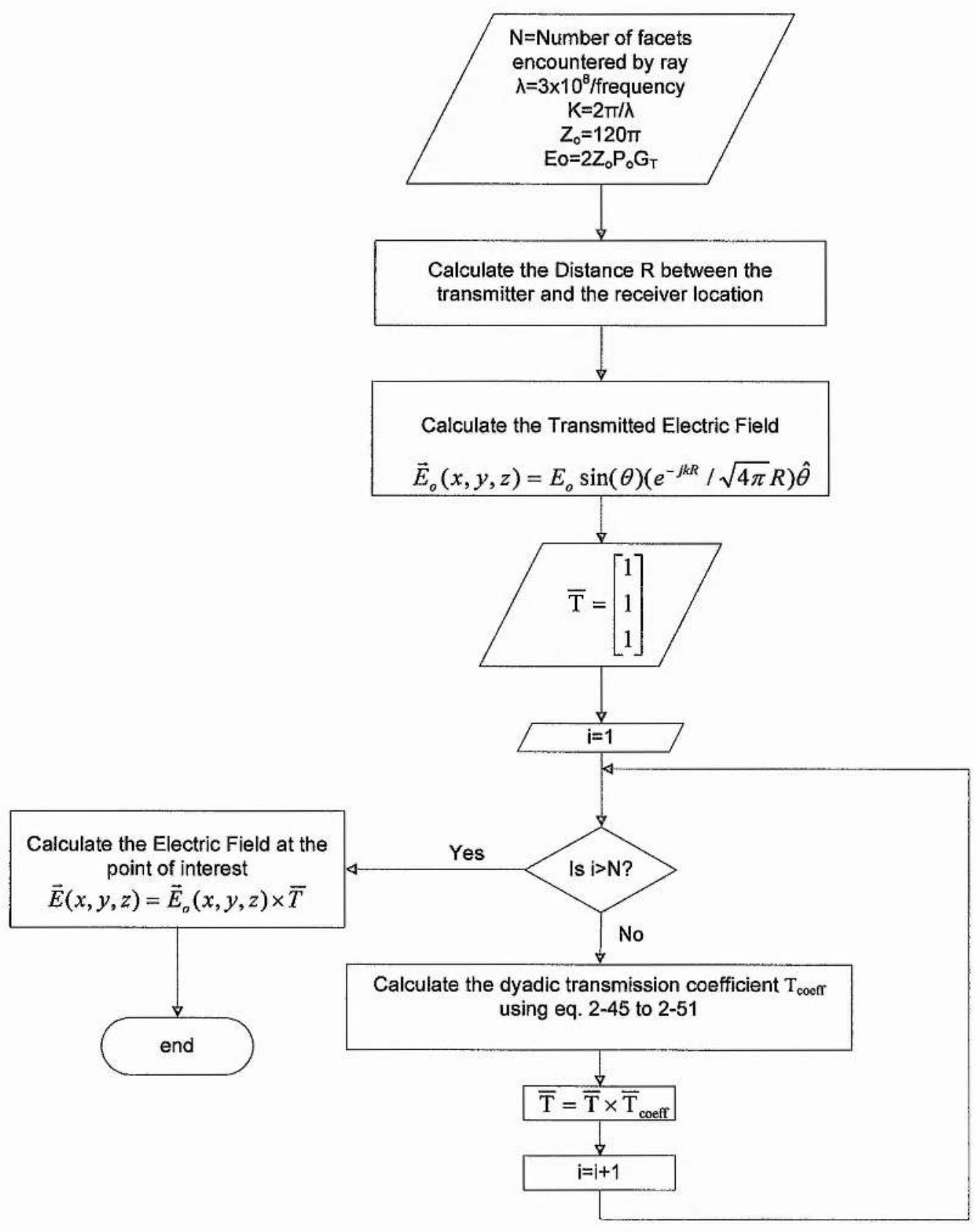

Figure 6-4 : Transmission Algorithm Flowchart

While tracing the reflected ray back to the transmitter, the ray will probably encounter some facets which will attenuate the reflected electric field. The way that the algorithm was built, ensures that all the intermediate transmission coefficients are calculated. However some of these coefficients are equal to unity which means that the corresponding facet is not encountered by the 
traced reflected ray. Therefore in order to avoid the computation of the unnecessary intermediate transmission coefficients, the facets that are not encountered by the traced ray are not considered. In order to do that the shadowing test discussed earlier is used here as well. This discards facets that do not lie between the transmitting antenna and the first reflection facet (Region 1 in Figure 6-5), and facets that do not lie between the receiver location and the last reflection facet (Region 3 in Figure 6-5).

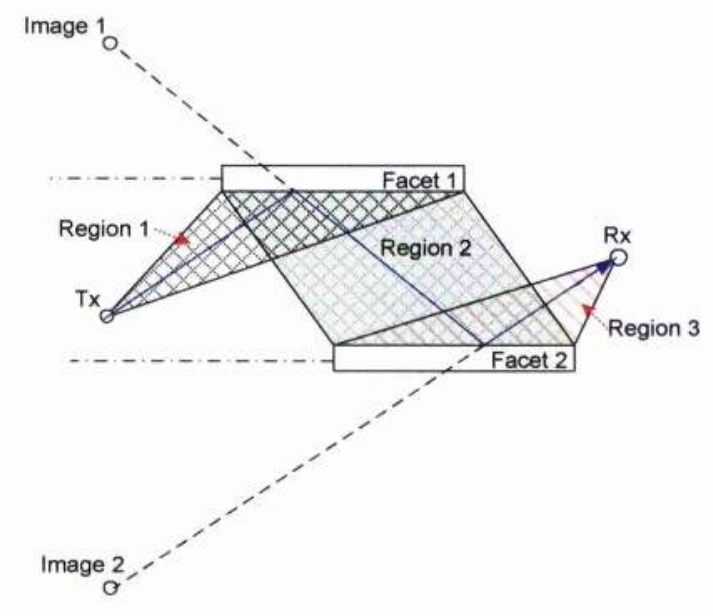

Figure 6-5: Reflection regions

The distance travelled by each ray is also determined in order to allow for the calculation of the path loss and the phase difference from the transmitter to the destination point. As in the case of the transmission coefficients, three reflection coefficient matrices are calculated using equations $2-45$ to $2-51$ in order to account for polarisation and antenna pattern. Once all the Fresnel coefficients for all the points inside the reflection region of the corresponding image are calculated they are multiplied together and the result is multiplied by the corresponding spreading factor (to account for path loss) and the overall phase delay and the resultant coefficients are stored.

The ray-tracing procedure outlined above is repeated for all the images at the deepest level and then the algorithm moves up until all the images in the image tree are taken into account. The coefficients of each image are stored in matrix form (if a ray is not feasible then the coefficient of this observation point is equal to 0 ). All the matrices are finally summed together to obtain the overall coefficients for all the possible observation points in the grid. What is only needed now is to multiply the accumulated coefficients by the transmitted electric field to obtain the electric field of each point in the grid due to reflections which is returned by the algorithm.

The Reflection Algorithm flowchart is presented in Figure 6-6. 


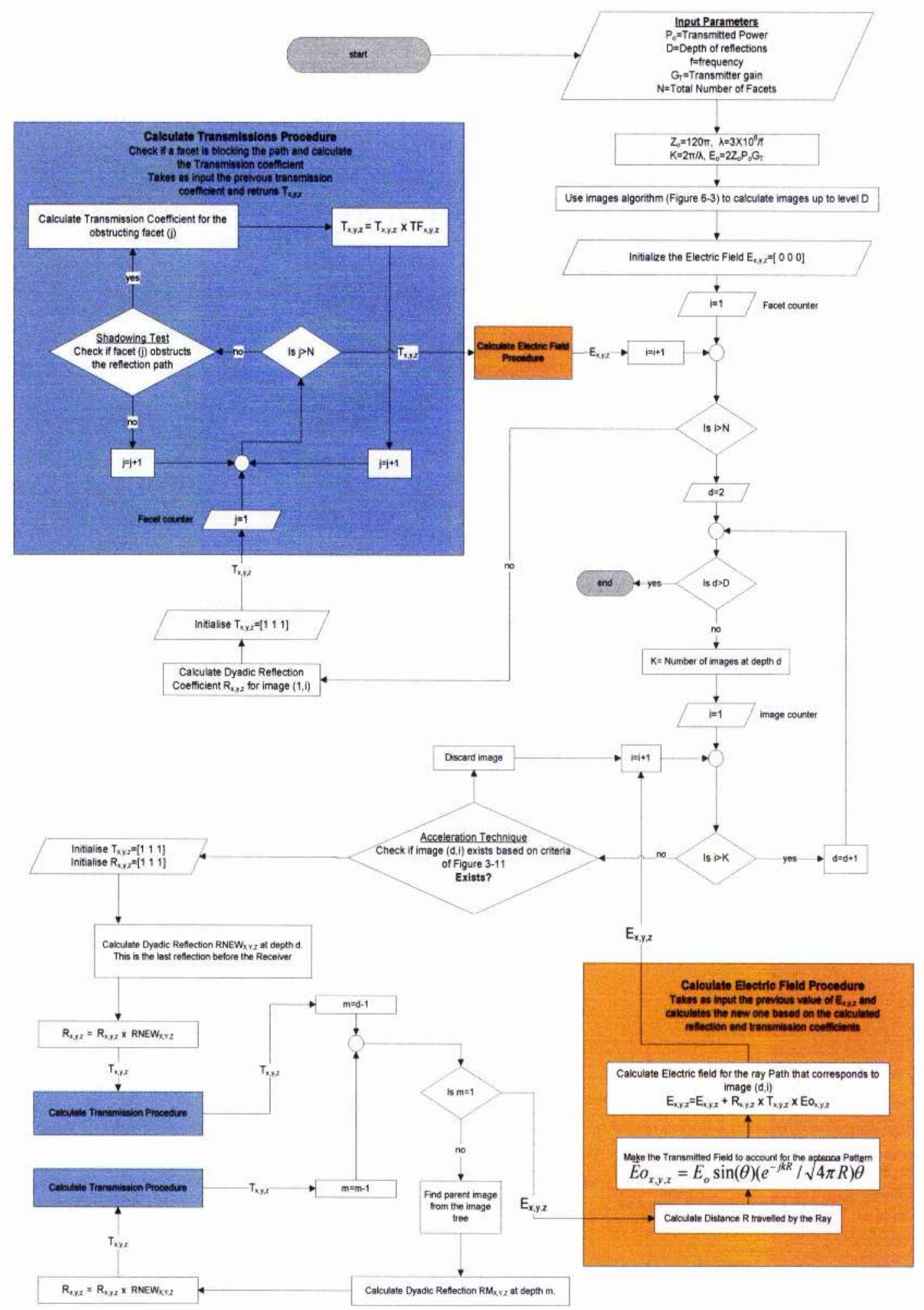

Figure 6-6: Reflection Algorithm flowchart 


\subsection{The Final Model}

The final step in the propagation prediction model is to bind all the previously explained algorithms and procedures so that the power coverage of a transmitting antenna is predicted. The flowchart of the final algorithm is shown in Figure 6-8. It starts with the definition of the various parameters and the loading of the environment description.

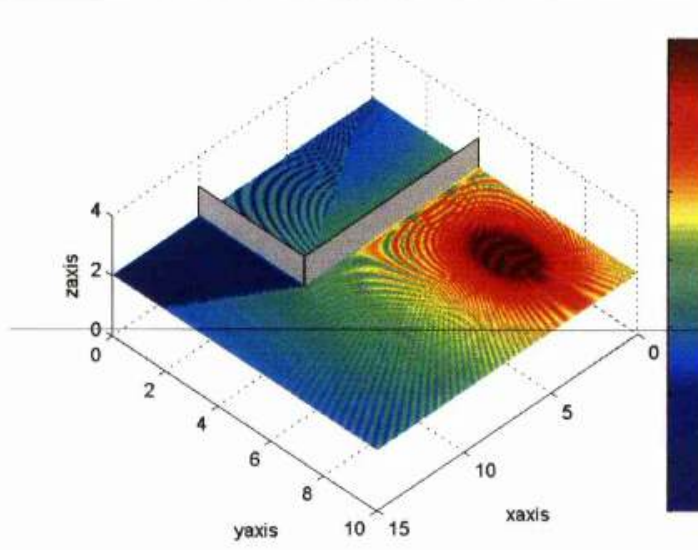

(a)

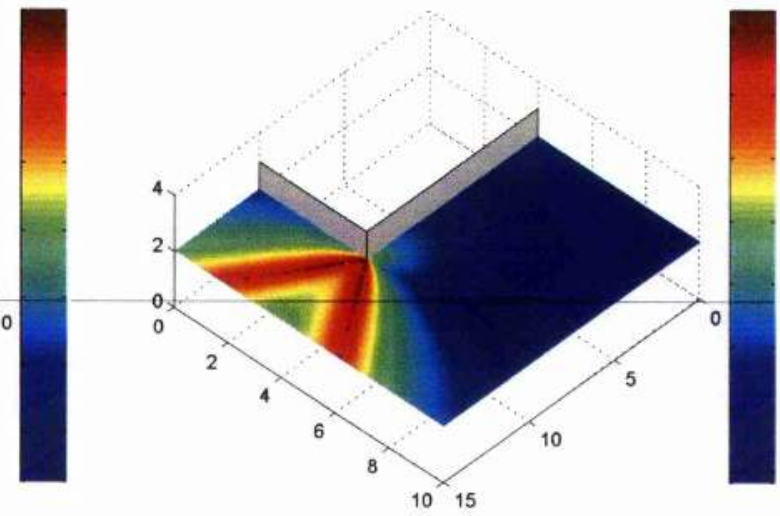

(b)

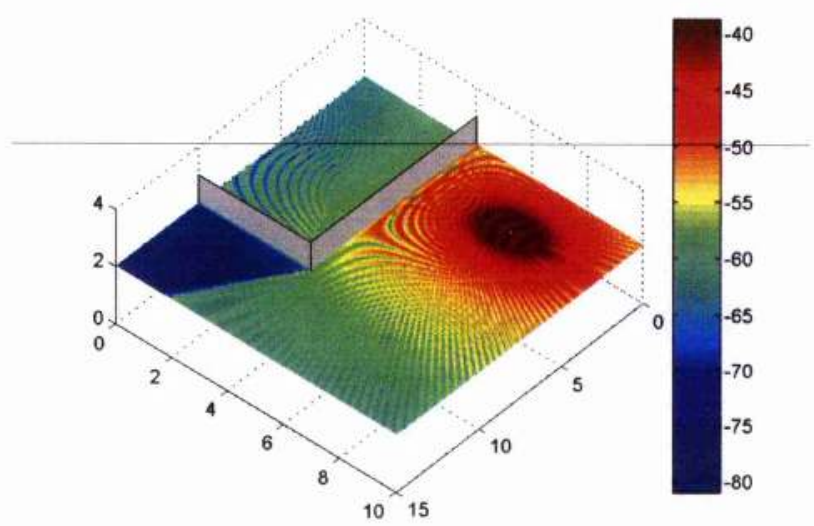

(c)

Figure 6-7: Diffraction effect. (a)Only reflection/refraction considered, (b) diffraction contribution, (c)reflection/refraction/diffraction considered

The transmission algorithm is called first to calculate the received field due to transmission through the environment's interfaces as if refraction was the only propagation mechanism. Reflection algorithm is called second, to calculate ray paths that undergo multiple reflections (maximum number of reflections is defined by the user) and all the associated intermediate transmissions. Diffraction is assumed to have significant effect in an indoor environment only if it 
is the first propagation mechanism that a propagating ray encounters. Incorporating multiple diffractions into the model, and especially considering that they occur between any other propagation mechanisms will make the model inefficient and very time consuming. No rigorous criterion for deciding what contributions to include in any simulation was used. The number and type of these contributions were empirically decided by observing the environmental description of the scenarios under investigation. For indoor environments that are dominated by reflections and refractions only a single UTD diffraction is incorporated using the formulation presented in section 3.2. Its modelled effect is presented in Figure 6-7.

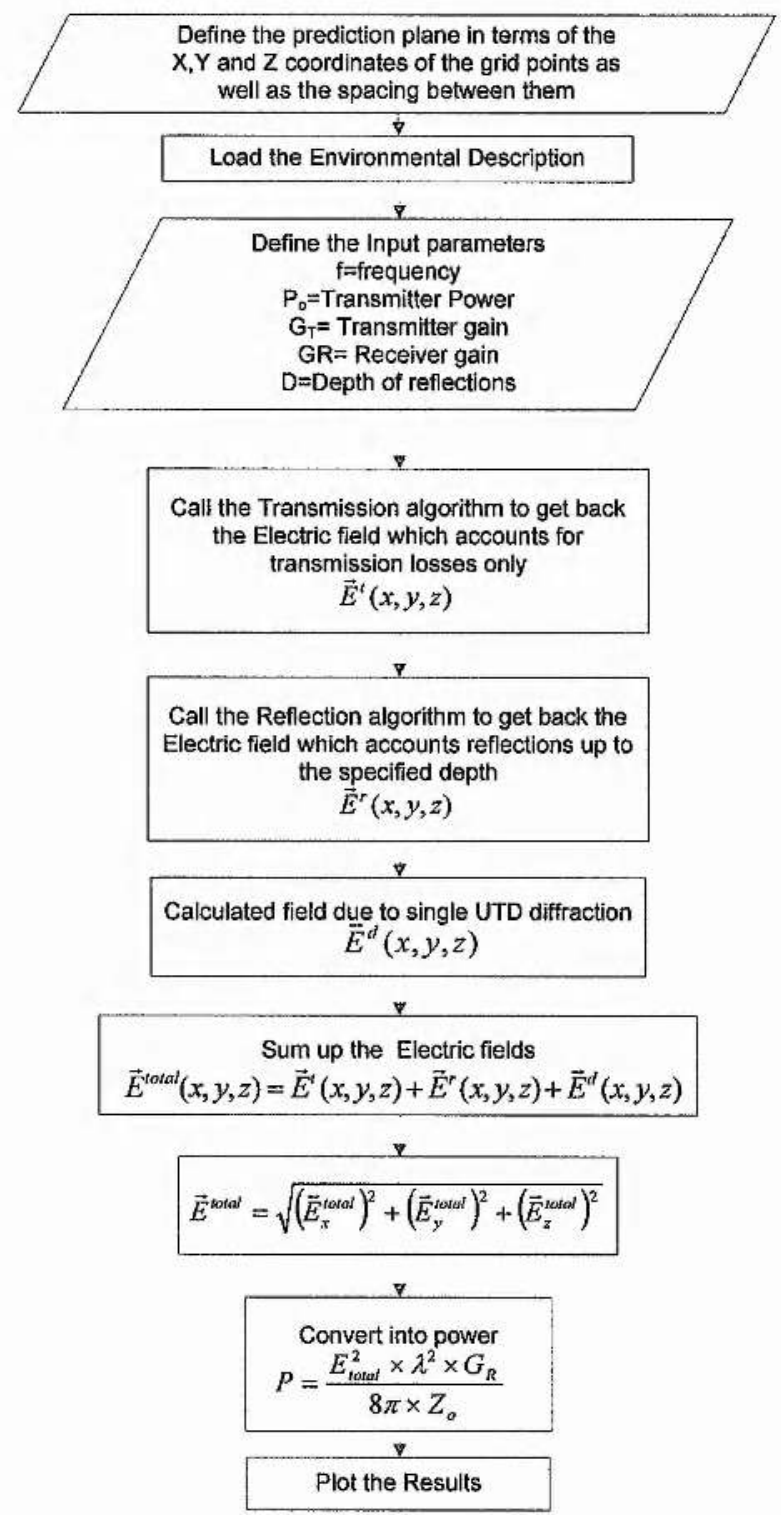

Figure 6-8: Final Model Algorithm 


\subsection{Basic Model Results and Validation}

In order to validate the basic Ray Tracing algorithm prior to the investigation of the effect of FSS, a real scenario (second floor of the Centre for Communication Systems Research, CCSR, University of Surrey) was simulated and radio measurements were performed on site. The basic Ray Tracing modelling approach was also compared with results obtained from a commercial Ray Tracing simulator by RemCom (Wireless Insite), which utilizes the SBR method (see section 3.4.1.)

\subsubsection{The CCSR}

The Centre for Communication System Research (CCSR) which is located in the northern part of the Surrey University Campus is a three storey building, with dimensions $45 \times 45 \mathrm{~m}$. The second floor, which is under investigation, has half the size of the lower ones, measuring at $25 \times 45$ $\mathrm{m}$ approximately. By using the CAD-plans of the $2^{\text {nd }}$ floor of the CCSR building (Figure 6-9) the environmental description of the model has been developed. The model environmental description comprises of 48 facets (internal or external walls) and 58 patches (windows or doors). This environmental description is shown in Figure 6-10.

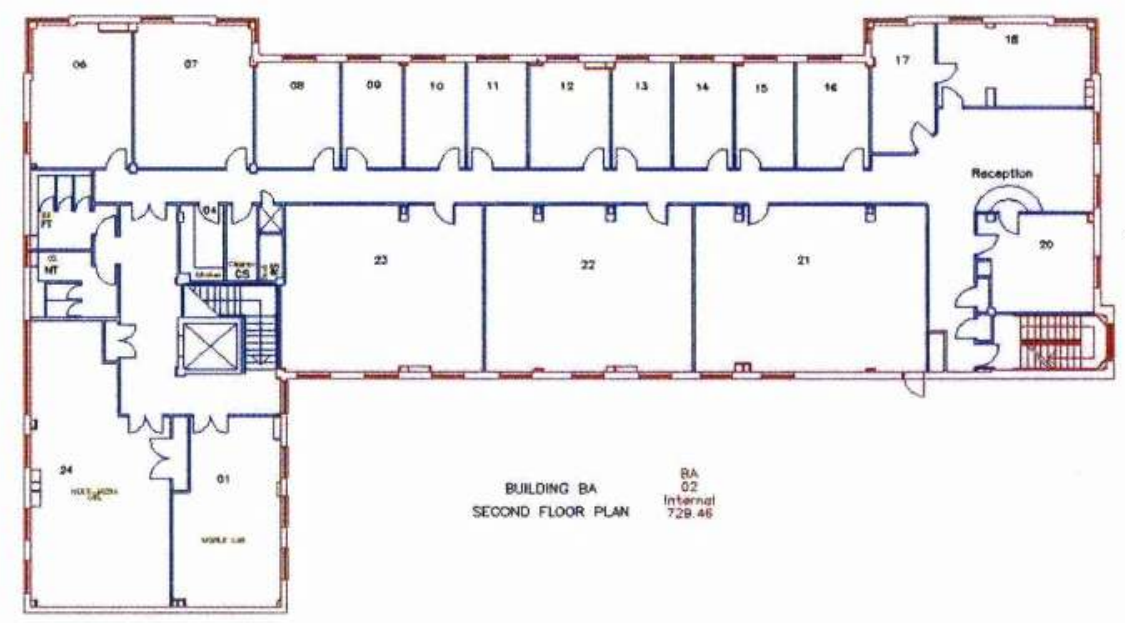

Figure 6-9: CCSR (2nd floor) CAD-plan

Detailed morphological description of the building's interfaces was not available, so conventional building materials were assumed. The external walls were assumed to be made of brick; floor and ceiling are made of concrete whereas the internal walls are made of plaster. The doors are assumed to be wooden and assumed to be always closed. The external double-glazed windows which are assumed not to have significant effect on the propagation prediction for the estimation route depicted in Figure 6-10, have been modelled as single-glazed because the ray tracing model uses a single layer model for calculating reflection and transmission coeffiencts 
(see section 2.4.1.2). The only metallic interfaces considered in the scene are the elevator ones. Typical constitutive parameters for different materials can be found in [52].

\begin{tabular}{|c|c|c|c|c|}
\hline Interface Type & Material & $\begin{array}{c}\text { Relative Electrical } \\
\text { Permittivity } \boldsymbol{\varepsilon}_{\mathbf{r}}\end{array}$ & $\begin{array}{c}\text { Conductivity } \\
\boldsymbol{\sigma} \text { (Siemens/m) }\end{array}$ & $\begin{array}{c}\text { Thickness } \\
\text { (meters) }\end{array}$ \\
\hline External Wall & Brick & 5.5 & 0.018 & 0.30 \\
\hline Floor \&Ceiling & Concrete & 8 & 0.01 & 0.40 \\
\hline Internal Wall & Plaster & 3.5 & 0.015 & 0.15 \\
\hline Door & Wood & 2 & 0.008 & 0.10 \\
\hline
\end{tabular}

Table 6-1: Electrical Parameters used for CCSR Interfaces

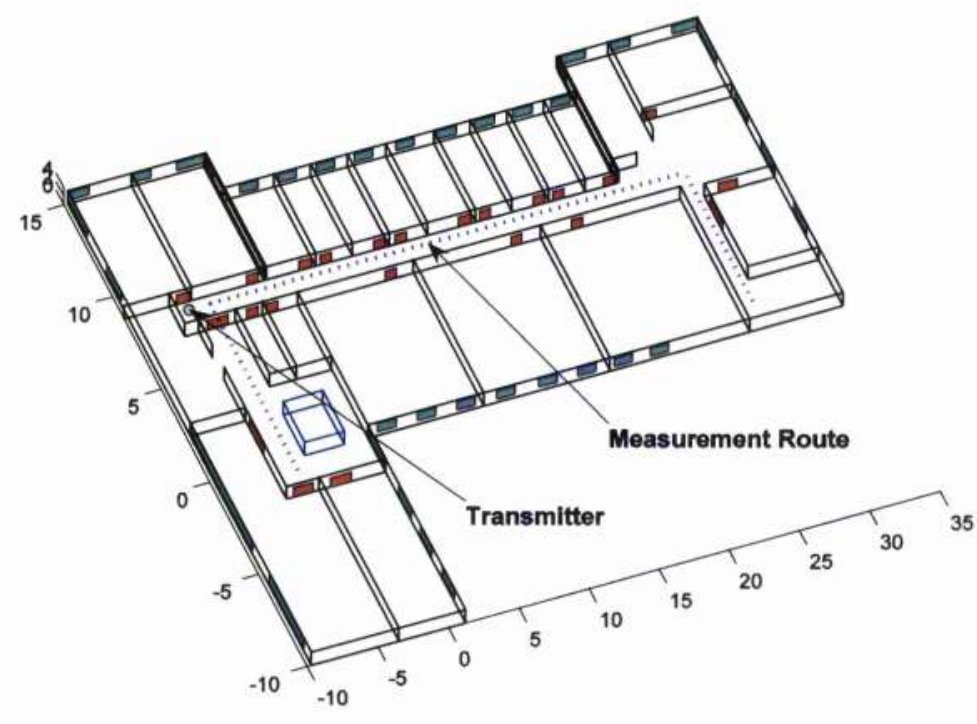

Figure 6-10: Environmental description of the $2^{\text {nd }}$ floor of the CCSR building

The WLAN system installed in the CCSR building consists of a number of wireless access points (Cisco Aironet 350 Series) which provide indoor-covered connectivity to wireless LAN terminals. It is based on the $802.11 \mathrm{~b} / \mathrm{g}$ protocol $(2.4 \mathrm{GHz}$ to $2.497 \mathrm{GHz})$ and offers data rates up to $54 \mathrm{Mbps}$. Typically the transmitting power of the access points can be configured between $1 \mathrm{mWatt}$ to $100 \mathrm{mWatts}$. The access points consist of a vertical dipole antenna with gain $2.2 \mathrm{dBi}$.

The developed model has been deployed to predict the radio coverage provided to the second floor of the CCSR building using installed transmitters. In order to cover the whole area a regular grid of observation points with spatial separation of 0.1 metres (which is less than the wavelength of operation: 0.125 metres) has been formed at height of 1 metre above the ground (this resulted into 113201 possible receiver locations). The transmitter is transmitting $5 \mathrm{mWatts}$ at 
$2.412 \mathrm{GHz}$ having $2.2 \mathrm{dBi}$ antenna gain. The receiver gain was assumed to be $0 \mathrm{dBi}$. Figure 6-11 shows the field prediction for this setup.

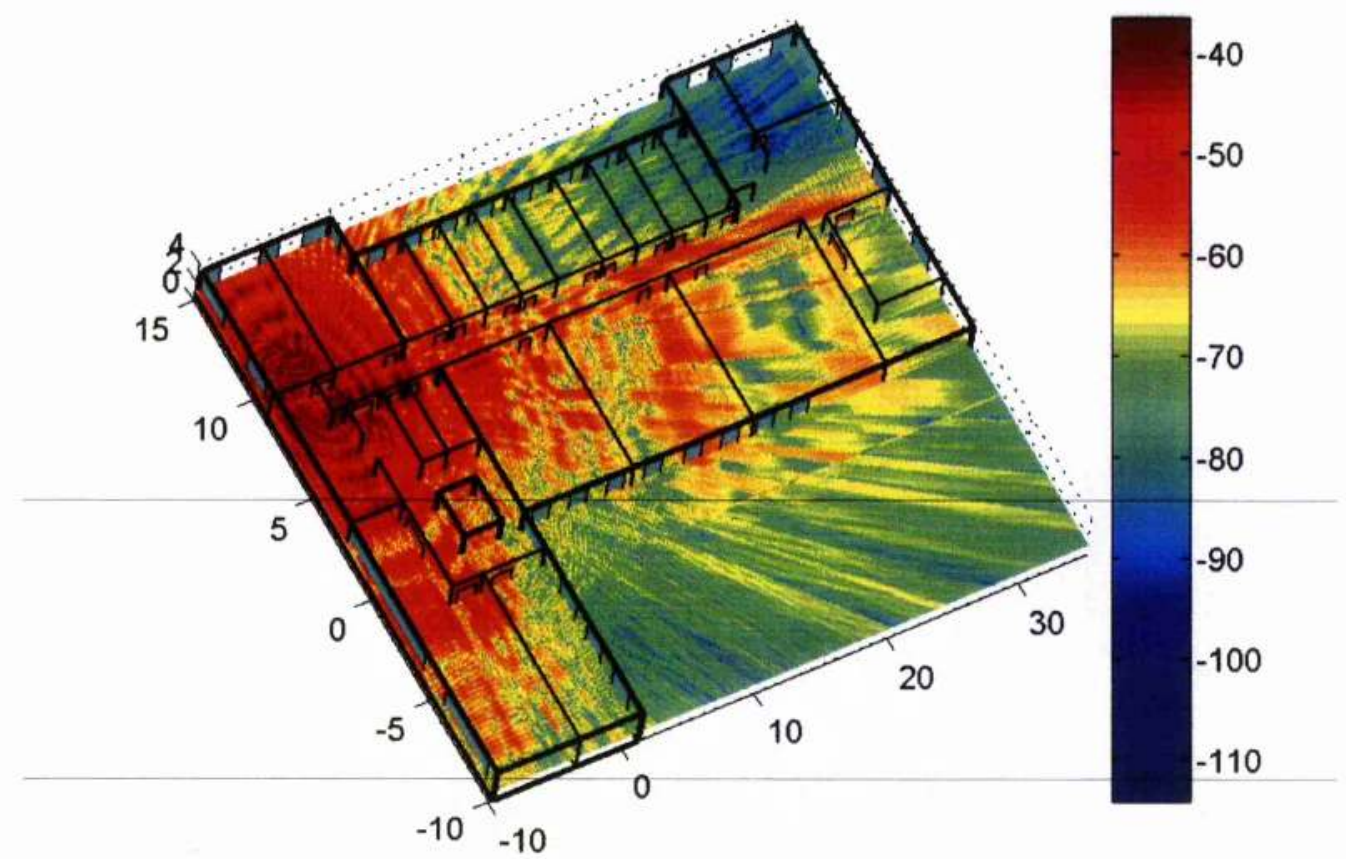

Figure 6-11: CCSR $2^{\text {nd }}$ floor field prediction at $2.412 \mathrm{GHz}$

\subsubsection{Model Validation}

To validate the model, measurements were performed along the measurement route depicted in Figure 6-10. The measurement route consists of 51 equally-spaced (both LOS and NLOS) averaged measurement points. Measurements were carried out with a portable spectrum analyzer from Rohde and Schwarz (FSP 30). The transmitter used was a calibrated Rohde and Schwarz signal generator transmitting a $10 \mathrm{dBm} \mathrm{CW}$ signal at a frequency of $2.4 \mathrm{GHz}$. Dipole antennas were used for transmitting and receiving. In order to account for fast fading variations, for every measurement point the receiver was slightly shifted by few wavelengths $(25 \mathrm{~cm})$ and the average of all the corresponding measurements was taken and compared with the model prediction for that particular point. Model predictions were obtained considering $2 \mathrm{~cm}$ spacing between the individual receiver positions, in order to capture fast fading effects. However, since model prediction provides exact phasor total fields and not local averages, a sliding filter has been used to average them over a window of two wavelengths. A single diffraction, three reflections and all the possible transmissions have been considered for this simulation. Figure 6-12 shows the comparison of the theoretical predictions using both the developed model and Wireless Insite and 
the measurement set. The recorded set of measurements has indicated a mean square error of around $4.15 \mathrm{~dB}$ and a model error standard deviation of around $5 \mathrm{~dB}$ which are considered acceptable. Various reasons exist for the small differences between the predictions and the measurements and most of them are explained in section 3.5

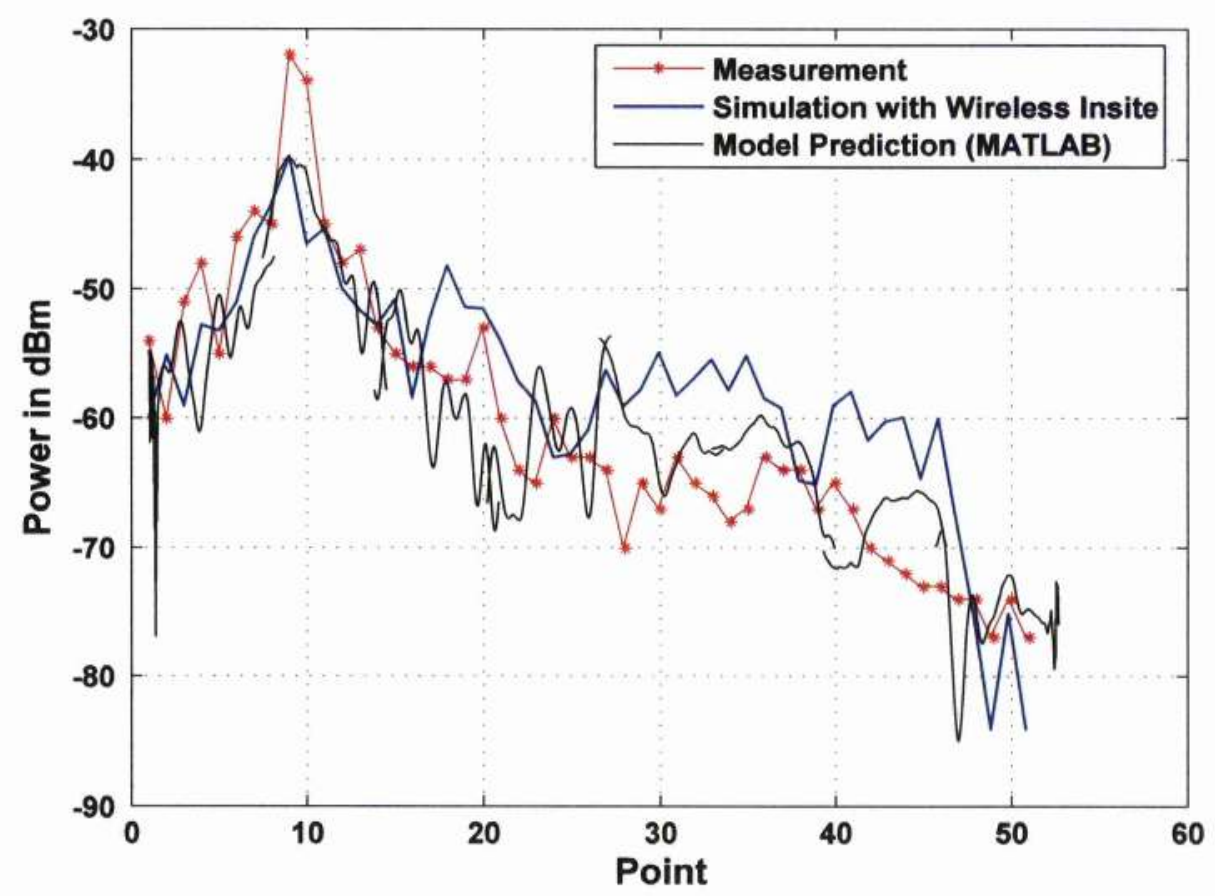

Figure 6-12: Comparison between measurements and modelling

\subsection{The Ray-Tracing FSS model}

Once the basic Ray-Tracing model was verified, it was enhanced in order to include the theoretical FSS behaviour. As it can be seen from Figure 6-1 the .Facet structure in the model environment description contains a flag parameter .fss which indicates whether an FSS is attached to any of the scene's facets. If there is an FSS, then when a ray interacts with that particular Facet, the model does not follow the conventional procedure of calculating the Fresnel coefficients discussed in section 2.4.1, but instead it loads a set of pre-calculated angle-dependent reflection and transmission coefficients for the FSS-facet combination, obtained using the CFDTD method presented in section 5.2.3.1. Typical FSS reflection and transmission coefficients for various angles of incidence have been presented in Figure 5-14. To incorporate the three-dimensional scattering behaviour of any deployed FSS, the CFDTD reflection and transmission coefficients are pre-calculated for various elevation and azimuth angles $(\varphi$ and $\theta$ respectively as shown in Figure 2-10 with the FSS-wall structure lying on the $x-y$ plane). 


\subsubsection{Model Validation}

In order to validate the developed model and verify the applicability of Ray-Tracing in predicting radio propagation from FSS indoor environments, a small-scale indoor environment has been constructed and measured in an anechoic chamber as depicted in Figure 6-13a.

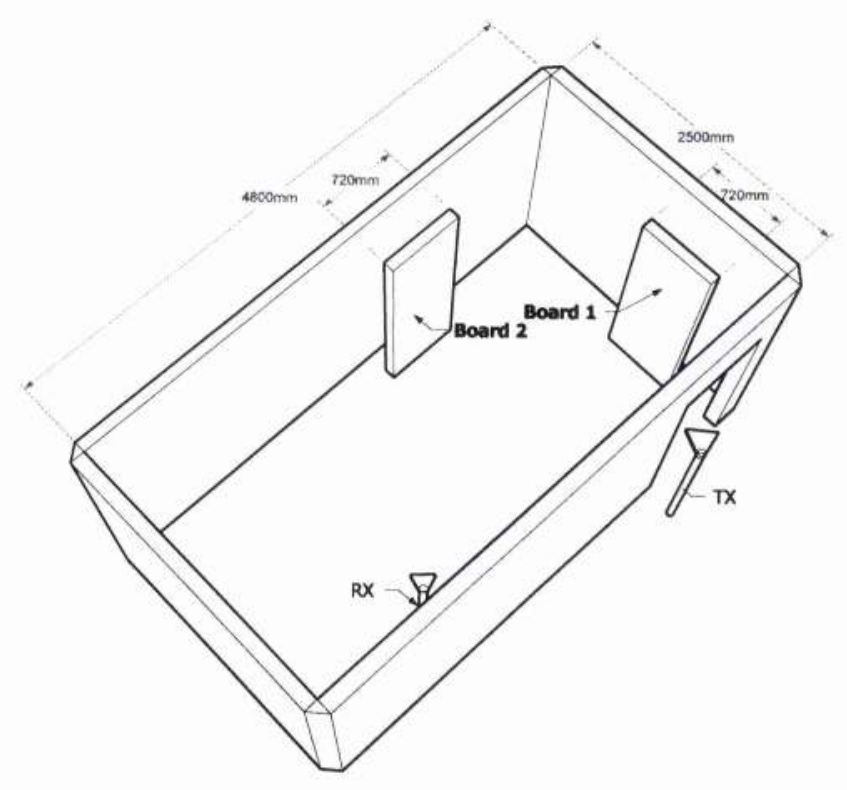

(a)

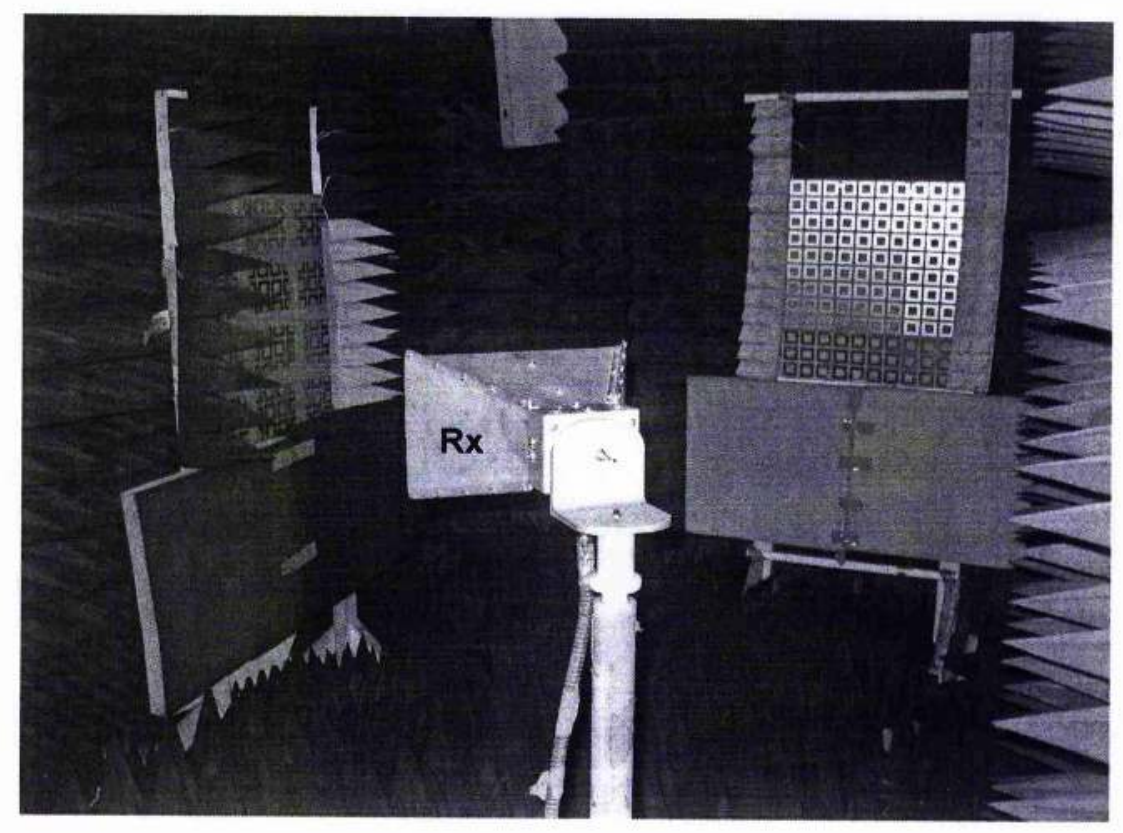

(b)

Figure 6-13: Anechoic Chamber Experiment Setup (internal view) 
The transmitting antenna was placed outside the chamber in such a way so as a LOS component could not be received by the identical receiving antenna $(1-8 \mathrm{GHz}$ horn antennas with 45 degrees beamwidth - see Appendix 1), which was placed inside the chamber. Two wooden boards were placed inside the chamber to direct the ray components to the receiving antenna (Figure 6-13b). With this setup, the ray launched by the transmitter reflect on board 1 and 2 and then reach the receiver. The distance travelled by the ray after the two reflections is 6.3 meters, corresponding to a path loss of $56 \mathrm{~dB}$ at $2.4 \mathrm{GHz}$. The angle of incidence on the first board is $45^{\circ}$ and $54^{\circ}$ on the second. The theoretical CFDTD frequency responses of the square loop under these two angles of incidence have been incorporated into the Ray Tracing Model. The anechoic chamber is covered with Emerson and Cumming pyramidal shaped, carbon loaded urethane foam absorbers with very low reflectivity. For frequencies between $1-4 \mathrm{GHz}$ these absorbers can present 40-50 dB of reflection loss at various angles of incidence. This effectively means that any reflected contributions from the anechoic chamber walls will have insignificant effect on the measured response.

Three cases have been measured; reflectors (1) are made of wood, (2) covered by metallic surfaces and (3) by FSS as depicted in Figure 6-13b. For the purpose of this experiment a squareloop FSS tuned at $2.4 \mathrm{GHz}$ was designed and fabricated on FR4 dielectric $\left(\varepsilon_{\mathrm{T}}=4.55, \tan \delta=0.0175\right)$. Figure 6-14 presents the theoretical behaviour (CFDTD) of this square-loop FSS under various angles of incidence.
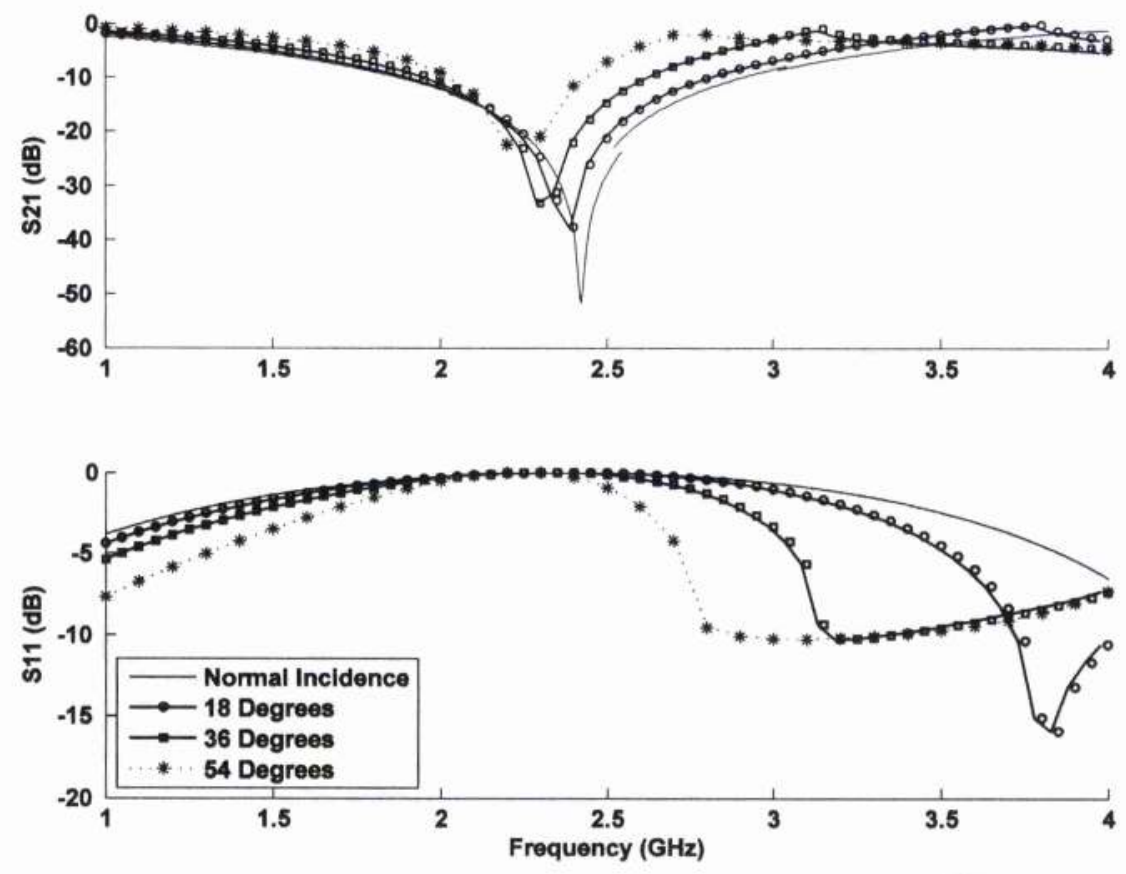

Figure 6-14: Transmission (S21) and Reflection (S11) coefficients of a square-loop FSS under various angles of incidence as suggested by the CFDTD method. 
Figure 6-15 presents the results of these measurements. It is clear that there is a significant increase of the field strength at $2.4 \mathrm{GHz}$ for the FSS case compared to the results obtained from the wooden board case. The received power at $2.4 \mathrm{GHz}$ is the same as the one for the metallic case, but significantly lower for any other frequencies. This result verifies the application of the frequency selective surface as a passive repeater, meaning it can be used to increase signal coverage on specific frequencies without affecting the operation of systems operating on other frequencies. Also this experiment and the comparison with the theoretical ray tracing results demonstrates that ray tracing can be applied to predict radio propagation in FSS indoor environments.

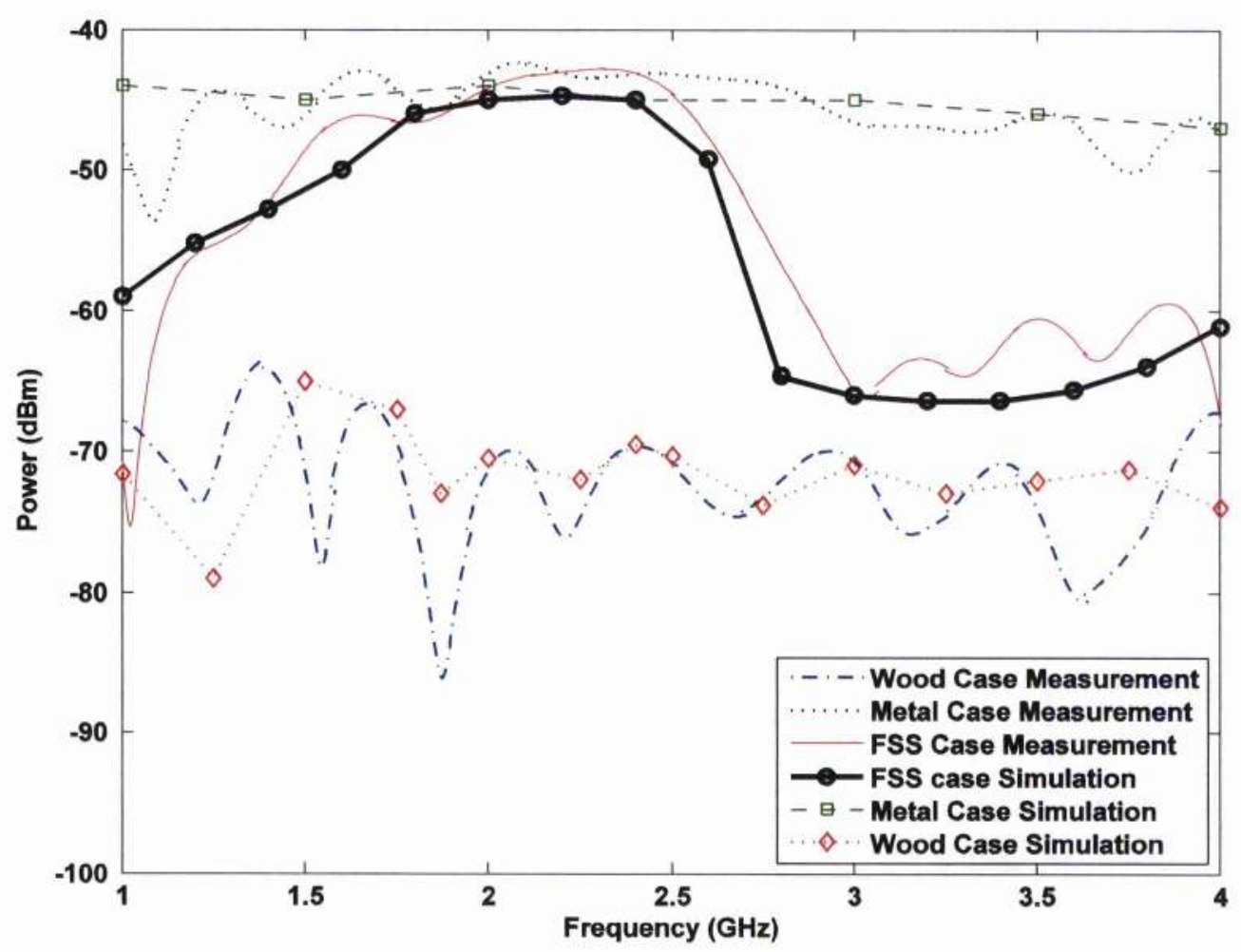

Figure 6-15: Anechoic Chamber results for the 3 cases under investigation

\subsection{Summary}

The applicability of Ray Tracing for predicting radio propagation in FSS environments has been demonstrated in this chapter through the use of a custom written Image-method algorithm. The model was modified to incorporate the theoretical behaviour by replacing the Fresnel reflection and transmission coefficient calculations with pre-calculated (CFDTD) angle-dependent (three dimensional) FSS coefficients. 
Basic model predictions from a real environment ( $2^{\text {nd }}$ floor of the CCSR) have been compared with results obtained from a commercial simulator (Wireless Insite by RemCom) and with measurements carried out on site. Comparison indicated a reasonably good agreement. The FSS-Ray Tracing model was also validated through a small scale indoor FSS environment constructed and measured in a controlled environment (anechoic chamber).

The developed tool was used to study the effect of incorporating FSS in indoor environments. Results of this study are presented in the next chapter. 


\section{Chapter 7}

\section{Effect of FSS in Wireless Environments}

The modified Ray-Tracing model presented in the previous chapter was utilised to study the effect of deploying Frequency Selective Surfaces in wireless environments. The study was concentrated to WLAN $(802.11 \mathrm{a} / \mathrm{b} / \mathrm{g})$ frequencies $(2.4 \mathrm{GHz}$ and $5.2 \mathrm{GHz})$. The fundamental idea was to utilise Frequency Selective Surfaces in order to,

1. Achieve frequency selective radio coverage improvement in areas of interest. This is based on the assumption that the mean signal due to a reflected contribution arising from a Frequency Selective Surface would be stronger than the one received from any other non-metallic object.

2. Provide isolation by selectively rejecting a frequency range, thus reducing interference or increasing wireless security by minimising the spill-over of radio waves outside designated areas.

3. Guide the wanted radio signals to areas of limited signal coverage by utilising FSS and the internal structure of the physical environment.

4. Achieve all the above goals without affecting the operation/radio propagation characteristics of any other system operating in the close vicinity of the targeted system.

This chapter presents Ray-Tracing simulations for various indoor scenarios presenting how frequency selective surfaces can be utilised as passive repeaters to achieve the above objectives. It has to be noted that for the presented simulation results, various factors governing the FSS propagation characteristics such as angle of incidence, polarisation as well the interaction of FSS with the building materials (as presented in section 5.3) have been taken into account. An initial flavour of the effect of deployed FSS on the potential capacity increase from using a MIMO system is demonstrated. 


\subsection{Initial Investigation-Feasibility Study}

In order to demonstrate the principle and the feasibility of the application proposed some initial investigation has been carried out [101]. This included simulations in a simple theoretical indoor scenario consisting of 14 brick-30cm-thick walls (including floor and ceiling) with relative permittivity $\varepsilon_{r}=5$ and conductivity $\sigma=0.1$. In order to get an initial idea of the effect of FSS when these are incorporated in an indoor wireless environment, a square-loop design (tuned at $2.4 \mathrm{GHz}$ ) was assumed to replace the actual walls that the FSSs were deployed on.

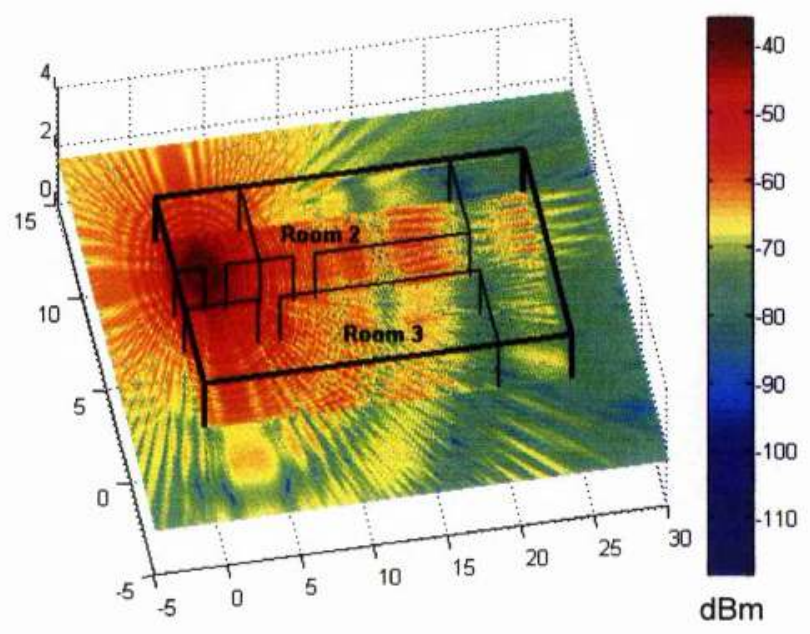

Figure 7-1: Field Prediction at 2.4GHz without FSS

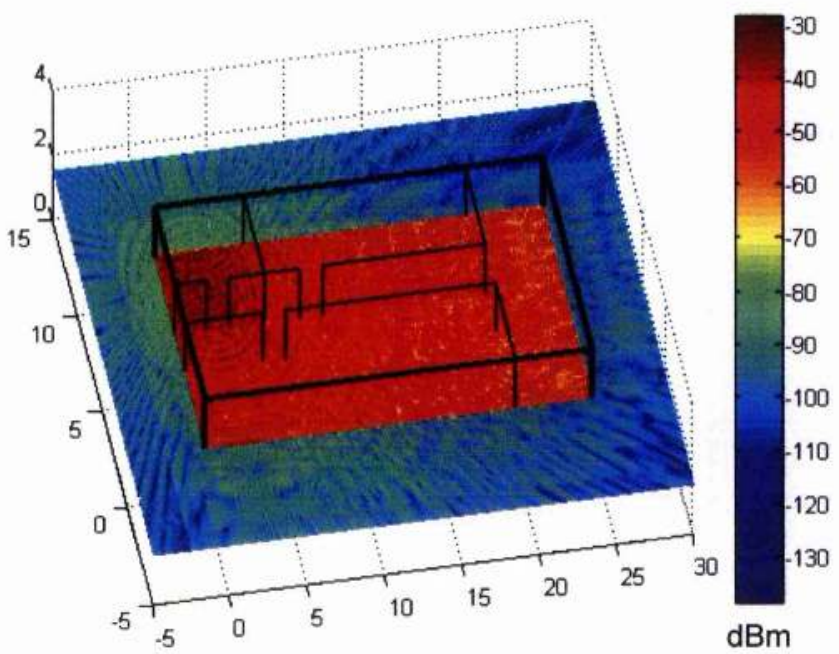

Figure 7-2: Field Prediction at $2.4 \mathrm{GHz}$ with FSS replacing the external walls only 
Figure 7-1 and Figure 7-2 clearly demonstrate the feasibility of the aforementioned objectives 1 and 2. It is clear that by replacing the external walls with FSS structures, signal coverage is generally increased providing higher system reliability. At the same time, the signal outside the building has greatly decreased, reducing possible interference from this system to any other network that might operate on the same or very close channel frequency. Figure 7-3 shows the field prediction obtained at $2.4 \mathrm{GHz}$ when FSS are assumed to replace the external walls as well as the walls of Room 2 and Room 3 as depicted in Figure 7-1. The doors are assumed to be closed and also replaced by FSS. It can be observed that the signal coverage in Rooms 2 and 3 is significantly reduced while the corridor acts as a waveguide, guiding the signal to other areas.

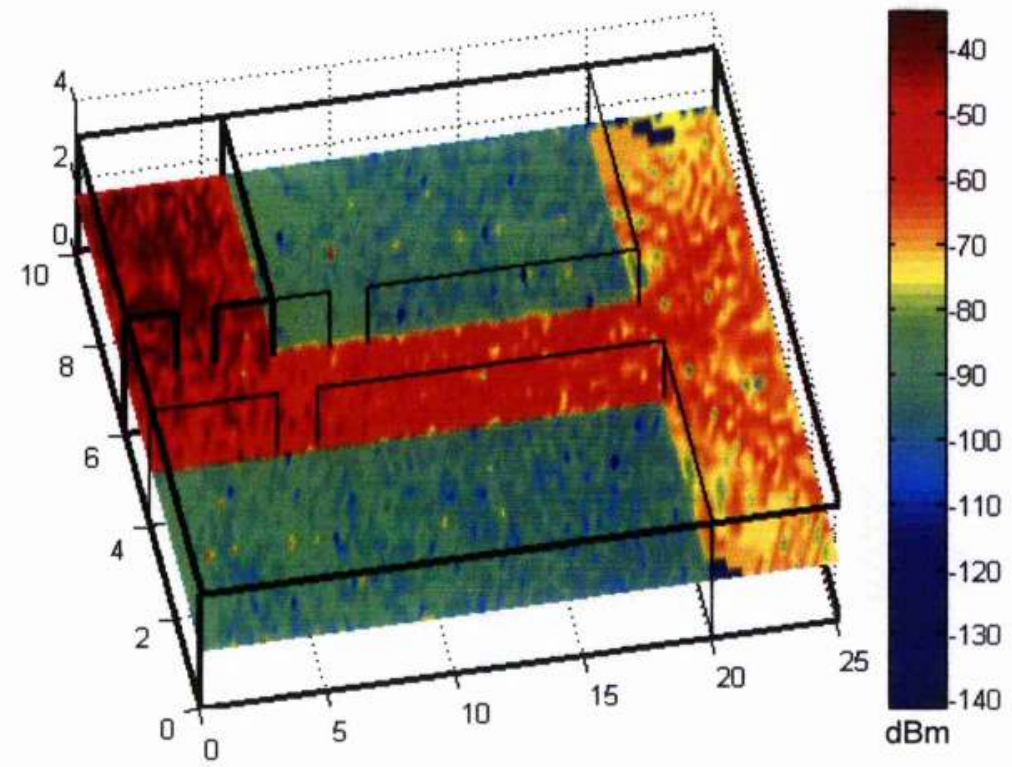

Figure 7-3: Field prediction at 2.4GHz with FSS added on external and internal walls

This initial study through indicative coverage plots, has verified the feasibility of utilising FSS as passive repeaters and/or isolators in wireless environments. The frequency selectivity of this application is going to be demonstrated in subsequent scenarios where more results from various FSS-placement combinations are presented as well.

\subsection{Scenario 1}

The first scenario into investigation shown in Figure 7-4 is a simplified indoor environment with dimensions 2232 metres [102]. The external walls are assumed to be made of brick where as the internal ones are assumed to be made of plaster. The floor and ceiling are made of concrete. Typical constitutive parameters of these materials (see Table 6-1) have been used and all the 
interfaces were assumed to be homogeneous. The facets in the simulated environment where also described in such a way as to account for the theoretical and measured FSS behaviour under various angles of incidence as suggested by the CFDTD method and verified through anechoic chamber measurements.

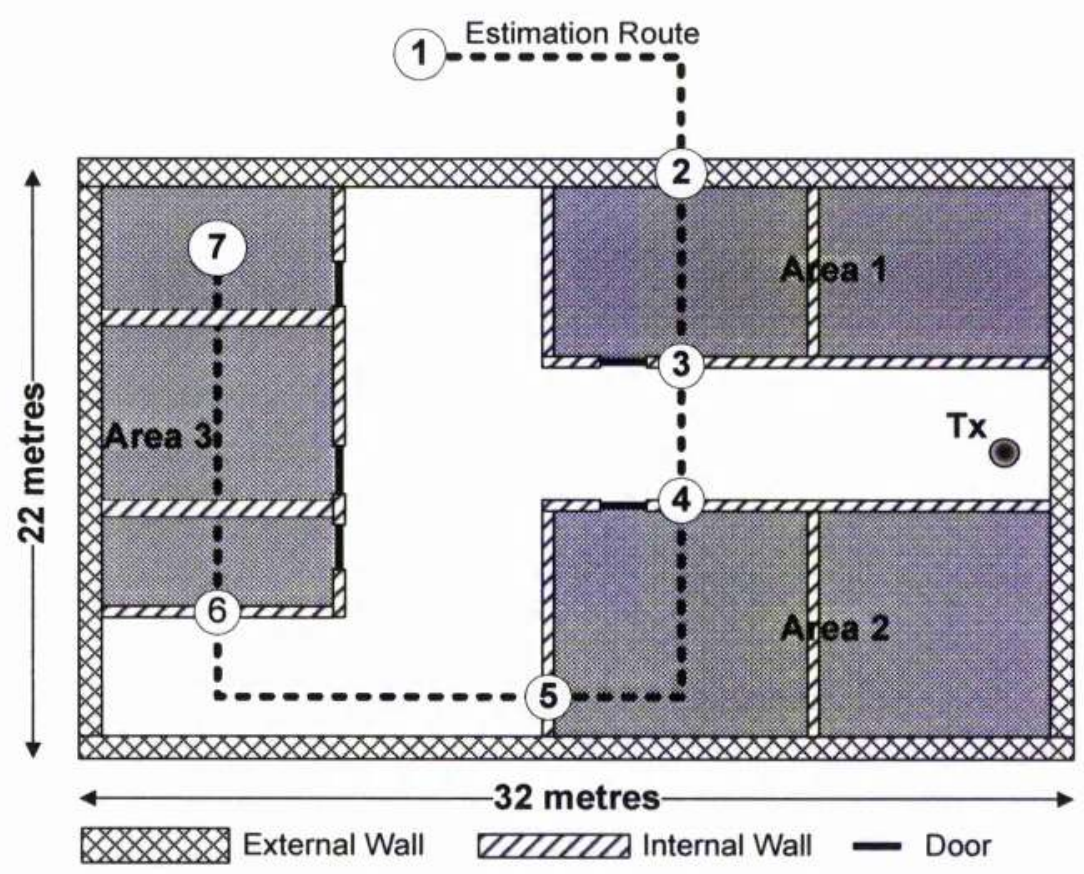

Figure 7-4: Scenario 1

A square loop FSS design has been chosen to be deployed on the environment's interfaces mainly due to its superiority against other FSS designs in terms of its angular insensitivity as presented in Table 5-1[70]. The square loop was designed on FR4 material $\left(\varepsilon_{\mathrm{r}}=4.55, \tan \delta=0.0175\right)$ with a $2.4 \mathrm{GHz}$ tuning frequency. The dimensions of square loops are shown in Figure 7-5. The CFDTD method was used to calculate the reflection and transmission coefficients for the FSS-airgap-building wall combination. As already verified through simulations and measurements in section 5.3, when the airgap between any FSS and any building material is bigger than $\lambda / 10$, then there is no significant effect on the FSS tuning characteristics. For this reason, the square loop FSS was placed $12.5 \mathrm{~mm}$ away from all building materials (i.e. brick, plaster board, wood and concrete). An illustrative example is shown in Figure 7-6 where the square loop FSS is placed $12.5 \mathrm{~mm}$ away from plaster board. 


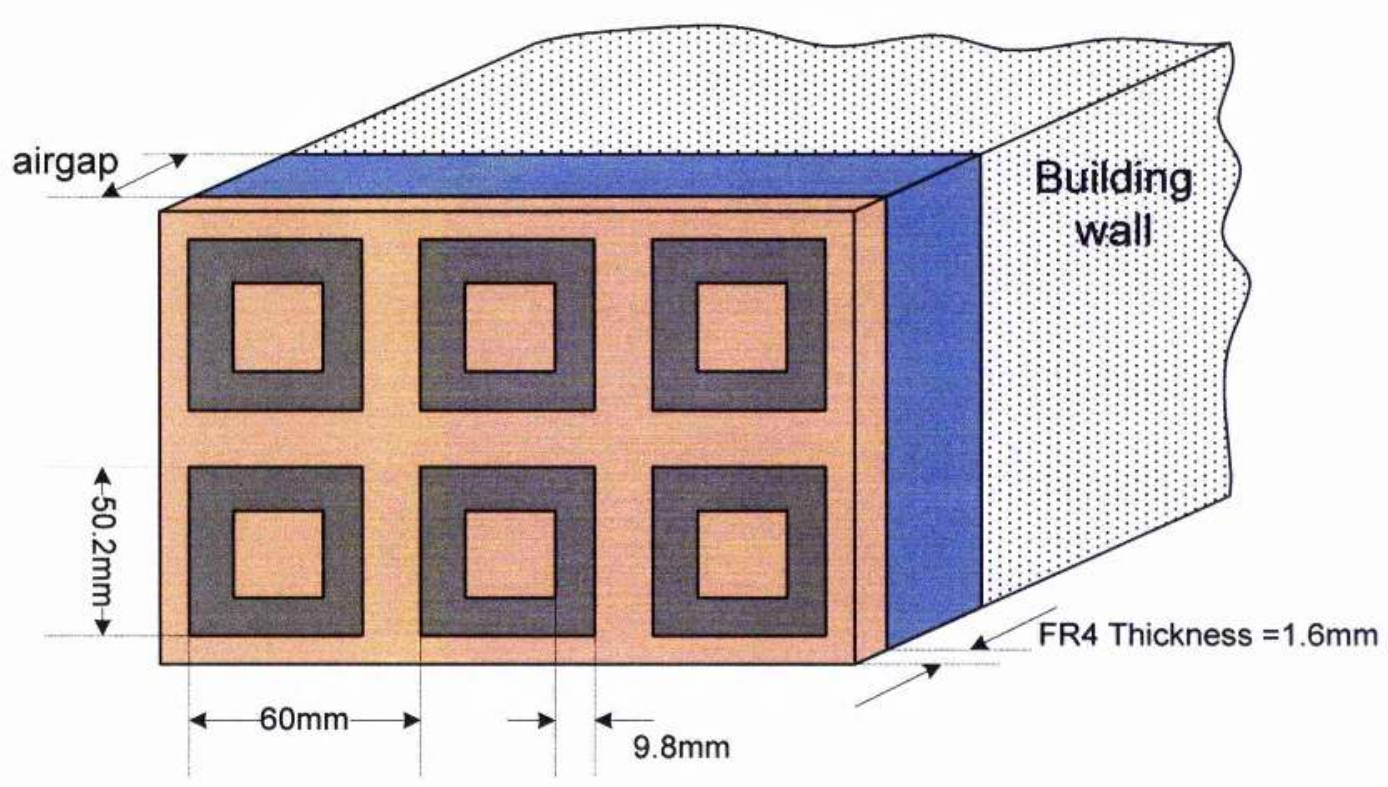

Figure 7-5: Square loop FSS tuned at $2.4 \mathrm{GHz}$ deployed on building walls

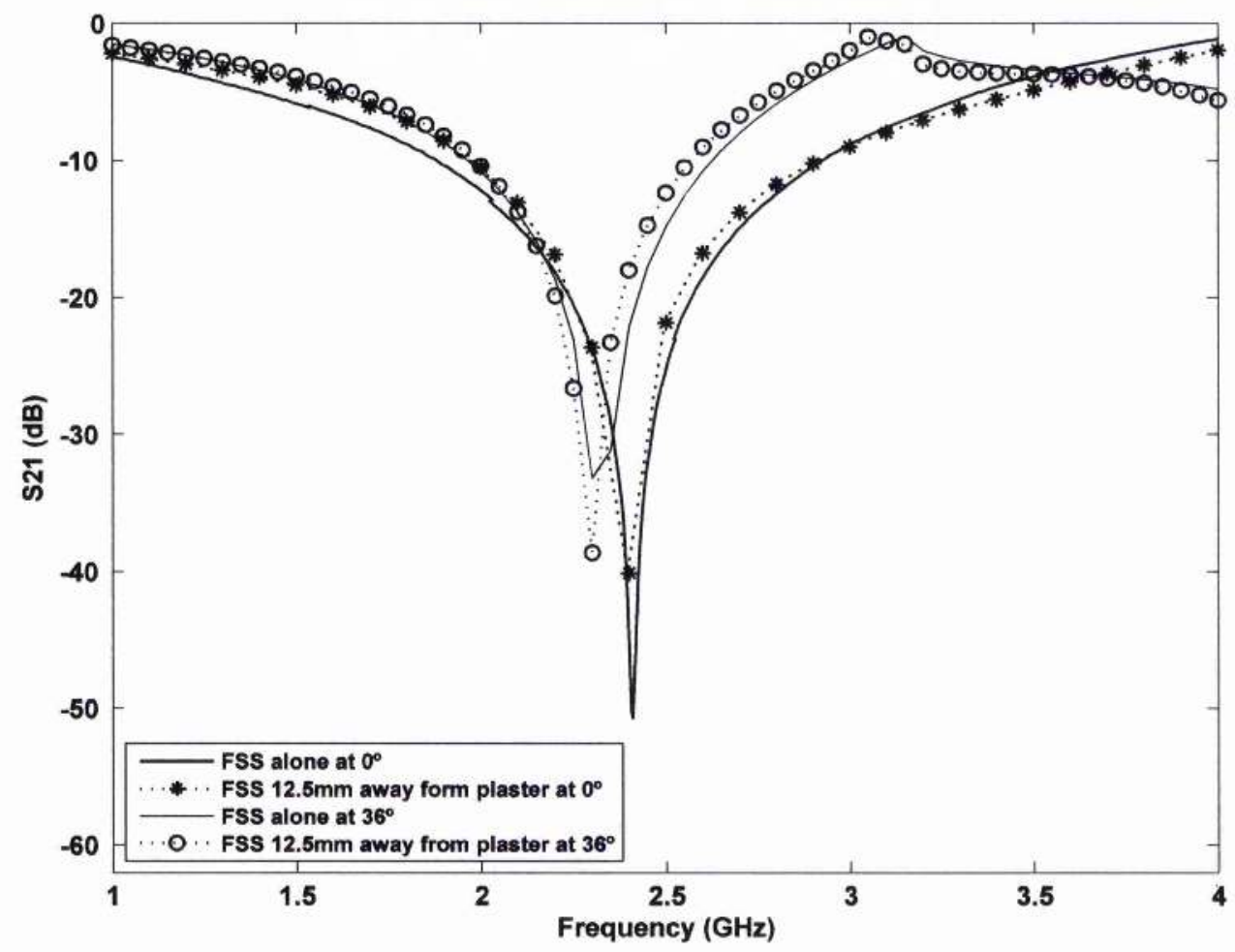

Figure 7-6: Transmission loss for square loop FSS free standing case and the case where it is placed $12.5 \mathrm{~mm}$ in front of plaster. 
Firstly the effect of deploying the FSS, on the four external walls was examined. RayTracing simulation results obtained along the estimation route depicted in Figure 7-4 are shown in Figure 7-7. The spacing between the receiver locations along this estimation route is 0.05 metres. The results shown in Figure 7-7 have been averaged (using a sliding averaging filter) in order to remove fast fading.

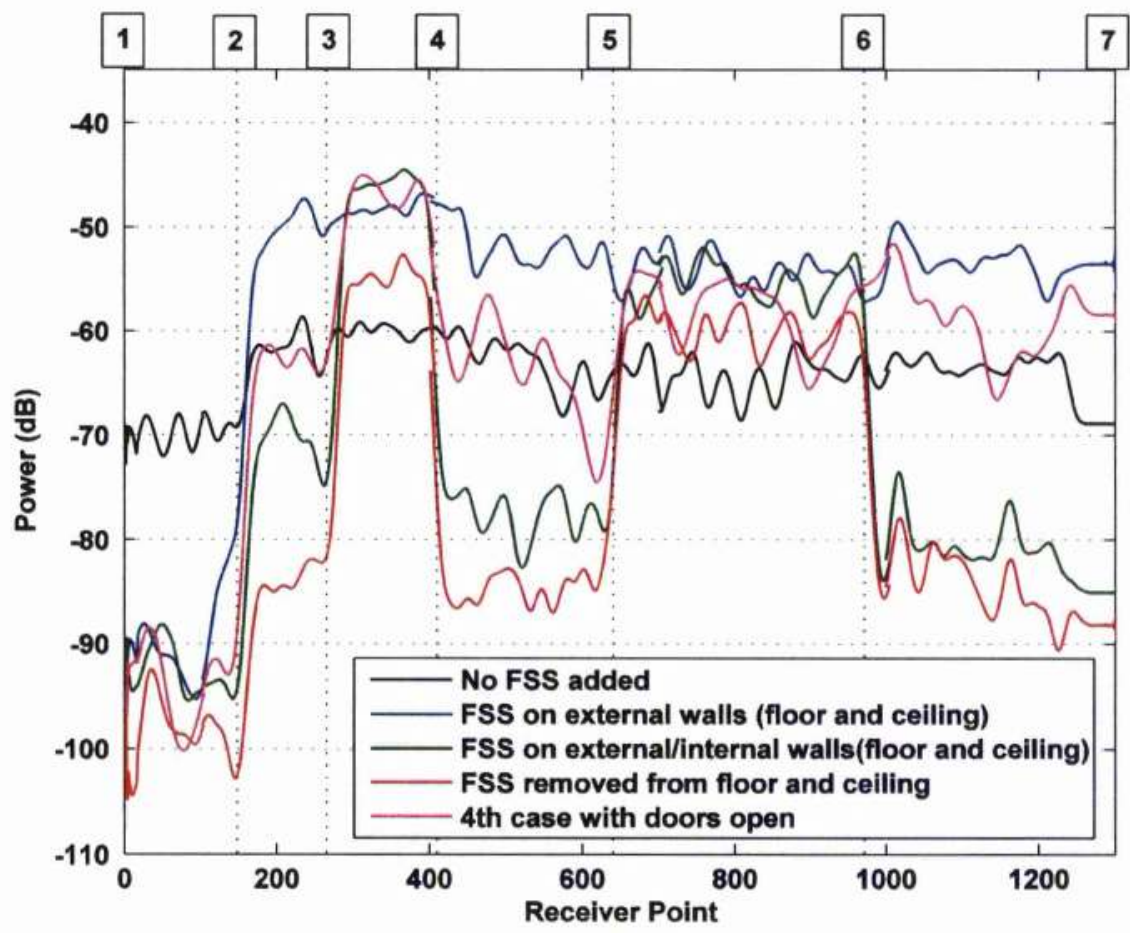

Figure 7-7: Comparison of different cases at $2.4 \mathrm{GHz}$.

It is obvious that the field strength inside the building is increased where as the one outside the building is decreased. The power outside the building is reduced by roughly $20 \mathrm{~dB}$ which effectively reduced the interference to any other wireless networks operating at the same or similar frequency outside the building. The power inside the building is increased by roughly $10 \mathrm{~dB}$.

The next step into the investigation was to find a way to channel the signal along the corridor, whereas at the same time restrict it from areas 1, 2 and 3 as shown in Figure 7-4. In this case, FSS were deployed on the internal walls as well. Results in Figure 7-7 (green line) suggest that there is significant increase of the field strength along the corridor, compared to the non-FSS case (black line) of around 10-12dB. Also, there is around 10-15dB field reduction in areas 1,2 and 3, again compared to the non-FSS case. Someone would expect that this reduction would have been $20 \mathrm{~dB}$ as in the case of the FSS deployment on the external walls only. This does not happen because the high attenuation suffered by the rays while crossing the internal walls is 
compensated by the stronger reflection (and effectively less attenuation) coming from the floor and ceiling where FSS is also deployed. In order to verify this, the floor and ceiling FSS were then removed. The results (red line in Figure 7-7) indicate a further field reduction in the areas of interest but at the same time a slight reduction of the field strength along the corridor, compared to the previous case (green line) which is due to the absence of the floor and ceiling strong reflections.

All the above cases have assumed that the doors shown in Figure 7-7 were closed and covered with FSS. The case where FSS are added on external and internal walls (including floor and ceiling) has been re-simulated assuming that the doors where left open. The results (pink line in Figure 7-7) indicate that the corridor still acts as a waveguide providing better coverage in the region between points 5 and 6 compared to the non-FSS case. However, there is significant spill over of radio waves inside areas 1,2 and 3 effectively reducing the required coverage isolation in these areas. This means that, in order to achieve high coverage isolation in certain regions of the environment, doors (or even windows) that can act as source of spilled over rays into those regions need to be covered with FSS and remain closed.

These results indicate that with different FSS arrangements inside the environment, different objectives (coverage restriction, field increase or wave guiding effects) can be achieved, based on the designers' wish.

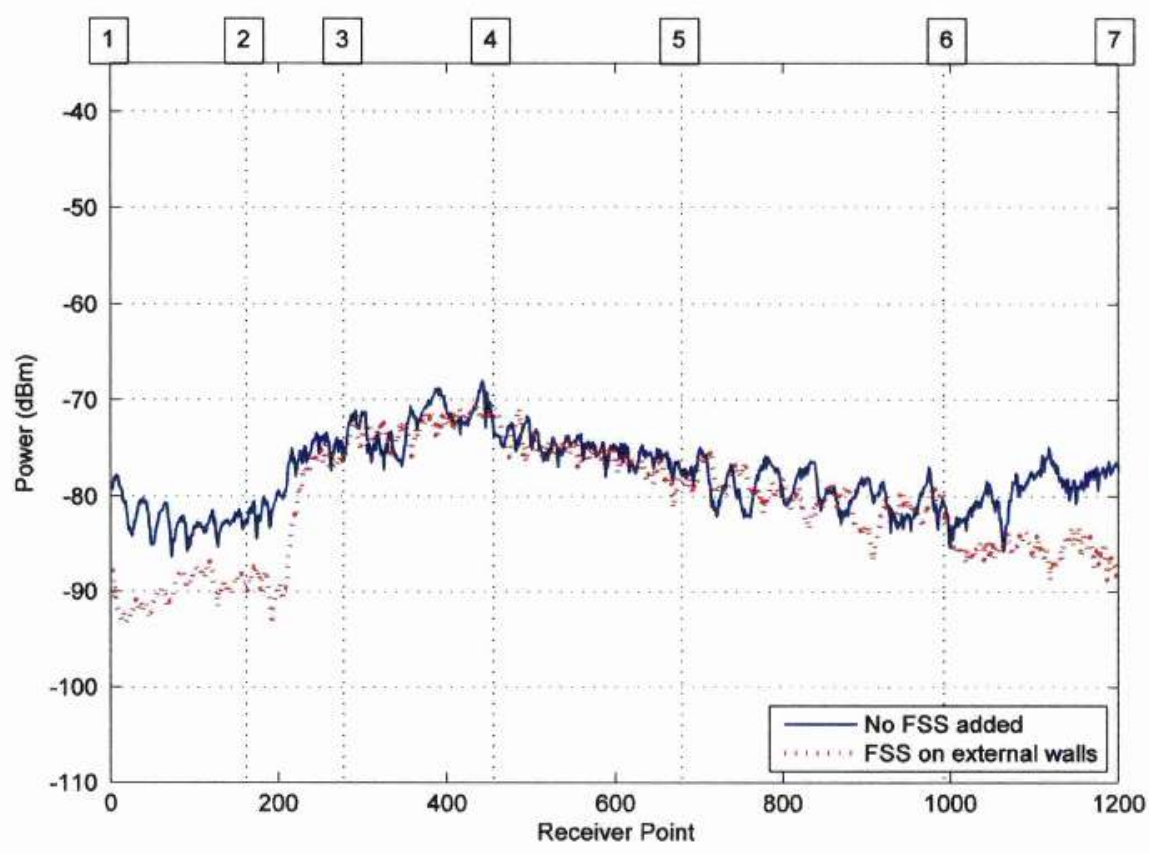

Figure 7-8: Effect of a $2.4 \mathrm{GHz}$ tuned FSS on a $5.2 \mathrm{GHz}$ wireless system (scenario 1) 
To highlight the effect of a deployed FSS, tuned at $2.4 \mathrm{GHz}$ on a different frequency, a radio transmission at $5.2 \mathrm{GHz}$ was assumed. The transmission and reflection coefficients at 5.2 $\mathrm{GHz}$ were again obtained using the CFDTD method. Figure 7-8 suggests that the FSS has very little effect on the radio propagation characteristics at this frequency since the FSS is not tuned at $5.2 \mathrm{GHz}$. This effectively means that a carefully designed and deployed FSS will not have any effect on a system operating on another frequency. A specific example could be the confinement of WLAN signals within a building without obstructing the transmission of cellular signals through such a frequency selective building.

\subsection{Scenario 2: Typical Office Floor}

Scenario 1 has achieved all the objectives mentioned in the beginning of this chapter. However, it would be wiser to simulate a different environment in order to verify the generality of the proposed application. For this reason a typical office floor environment (Figure 7-9) has been defined and simulated [103], utilising the square loop FSS presented in the previous section. The office floor consists of 5 offices and a large meeting room located away from the access point (Tx) position. The idea in this environment is to utilise the corridor as a frequency selective waveguide to channel the signals and effectively increase the coverage in the meeting room.

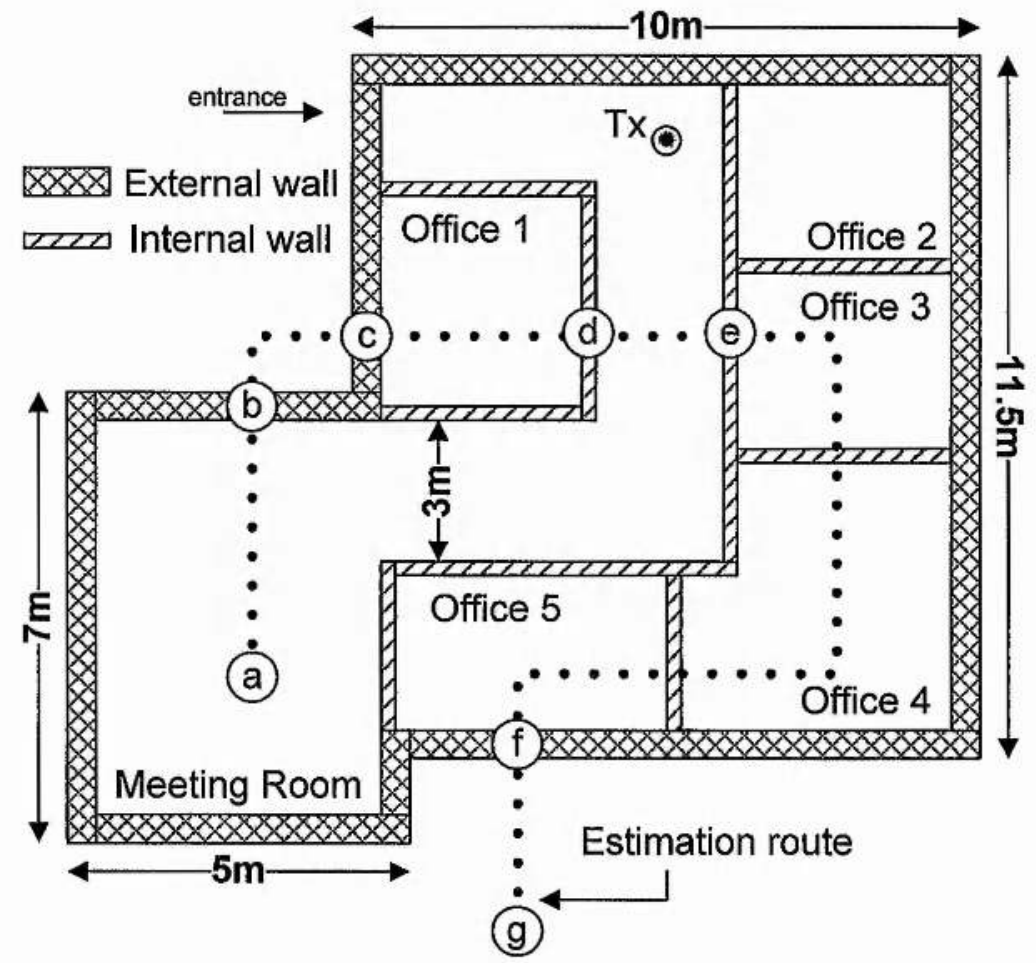

Figure 7-9: Scenario 2. Typical Office floor 
Following the same methodology as in the first scenario, FSSs have been initially used on the external walls only (including floor and ceiling). Results obtained along the depicted (in Figure 7-9) estimation route are shown in Figure 7-10. The spacing between the receiver locations along the estimation route is 0.10 meters. Two dipole antennas with gain equal to $2.15 \mathrm{dBi}$ have been used as transmitting and receiving antenna elements. The transmitting power was set to 10 $\mathrm{dBm}$. Similarly to scenario 1 , the obtained results suggest that the field strength inside the building has generally increased by roughly $10-20 \mathrm{~dB}$ where as the one outside the building has been reduced by $15-20 \mathrm{~dB}$, depending on the location of the transmitter. It is noted that in areas, where radio propagation is dominated by Line of Sight $(\mathrm{LoS})$ components (e.g. along the corridor), the application of Frequency Selective Surfaces on the external walls had a minimal effect.

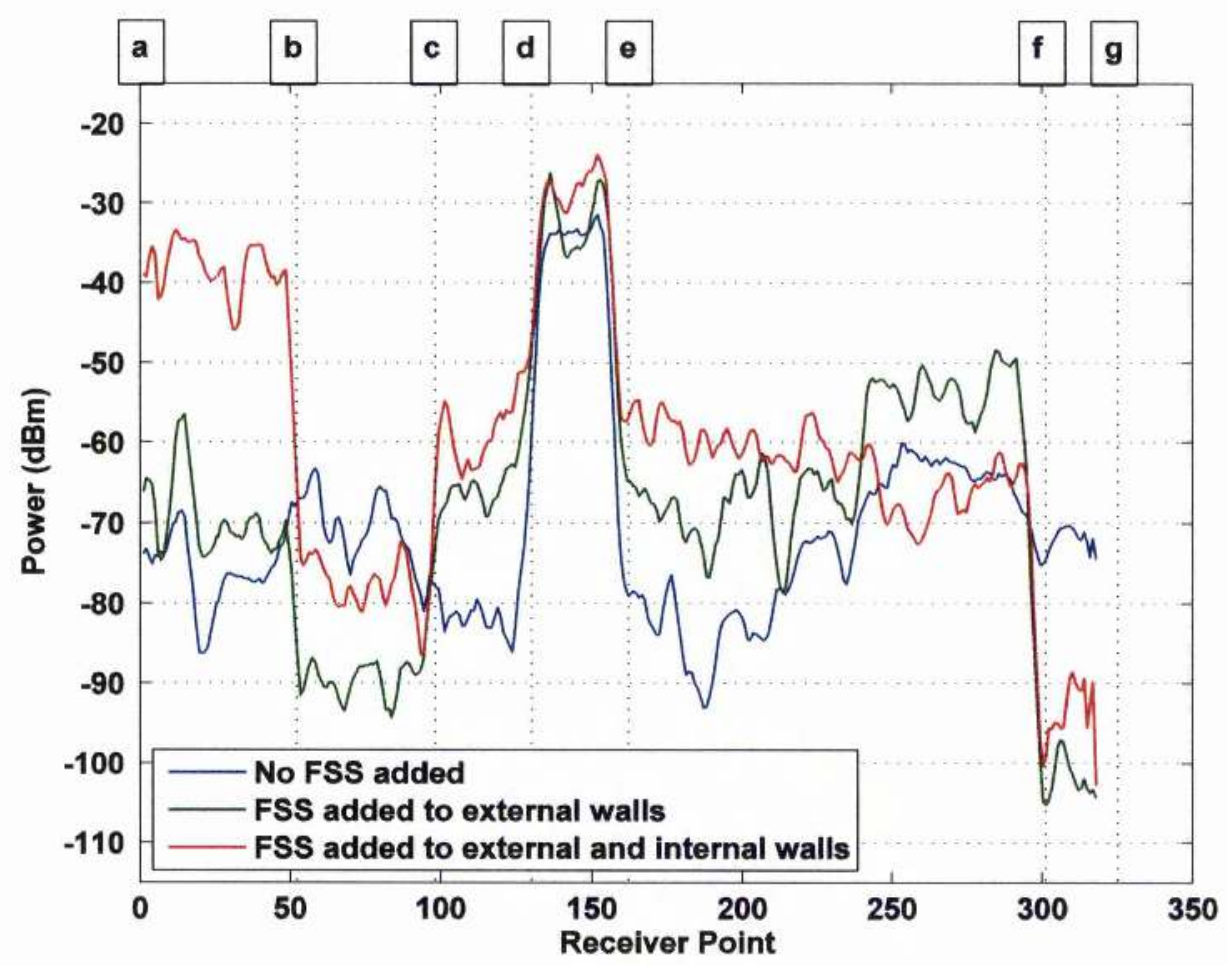

Figure 7-10: Comparison of different cases at $2.4 \mathrm{GHz}$ for scenario 2 . The letters at the top of the

figure correspond to the points along the estimation route shown in Figure 7-9

Results also suggest the received field inside the meeting room has not been significantly increased. Therefore, the next step into this investigation was to find a way to channel the signal along the corridor and increase the coverage in this area. As already observed from the previous simulation results, the easiest way to achieve this is to deploy FSSs on the internal walls (corridor walls) as well. Results presented in Figure 7-10 (red line) suggest that there is a significant increase of field strength in the meeting room compared to the non-FSS case (blue line). It is also 
noted that the field strength inside office rooms 3,4 and 5 has not decreased since the attenuation suffered by the rays while crossing the internal walls of these rooms is compensated by the stronger multiple reflections along the narrow corridor. However, this kind of compensation does not influence the received power outside the building, for the case where FSS are added only to the external walls. In this case the ray paths which undergo multiple reflections on the external walls are bigger in length, compared to the paths which undergo multiple reflections along the narrow corridor. This effectively means that these components will suffer higher path loss and they will not contribute significantly to compensate the power reduction due to the transmission through the wall. Similarly to scenario 1, results suggest that in certain scenarios, FSS will act as passive repeaters, channelling the signal to areas of interest while at the same time restricting coverage to other areas.

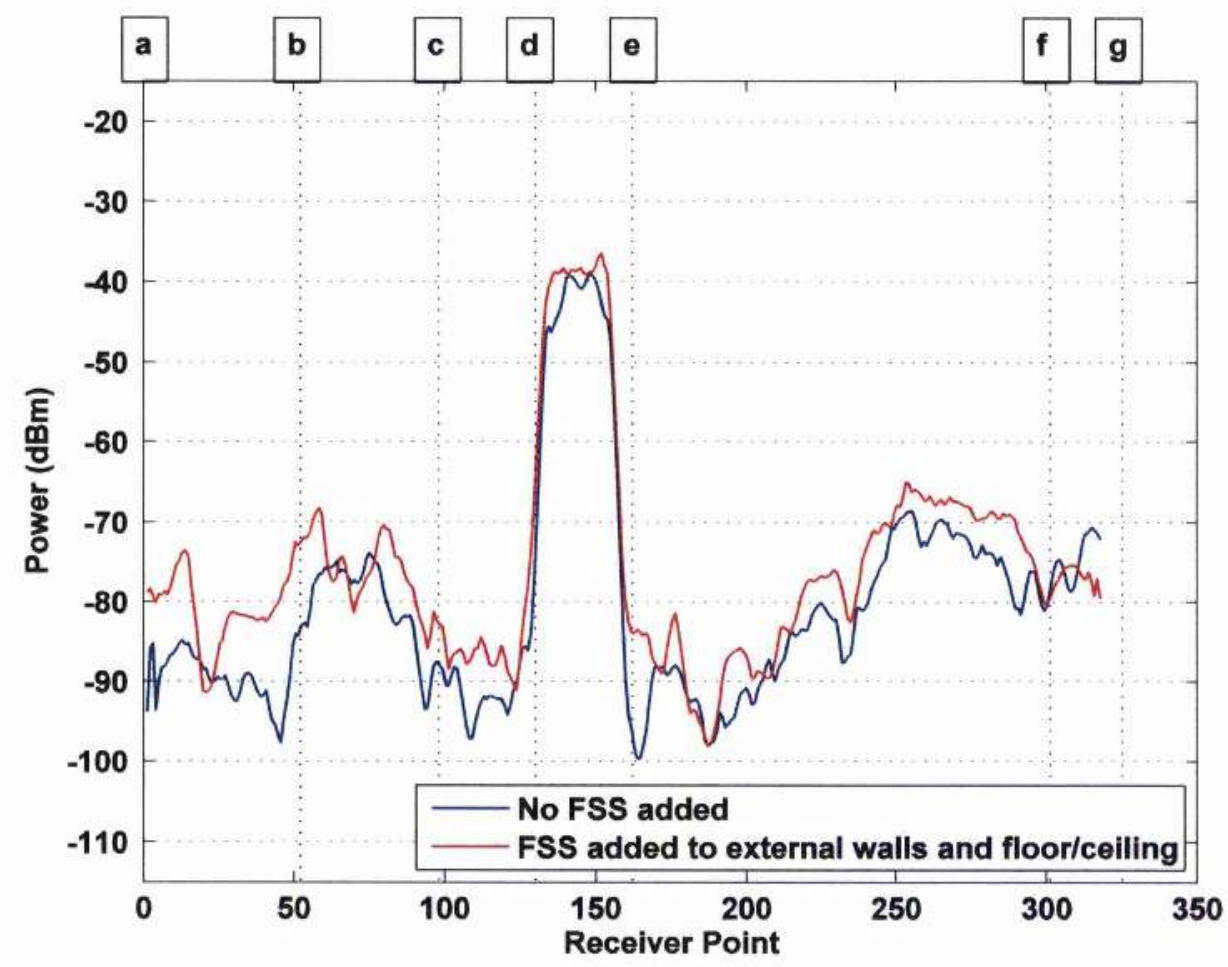

Figure 7-11: The Effect of a 2.4GHz tuned FSS on a 5.2GHz wireless system (scenario 2)

Figure 7-11 demonstrates the frequency selectivity of the deployment. It is again observed that the deployed FSSs do not significantly affect the radio propagation characteristics of any other system transmitting on a different frequency $(5.2 \mathrm{GHz}$ in this case). 


\subsection{Scenario 3: Outdoor to Indoor-Operation of various wireless systemis}

The scenarios investigated so far have dealt with indoor wireless environments where a WLAN system operating at $2.4 \mathrm{GHz}$ was in operation. In order to study the basic isolation between various networks operating in the same area, the outdoor/indoor scenario shown in Figure 7-12 was simulated. It consists of four buildings, each of which has a different wireless system in operation except houses 1 and 3 that have the same WLAN $(802.11 \mathrm{~b} / \mathrm{g})$ system transmitting on the same channel ( $\mathrm{Tx} 1$ and $\mathrm{Tx} 3$ ). House 2 has an $802.11 \mathrm{a}$ system transmitting at $5.2 \mathrm{GHz}(\mathrm{Tx} 2)$. Also a GSM Base station (Tx4 at $900 \mathrm{MHz}$ ) is located in the area. The systems installed in Houses 1 and 2, transmit sensitive data, and therefore to further improve security, the spillover of radio waves outside these buildings needs to be decreased. For this reason FSS have been added to the external walls of these two buildings, tuned at $2.4 \mathrm{GHz}$ and $5.2 \mathrm{GHz}$ respectively.

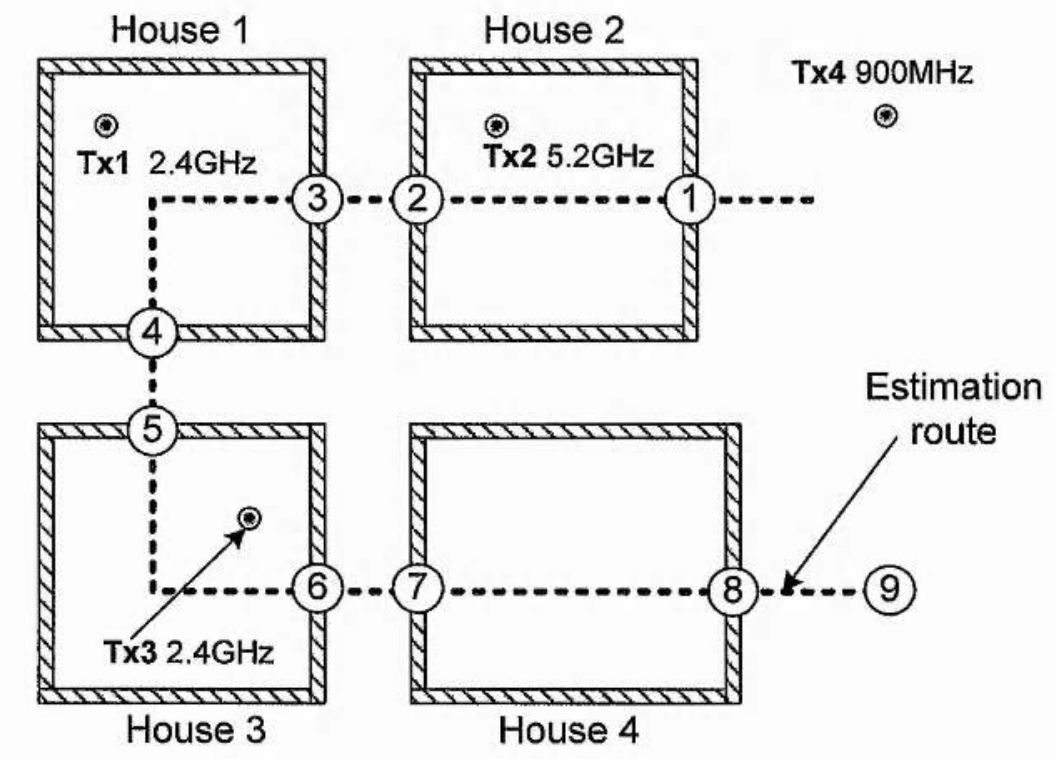

Figure 7-12: Outdoor to Indoor and Indoor to Outdoor Scenario

Figure 7-13 and Figure 7-14 present the potential benefit when FSSs are added to the external walls of Houses 1 and 2 . It is observed that the respective transmission for each system is blocked from escaping the wanted area, effectively increasing wireless security. The FSS added to the external walls of House 1 serves also another purpose. Suppose that Tx3 in House 3 is transmitting on the same channel as Tx1 and this building does not have FSS deployed on its walls. The presence of FSS on the walls of House 1 significantly reduces the interference to this system as demonstrated in Figure 7-15. 
In addition, the deployment of FSS on any of the buildings, should not affect the operation of any commonly used wireless networks such as the GSM system. Figure 7-16 shows that the deployed FSSs have a minor effect on the $900 \mathrm{MHz}$ transmission of the local GSM Base station.

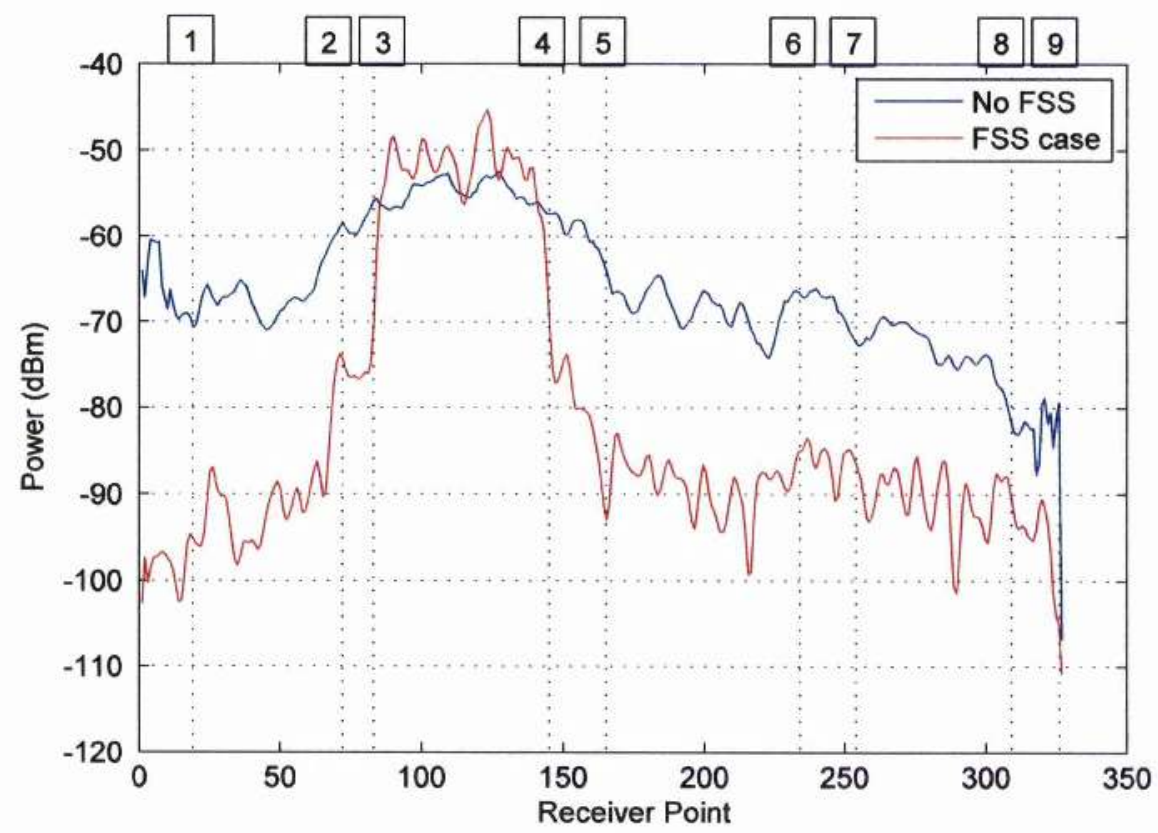

Figure 7-13: Tx1 transmitting at $2.4 \mathrm{GHz}$

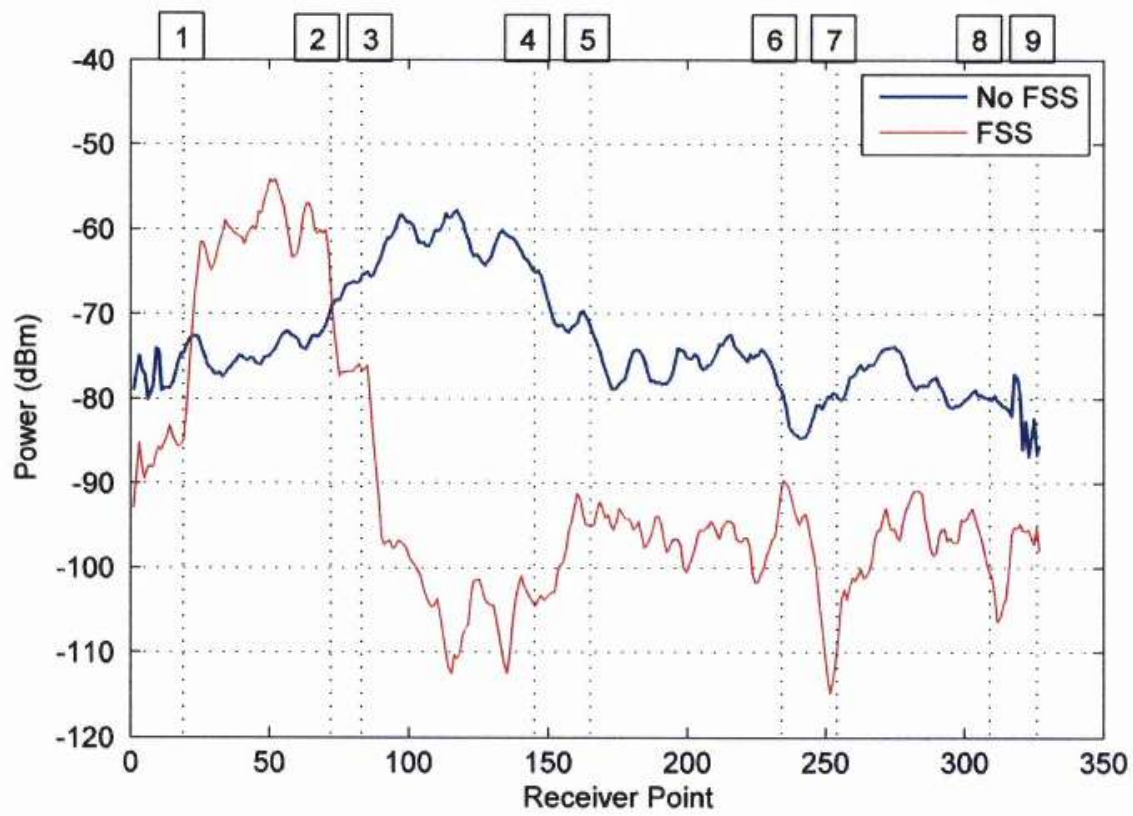

Figure 7-14: Tx2 Transmitting at 5.2GHz 


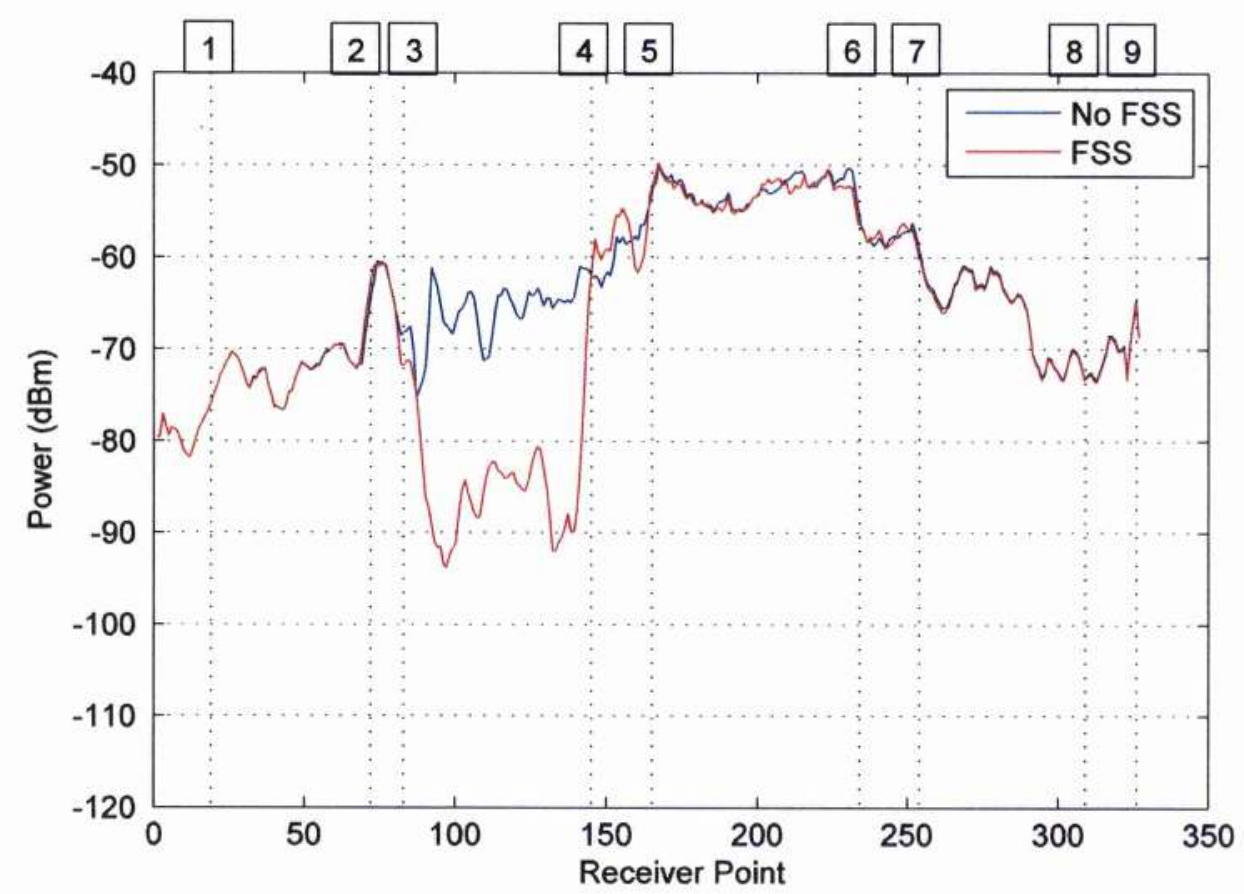

Figure 7-15: Tx3 Transmitting at $2.4 \mathrm{GHz}$

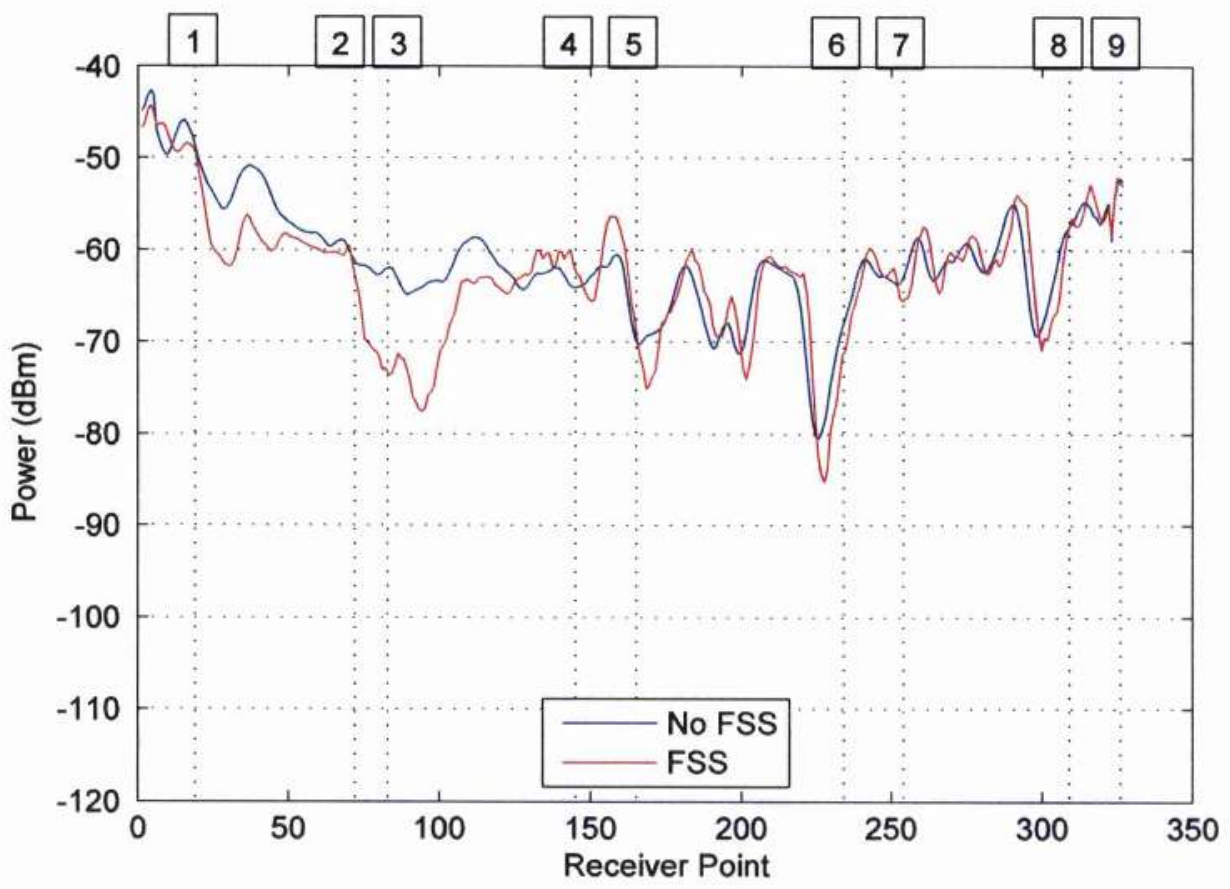

Figure 7-16: Cellular Base Station Transmitting at $900 \mathrm{MHz}$ 


\subsection{MIMO in FSS Environments}

Multiple Input/Multiple Output (MIMO) systems, initially introduced by Winters [104] consist of an array of transmitting and an array of receiving antenna elements as shown in Figure 7-17. Such systems have the potential to achieve high capacities as well as diversity gain depending on the propagation environment [106]. The increasing capacity of a MIMO system relies strongly on the richness of multipath rays which provide uncorrelated or low-correlated channels [107]. This means that the MIMO capacity increases as correlation decreases. It has been proven theoretically that the capacity of a MIMO channel also increases linearly with the number of transmitting and/or receiving antenna elements [105][108]. This is due to the decomposition of the channel into an equivalent set of spatial sub-channels [109].
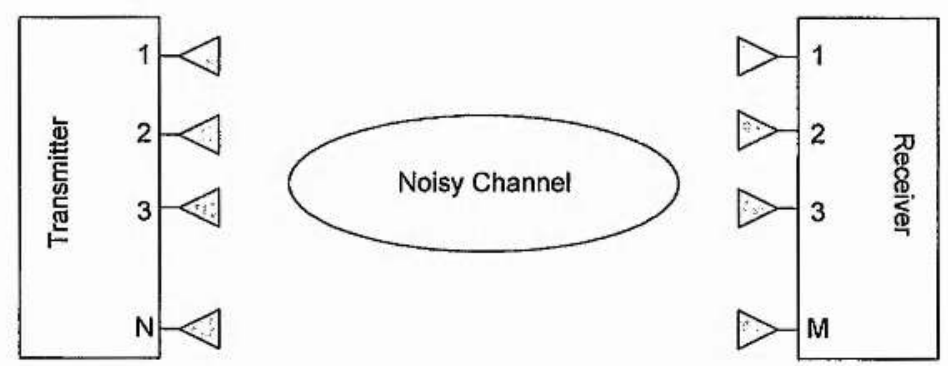

Figure 7-17: MIMO ChanneI

Literature includes some work on the applicability of MIMO systems for indoor wireless environments. The authors of [106] have performed measurements of a MIMO system under strong and weak Line of Site (LOS) conditions. They verified that the capacity decreases as the distance from the transmitting array increases because the transmission correlation increases. In [107] and [111] it is shown that the performance of a MIMO system is influenced by the arrangement of the array antenna elements as well as the indoor propagation environment. The authors of [110] have studied the influence of homogeneous and complex building structures on the capacity of indoor MIMO systems. They have shown through FDTD simulations that the complex structure can produce lower correlation and effectively higher MIMO capacities.

For the conventional Single Input Single Output (SISO) systems studied so far the fundamental formulation of capacity proposed by Shannon is given by,

$$
C=\log _{2}\left(1+\frac{S}{N}\right)
$$

where $C$ is the capacity in bps/Hz, $S$ is the signal power in watts and $N$ is the noise power in watts. For the $N \times M$ MIMO system shown in Figure 7-17, channel capacity is calculated using [112]:

$$
C=\log _{2}\left[\operatorname{det}\left(I+(\rho / N) H H^{T}\right)\right]
$$


Where $\rho$ is the average Signal to Noise Ratio (SNR) over all the MIMO branches, $I$ is the $N \times N$ identity matrix and $H$ is the channel matrix with $H^{T}$ being its conjugate-transpose matrix.

Since the presence of FSS in an indoor environment can increase the presence of strong reflected components in every receiving location it would be interesting to investigate any potential benefits in terms of capacity and diversity gain from using MIMO systems in an FSS indoor environment. The idea is that the presence of various multipath components at every receiving element would make the channel more Rayleigh (higher K-factor) achieving lower correlation and hence higher capacity. It is assumed that the conventional SISO system operating in the indoor scenario presented in section 7.3 is replaced by a $2 \times 2$ MIMO system. Both the Transmitter and Receiver is assumed to consist of two isotropic antenna elements, transmitting $0 \mathrm{dBm}$ and spaced by $\lambda / 2(\lambda=12.5 \mathrm{~cm})$. Simulations have been carried out along the estimation route depicted in Figure 7-9. In order to be able to capture multipath fading effects the spacing between the individual receiver locations was set to $\lambda / 10$.

Figure 7-18 shows the capacity complementary cumulative distribution function (CCDF) plots for various cases under investigation; the non-FSS case, the case where FSS is added on the external walls and the case where FSS is added on the internal and external walls. The results verify that the presence of Frequency Selective Surfaces in various positions inside the building can achieve higher capacities due to the creation of low correlated channel. These results are also compared with a conventional SISO system. Figure 7-19 shows the CCDF of the capacity multiplier (MIMO Capacity/SISO Capacity).

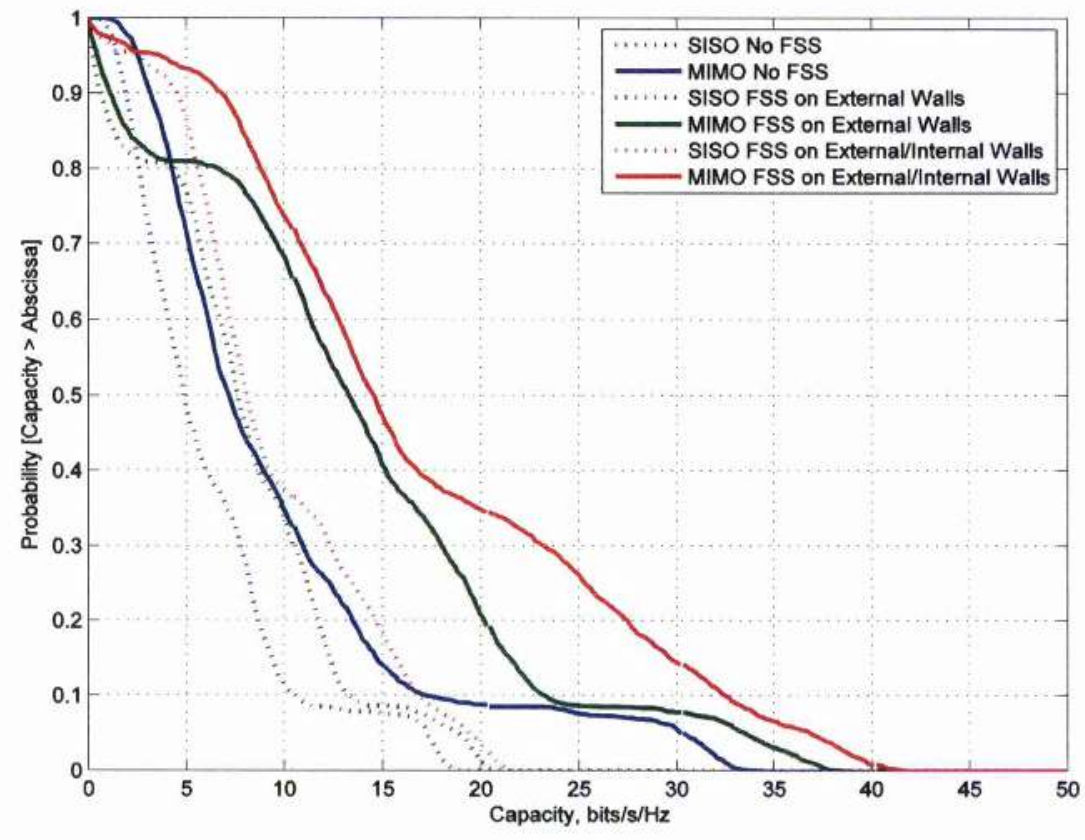

Figure 7-18: Capacity CCDFs for the various cases 


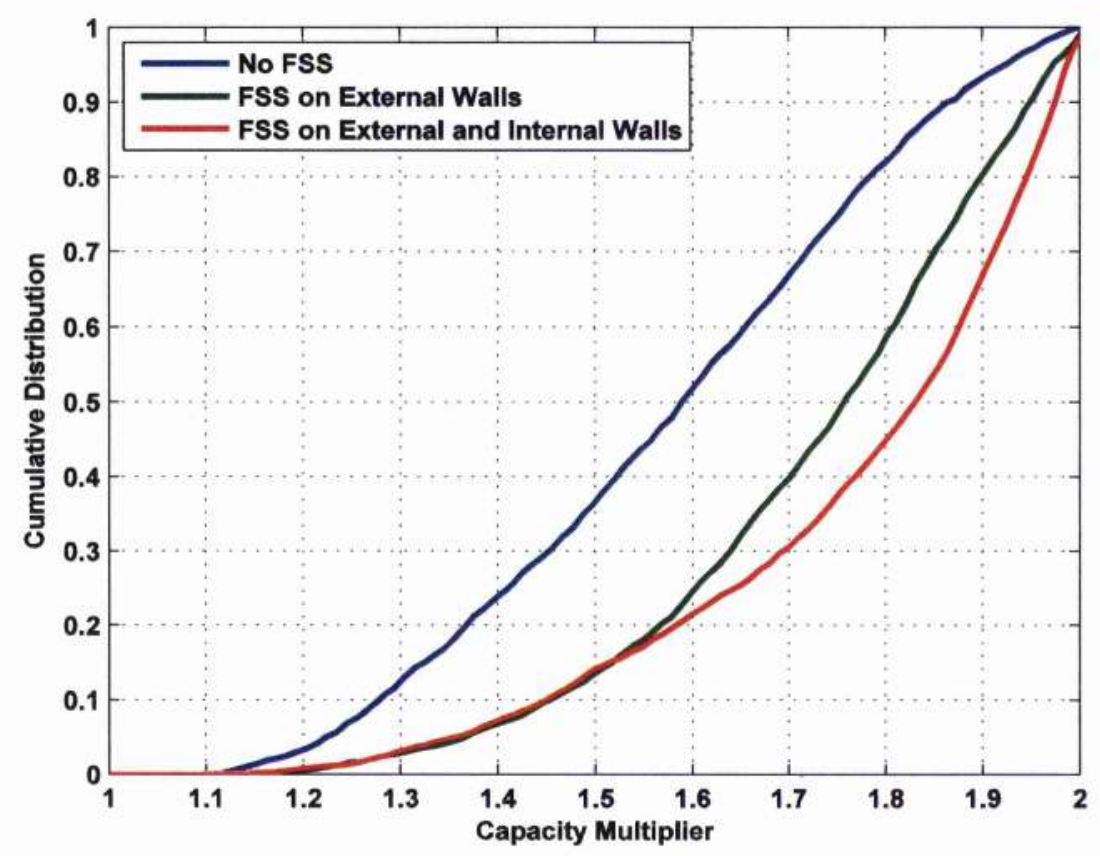

Figure 7-19: Capacity Multiplier CCDFs for the various cases

\subsection{Summary}

The basic isolation and passive amplification capabilities of FSS were demonstrated through the use of a specially modified ray tracing algorithm that accounts for the FSS deployment on the buildings' interfaces as presented in chapter 6 . Based on this model, various indoor and outdoor to indoor wireless environments where simulated, highlighting typical isolation and amplification figures that can be obtained. These figures will depend on the specific type and setup of the FSS used. Results suggest that FSS deployment can be used in wireless environments in order to selectively increase or restrict coverage for specific wireless system transmissions without affecting the operation of other wireless networks operating on different frequencies. Increasing signal strength would enable the reception of services that require higher data rates such as video streaming providing a more reliable and stable solution, whereas restricting coverage outside the wireless network operational area, minimises the possibility that signals can be picked up by unauthorised users which effectively could increase wireless security. A potential signal increase would effectively mean higher Signal to Noise Ratio (SNR) which is turn, for a WLAN system, would mean higher data rate (Mbps) and lower packet error rate (PER). In order to quantify the potential increase in PER performance, given an increase in signal 
strength (SNR) various other factors such as transmission rate, modulation and coding scheme, fading characteristics, noise etc. The authors of [113] have quantified this increase by plotting the PER versus SNR for various channel conditions (Ricean k-factors, different transmit rates and modulation schemes) in OFDM systems where Space Time Block Codes (STBC) are used. Their investigation dealt with both SISO and MIMO systems. Similar work has been carried out in [114] where a Viterbi decoder is used for the OFDM system. Also, for systems operating on the same or close enough frequency channels, interference between them can be significantly reduced providing higher reliability. Proper FSS deployment can also assist signal channelling or confine coverage in specific areas that are difficult to access with a conventional setup of a single transmitter. This can be used as an alternative solution to the conventional methods proposed over the years for improving wireless coverage, having its own advantages. Conventional methods include leaky feeders, active and passive repeaters, distributed antennas, etc [115]-[119]. Distributed transmitters (access points) or active repeaters which both require power to operate with the latter one requiring good isolation between the receiving and re-transmitting antennas, otherwise the system might become oscillatory. Also with active repeaters, the received noise and interference is reradiated on both the forward and reverse link [120].

An initial investigation was carried out demonstrating that the usage of FSS can increase capacity in MIMO systems. This is based on the presence of strong multipath components which give rise to a low correlated Rayleigh Channel. 


\section{Chapter 8}

\section{Conclusion and Future Work}

\subsection{Conclusion}

It is clear that the first hop between the user and the backbone network of almost all communication systems is turning wireless. Unlike conventional wired systems where the information follows a predetermined path along a wired grid, in wireless communication, information is passed through multiple routes by the propagation of radio waves in space. Radio wave propagation through this invisible channel is more difficult to define and is governed by complex parameters and propagation mechanisms. In order to support the need of high data rates and without ignoring the secure passing of information, such systems need to be carefully designed and deployed.

This research work started with a literature survey, which covered the basic principles of electromagnetic wave propagation relevant to path loss prediction in wireless communications, highlighting all its relevant propagation mechanisms and parameters. These mechanisms, namely reflection, refraction, diffraction and scattering, together with basic antenna and environment parameters (electrical or constitutive parameters) are of great importance in order to implement a propagation model that can be used to predict radio propagation for a wireless system. Radio propagation prediction has become a useful tool in the hands of a wireless system designer for characterising the wireless channel and evaluating the system to be deployed prior to the actual deployment. For this reason, this work has looked into various issues related to radio propagation modelling emphasising on deterministic Ray Tracing techniques and highlighting the major limitations and factors that may give rise to errors.

The main part of this research started with an investigation of radio propagation through typical building interfaces. When these interfaces are assumed to be made of homogeneous 
materials, the interaction of radio waves with them can be easily defined and predicted. However, this is not always the case, as buildings' interfaces are also non-homogeneous. More specifically, they usually present some periodic variations that give rise to reflections in non-specular directions. The web and void design of the individual blocks and their arrangement within a building wall/interface creates a periodic structure, which exhibits frequency dependent transmission and reflection characteristics. This natural building behavior of typical periodic structures, such as masonry block and reinforced concrete walls has been examined through the use of a custom-written RCWA model. The developed model can model the reflection, transmission and scattering characteristics of structures that exhibit an internal periodic behaviour along one single axis (either $x$ - or $y$-axis). For this reason, the technique used for this work is referred to as ID-RCWA. For this method to apply, the internal structure of any periodic wall should be consistent with the RCWA structure presented in this thesis (Figure 4-1). Therefore, for a typical masonry block wall, some minor features of these blocks (such as the thin rectangular slot in the middle and the cutout in one side) have been neglected. Also, for reinforced concrete walls periodicity has been assumed only in one direction ( $x$-axis), effectively modelling the behaviour of a reinforced concrete wall with vertical steel bars only. Another assumption was that the steel bars' circumference has been approximated with square having each side equal to the diameter of the steel bar. It has been observed that such structures exhibit resonances in their transmission and reflection characteristics, which seem to depend on the angle of incidence, the periodicity of the structure, the wall thickness and the constitutive parameters of the walls' materials. They also give rise to the propagation of various higher order modes (harmonics) in various angles of departure, which sometimes carry more energy than the specular ones. This scattering behaviour needs to be modelled and incorporated into a deterministic Ray-Tracing model if highly accurate results are required. However, this behaviour was beyond the scope of this research and hence only an initial flavour of it has been given in Chapter 4 .

The frequency selectivity exhibited by building structures has raised the idea of transforming buildings into frequency selective ones, where signals of specific frequencies are not allowed to enter and/or escape the building. Such a transformation would enable the frequency selective "tuning"/improvement of the propagation characteristics of the wireless communication systems operating in the building without affecting the operation of other systems in the area transmitting on different frequencies. However, since the internal structure and parameters of building periodic interfaces are usually unknown, it would be more or less impossible to utilise this natural frequency selectivity until an easy way will be found to " $\mathrm{x}$-ray" a building. A way to easily transform building interfaces into frequency selective, tuneable at a desired frequency range, is to deploy surfaces on those interfaces which have frequency selective characteristics. These surfaces are called frequency selective surfaces whose principles and deployment on 
building walls are studied in Chapter 5. Practical considerations regarding the deployment of FSS in wireless environments, such as the angle of incidence, and polarisation of the incident wave have been demonstrated. Extensive investigation has been carried out through both CFDTD simulations and anechoic chamber measurements, to study the effect of deploying frequency selective surfaces on conventional building materials. It was observed that once the FSS are attached on building walls detuning effects take place, which depend on the electrical parameters and dimensions of the supporting walls. For the proposed FSS application in wireless environments, it would be difficult, if not impossible, to precisely know the wall characteristics. In addition, it is usually the case in indoor and outdoor environments that walls have different morphological and geometrical properties. This would necessitate fabrication of various FSS designs so as the overall structure (FSS-wall) be tuneable to the desired frequency considering also the different effects of the different supporting walls. Therefore the investigation was concentrated on finding a "break-point" air-gap, beyond which the FSS response is not affected by the presence of any building wall. It was observed that for air-gaps greater than $\lambda / 10$, the FSS standalone tuning frequency is not affected by any homogeneous or periodic structure that exists behind it [94]. This air-gap appears to be dependent only on the wavelength of transmission and is not affected by the FSS design and the morphological/geometrical properties of the building walls. This would effectively give the flexibility to the designer to fabricate a single FSS design and safely deploy it on any building wall without worrying about any possible detuning effects.

To study the effect of FSS when these are deployed in wireless communication environments, a Ray Tracing model has been developed and modified to incorporate the FSS frequency characteristics. This model was developed in MATLAB, utilising the image method. It considers up to a user-defined number of reflections and an unlimited number of refractions (transmissions through). The limitation of the model is that it considers only one single UTD diffraction and that this diffraction (if it exists) happens only prior to any other propagation mechanism. Also, the model does not incorporate the periodic structure behaviour as it assumes specular reflection and transmission from homogeneous structures. Despite these assumptions, a comparison of the model predictions with measured results ( $2^{\text {nd }}$ floor of the CCSR) has indicated a reasonably good agreement. This Ray-Tracing model has been enhanced to incorporate the theoretical behaviour of FSS by means of pre-calculated CFDTD reflection and transmission coefficients. Although the frequency selectivity of FSS is incorporated, the model does not consider the FSS non-specular scattering. The FSS-Ray Tracing model was also validated through a small scale indoor FSS environment constructed and measured in a controlled environment (anechoic chamber).

Based on this model, various indoor and outdoor to indoor wireless environments where simulated, highlighting typical isolation and amplification figures that can be achieved. These 
figures will depend on the specific type and setup of the FSS used. The results suggest that proper FSS deployment can be used in such wireless environments in order to selectively increase or restrict coverage for specific wireless systems without affecting the operation of any other wireless networks that might transmit on other frequencies. Increasing signal strength would enable the reception of services that require higher data rates such as video streaming, providing a more reliable and stable solution by offering a lower packet error rate (PER) and higher trhoughput, whereas restricting coverage outside the wireless network operational area, minimises the possibility that signals can be picked up by non-authorised users effectively increasing wireless security. Also, for systems operating on the same or close enough frequency channels, interference between them can be significantly reduced providing higher reliability. Proper FSS deployment can also assist signal channelling or confine coverage in specific areas that are difficult to access with a conventional setup of a single transmitter. It has been also shown that when coverage isolation is required for specific regions of the environment, doors (and also windows) that can act as sources of spilled over rays into these regions should be covered with FSS and remain closed. An initial investigation was carried out demonstrating that the usage of FSS in an indoor environment can increase capacity in MIMO systems through the use of a $2 \times 2$ MIMO channel. This is due to the presence of stronger multipath components which give rise to low correlated channel.

Despite all the benefits presented in this thesis, from utilising frequency selective surfaces in wireless environments, someone can argue on the practical implications of this solution. One of the major practical implications is that the actual deployment of FSS on building walls is causing detuning effects. This work has proposed a way to overcome this problem by placing the FSS at a certain distance $(\lambda / 10)$ away from the building wall. Also someone can question the viability of this method by raising the question on what would happen if some period after the FSS deployment, the building tuning requirements change. This can be due to the change/upgrade of the wireless system operating in the building; for example a change from an $802.1 \mathrm{lb}(2.4 \mathrm{GHz})$ system to an $802.11 \mathrm{a} / \mathrm{g}(5.2 \mathrm{GHz})$ system. A possible solution to this could be the deployment of dual band FSS tunable at both frequencies, or even the deployment of active FSS (see section 5.1.2). In active FSS, the frequency properties can be varied by either using semiconductor devices (i.e. PIN diodes) placed into the elements or by printing the elements on substrates whose dielectric properties can be varied. 


\subsection{Future Work}

The work can be further extended in the following fields:

Advanced Hybrid Ray tracing model: Investigate the benefit from using a MIMO or SIMO wireless system by considering the natural periodic behaviour of building interfaces. This should include the development/use of a hybrid Ray Tracing model that incorporates the interfaces periodic behaviour by using the RCWA method. The current model used for the simulations in chapter 7 can cope only with homogeneous walls because it ignores the generation of nonspecular components. A hybrid RCWA-Ray Tracing can be used to provide more realistic predictions by considering the frequency selectivity and non-specular scattering arising from the internal periodic structure of building walls. RCWA needs to be enhanced to account for double periodic $(x-y)$ interfaces since the current model accounts wall periodicity only in one direction. Validate the results through anechoic chamber measurements and in-situ measurements using a MIMO sounder.

Optimum FSS deployment: Investigate whether more selective FSS deployment in various wireless environments can achieve similar or even better results than then ones presented in chapter 7. Deploying FSS in all the building walls can be in some cases non-practical and financially non-viable solution. It should be investigated whether placement/deployment of small FSS boards in selective positions in buildings can achieve similar or even better results. The process can be algorithmically automated to predict optimum positions in wireless environments that finite sized FSS boards can be deployed to achieve the required system specifications.

Periodic materials / FSS measurements: Perform anechoic chamber measurements to further study the effect of periodic walls/materials on the Frequency Selective Surfaces' behaviour, when the latter are deployed on such walls. In this work FSS where deployed only on homogeneous structures indicating that a safe air-gap distance (between the FSS and the supporting wall) exists, beyond which the FSS response is not affected. Initial simulation results have indicated that similar behaviour can be achieved using periodic supporting walls, but this needs to be further supported through measurements.

MIMO/FSS: This work has given an initial flavour of the effect of FSS on MIMO systems in indoor environments. Work can be further extended by investigating the capacity increase and diversity gain that can be achieved for various types of environments and antenna element combinations. 


\section{Appendix}

\section{Appendix 1: EMCO MODEL 3115 Double Ridge Guide Antenna}

The EMCO Model 3115 Double Ridge Guide Antenna is a linearly polarised broadband antenna covering the frequency range of $1 \mathrm{GHz}$ to $18 \mathrm{GHz}$.

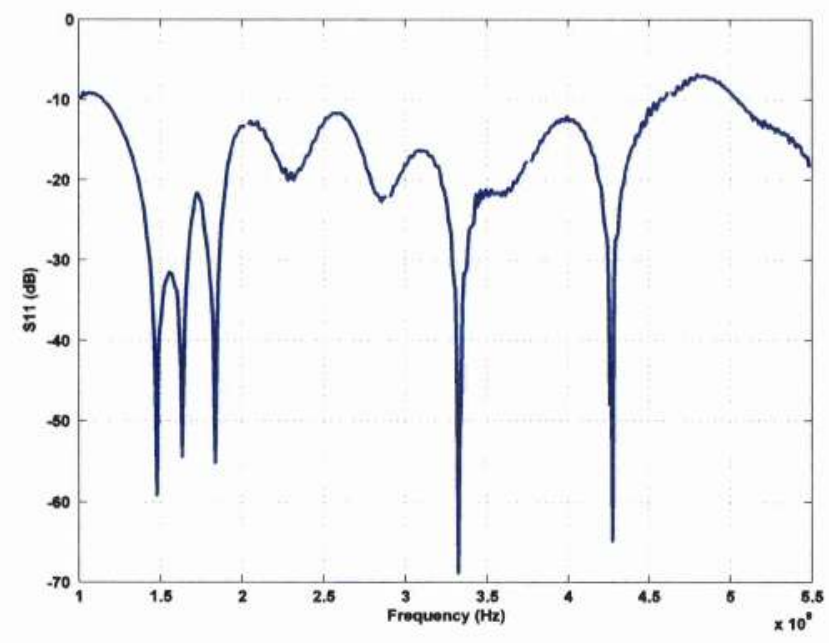

Measured Antenna S11

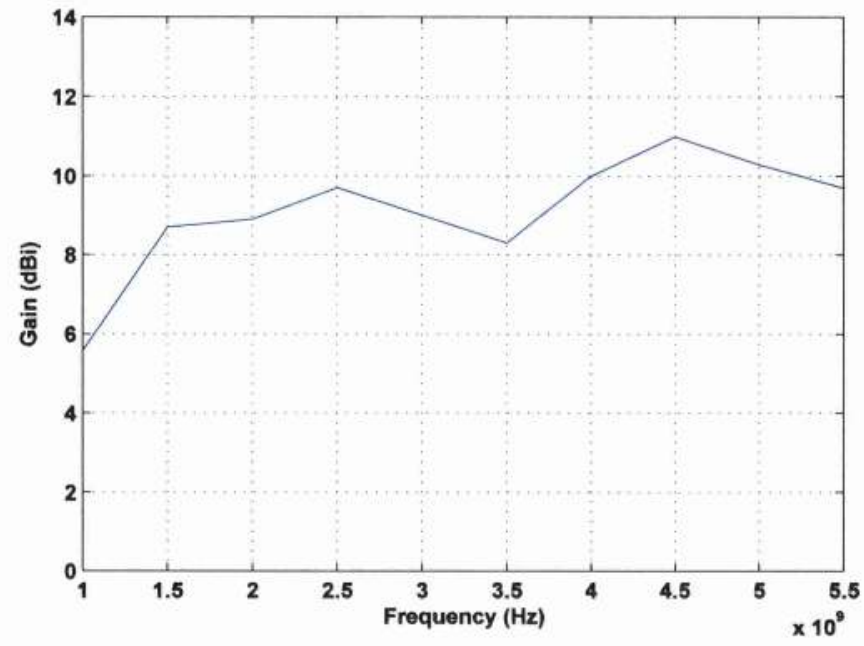

Antenna Gain in dBi 


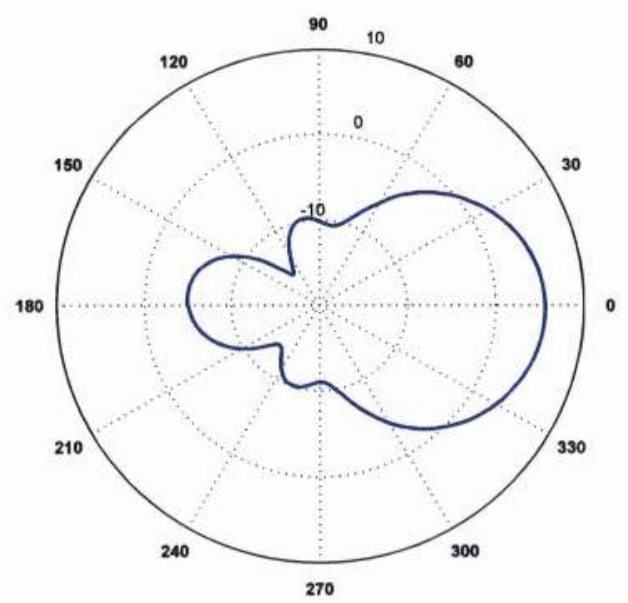

$1 \mathrm{GHz}$

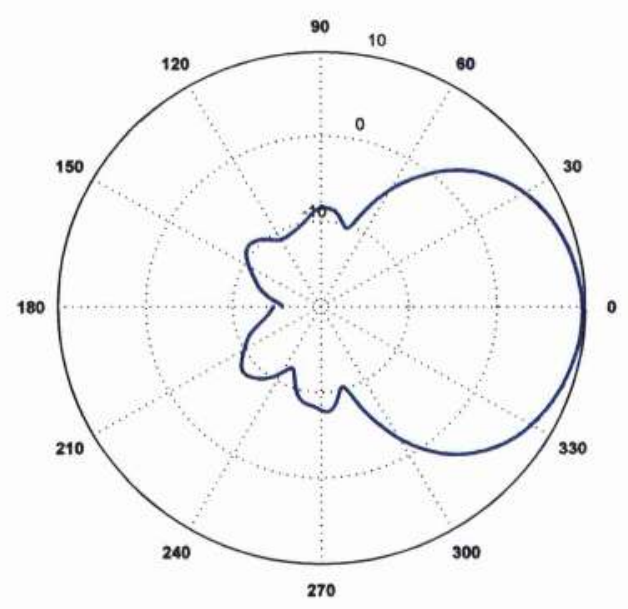

$2.4 \mathrm{GHz}$

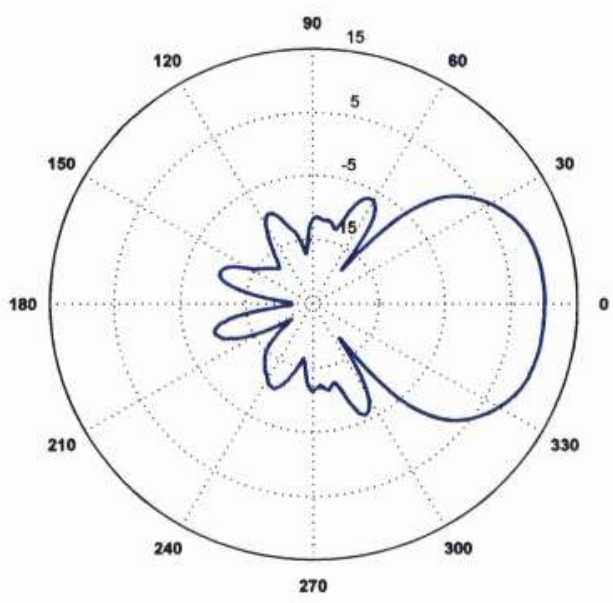

$4 \mathrm{GHz}$

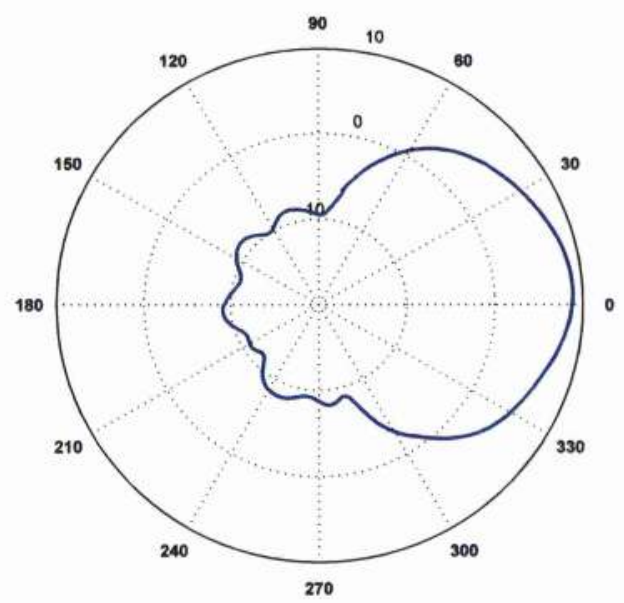

$2 \mathrm{GHz}$

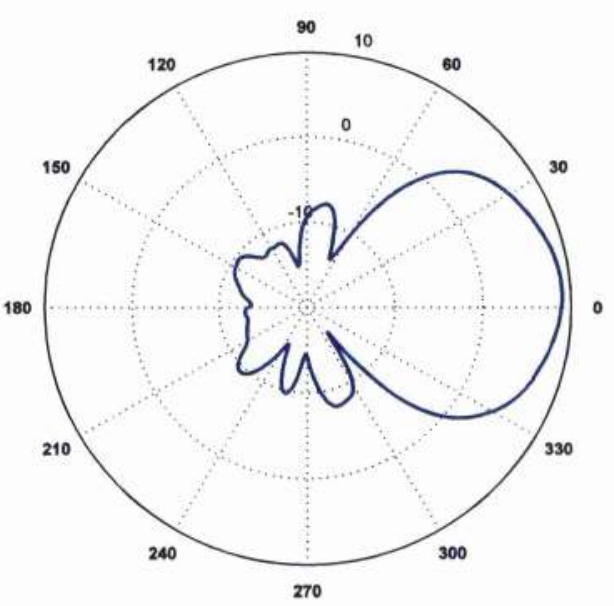

$3 \mathrm{GHz}$

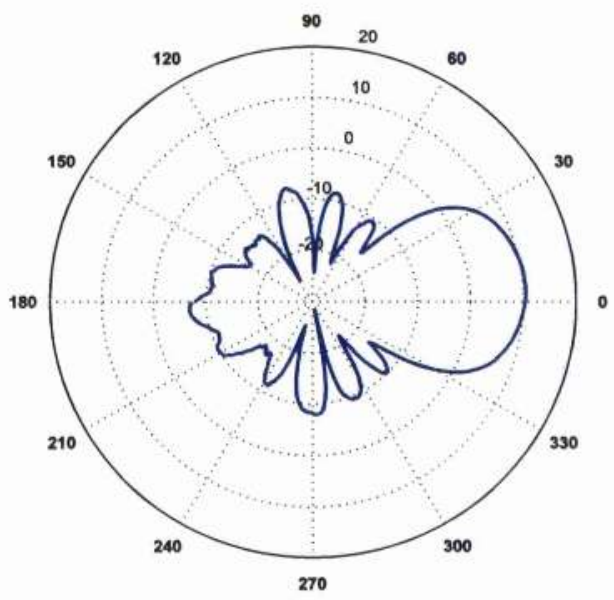

$5 \mathrm{GHz}$

E-Plane Measured Radiation Patterns 


\section{Appendix 2: MIMO/SISO Capacity Calculations}

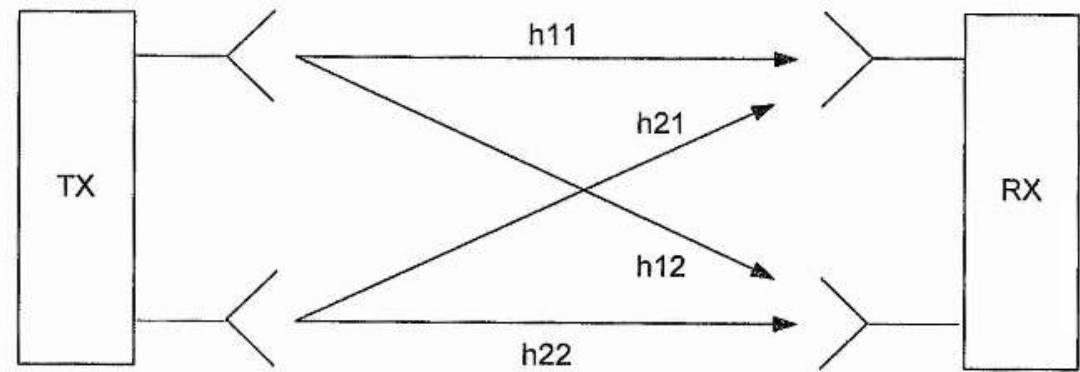

The normalised channel matrix $\mathrm{H}$ is calculated using:

$$
H=A *\left[\begin{array}{ll}
h_{11} & h_{12} \\
h_{21} & h_{22}
\end{array}\right]
$$

,where

$$
A=\sqrt{\left|h_{11}\right|^{2}+\left|h_{12}\right|^{2}+\left|h_{21}\right|^{2}+\left|h_{22}\right|^{2}}
$$

And the channel impulse response $h_{x y}$ for the channel between antenna elements $\mathrm{x}$ and $\mathrm{y}$ is given by:

$$
h_{x y}=10^{\text {Power }_{d B m} / 20 \exp \left(j \theta_{\text {rad }}\right)}
$$

The capacity of both a $2 \times 2 \mathrm{MIMO}$ and a SISO system are calculated from the instantaneous SNR and the Normalised channel matrix H. 100dB have been added to the path loss to calculate the SNR as:

$$
S N R=\frac{\left|h_{11}\right|^{2}+\left|h_{12}\right|^{2}+\left|h_{21}\right|^{2}+\left|h_{22}\right|^{2}}{4} * 10^{100 d B / 10}
$$

SISO Capacity: $C_{S I S O}=\log _{2}(1+S N R)$

MIMO Capacity: $C_{2 \times 2 \text { мIмо }}=\log _{2}\left[\operatorname{det}\left(I+S N R / 2 * H H^{T}\right)\right]$

I is an identity $2 \times 2$ matrix

$$
I=\left[\begin{array}{ll}
1 & 0 \\
0 & 1
\end{array}\right]
$$




\section{Bibliography}

[1] B. G. Evans and K. Baughan, "Visions of 4G", Electron. Commun. Eng. J., Vol. 12, pp. 293330, Dec 2000.

[2] C. A. Balanis, "Advanced Engineering Electromagnetics", John Wiley \& Sons Inc., ISBN: 0471-62194-3, New York 1989.

[3] A. Ishimaru, "Electromagnetic Wave Propagation, Radiation and Scattering", Prentice Hall Inc., ISBN: 0-13-249053-6, 1991.

[4] S. R. Saunders, "Antennas and Propagation for Wireless Communication Systems", John Wiley \& Sons Inc., ISBN: 0-471-98609-7, New York 1999.

[5] Katsuyoshi Sato, Hideki Kozima, Hiroshi Masuzawa, Takeshi Manebe, Toshio Ihara, Yoshinori Kasashima, Katsunori Yamaki, "Measurements of Reflection Characteristics and Refractive Indices of Interior Construction Materials in Millimeter-Wave Bands", $45^{\text {th }}$ IEEE Conf. Vehic. Techn., Vol.1, pp. 449-453, 1995

[6] W. D. Burnside, K. W. Burgener, "High Frequency Scattering by a Thin Lossless Dielectric Slab", IEEE Trans. Antennas Propag., Vol. 31, No. 1 pp. 104-110, Jan. 1983.

[7] A. Kedar, U. K. Reankar, "Parametric study of flat sandwich multilayer radome", Progress in Electromagnetics Research, PIER 66, pp. 253-265, 2006.

[8] D.J.Kozakoff, "Analysis of Radome - Enclosed Antennas", Artech House, Boston \& London, ISBN: $0890067163,1997$.

[9] R.E.Collin, Field Theory of Guilded Waves McGraw-Hill, 1960

[10] D. A. McNamara, C. W. I. Pistorius, J. A. G. Malherbe, "Introduction to the Uniform Geometrical Theory of Diffraction", Artech House Publishers, ISBN: 0-89006-301-X, 1990.

[11] Manuel F. Catedra, Jesus Perez-Arriaga, "Cell planning for Wireless Communications", Mobile Communications Series, Artech House Publishers, 1999.

[12] H.D.Hristov, Fresnel zones in Wireless Links, Zone Plate Lenses and Antennas Artech House, Boston. London, 2000.

[13] T.S.Rappaport, Wireless Communications - Principles \& Practice, $1^{\text {st }}$ edition Prentice Hall, 1996.

[14] W. K. Tam, V. N. Tran, "Propagation modelling for indoor wireless communication", Electronics \& Communication Engineering Journal, Vol. 7, Issue: 5, Oct. 1995 pp. 221 -228. 
[15] Y. Okumura, E. Ohmori, T. Kawano, K. Fukuda, "Field strength and its variability in VHF and UHF land mobile radio service", Rev. Electr. Commun. Lab. Vol. 16, 825-873, 1968.

[16] M. Hata, "Empirical Formula for propagation loss in land mobile radio services", IEEE Trans. Veh. Tech., Vol. 29, pp. 317-325, 1980.

[17] M. F. Ibrahim, J. D. Parsons, "Signal Strength prediction in built-up areas", Proc. IEE, Vol 130F, Issue 5, pp. 377-385, 1983.

[18] K. Allsebrook, J. D. Parsons, "Mobile radio propagation in British cities at frequencies in the VHF and UHF Bands", IEEE Trans. Veh. Tech. Vol. 26, No. 4, pp. 95-102, 1977.

[19] F. Ikegami, T. Takeuchi, S. Yoshida, "Theoretical prediction of mean field strength for urban mobile radio", IEEE Trans. Antennas Propag., Vol. 39, No. 3, pp. 299-302, 1991.

[20] J. Walfisch, H. L. Bertoni, "A theoretical model for UHF propagation in urban environments", IEEE Trans. Antennas Propag., Vol. 36, No. 12, pp. 1788-1796, 1988.

[21] COST 231 Final Report, "Digital mobile Radio: COST231 View on the Evolution Towards $3^{\text {rd }}$ generation Systems", Commission of the European Communities and COST Telecomunications, Brussels, 1999.

[22] W. C. Y. Lee, "Mobile Communications Engineering", McGraw Hill New York, ISBN: 0-471-57446-5, 1993.

[23] ITU, "Propagation data and prediction methods for the planning of indoor adiocommunication systems and radio local area networks in the frequency range $900 \mathrm{MHz}$ to 100 GHz", ITU-R P1238-2, 2007

[24] Georgia E. Athanasiadou, Andrew R. Nix, "A Novel 3-D Indoor Ray-Tracing Propagation Model: The Path Generator and Evaluation of Narrow-Band and Wide-Band Predictions", IEEE Trans. Veh. Tech., Vol. 49, No.4, July 2000.

[25] J.B.Keller, "Geometric theory of diffraction," Journal Optical Society of America, vol. 52, pp. 116-130, Feb.1962.

[26] R.G.Kouyoumjan and P.H.Pathak, "A uniform geometrical theory of diffraction for an edge in a perfectly conducting surface," Proceedings of IEEE, vol. 42, pp. 1148-1461, Nov. 1974.

[27] C. Tzaras, S. R. Saunders, "An Improved Heuristic UTD Solution for Multiple-Edge Transition Zone Diffraction", IEEE Trans. Antennas and Propag., Vol. 49, No. 12, pp. 16781682, Dec. 2001.

[28] R. J. Leubbers, "Finite conductivity uniform GTD versus knife edge diffraction in prediction of propagation path loss", IEEE Trans. Antennas and Propag., Vol AP-32, pp. 7076, Jan. 1984.

[29] R. J. Leubbers, "A heuristic UTD slope diffraction coefficient for rough lossy wedges" IEEE Trans. Antennas and Propag., Vol. 37, pp. 206-211, Feb. 1989. 
[30] J. F. Rouviere, N. Douchin, P. F. Combes, "Diffraction by Lossy Dielectric Wedges Using Both Heuristic UTD Formulations and FDTD", IEEE Trans. Antennas and Propag., Vol. 47, No 11, pp. 1702-1708, Nov. 1989.

[31] Schaubach, K. R., Davis N. J., "Microcellular Radio-Channel Propagation Prediction", IEEE Antennas and Propag. Mag., Vol. 36, No. 4, pp. 25-34 Aug. 1994.

[32] Magdy F. Iskander, Zhengqing Yun, "Propagation Prediction Models for Wireless Communication Systems", IEEE Transactions on Microwave Theory and Techniques, Vol. 50, No. 3, March 2002.

[33] C. W. Trueman, R. Paknys, J.Zhao, D. Davis, B. Segal, "Ray Tracing Algorithm for Indoor Propagation", Proc 16th Annual Review of Progress in Applied Computational Electromagnetics, Monterey, CA., pp. 493-500, March 2000.

[34] Marc Kimpe, Harry Leib, Olivier Maquelin, Ted H. Szymanski, "Fast computational Techniques for indoor radio channel estimation", IEEE Computational Science and Engineering, Vol. 1, Issue 1, January-February 1999.

[35] K. A. Remley, H. R Anderson, A. Weisshar, "Improving the Accuracy of Ray-Tracing Techniques for Indoor Propagation Modelling", IEEE Trans. Veh. Tech., Vol. 49, No. 6, pp. 2350-2358, Nov. 2000.

[36] Y. Wang, S. Safavi-Naeini, S. K. Chaudhuri, "A hybrid technique based on combining ray-tracing and FDTD methods for site-specific modelling of indoor radio wave propagation", IEEE Trans. Antennas Propag., Vol. 48, pp. 743-754, May 2000.

[37] R. J. Luebbers, "Finite conductivity uniform GTD versus knife edge diffraction in prediction of propagation path loss", IEEE Trans. Antennas Propag., Vol. AP-32, pp.70-76, Jan. 1984.

[38] R. J. Leubbers, "Comparison of lossy wedge diffraction coefficients with application to mixed path propagation loss prediction", IEEE Trans. Antennas Propag., Vol. 36, pp. 10311034, July 1988.

[39] G. E. Athanasiadou, A. R. Nix, "Investigation into the sensitivity of the power predictions of a microcellular ray-tracing propagation model", IEEE Trans. Antennas Propag., Vol. 49, pp. 1140-1151, July 2000.

[40] W. Honcharenko, H. L. Bertoni, "Transmission and Reflection Characteristics at concrete block walls in the UHF bands proposed for future PCS", IEEE Trans. Antennas Propag., Vol. 42, No. 2, pp. 232-239, Feb. 1994.

[41] M. Y. W. Chia, "The effects of reinforced concrete walls/floors on wireless personal communications systems (PCS)", International Symposium Antennas and Propagation 1995, vol. 4, pp. 1956-1959, June 1995. 
[42] R. A. Dalke, C. L. Holloway, P. McKenna, M. Johansson, A. S. Ali, "Effects of Reinforced concrete structures on RF communications", IEEE Trans. Electromang. Compatibility, Vol. 42, No. 4. Nov. 2000.

[43] C.L.Holloway, P.L.Perini, R.R.DeLyser, and K.C.Allen, "Analysis of composite walls and their effects on short-path propagation modeling," IEEE Transactions on Vehicular Technology, vol. 46, pp. 730-738, Aug.1997.

[44] S.V.Savov and M.H.A.J.Herben, "Modal transmission-line modeling of propagation of plane radiowaves through multilayer periodic building structures," IEEE Transactions on Antennas and Propagation, vol. 51, pp. 2244-2251, Sept.2003.

[45] M. Yang, S. Stavrou, "Three-dimensional modal transmission line method for radio wave propagation through periodic building structures", IEE Proc. Microwave Antennas Propag., vol. 152, No. 6, December 2005.

[46] M. Yang, S. Stavrou, "Rigorous Coupled Wave Analysis of Radio Wave Propagation Through Periodic Building Structures", IEEE Trans. Antennas Propag. Letters, Vol, 3, pp. 204-207, 2004.

[47] M. G. Moharam, T. K. Gaylord, "Rigorous Coupled-Wave analysis of planar grating diffraction", J. Opt. Soc. Am, Vol. 71, No. 7, pp. 811-818, July 1981.

[48] M. G.Moharam, D.A.Pommet, and E.B.Grann, "Stable implementation of the rigorous coupled-wave analysis for surface-relief gratings: enhanced transmittance matrix approach," Journal Optical Society of America A, vol. 12, pp. 1077-1086, May1995.

[49] M. G.Moharam, E.B.Grann, and D.A.Pommet, "Formulation for stable and efficient implementation of the rigorous coupled-wave analysis of binary gratings," Journal Optical Society of America A, vol. 12, pp. 1068-1076, May 1995.

[50] T. Tamir, S. Zhang, "Modal transmission-line theory of multilayered grating structures, "IEEE Journal of Lightwave Technology", Vol. 14, pp. 914-927, May 1996.

[51] S. Peer, J. T. Case, E. Gallaher, K. E. Kurtis, R. Zoughi, "Microwave Reflection and Dielectric Properties of Mortar Subjected to Compression Force and Cylindrically Exposed to Water and Sodium Chloride Solution", IEEE Trans. Instrumentation and Measurement, vol. 52, No. 1, pp. 111-118, Feb. 2003.

[52] S.Stavrou and S.R.Saunders, "Review of constitutive parameters of building materials," 12th International Conference on Antennas \& Propagation, vol. 2, pp. 211-215, 2003.

[53] M. G. Moharam, "Coupled-wave analysis of two-dimensional dielectric gratings", SPIE, Holographic Optics: Design and Applications, Vol. 883, pp. 8-11, 1988

[54] J. Jiang, "Rigorous Analysis and design of diffractive optical elements", $P h . D$. Thesis, Dept, Opt. Science, Univ. of Alabama, 2000.

[55] C.E. Shannon, "The zero-error capacity of a noisy channel", IRE Trans. Information Theory, Vol 2, Issue. 3, pp. 8-19, 1956 
[56] Ben A. Munk, "Frequency Selective Surfaces - Theory and design", John Wiley \& Sons Inc., ISBN: 0-471-37047-9, New York, 2000.

[57] D.S.Lockyer, J.C.Vardaxoglou and R.A.Simpkin, "Complementary Frequency Selective Surfaces", IEE Proc. Mircow. Antennas Propag., Vol. 147, Issue 6, Dec. 2000, pp. 501 507.

[58] T. K. Chang, R. J. Langley, E. A. Parker, "Active Frequency Selective Surfaces", IEE Proc. Mircow. Antennas Propag, Vol. 143, No. 1, Feb. 1996, pp. 62-66.

[59] R. J. Langley, E. A. Parker, "An equivalent circuit study of a PIN diode switched active FSS", Report to British Aerospace plc., Feb. 1990.

[60] N. V. Schuley, "Diode loaded Frequency Selective Surfaces", Proceeding of JINA92 International Conference on Antennas, Nice, France 1992, pp. 313-316.

[61] K. W. Dittrich, "Multifunctional Skins", Proceedings of $6^{\text {th }}$ Europ. Electromagnetic Structures Conference, Friedrichshafen Germany, 1991, pp. 1-13.

[62] T. K. Chang, R. J. Langley, E. A. Parker, "An Active Square Loop Frequency Selective Surface", IEEE Microwave and Guided Wave Letters, Vol. 3, No. 10, Oct. 1993.

[63] T. K. Chang, R. J. Langley, E. A. Parker, "Frequency Selective Surfaces on biased ferrite substrates", Electr. Letters, Vol. 30, No. 15, July 1994.

[64] A. C. de Lima, E. A. Parker, R. J. Langley, "Tunable Frequency Selective Surface using liquid substrates", Electr. Letters, Vol. 30, No. 4, Feb. 1994.

[65] P. Callaghan, E. A. Parker, R. J. Langley, "Influence of supporting dielectric layers on the transmission properties of frequency selective surfaces", IEE Proceedings, Vol. 138, No. 5, Oct. 1991.

[66] R. J. Luebbers, B. A. Munk, "Some effects of dielectric loading on periodic slot arrays", IEEE Trans. Antennas Propag., Vol. 26, No. 4, July 1978, pp. 536-542.

[67] R. Orta, R. Tascone, R. Zich, "Multiple dielectric loaded perforated screens as frequency selective surfaces", IEE Proceedings, Vol. 135, No. 2, April 1988, pp. 75-82.

[68] S. Contu, R. Tascone, "Scattering from passive arrays in plane stratified regions", Electromagnetics, Vol. 5, No. 4, 1985, pp.285-306.

[69] T. Cwik, R. Mittra, "The cascade connection of planar periodic surfaces and lossy dielectrics to form an arbitrary periodic screen", IEEE Trans. Antennas Propag., Vol. 35, No. 12, Dec. 1987, pp. 1397-1401.

[70] T. K. Wu. "Frequency Selective Surface and grid array", John Wiley \& Sons Inc., 1995. 
[71] H. Gniss, E. Zocher, "A Multibeam Cassegrain Antenna", $5^{\text {th }}$ Europ. Microw. Conf., 1975, pp. 466-470, Oct. 1975.

[72] V. D. Agrawal, W. A. Imbriale, "Design of a dichroic Cassegrain Subreflector", IEEE Trans. Antennas. Propag., Vol.27, No. 4, pp. 466-473, 1979.

[73] L. Young, L. A. Robinson, F. A. Pelow, "Meander line polarizer", IEEE Trans. Antennas Propag., Vol. 21, pp. 376-378, 1973.

[74] D. A. McNamara, D. E. Baker, "Design and performance of etched polirazation transformers for microwave frequencies", Proc. SAIEE Symposium Antennas Propag., pp. 19, 1983.

[75] K. Karkkainen, M. Stuchly, "Frequency Selective Surface as a polarisation transformer", IEE Proc. Microw. Antennas Propag., Vol. 149, No. 516, Dec. 2002.

[76] A. Tennant, B. Chambers, "A single-layer tuneable microwave absorber using active FSS", IEEE Microw. Wireless Components Letters, Vol. 14, No. 1, pp. 46-47, Jan. 2004.

[77] R. Mittra, C. H. Chan, T. Cwik, "Techniques for analysing Frequency Selective Surfaces -A Review”, IEEE Proc., Vol. 76, No 12, Dec. 1988.

[78] L. W. Henderson, "Introduction to PMM", Tech. Report 725582-5, Ohio State Univ. ElectroScience Lab., Dept of Electrical Eng., prepared under contruct No. F33615-83-C-1013 for the Air Force Avionics Laboratory, Air Force Wright Aeronautical Laboratories, Air Force Systems Command, Wright-Patterson Air Force Base, OH 45433, Feb. 1986.

[79] J. L. Volakis, A. Chatterjee, L. C. Kempel, "Finite Element Method for electromagnetics", IEEE Press, ISBN: 0-7803-3425-6, New York, 1998.

[80] I. Bardi, R. Remski, D. Perry, Z. Cendes, "Plane wave Scattering from Frequency Selective Surfaces by the Finite-Element Method", IEEE Trans. Magnetics, Vol. 38, No. 2, pp. 641-644, Mar. 2002.

[81] P. Harms, R. Mittra, W. Ko, "Implementation of the periodic boundary condition in the Finite-difference time-domain algorithm for FSS structures", IEEE Trans. Antennas Propag., Vol 42, No. 9, pp. 1317-1324, Sept. 1994.

[82] G. Zheng, A. A. Kishk, A. W. W. Glisson, A. B. Yakovlev, "Implementation of Mur's absorbing boundaries with periodic structures to speed up the design process using finitedifference time-domain method", Progress in Electromagnetics Research, PIER 58, pp. 101114, 2006. 
[83] W. Yu, R. Mittra, "A conformal FDTD software package modelling antennas and microstrip circuit components" IEEE Antennas Propag. Magazine, Vol. 45, issue 5, pp. 2839 , Oct. 2000.

[84] T. Su, Y. Liu, W. Yu, R. Mittra, "A Conformal Mesh-Generating Technique for the Conformal Finite-Difference Time-Domain (CFDTD) Method", IEEE Antennas Propag. Magazine, Vol. 46, No. 1, pp.37-49, Feb. 2004.

[85] A. Qing, "Vector Spectral-Domain Method for the analysis of Frequency Selective Surfaces", Progress in electromagnetics Research, PIER 65, pp. 201-232, 2006.

[86] D. B. Davidson, J. T. Aberie, "An introduction to spectral domain method of moments formulation", IEEE Antennas Propag. Magazine, Vol. 46, No. 3, pp. 11-19, June 2004.

[87] L. Gurel, W. C. Chew, "Recursive T-matrix algorithms for the solution of electromagnetic scattering from strip and patch geometries", IEEE Trans. Antennas Propag., Vol. 41, No. 1, pp. 91-99, 1993.

[88] A. Sahin, E. L. Miller, "Recursive T-Matrix Methods for Scattering from Multiple Dielectric and Metallic Objects", IEEE Trans. Antennas Propag., Vol. 46, No. 5, pp. 672-678, May 1998.

[89] R. J. Langley, E. A. Parker, "Equivalent circuit model for arrays of square loops", Electronic Letters, Vol. 18, No. 7, pp. 294-296, 1982.

[90] N. Marcuvitz, "Waveguide Handbook", Dover Publications Inc., pp. 280-285, New York 1965.

[91] C. H. H. Sung, K. W. Sowerby, A. G. Williamson, "Equivalent Circuit Modelling of a Frequency Selective Plasterboard Wall", IEEE Antennas Propag. Int. Symposium, Vol. 4A, pp. 400-403, July 2005 .

[92] K. S. Yee, "Numerical Solution of Initial Boundary Value Problems involving Maxwell's Equations in Isotropic Media", IEEE Trans. Antennas Propag., Vol. 14. No.3, pp. 302-307, May 1966.

[93] G. Mur, "Absorbing Boundary Conditions for the finite-difference approximation of the time-domain electromagnetic-field equations", IEEE Trans. Electromagn. Compat., Vol. EMC-23, No.4, pp. 377-382, 1981.

[94] M. Raspopoulos, S. Stavrou, "Frequency Selective Surfaces on Building Materials - Air gap Impact", IET Electronic Letters, Vol. 43, Issue 13, pp. 700-702, June 2007.

[95] S. H. Hoi, J. K. Park, S. K. Kim, J. Y. Park, "A new Ultra-wideband antenna for UWB applications", Microw. and Opt. Tech. Letters, Vol. 40, No. 5, pp 399-401, March 2004. 
[96] G. H. H. Sung, K. W. Sowerby, A. G. Williamson, "The impact of Frequency Selective Surfaces applied to standard wall construction materials", IEEE International Symposium on Antennas and Propagation 2004, vol. 2, pp. 2187-2190, 20-25 June 2004.

[97] A. H. Muquibel, A. S. Jazi, "A new formulation for Characterisation of Materials Based on Insertion Transfer Function", IEEE Trans. Microw. Theory and Techniques, Vol. 51, No. 8, August 2003.

[98] M. Raspopoulos, S. Stavrou, "Ray Tracing of Indoor Frequency Selective Environments", Submitted to IEEE Antennas Propag. Letters. Under Review.

[99] C. W. Trueman, R. Paknys, J.Zhao, D. Davis, B. Segal, "Ray Tracing Algorithm for Indoor Propagation", Applied Computational Electromagnetics Society. Proc 16th Annual Review of Progress in Applied Computational Electromagnetics, Monterey, CA., March 2000, pp. 493-500.

[100] Vera B. Anand, "Computer Graphics and Geometric Modelling for Engineers", John Wiley \& Sons, 1993.

[101] F. A. Chaudhry, M. Raspopoulos, S. Stavrou, "Effect of Frequency Selective Surfaces on radio wave propagation in indoor environments", $11^{\text {th }}$ European Wireless Conf. 2005, Nicosia, Cyprus, Vol. 2, pp. 732-736, April 2005.

[102] M. Raspopoulos, F. A. Chaudhry, S. Stavrou, "Radio Propagation in Frequency Selective Buildings", Euro. Trans. Telecoms, Vol. 17, pp. 407-413, March 2006.

[103] M. Raspopoulos, S. Stavrou, "Frequency Selective Buildings through Frequency Selective Surfaces", Submitted to IEEE Trans. Antennas Propag. Under Review.

[104] J. H. Winters, "On the capacity of Radio Communication Systems with Diversity in Rayleigh Fading Environment", IEEE Journal on Selected Areas in Commun., Vol. SAC-5, No. 5, pp. 871-878, June 1987.

[105] G. J. Foschini, M. J. Gans, "On Limits of Wireless Communications in a Fading Environment when using Multiple Antennas", Wireless Personal Communications, Vol. 6, pp 311-335, 1998.

[106] Persefoni Kyritsi, Donald C. Cox, Reinaldo Valenzuela, Peter W. Wolniasky, "Correlation Analysis Based on MIMO Channel Measurements in an Indoor Environment", IEEE Journal on selected areas in communications, Vol. 21, No. 5, June 2003.

[107] Zhongwei Tang, Mohan, A.S., "Evaluation of the performance of indoor MIMO systems", Antennas, Propagation and EM Theory, 2003. Proceedings. 2003 6th International Symposium on 28 Oct.-1 Nov. 2003 pp. $553-555$ 
[108] E. Telatar, "Capacity of multi-antenna Gaussian channels", Eur. Trans. Telecommun., vol.10, no. 6, pp. 585-595, Nov/Dec 1999.

[109] J.B. Andersen, "Array gain and capacity for known random channels with multiple element arrays at both ends", IEEE J. Selected Areas Commun., Vol 18, pp 2172-2178, Nov. 2000.

[110] Zhengqing Yun, Magdy F. Iskander, Zhijun Zhang, "Complex-Wall Effect on Propagation Characteristics and MIMO Capacities for an Indoor Wireless Communication Channel", IEEE Trans. Antennas and Propag., Vol. 52, No. 4, April 2004.

[111] Antonio Forenza, Robert W. Heath Jr., "Impact of Antenna Geometry on MIMO Communication in Indoor Clustered Channels", IEEE Antennas and Propag. Society Symposium, 2004. Vol. 2, 20-25 June 2004 pp. 1700 - 1703

[112] D. Gesbert, M. Shafi, D. S. Shiu, P. J. Smith, A. Naguib, "From Theory to Practice: An overview of MIMO Space-Time Coded Wireless Systems", IEEE Journal on Selected Areas in Commun., Vol. 21. No. 3, pp. 281-302, April 2003.

[113] A. Doufexi, E. Tameh, A. Nix, A. Pal, M. Beach, C. Williams, "Throughput and Coverage of WLANs Employing STBC under Different Channel Conditions", Ist Internat. Symp. On Wireless Comm. Systems 2004, pp 367-372, 20-22 Sept 2004.

[114] E. Akay and E. Ayanoglu, "High Performance Viterbi Decoder for OFDM Systems", $59^{\text {th }}$ IEEE Vehic. Techn. Conf. 2004, VTC-2004, Vol. 1, pp. 323-327, May 2004.

[115] N. Monk, H.S. Winbigler, "Communication with moving trains in tunnels", IRE Trans. On Veh. Comm., vol. VC-7, pp. 21-8, Dec. 1956.

[116] D. J. R. Martin, "Leaky-Feeder Radio Communication: A Historical Review", $34^{\text {th }}$ IEEE Veh. Comm. Conf., vol. 34, pp. 25-30, 21-23 May 1984.

[117] Q. V. Davis, D. J. R. Martin, R. W. Haining, "Microwave Radio in Mines and Tunnels", 34 $4^{\text {th }}$ IEEE Veh. Comm. Conf., vol. 1, pp. 375-382, 1-3 May 1989.

[118] K. J. Bye, "Leaky-Feeders for cordless communication in the office", $8^{\text {th }}$ European Conf. on Electronics, E UROCON 88, pp.387-390, 13-17 June 1988.

[119] Y. P. Zhang, "Indoor Radiated-Mode Leaky Feeder Propagation at $2.0 \mathrm{GHz}$ ", IEEE Trans. Veh. Tech., vol.50, No.2, March 2001.

[120] J.P. Daniel, "Mutual coupling between antennas for emission or reception application to passive and active dipoles", IEEE Trans. On Antennas and Propagation, vol. 22, Issue 2, pp.347-349, 1984. 